Results of the U.S. Geological Survey's Analytical

Evaluation Program for Standard Reference Samples

Distributed in March 2002

By Mark T. Woodworth and Brooke F. Connor

U.S. GEOLOGICAL SURVEY

Open-File Report 02-243

Lakewood, Colorado

2002 


\section{DEPARTMENT OF THE INTERIOR}

Gayle A. Norton, Secretary

U.S. GEOLOGICAL SURVEY

Charles G. Groat, Director

For additional information write to:

Chief, Branch of Quality Systems

U.S. Geological Survey

Water Resources Division

Box 25046, Mail Stop 401

Denver Federal Center

Denver, Colorado 80225-0046
Copies of this report can be purchased from:

U.S. Geological Survey Branch of Information Services DFC, Bldg. 810, Box 25286

Denver, Colorado 80225-0286 888-ASK-USGS 


\section{CONTENTS}

Page

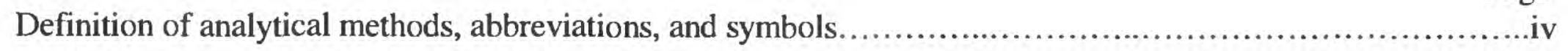

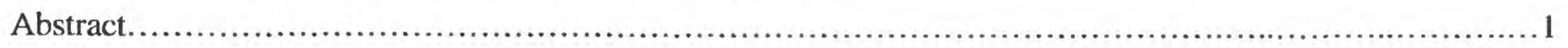

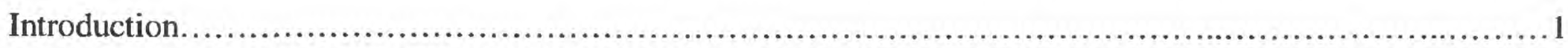

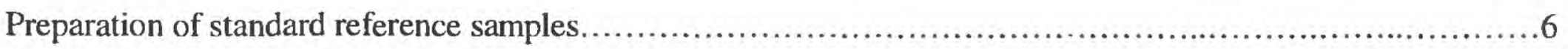

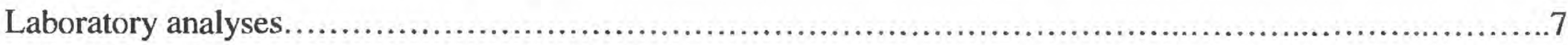

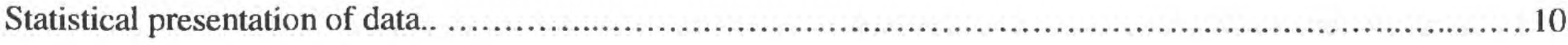

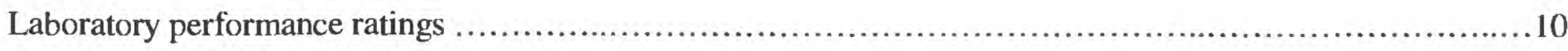

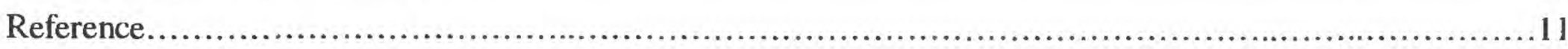

FIGURE

Figure 1. Statistical parameters shown on data graphs in tables 11-16.

\section{TABLES}

1. USGS used laboratories that participated in the analyses of standard reference samples distributed in March 2002 .

2. Other laboratory participants in the analyses of standard reference samples distributed in March 2002 ............4

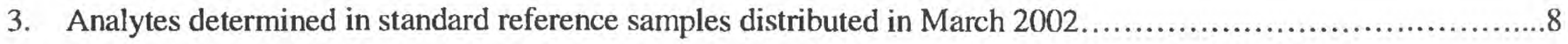

4. Overall laboratory performance ratings for standard reference samples distributed in March $2002 \ldots \ldots \ldots \ldots \ldots . .12$

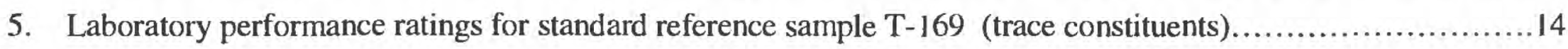

6. Laboratory performance ratings for standard reference sample M-162 (major constituents) $\ldots \ldots \ldots \ldots \ldots \ldots \ldots . . . . . . .26$

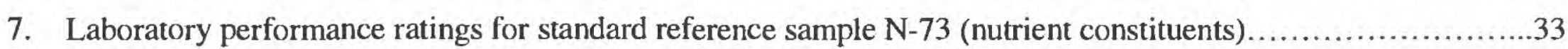

8. Laboratory performance ratings for standard reference sample $\mathrm{N}-74$ (nutrient constituents) .....................35

9. Laboratory performance ratings for standard reference sample P-38 (low ionic-strength constituents).............37

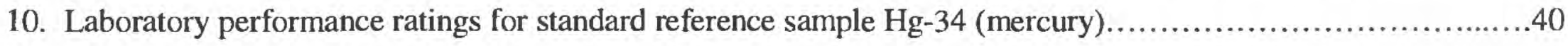

11. Statistical summary of reported data for standard reference sample T-169 (trace constituents)..................

12. Statistical summary of reported data for standard reference sample M-162 (major constituents) .................69

13. Statistical summary of reported data for standard reference sample $\mathrm{N}-73$ (nutrient constituents) .................85

14. Statistical summary of reported data for standard reference sample N-74 (nutrient constituents)..................90

15. Statistical summary of reported data for standard reference sample P-38 (low ionic-strength constituents).........95

16. Statistical summary of reported data for standard reference sample $\mathrm{Hg}-34$ (mercury).......................... 106

17. Most probable values for constituents and properties in standard reference samples distributed in March $2002 . .107$ 


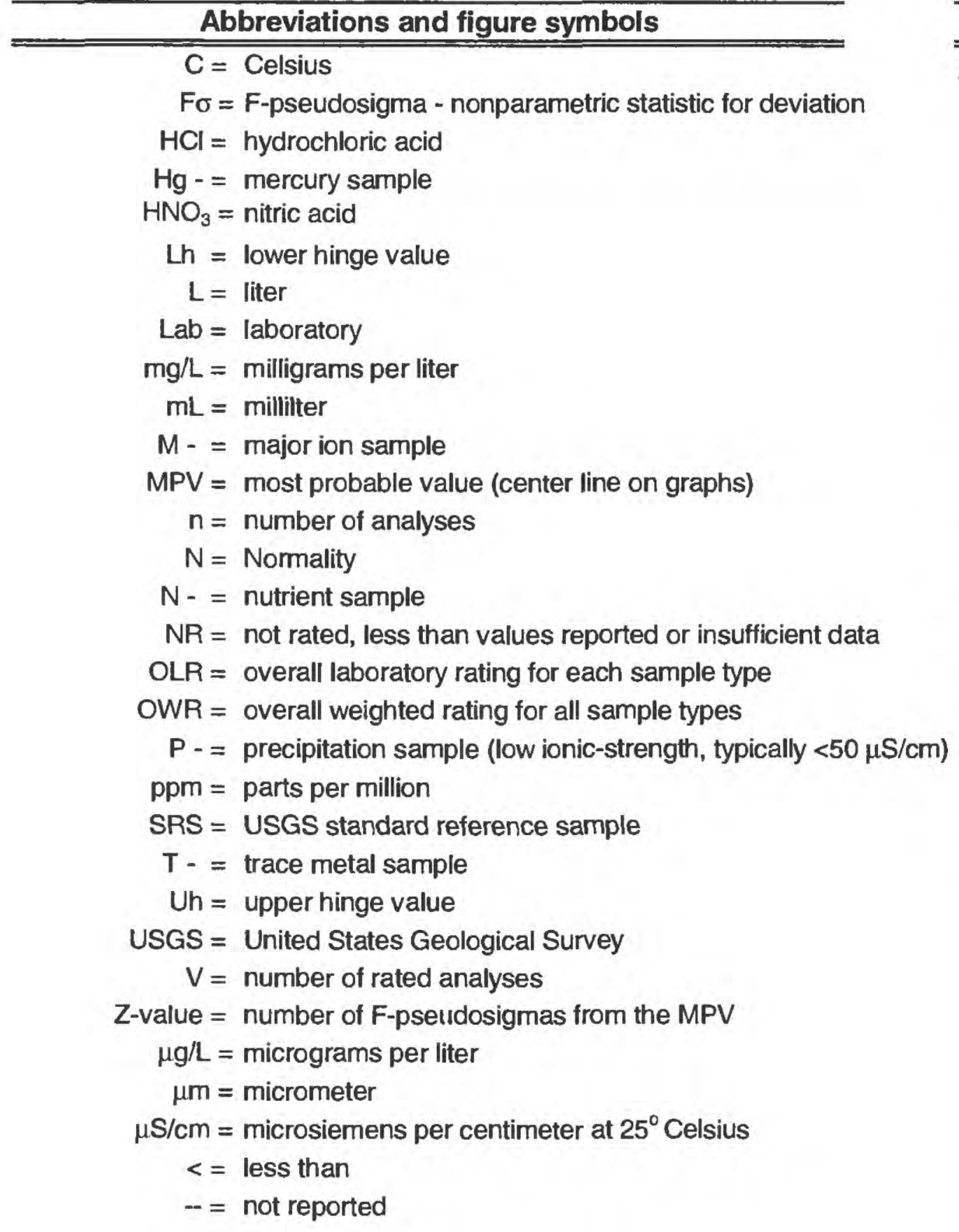

\section{Formulas}

MPV $=$ median value (excluding less than values)

F-pseudosigma $(\mathrm{Fo})=(\mathrm{Uh}-\mathrm{U}) / 1.349$

$\mathrm{Uh}=$ median of the upper half of the reported values (excluding less than values)

$\mathrm{L}=$ median of the lower half of the reported values (excluding less than values)

Z-value $=($ reported value - MPV)/F-pseudosigma

$O L R=$ mean of all rated analytes for sample type

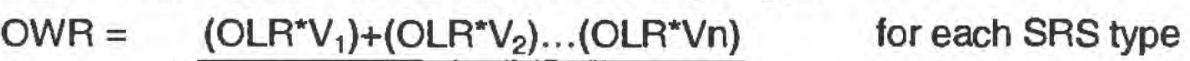

$\left(V_{1}+V_{2}+\ldots . . . V n\right)$

\begin{tabular}{ll}
\hline \multicolumn{2}{c}{ Ratings } \\
\hline \hline Rating & Absolute Z-value \\
\cline { 2 - 2 } 3 (Excellent) & 0.00 to 0.50 \\
2 (Good) & 0.51 to 1.00 \\
1 (Matisfactory) & 1.01 to 1.50 \\
0 (Unsatisfactory) & 1.51 to 2.00 \\
& Greater than 2.00
\end{tabular}

\begin{aligned} \hline & Analytical methods and codes \\ \hline \hline Code & Method \\ 1 & Other \\ 2 & Atomic absorption: direct, air \\ 3 & Atomic absorption: graphite furnace \\ 4 & Inductively coupled plasma \\ 5 & Direct current plasma \\ 6 & Inductively coupled plasma/mass spectrometry \\ 7 & Ion chromatography \\ 8 & Atomic absorption: cold vapor \\ 9 & Atomic fluorescence \\ 10 & Atomic absorption: extraction \\ 11 & Atomic absorption: hydride \\ 12 & Flame emission \\ 20 & Titration: colorimetric \\ 21 & Titration: electrometric \\ 22 & Colorimetric \\ 40 & Ion selective electrode \\ 41 & Electrometric [pH and specific conductance] \\ 50 & Gravimetric \\ 51 & Turbidimetric \end{aligned}

Analytical methods and codes

Atomic absorption: direct, air

Inductively coupled plasma

Inductively coupled plasma/mass spectrometry

Ion chromatography

Atomic absorption: cold vapor

Atomic absorption: extraction

dric

Titration: colorimetric

Titration: electrometric

Colorimetric

Ion selective electrode

Gravimetric 


\title{
RESULTS OF THE U.S. GEOLOGICAL SURVEY'S ANALYTICAL EVALUATION \\ PROGRAM FOR STANDARD REFERENCE SAMPLES DISTRIBUTED IN MARCH 2002
}

\author{
By Mark T. Woodworth and Brooke F. Connor
}

\begin{abstract}
This report presents the results of the U.S. Geological Survey's analytical evaluation program for six standard reference samples -- T-169 (trace constituents), M-162 (major constituents), N-73 (nutrient constituents), N-74 (nutrient constituents), P-38 (low ionic-strength constituents), and Hg-34 (mercury) -- that were distributed in March 2002 to laboratories enrolled in the U.S. Geological Survey sponsored interlaboratory testing program. Analytical data received from 93 laboratories were evaluated with respect to overall laboratory performance and relative laboratory performance for each analyte in the six reference samples. Results of these evaluations are presented in tabular form. Also presented are tables and graphs summarizing the analytical data provided by each laboratory for each analyte in the six standard reference samples. The most probable value for each analyte was determined using nonparametric statistics.
\end{abstract}

\section{INTRODUCTION}

The U.S. Geological Survey (USGS) conducts an interlaboratory analytical evaluation program semiannually. This program provides a variety of standard reference samples (SRSs) to accomplish quality assurance testing of laboratories and to provide an adequate supply of samples that contribute to quality control programs of participating laboratories. Natural-matrix reference materials are preferred for use in this interlaboratory evaluation program. A series of samples are prepared and distributed each spring and fall. The program began in 1962 with a single sample containing major constituents that was prepared from distilled water and reagent-grade chemicals. Twenty-three USGS laboratories participated in the first analytical evaluation program. Since that time, objectives of the program have been to:

(1) evaluate and improve the performance of USGS and other participating laboratories;

(2) provide a library of carefully prepared, homogeneous, stable, reference materials for use in the quality control programs of laboratories;

(3) identify analytical problem areas;

(4) identify quality assurance needs with respect to environmental analyses and develop new reference materials to meet these needs; and

(5) evaluate the accuracy and precision of analytical methods.

A total of 274 USGS and non-USGS laboratories are enrolled in the program, which can currently provide 5 different types of SRSs:

1. Trace constituents.

2. Major constituents.

3. Nutrient constituents.

4. Low ionic-strength constituents.

5. Mercury. 
Though this is not a laboratory certification program, participation in this continuing quality assurance program is mandatory for all laboratories providing water-quality data for USGS sponsored reports or storage in the USGS national databases. The results from this study can be used to alert participating laboratories of possible deficiencies in their analytical operations and provide reference materials for laboratory quality-control programs. Laboratories that provide data for the USGS are identified with a laboratory identification number while all other laboratories are kept confidential.

A supply of SRSs from previous evaluations, is available. USGS offices and participating laboratories can purchase these SRSs for further testing, continuing quality assurance, and qualitycontrol programs by contacting:

U.S. Geological Survey

Branch of Quality Systems

SRS Purchasing

Denver Federal Center, Bldg. 53

P. O. Box 25046, MS 401

Denver, Colorado 80225-0046

(303) 236-1875

This report summarizes the analytical results submitted by 93 laboratories for the March 2002 evaluation (table 1 and table 2). Analytical results for the following are presented in this report:

$\begin{array}{llll}\text { T-169 } & \text { Trace constituents } & \text { N-74 } & \text { Nutrient constituents } \\ \text { M-162 } & \text { Major constituents } & \text { P-38 } & \text { Low ionic-strength constituents } \\ \text { N-73 } & \text { Nutrient constituents } & \text { Hg-34 } & \text { Mercury }\end{array}$

Laboratories that are providing analytical services to USGS offices are requested to analyze the appropriate SRSs for the same analytes requested by the USGS offices. All laboratories are requested to include the analytical methods used to determine the concentration of each analyte. When analytical method information was provided, it has been included in tables 11-16.

Not all SRSs are requested or necessarily analyzed by all the laboratories; nor do all laboratories enrolled in the program participate in each evaluation. 
Table 1. USGS used laboratories that participated in the analyses of standard reference samples distributed in March 2002

\begin{tabular}{|c|c|c|c|}
\hline Lab & Participating Laboratory & City & State \\
\hline 1 & U.S. Geological Survey - National Water Quality Laboratory & Denver & $\mathrm{CO}$ \\
\hline 4 & U.S. Geological Survey - Utah District Laboratory & Salt Lake City & UT \\
\hline 7 & U.S. Geological Survey Geologic Division - Coal Analytical Laboratory & Denver & $\mathrm{CO}$ \\
\hline 12 & Metro Wastewater Reclamation District & Denver & $\mathrm{CO}$ \\
\hline 16 & Oklahoma Department of Environmental Quality & Oklahoma City & $\mathrm{OK}$ \\
\hline 21 & UC Davis - Department of Environmental Science \& Policy & Davis & $\mathrm{CA}$ \\
\hline 23 & City of Fort Collins Water Quality Laboratory & Ft. Collins & $\mathrm{CO}$ \\
\hline 25 & Kentucky Geological Survey & Lexington & $\mathrm{KY}$ \\
\hline 33 & U.S. Geological Survey - Georgia District Laboratory & Atlanta & GA \\
\hline 46 & Wisconsin State Laboratory of Hygiene & Madison & WI \\
\hline 59 & Division of Consolidated Laboratory Services & Richmond & VA \\
\hline 70 & University of Iowa Hygienic Laboratory & Des Moines & IA \\
\hline 72 & New Jersey Department of Health Laboratory & Trenton & NJ \\
\hline 89 & Monroe County Environmental Health Laboratory & Rochester & NY \\
\hline 102 & Heidelberg College & Tiffin & $\mathrm{OH}$ \\
\hline 109 & North Dakota State Water Commission Laboratory & Bismarck & ND \\
\hline 110 & U.S. Geological Survey - New York District Laboratory & Troy & NY \\
\hline 118 & Virginia Tech - Occoquan Watershed Monitoring Laboratory & Manassas & VA \\
\hline 134 & Ocala Water Quality and Research Laboratory & Ocala & $\mathrm{FL}$ \\
\hline 142 & North Dakota Department of Health & Bismarck & ND \\
\hline 147 & U.S. Geological Survey - Surface Water Quality Research & Boulder & $\mathrm{CO}$ \\
\hline 180 & Clean Water Services (formally Unified Sewerage Agency) & Hillsboro & OR \\
\hline 193 & Vermont Department of Enviromental Conservation Laboratory & Waterbury & VT \\
\hline 198 & Maryland Department of Health and Mental Hygiene & Baltimore & MD \\
\hline 205 & Olsen Agriculture Laboratory & McCook & $\mathrm{NE}$ \\
\hline 208 & U.S. Geological Survey - WRD San Diego & San Diego & $\mathrm{CA}$ \\
\hline 212 & Severn Trent Laboratory & Arvada & $\mathrm{CO}$ \\
\hline 224 & University of Arkansas - Water Quality Laboratory & Fayetteville & $\mathrm{AR}$ \\
\hline 234 & City of Wichita Laboratory & Wichita & KS \\
\hline 255 & Colorado Springs Utilities - Water Resource Department & Colorado Springs & $\mathrm{CO}$ \\
\hline 315 & Wisconsin District WEBB Laboratory & Middleton & WI \\
\hline 319 & Fairfax County Environmental Services & Lorton & VA \\
\hline 330 & Kennecott Environmental Laboratory & Magna & UT \\
\hline 333 & U.S. Geological Survey - WEBB Colorado District Office & Lakewood & $\mathrm{CO}$ \\
\hline 341 & Michigan Department of Environmental Quality & Lansing & MI \\
\hline 356 & Washington State Department of Ecology - Manchester Laboratory & Port Orchard & WA \\
\hline 366 & TriMatrix Laboratory & Grand Rapids & MI \\
\hline 370 & Guardian Systems, Inc. & Leeds & AL \\
\hline 372 & Alabama Power Company & Calera & AL \\
\hline 373 & City of Tulsa - Quality Assurance Laboratory & Tulsa & $\mathrm{OK}$ \\
\hline 374 & U.S. Geological Survey - Miami Subdistrict Laboratory & Miami & FL \\
\hline 375 & U.S. Geological Survey - Upper Midwest Environmental Science Center & La Crosse & WI \\
\hline
\end{tabular}


Table 2. Other laboratory participants in the analyses of standard reference samples distributed in March 2002

Participating Laboratory

City

State

Albion Environmental

College Station TX

Aqua Tech Environmental Laboratory (ATEL)

Marion

$\mathrm{OH}$

Boise City Water Quality Laboratory

Boise

ID

City of Northglenn - Water Treatment Facility

Northglenn

$\mathrm{CO}$

City of Tallahassee -Water Quality Laboratory

Tallahassee

FL

Columbia Analytical Services

Rochester

NY

Darrin Freshwater Institute

Denver Water Department

Bolton Landing NY

Desert Research Institute

Environmental Task Force Laboratory

Florida Department of Environmental Protection

Denver

$\mathrm{CO}$

Reno NV

Frontier Geosciences Inc.

High Sierra Water Laboratory

Huffman Laboratories

Institute of Ecosystem Studies

Kansas Geological Survey

Lower Colorado River Authority - Environmental Laboratory Services

Madison Public Health Laboratory

Stevens Point WI

Tallahassee FL

Seattle WA

Truckee CA

Golden $\quad \mathrm{CO}$

Millbrook NY

Lawrence KS

Austin TX

Madison WI

Montana Bureau of Mines \& Geology

Old Dominion University - Applied Marine Research Laboratory

Ouachita Baptist University - Department of Biology

Pennsylvania Department of Environmental Protection

Rocky Mount Waste Water Treatment Plant

Severn Trent Laboratory

South Florida Water Management District

Southwest Florida Water Management District

Suffolk County Water Authority

U.S. Bureau of Reclamation

U.S. Bureau of Reclamation

U.S. Bureau of Reclamation

U.S. Bureau of Reclamation

U.S. Bureau of Reclamation

U.S. Department of Agriculture - Cooperative Chemical Analytical Laboratory

MT

Norfolk VA

Arkadelphia AR

Harrisburg PA

Rocky Mount NC

Tallahassee FL

West Palm Beach FL

Brooksville FL

Hauppauge NY

Denver $\mathrm{CO}$

Boise ID

Alamosa $\mathrm{CO}$

Boulder City NV

Bismarck ND

Corvallis OR

Ft. Collins $\mathrm{CO}$

Edison NJ

Athens GA

Honolulu HI

Solomons MD

Cambridge MD

Santa Fe Springs CA

Champaign IL

Laramie WY 
Table 2. Other laboratory participants in the analyses of standard reference samples distributed in March 2002 -- continued

Middle East Participating Laboratory

Location

Geological Survey of Israel Laboratory

Jerusalem

Mekorot Laboratory

Eylat

Israel

Mekorot Laboratory

Ashqelon

Israel

Mekorot Laboratory - Rosh-Haayn Laboratory

Ramla

Israel

Mekorot Water Co. Ltd. - Central Lab

Nazaret Illit

Israel

Public Health Laboratory

Public Health Laboratory - Ministry of Health

Tel Aviv

Israel

Public Health Laboratory - Sabha Medical Clinic

Beer Sheva

Water Authority of Jordan

Gaza

Israel

Amman

Israel

via West Bank

Jordan 


\section{PREPARATION OF STANDARD REFERENCE SAMPLES}

All of the SRSs used in this evaluation were prepared by USGS personnel located in Lakewood, Colorado, and were analyzed for analyte concentrations and physical property values before mailing. A supply of these SRSs is maintained and are available to purchase by participating laboratories and USGS offices for use in their quality-control programs.

Trace constituents sample T-169 was prepared using water collected from the South Platte west of Bailey, Colorado. The water was pumped through a 0.2- and 0.1-micrometer $(\mu \mathrm{m})$ filter into a 1325-liter (L) polypropylene drum. The water was continuously circulated and passed through a $0.1-\mu \mathrm{m}$ filter and ultraviolet sterilizer for 24 hours. The water was then acidified to a $\mathrm{pH}<2$ with nitric acid $\left(\mathrm{HNO}_{3}\right)$ and chlorinated to 5 parts per million ( $\left.\mathrm{ppm}\right)$ free chlorine with sodium hypochlorite. The trace constituent concentrations were adjusted by adding reagent-grade chemicals. The sample was circulated through a $0.1-\mu \mathrm{m}$ filter and an ultraviolet sterilizer for an additional 24 hours prior and during bottling. The 500-milliliter $(\mathrm{mL})$ polypropylene bottles and caps were acid leached with $0.16 \mathrm{~N} \mathrm{HNO}_{3}$, deionized-water rinsed, and autoclave sterilized.

Major constituents sample M-162 was prepared using water collected from Bear Creek east of Kitridge, Colorado. The water was pumped through a $0.2-$ and $0.1-\mu \mathrm{m}$ filter into a $1325-\mathrm{L}$ polypropylene drum. The water was continuously circulated and passed through a $0.1-\mu \mathrm{m}$ filter and ultraviolet sterilizer for 24 hours. The water was then chlorinated to 5-ppm free chlorine with sodium hypochlorite. The major constituent concentrations were adjusted by adding reagent-grade chemicals. The sample was circulated an additional 24 hours, then allowed to sit for 48 hours. During bottling, the sample was pumped through an ultraviolet sterilizer and a $0.1-\mu \mathrm{m}$ filter. The $500-\mathrm{mL}$ polypropylene bottles and caps were acid leached with $0.16 \mathrm{~N}$ $\mathrm{HNO}_{3}$, deionized-water rinsed, and autoclave sterilized.

Nutrient constituents sample $\mathrm{N}-73$ was prepared in a 50-L polypropylene carboy using deionized water. This SRS was prepared the week prior to sample distribution. The water was circulated through a $0.1-\mu \mathrm{m}$ filter and kept chilled with ice ( 12 degrees Celsius) during the entire preparation procedure. Ultraviolet sterilization was performed up until the addition of reagent-grade chemicals. The $60-\mathrm{mL}$ amber glass vials and teflon-faced rubber-lined caps were acid leached with $0.1 \mathrm{~N}$ hydrochloric acid $(\mathrm{HCl})$, deionized-water rinsed, and autoclave sterilized.

Nutrient constituents sample N-74 was prepared in a 190-L polypropylene drum using water collected from Clear Creek east of Blackhawk, Colorado. This SRS was prepared the week prior to sample distribution. The water was circulated through a $0.1-\mu \mathrm{m}$ filter and kept chilled with ice (12 degrees Celsius) during the entire preparation procedure. Ultraviolet sterilization was performed up until the addition of reagent-grade chemicals. The $250-\mathrm{mL}$ polyethylene bottles were acid leached with $0.1 \mathrm{~N} \mathrm{HCl}$, deionized-water rinsed, and autoclave sterilized.

Low ionic-strength constituents sample P-38 was prepared in a $600-\mathrm{L}$ polypropylene drum with snow collected west of Idledale, Colorado. The desired phosphate concentration was obtained by adding a reagent-grade chemical. Prior and during bottling, the sample was circulated through a $0.1-\mu \mathrm{m}$ filter and an ultraviolet sterilizer. The $500-\mathrm{mL}$ polypropylene bottles and caps were acid leached with $0.16 \mathrm{~N} \mathrm{HNO}_{3}$, deionized-water rinsed, and autoclave sterilized. 
Mercury sample Hg-34 was prepared using deionized water that was filtered by a Nanopure system. The sample was prepared in a $45-\mathrm{L}$ glass carboy. It was preserved with $5 \mathrm{~mL} / \mathrm{L} 12 \mathrm{~N}$ $\mathrm{HCl}$. The desired mercury concentration was obtained by adding a mercury standard solution. The 250-mL borsilicate glass bottles and teflon-lined caps were new, acid leached, and deionized-water rinsed.

\section{LABORATORY ANALYSES}

The participating laboratories were asked to determine constituents that are summarized in table 3 . The number of analytes range from 28 in T-169 (trace constituents) to 1 in $\mathrm{Hg}-34$ (mercury). 
Table 3. Analytes determined in standard reference samples distributed in March 2002

[mg/L, milligrams per liter; $\mu \mathrm{g} / \mathrm{L}$, micrograms per liter; $\mu \mathrm{S} / \mathrm{cm}$, microsiemens per centimeter at 25 degrees Celsius]

\begin{tabular}{|c|c|c|c|c|c|c|c|c|}
\hline \multicolumn{2}{|c|}{ Constituent or Property } & \multirow{2}{*}{$\frac{\text { Units }}{\mathrm{mg} / \mathrm{L}}$} & \multirow[t]{2}{*}{ T-169 } & \multirow[t]{2}{*}{ M-162 } & \multirow[t]{2}{*}{$\mathrm{N}-73$} & \multirow[t]{2}{*}{$\mathrm{N}-74$} & \multirow{2}{*}{$\frac{\text { P-38 }}{X}$} & \multirow[t]{2}{*}{ Hg-34 } \\
\hline Acidity & Acidity as $\mathrm{CaCO}_{3}$ & & & & & & & \\
\hline Alk & Alkalinity as $\mathrm{CaCO}_{3}$ & $\mathrm{mg} / \mathrm{L}$ & & $\mathrm{X}$ & & & & \\
\hline $\mathrm{Ag}$ & Silver & $\mu \mathrm{g} / \mathrm{L}$ & $\mathrm{X}$ & & & & & \\
\hline $\mathrm{Al}$ & Aluminum & $\mu \mathrm{g} / \mathrm{L}$ & $X$ & & & & & \\
\hline As & Arsenic & $\mu \mathrm{g} / \mathrm{L}$ & $\mathrm{X}$ & & & & & \\
\hline B & Boron & $\mu \mathrm{g} / \mathrm{L}$ & $\mathrm{X}$ & $\mathrm{X}$ & & & & \\
\hline $\mathrm{Ba}$ & Barium & $\mu \mathrm{g} / \mathrm{L}$ & $\mathrm{X}$ & & & & & \\
\hline $\mathrm{Be}$ & Beryllium & $\mu \mathrm{g} / \mathrm{L}$ & $\mathrm{X}$ & & & & & \\
\hline $\mathrm{Ca}$ & Calcium & $\mathrm{mg} / \mathrm{L}$ & $\mathrm{X}$ & $\mathrm{X}$ & & & $\mathrm{X}$ & \\
\hline $\mathrm{Cd}$ & Cadmium & $\mu \mathrm{g} / \mathrm{L}$ & $\mathrm{X}$ & & & & & \\
\hline $\mathrm{Cl}$ & Chloride & $\mathrm{mg} / \mathrm{L}$ & & $\mathrm{X}$ & & & $\mathrm{X}$ & \\
\hline $\mathrm{Co}$ & Cobalt & $\mu \mathrm{g} / \mathrm{L}$ & $\mathrm{X}$ & & & & & \\
\hline $\mathrm{Cr}$ & Chromium & $\mu \mathrm{g} / \mathrm{L}$ & $\mathrm{X}$ & & & & & \\
\hline $\mathrm{Cu}$ & Copper & $\mu \mathrm{g} / \mathrm{L}$ & $\mathrm{X}$ & & & & & \\
\hline ROE & Dissolved Solids & $\mathrm{mg} / \mathrm{L}$ & & $\mathrm{X}$ & & & & \\
\hline $\mathrm{F}$ & Fluoride & $\mathrm{mg} / \mathrm{L}$ & & $X$ & & & $\mathrm{X}$ & \\
\hline $\mathrm{Fe}$ & Iron & $\mu \mathrm{g} / \mathrm{L}$ & $\mathrm{X}$ & & & & & \\
\hline $\mathrm{Hg}$ & Mercury & $\mu \mathrm{g} / \mathrm{L}$ & & & & & & $\mathrm{X}$ \\
\hline $\mathrm{K}$ & Potassium & $\mathrm{mg} / \mathrm{L}$ & $\mathrm{X}$ & $\mathrm{X}$ & & & $\mathrm{X}$ & \\
\hline$\underline{\mathrm{Li}}$ & Lithium & $\mu \mathrm{g} / \mathrm{L}$ & $\mathrm{X}$ & & & & & \\
\hline $\mathrm{Mg}$ & Magnesium & $\mathrm{mg} / \mathrm{L}$ & $\mathrm{X}$ & $\mathrm{X}$ & & & $\mathrm{X}$ & \\
\hline $\mathrm{Mn}$ & Manganese & $\mu \mathrm{g} / \mathrm{L}$ & $\mathrm{X}$ & & & & & \\
\hline Mo & Molybdenum & $\mu \mathrm{g} / \mathrm{L}$ & $\mathrm{X}$ & & & & & \\
\hline $\mathrm{Na}$ & Sodium & $\mathrm{mg} / \mathrm{L}$ & $X$ & $\mathrm{X}$ & & & $\mathrm{X}$ & \\
\hline $\mathrm{NH}_{3}$ as $\mathrm{N}$ & Ammonia & $\mathrm{mg} / \mathrm{L}$ & & & $\mathrm{X}$ & $\mathrm{X}$ & & \\
\hline $\mathrm{NH}_{3}+\operatorname{Org} \mathrm{N}$ as $\mathrm{N}$ & Ammonia + Organic $\mathrm{N}$ & $\mathrm{mg} / \mathrm{L}$ & & & $\mathrm{X}$ & $\mathrm{X}$ & & \\
\hline $\mathrm{Ni}$ & Nickel & $\mu \mathrm{g} / \mathrm{L}$ & $\mathrm{X}$ & & & & & \\
\hline $\mathrm{NO}_{3}$ as $\mathrm{N}$ & Nitrate & $\mathrm{mg} / \mathrm{L}$ & & & $\mathrm{X}$ & $\mathrm{X}$ & & \\
\hline $\mathrm{Pb}$ & Lead & $\mu \mathrm{g} / \mathrm{L}$ & $X$ & & & & & \\
\hline $\mathrm{pH}$ & $\mathrm{pH}$ & unit & & $\mathrm{X}$ & & & $\mathrm{X}$ & \\
\hline $\mathrm{PO}_{4}$ as $\mathrm{P}$ & Orthophosphate & $\mathrm{mg} / \mathrm{L}$ & & & $X$ & $\mathrm{X}$ & $\mathrm{X}$ & \\
\hline total $P$ as $P$ & Phosphorus & $\mathrm{mg} / \mathrm{L}$ & & $X$ & $X$ & $X$ & & \\
\hline $\mathrm{Sb}$ & Antimony & $\mu \mathrm{g} / \mathrm{L}$ & $\mathrm{X}$ & & & & & \\
\hline $\mathrm{Se}$ & Selenium & $\mu \mathrm{g} / \mathrm{L}$ & $\mathrm{X}$ & & & & & \\
\hline $\mathrm{SiO}_{2}$ & Silica & $\mathrm{mg} / \mathrm{L}$ & $\mathrm{X}$ & $\mathrm{X}$ & & & & \\
\hline $\mathrm{SO}_{4}$ & Sulfate & $\mathrm{mg} / \mathrm{L}$ & & $\mathrm{X}$ & & & $\mathrm{X}$ & \\
\hline Sp Cond & Specific Conductance & $\mu \mathrm{S} / \mathrm{cm}$ & & $\mathrm{X}$ & & & $\mathrm{X}$ & \\
\hline $\mathrm{Sr}$ & Strontium & $\mu \mathrm{g} / \mathrm{L}$ & $\mathrm{X}$ & $\mathrm{X}$ & & & & \\
\hline $\mathrm{Tl}$ & Thallium & $\mu \mathrm{g} / \mathrm{L}$ & $X$ & & & & & \\
\hline$\underline{\mathrm{U}}$ & Uranium & $\mu \mathrm{g} / \mathrm{L}$ & $\mathrm{X}$ & & & & & \\
\hline V & Vanadium & $\mu \mathrm{g} / \mathrm{L}$ & $\mathrm{X}$ & $\mathrm{X}$ & & & & \\
\hline $\mathrm{Zn}$ & Zinc & $\mu \mathrm{g} / \mathrm{L}$ & $X$ & & & & & \\
\hline
\end{tabular}


Laboratories were requested to identify the method used for each constituent according to analytical method codes in the list of definitions, abbreviations, and symbols (page iv).

Participating laboratories were also asked to identify the method used, such as those references listed next, to further define the methods.

1. American Public Health Association, American Water Works Association, and Water Environment Federation, 1995, Standard methods for the examination of water and wastewater (19th ed.): Washington, D.C., American Public Health Association, variable pagination.

2. American Society for Testing and Materials, 1995, Annual book of ASTM standards: Philadelphia, v. 11.0, and v. 11.02.3.

3. Kopp, J.F., and McKee, G.F., 1979, Methods for chemical analysis of water and wastes: Cincinnati, U.S. Environmental Protection Agency, EPA 600/4-79-020, rev. 1983,460 p.

4. Fishman, M.J., and Friedman, L.C., eds., 1989. Methods for determination of inorganic substances in water and fluvial sediments (3rd ed.): U.S. Geological Survey Techniques of Water-Resources Investigations, Book 5, Chapter A1, 545 p.

5. Miscellaneous manufacturer's instrument manuals or references. 


\section{STATISTICAL PRESENTATION OF DATA}

Data in this report are evaluated using nonparametric statistics as described by Hoaglin and others (1983). This statistical approach is a resistant statistic because outliers have less influence on the median, than does the mean in traditional parametric statistics. Analytical data for each analyte are presented in tabular and graphical forms in tables 11 - 16. Tabulated data for each analyte include the laboratory identification number; reported values; analytical method; most probable value (MPV); number of reported analyses, excluding less than values, $(n)$; data range; the F-pseudosigma; and the $\mathrm{Z}$-value. The $\mathrm{Z}$-value is equivalent to the $\mathrm{Z}$-score of traditional statistics. The F-pseudosigma approximates the standard deviation $(\sigma)$ of traditional statistics when the data has a Gaussian distribution. If an analyte has at least five analyses by a given method, the median and Fpseudosigma are reported in the block of data listed for each method.

The median value calculated from the reported results is the MPV. The F-pseudosigma is calculated by dividing the fourth-spread (analogous to interquartile range) by 1.349 ; therefore the smaller the Fpseudosigma the more precise the determinations. The 1.349 value is derived from the Empirical Rule that defines 1 standard deviation as $67.45 \%$ or 0.6745 of the data; 2 standard deviations contains $95 \%$ or 1.349. Based on an assessment of analyte data (Keith Long, Branch of Quality Systems, verbal comm., 1998), when the F-pseudosigma is less than 5 percent of the MPV, the rating criterion is set to 5 percent of the MPV; as shown in table 11, T-169 Barium.

The graphical plot of the reported data is shown in figure 1. The upper and lower boundaries of the graphical plots are +3 and -3 F-pseudosigma deviations from the median. Reported values are grouped by analytical method in ascending order of value.

The term "inadequate data" is used when the number of analyses is less than seven or the calculated F-pseudosigma is greater than the MPV.

\section{LABORATORY PERFORMANCE RATINGS}

To facilitate laboratory intercomparison, laboratory performance ratings are included in tables 4 - 16 in this report. For each SRS, averages of all the analyte ratings and the number of rated analyses are given for each participating laboratory. The actual reported values by all the laboratories were used to calculate the statistical results and performance ratings presented in this report. Laboratory determination of each analyte is rated on a scale 4 to 0 , based on the absolute Z-value. The listing of ratings and $\mathrm{Z}$-values are presented in the list of analytical methods, abbreviations, and symbols given on page iv and in tables $5-10$.

Laboratories reporting "less than" values are not performance rated unless their less than value is less than the MPV and has a Z-value greater than 2 . In this case, the laboratory would receive a rating of 0 for that analyte.

Ratings are based on the relative performance of laboratories on specific samples and should be reviewed and evaluated on a case-by-case basis for each laboratory considering such factors as methods used and data needs of specific USGS projects using the laboratory data. 


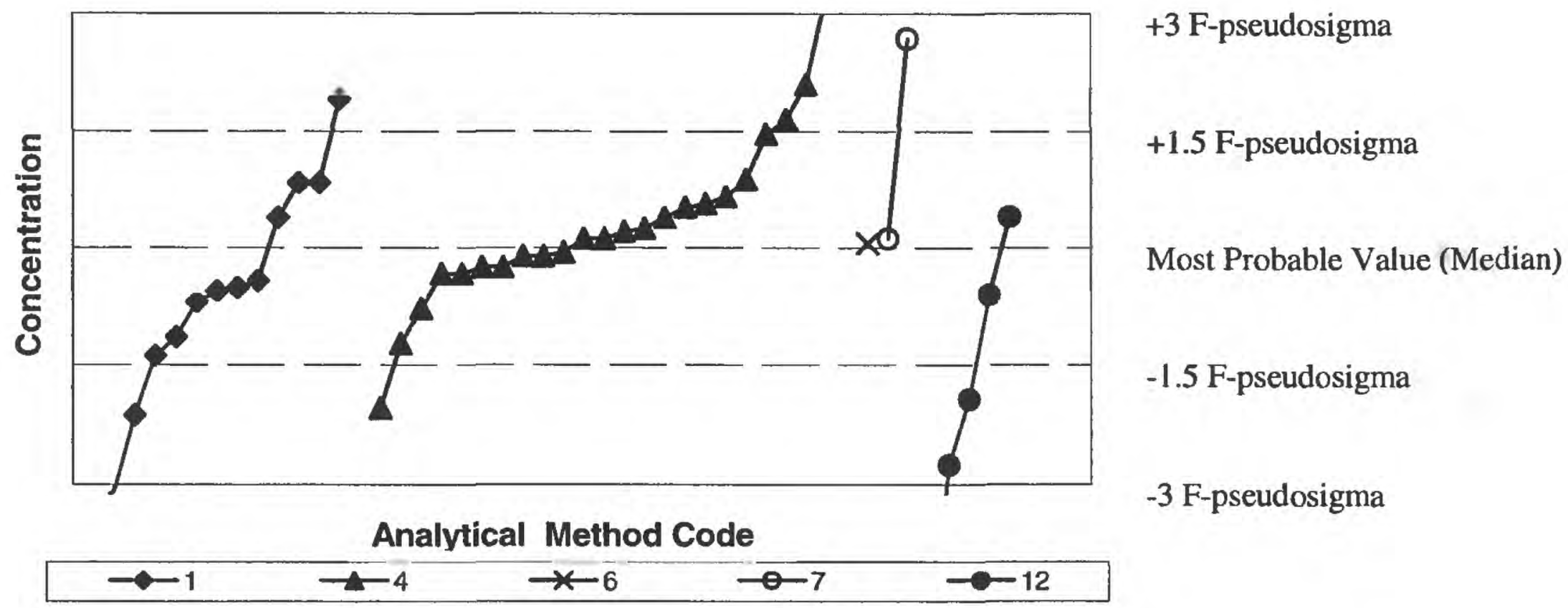

NOTE: vertical scale is the concentration value of the individual analyte in appropriate units (see table 3 ). Horizontal scale is the laboratory reported values separated by method (different symbols) and plotted by increasing values. Numbers next to each symbol at the bottom of the figure are analytical method codes as described on page iv. Laboratory-reported results outside +/- 3 F-pseudosigma from the median are not shown on the graphs.

Figure 1. Statistical parameters shown on data graphs in tables 11-16

\section{REFERENCE}

Hoaglin, D.C., Mosteller, F., and Tukey, J.W., Eds. 1983, Understanding robust and exploratory data analysis: New York, NY, John Wiley, Inc., p. 38-41. 


\section{Table 4. Overall laboratory performance ratings for standard reference samples distributed March 2002}

[SRS, standard reference sample; Lab, laboratory identification number; OWR, overall weighted rating for all sample types; OLR, overall laboratory rating for sample type; V/66, number of rated analyses out of 66 from all sample types; V/28, V/16, V/5, V/5, V/11, and V/1 are number of rated analyses for each sampe type (T-169, M-162, N-73, N-74, P-38, HG-34) respectively; NR, not rated; --, not reported.]

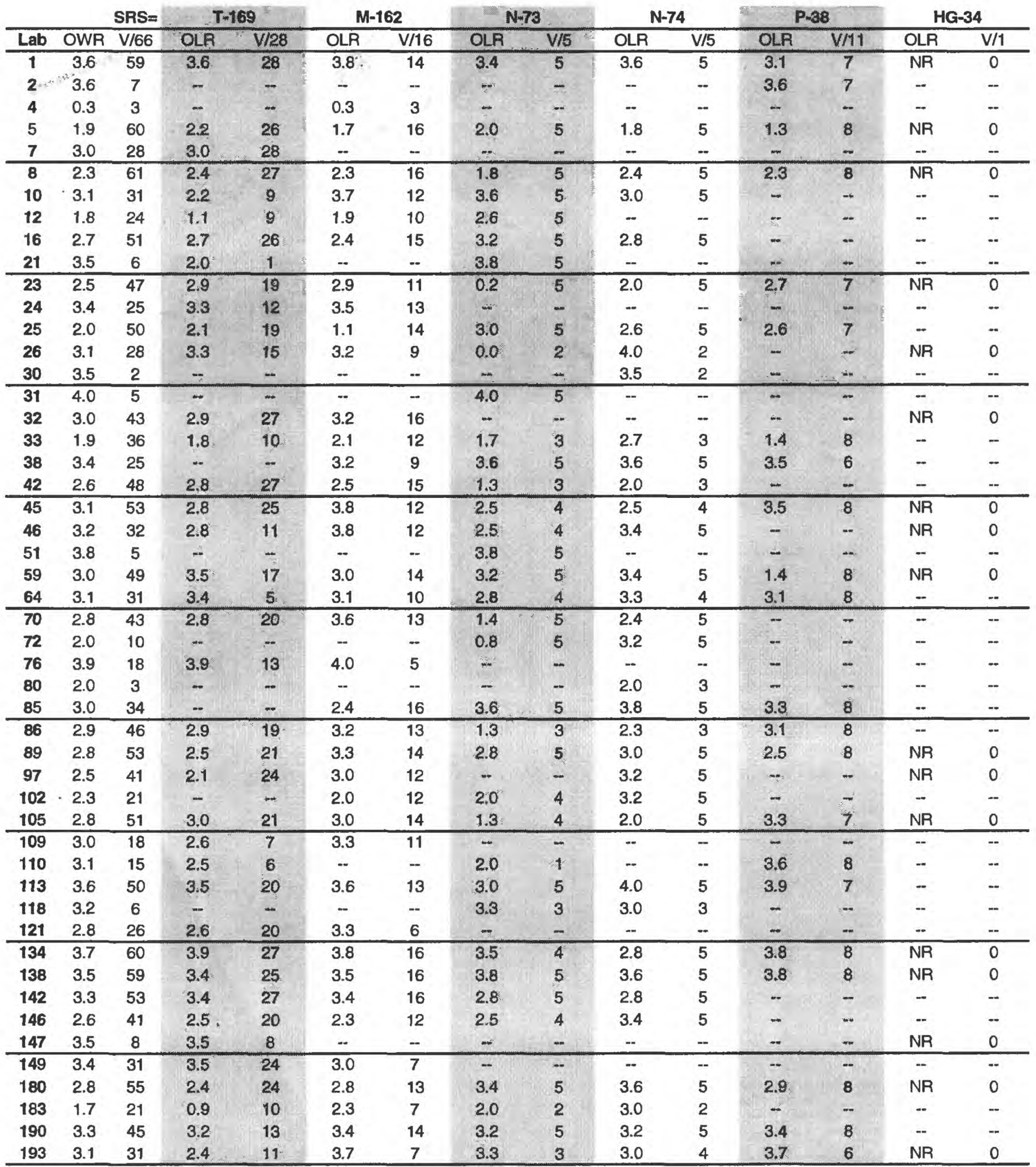


Table 4. Overall laboratory performance ratings for standard reference samples dlstributed March 2002 -continued

[SRS, standard reference sample; Lab, laboratory identification number; OWR, overall weighted rating for all sample types; OLR, overall laboratory rating for sample type; V/66, number of rated analyses out of 66 from all sample types; V/28, V/16, V/5, V/5, V/11, and V/1 are number of rated analyses for each sampe type (T-169, M-162, N-73, N-74, P-38, HG-34) respectively; NR, not rated; --, not reported.]

\begin{tabular}{|c|c|c|c|c|c|c|c|c|c|c|c|c|c|c|}
\hline \multirow[b]{2}{*}{ Lab } & \multirow{2}{*}{ OWR } & \multirow{2}{*}{$\frac{\text { SRS }=}{\text { V/66 }}$} & \multicolumn{2}{|c|}{$T-169$} & \multicolumn{2}{|c|}{$M-162$} & \multicolumn{2}{|c|}{$N * 73$} & \multicolumn{2}{|c|}{$N-74$} & \multicolumn{2}{|c|}{ P.38 } & \multicolumn{2}{|c|}{ HG-34 } \\
\hline & & & OLR & V/28 & OLR & $V / 16$ & OLR & $V / 5$ & OLR & $\mathrm{V} / 5$ & OLR & V/11 & OLR & $V / 1$ \\
\hline 198 & 2.8 & 31 & 2.9 & 23 & -- &.- & 3.0 & 4 & 2.0 & 4 & - & - & -- & -- \\
\hline 205 & 4.0 & 2 & - & - & -- & -- & - & - & 4.0 & 2 & - & - & -. & -- \\
\hline 208 & 3.0 & 5 & - & - & 3.5 & 2 & -- & - & 2.0 & 2 & 4.0 & 1 & - & -- \\
\hline 212 & 2.2 & 40 & $2: 1$ & 22 & 2.4 & 14 & - & -- & 1.8 & 4 & - & - & -- & -- \\
\hline 220 & 3.1 & 17 & 3.0 & 9 & 3.1 & 8 & - & - & -. & - & - & - & -- & -. \\
\hline 224 & 2.8 & 18 & 2 & $=$ & 2.9 & 13 & $\ldots$ & $\overline{-}$ & 2.4 & 5 & - & $=$ & - & -. \\
\hline 227 & 3.1 & 17 & 3.7 & 6 & 3.3 & 6 & 2.2 & 5 & -- & - & - & - & -. & -- \\
\hline 234 & 3.0 & 51 & 3.2 & 27 & 2.8 & 16 & 2.5 & 4 & 2.3 & 4 & - & -. & .. & -- \\
\hline 245 & 3.3 & 31 & 3.1 & 23 & 3.6 & 5 & - & .. & - & -- & 3.7 & 3 & NR & 0 \\
\hline 247 & 2.6 & 57 & 2.6 & 24 & 2.3 & 15 & 3.0 & 5 & 3.6 & 5 & 2.1 & 8 & NR & 0 \\
\hline 255 & 3.3 & 16 & 3.3 & 12 & 3.5 & 4 & - & - & - & - & -. & - & - & - \\
\hline 256 & 2.2 & 35 & 2.4 & 21 & 1.9 & 14 & $\cdots$ & - & - & -- & - & - & -- & -- \\
\hline 259 & 3.4 & 36 & 3.3 & 22 & 3.4 & 14 & -. & - & -- & - & $\ldots$ & - & -. & -- \\
\hline 263 & 3.8 & 10 & $\ldots$ & - & 3.8 & 10 & - & -- & -- & - & $\ldots$ & - & -- & -- \\
\hline 265 & 3.6 & 44 & 3.6 & 28 & 3.4 & 10 & - & $\ldots$ & - & -- & 3.5 & 6 & -- & $\ldots$ \\
\hline 266 & 2.8 & 11 & $\cdots$ & 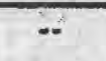 & 2.8 & 11 & - & $\therefore$ & -- & -- & - & 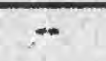 & -. & -- \\
\hline 269 & 2.3 & 7 & - & $\therefore$ & 2.3 & 7 & - & -. & -- & -- & - & .. & -. & - \\
\hline 277 & 3.8 & 4 & - & - & 3.8 & 4 & - & - & -. & - & - & - & -- & -. \\
\hline 279 & 1.9 & 12 & 2.5 & 4 & 1.5 & 4 & - & - & -- & -- & 1.8 & 4 & -- & -- \\
\hline 304 & 3.9 & 14 & $3: 9$ & 14 & -- & - & - & - & -- & $\ldots$ & - & - & NR & 0 \\
\hline 305 & 2.6 & 34 & 2.5 & 22 & 2.2 & 9 & $-x$ & $=$ & 3.7 & 3 & $\ldots$ & - & -- & -. \\
\hline 306 & 1.0 & 8 & - & - & - & - & 0.5 & 4 & 1.5 & 4 & -. & - & -- & -- \\
\hline 313 & 3.8 & 10 & - & - & - & -- & 3.6 & 5 & 4.0 & 5 & $\ldots$ & - & -- & -- \\
\hline 315 & 3.1 & 18 & 2.7 & 6 & 3.5 & 6 & .. & 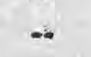 & -- & - & 3.2 & 6 & -- & -. \\
\hline 316 & 1.6 & 5 & $\ldots$ & + & -. & - & 1.6 & 5 & - & -. & $\ldots$ & - & - & $\ldots$ \\
\hline 318 & 3.8 & 5 & $\overline{-}$ & - & $=$ & -- & 3.8 & 5 & -- & - & - & - & - & $\overline{-}$ \\
\hline 319 & 3.0 & 2 & - & - & 3.0 & 2 & - & - & - & - & $=$ & - & -- & -- \\
\hline 320 & 3.2 & 10 & - & - & -. & -- & 3.0 & 5 & 3.4 & 5 & - & - & -- & -- \\
\hline 321 & 1.5 & 23 & - & - & 0.9 & 11 & 2.5 & 4 & -- & -- & 1.9 & 8 & -- & -- \\
\hline 328 & 2.0 & 62 & 2.1 & 28 & 2.1 & 16 & 1.6 & 5 & 1.2 & 5 & 2.4 & 8 & $\cdots$ & -- \\
\hline 330 & 2.5 & 34 & 2.7 & 24 & 2.1 & 10 & - & $\overline{-}$ & - & -- & $\ldots$ & $\omega$ & $=$ & - \\
\hline 333 & 2.9 & 17 & - & - & 2.5 & 8 & 2.0 & 2 & -- & -- & 3.6 & 7 & -- & -. \\
\hline 336 & 0.2 & 16 & - & - & 0.0 & 8 & $\ldots$ & - & -- & -- & 0.4 & 8 & -- & -. \\
\hline 341 & 3.0 & 24 & - & - & 2.9 & 14 & 3.4 & 5 & 3.0 & 5 & - & .. & - & -- \\
\hline 356 & 2.9 & 35 & 2.7 & 24 & 3.8 & 6 & - & - & 3.0 & 5 & - & $=$ & NR & 0 \\
\hline 366 & 3.0 & 21 & - & - & 3.3 & 11 & 3.0 & 5 & 2.6 & 5 & - & $\overline{--}$ & $\overline{--}$ & - \\
\hline 370 & 1.8 & 48 & 1.4 & 18 & 2.2 & 13 & 0.8 & 4 & 2.4 & 5 & 1.9 & 8 & NR & 0 \\
\hline 372 & 1.9 & 58 & 1.9 & 25 & 2.1 & 15 & 1.4 & 5 & 2.4 & 5 & 1.6 & 8 & NR & 0 \\
\hline 373 & 3.4 & 10 & - & - & -. & - & 3.6 & 5 & 3.2 & 5 & - & + & -- & -- \\
\hline 374 & 0.0 & 1 & - & - & 0.0 & 1 & - & - & - & - & 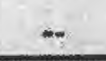 & - & -- & -- \\
\hline 375 & 4.0 & 4 & $\bar{H}$ & 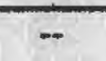 & 4.0 & 4 & $\ldots$ & - & -- & - & .. & 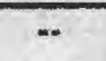 & - & - \\
\hline 376 & 3.3 & 10 & - & - & -. & -- & 3.0 & 5 & 3.6 & 5 & - & -- & -- & -- \\
\hline 377 & 3.4 & 5 & - & $\ldots$ & -- & -. & 3.4 & 5 & -- & -. & - & - & -- & - \\
\hline
\end{tabular}


Table 5. Laboratory performance ratings for standard reference sample T-169 (trace constituents)

IMPV, most probable value; Lab, laboratory Identification number; OLR, overall laboratory rating for all rated analyses; $\mu \mathrm{g} / \mathrm{L}$, micrograms per liter; $\mathrm{mg} / \mathrm{L}$, milligrams per liter; V/28, number of rated analyses out of 28 possible; RV, reported value; <, less than; NR, not rated; --, not reported.]

\begin{tabular}{|lcll|}
\hline Rating & Absolute Z-value & Rating & Absolute Z-value \\
4 (Excellent) & $0.00-0.50$ & 1 (Marginal) & $1.51-2.00$ \\
3 (Good) & $0.51-1.00$ & 0 (Unsatisfactory) & greater than 2.00 \\
2 (Satisfactory) & $1.01-1.50$ & NR (Not Rated) & \\
\hline
\end{tabular}

\begin{tabular}{|c|c|c|c|c|c|c|c|c|c|c|c|c|}
\hline \multirow[b]{2}{*}{ Lab } & \multicolumn{2}{|c|}{$\begin{array}{r}\text { Analyte }= \\
\mathrm{MPV}= \\
\text { F-pseudosigma }=\end{array}$} & \multicolumn{2}{|c|}{$\begin{array}{l}\quad \text { Silver } \\
3.90 \mu \mathrm{g} / \mathrm{L} \\
0.24\end{array}$} & \multicolumn{2}{|c|}{$\begin{array}{l}\text { AlumInum } \\
33.6 \mu \mathrm{g} / \mathrm{L} \\
4.5\end{array}$} & \multicolumn{2}{|c|}{$\begin{array}{l}\text { Arsenic } \\
8.63 \mu \mathrm{g} / \mathrm{L} \\
0.67\end{array}$} & \multicolumn{2}{|c|}{$\begin{array}{c}\text { Boron } \\
24.5 \mu \mathrm{g} / \mathrm{L} \\
1.8\end{array}$} & \multicolumn{2}{|c|}{$\begin{array}{c}\text { Barlum } \\
43.1 \mu \mathrm{g} / \mathrm{L} \\
1.5\end{array}$} \\
\hline & OLR & V/28 & RV & Rating & RV & Rating & RV & Rating & RV & Rating & RV & Rating \\
\hline 1 & 3.6 & 28 & 3.97 & 4 & 33.31 & 4 & 9 & 3 & 25.41 & 3 & 42.94 & 4 \\
\hline 5 & 2.2 & 26 & 6.35 & 0 & 49.9 & 0 & 9.38 & 2 & 24 & 4 & 44.1 & 4 \\
\hline 7 & 3.0 & 28 & $<2$ & 0 & 33.5 & 4 & 8.6 & 4 & 22.9 & 3 & 42.2 & 4 \\
\hline 8 & 2.4 & 27 & 3.65 & 2 & 26.7 & 1 & 6.9 & 0 & 21.7 & 1 & 44.3 & 3 \\
\hline 10 & 2.2 & 9 & $=$ & - & - & $\cdots$ & 9.3 & 3 & -- & -- & - & - \\
\hline 12 & 1.1 & 9 & 3.7 & 3 & - & - & 8 & 3 & - & - & - & - \\
\hline 16 & 2.7 & 26 & 4 & 4 & 29 & 2 & 10 & 0 & 13 & 0 & 41 & 3 \\
\hline 21 & 2.0 & 1 & - & $\rightarrow$ & -. & -. & - & - & -- & -. & - & - \\
\hline 23 & 2.9 & 19 & 3.52 & 1 & -- & -- & 10.31 & 0 & -- & -- & 43.07 & 4 \\
\hline 24 & 3.3 & 12 & - & 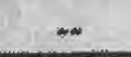 & - & -- & - & - & 23.8 & 4 & 45.4 & 2 \\
\hline 25 & 2.1 & 19 & रा7 & NR & 290 & 0 & 8.1 & 3 & 26 & 3 & 35 & 0 \\
\hline 26 & 3.3 & 15 & - & - & 35.2 & 4 & 8.3 & 4 & - & -. & - & - \\
\hline 32 & 2.9 & 27 & 3.9 & 4 & 31.1 & 3 & 8.6 & 4 & 23.6 & 4 & 42.8 & 4 \\
\hline 33 & 1.8 & 10 & $\ldots$ & - & 53 & 0 & -. & - & -- & -- & 45.1 & 3 \\
\hline 42 & 2.8 & 27 & 3.6 & 2 & 29.7 & 3 & 8.46 & 4 & 23.9 & 4 & 43.2 & 4 \\
\hline 45 & 2.8 & 25 & 4.8 & 0 & 33.6 & 4 & 8.93 & 4 & 27.5 & 1 & 41.9 & 3 \\
\hline 46 & 2.8 & 11 & - & - & -- & - & 6.57 & 0 & -- & - & 43.1 & 4 \\
\hline 59 & 3.5 & 17 & $<5$ & NR & $<50$ & NR & 8.76 & 4 & 21.3 & 1 & 43.1 & 4 \\
\hline 64 & 3.4 & 5 & - & - & -- & - & - & - & -- & -. & - & - \\
\hline 70 & 2.8 & 20 & - & - & 28.7 & 2 & 10.4 & 0 & $<100$ & $N R$ & 43.1 & 4 \\
\hline 76 & 3.9 & 13 & $\overline{-}$ & $\ldots$ & 33.08 & 4 & 8.627 & 4 & $\ldots$ & -. & - & $\ldots$ \\
\hline 86 & 2.9 & 19 & 2.45 & 0 & -. & - & - & - & 26.2 & 3 & 41.7 & 3 \\
\hline 89 & 2.5 & 21 & 3.8 & 4 & 38 & 3 & 8.7 & 4 & -- & -- & 58.9 & 0 \\
\hline 97 & 2.1 & 24 & 4.01 & 4 & 41 & 1 & 10.6 & 0 & -- & - & 42.3 & 4 \\
\hline 105 & 3.0 & 21 & 3.58 & 2 & 33.69 & 4 & 9.54 & 2 & $<200$ & NR & 43 & 4 \\
\hline 109 & 2.6 & 7 & - & - & - & - & 9.19 & 3 & - & - & - & 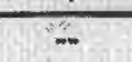 \\
\hline 110 & 2.5 & 6 & - & - & 34.098 & 4 & - & - & -- & -. & - & - \\
\hline 113 & 3.5 & 20 & 4.03 & 3 & 31.6 & 4 & 8.67 & 4 & -- & -- & 42.9 & 4 \\
\hline 121 & 2.6 & 20 & 3.4 & 0 & -- & -- & 7 & 0 & .. & -- & 47 & 1 \\
\hline 134 & 3.9 & 27 & 3.9 & 4 & 33.25 & 4 & 8.84 & 4 & 25.29 & 4 & 43.84 & 4 \\
\hline 138 & 3.4 & 25 & 3.82 & 4 & 36.4 & 3 & 8.98 & 3 & 22.3 & 2 & 43.5 & 4 \\
\hline 142 & 3.4 & 27 & 3.72 & 3 & 37.6 & 3 & 9.05 & 3 & 26.9 & 2 & 44.9 & 3 \\
\hline 146 & 2.5 & 20 & 3.59 & 2 & 63.2 & 0 & 9.82 & 1 & -- & -- & 44 & 4 \\
\hline 147 & 3.5 & 8 & - & - & -- & -. & 8.49 & 4 & -- & -- & .. & - \\
\hline 149 & 3.5 & 24 & 4.1 & 3 & 33.6 & 4 & 8.9 & 4 & -- & -- & 44,1 & 4 \\
\hline 180 & 2.4 & 24 & 3.37 & 0 & 28.8 & 2 & 8.26 & 3 & $<3.25$ & 0 & 41.5 & 3 \\
\hline 183 & 0.9 & 10 & - & - & -- & -- & 6.09 & 0 & -- & - & 43.1 & 4 \\
\hline 190 & 3.2 & 13 & 3.93 & 4 & 32 & 4 & 7.5 & 1 & -- & -. & .. & - \\
\hline 193 & 2.4 & 11 & 4.24 & 2 & -- & -- & 8.07 & 3 & -- & - & - & - \\
\hline 198 & 2.9 & 23 & 3.73 & 3 & 28.7 & 2 & 8.59 & 4 & .. & -- & 41,7 & 3 \\
\hline 212 & 2.1 & 22 & 4.1 & $\overline{3}$ & 20.3 & 0 & 8.8 & 4 & - & -- & 46.2 & 2 \\
\hline 220 & 3.0 & 9 & - & . & -- & -- & 8.2 & 3 & -- & - & 47.06 & 1 \\
\hline 227 & 3.7 & 6 & .. & - & -- & -- & - & - & -- & -- & -. & - \\
\hline 234 & 3.2 & 27 & 3.77 & 3 & 40 & 2 & 8.74 & 4 & 27.9 & 1 & 43.9 & 4 \\
\hline 245 & 3.1 & 23 & 3.568 & 2 & 33.8 & 4 & 8.64 & 4 & -- & -- & 41.29 & 3 \\
\hline
\end{tabular}


Table 5. Laboratory performance ratings for standard reference sample T-169 (trace constituents) -- continued [MPV, most probable value; Lab, laboratory identification number; OLR, overall laboratory rating for all rated analyses; $\mu \mathrm{g} / \mathrm{L}$, micrograms per liter; $\mathbf{m g} / \mathrm{L}$, milligrams per liter; V/28, number of rated analyses out of 28 possible; RV, reported value; <, less than; NR, not rated; --, not reported.]

\begin{tabular}{|lcll|}
\hline Rating & Absolute Z-value & \multicolumn{1}{c|}{ Rating } & Absolute Z-value \\
4 (Excellent) & $0.00-0.50$ & 1 (Marginal) & $1.51-2.00$ \\
3 (Good) & $0.51-1.00$ & ( Unsatisfactory) & greater than 2.00 \\
2 (Satisfactory) & $1.01-1.50$ & NR (Not Rated) & \\
\hline
\end{tabular}

\begin{tabular}{|c|c|c|c|c|c|c|c|c|c|c|c|c|}
\hline \multirow[b]{2}{*}{ Lab } & \multicolumn{2}{|c|}{$\begin{array}{r}\text { Analyte }= \\
\mathrm{MPV}= \\
\text { F-pseudosigma }=\end{array}$} & \multicolumn{2}{|c|}{$\begin{array}{l}\text { Silver } \\
3.90 \mu g / L \\
0.24 \\
\end{array}$} & \multicolumn{2}{|c|}{$\begin{array}{l}\text { Aluminum } \\
33.6 \mu \mathrm{g} / \mathrm{L} \\
4.5\end{array}$} & \multicolumn{2}{|c|}{$\begin{array}{l}\text { Arsenic } \\
8.63 \mu \mathrm{g} / \mathrm{L} \\
0.67\end{array}$} & \multicolumn{2}{|c|}{$\begin{array}{c}\text { Boron } \\
24.5 \mu \mathrm{g} / \mathrm{L} \\
1.8 \\
\end{array}$} & \multicolumn{2}{|c|}{$\begin{array}{l}\text { Barlum } \\
43.1 \mu \mathrm{g} / \mathrm{L} \\
1.5\end{array}$} \\
\hline & OLR & V/28 & AV & Rating & RV & Rating & RV & Rating & RV & Rating & RV & Rating \\
\hline 247 & 2.6 & 24 & 3.78 & 4 & 32.6 & 4 & 7.36 & 1 & 80 & 0 & 41.9 & 3 \\
\hline 255 & 3.3 & 12 & 4 & 4 & - & -. & 8.4 & 4 & 24.7 & 4 & - & - \\
\hline 256 & 2.4 & 21 & 3.96 & 4 & 35.75 & 4 & 2.8 & 0 & - & -- & 44.4 & 3 \\
\hline 259 & 3.3 & 22 & 3.9 & 4 & 37 & 3 & 7.6 & 1 & 25.3 & 4 & 43.7 & 4 \\
\hline 265 & 3.6 & 28 & 3.8 & 4 & 35 & 4 & 9.1 & 3 & 24.5 & 4 & 43 & 4 \\
\hline 279 & 2.5 & 4 & - & " & - & - & - & - & - & - & - & - \\
\hline 304 & 3.9 & 14 & 3,97 & 4 & 31.9 & 4 & - & -. & - & -. & 43.4 & 4 \\
\hline 305 & 2.5 & 22 & 4.1 & 3 & 52 & 0 & 9 & 3 & -- & -- & 42 & 3 \\
\hline 315 & 2.7 & 6 & . & - & - & - & -. & -. & $\cdot$ & - & -. & - \\
\hline 328 & 2.1 & 28 & 6.6 & 0 & 26 & 1 & 8.1 & 3 & 24 & 4 & 43 & 4 \\
\hline 330 & 2.7 & 24 & 4 & 4 & 33.6 & 4 & 9.3 & 3 & - & - & 42.2 & 4 \\
\hline 356 & 2.7 & 24 & 3.74 & 3 & 43 & 0 & 8.55 & 4 & -- & - & 41.6 & 3 \\
\hline 370 & 1.4 & 18 & 6.47 & 0 & $<100$ & NR & 6.14 & 0. & -- & - & 52.7 & 0 \\
\hline 372 & 1.9 & 25 & 3 & 0 & 39 & 2 & B & 3 & -- & -- & 42 & 3 \\
\hline
\end{tabular}


Table 5. Laboratory performance ratings for standard reference sample T-169 (trace constituents) -- continued [MPV, most probable value; Lab, laboratory identification number; OLR, overall laboratory rating for all rated analyses; $\mu \mathrm{g} / \mathrm{L}$, micrograms per liter, $\mathrm{mg} / \mathrm{L}$, milligrams per liter; V/28, number of rated analyses out of 28 possible; RV, reported value; <, less than; NR, not rated; --, not reported.]

\begin{tabular}{|lcll|}
\hline Rating & Absolute Z-value & Rating & Absolute Z-value \\
4 (Excellent) & $0.00-0.50$ & 1 (Marginal) & $1.51-2.00$ \\
3 (Good) & $0.51-1.00$ & 0 (Unsatisfactory) & greater than 2.00 \\
2 (Satisfactory) & $1.01-1.50$ & NR (Not Rated) & \\
\hline
\end{tabular}

\begin{tabular}{|c|c|c|c|c|c|c|c|c|c|c|c|}
\hline \multirow[b]{2}{*}{ Lab } & $\begin{array}{r}\text { Analyte }= \\
\mathrm{MPV}= \\
\text { F-pseudosigma }=\end{array}$ & \multicolumn{2}{|c|}{$\begin{array}{l}\text { Beryillum } \\
9.61 \mu \mathrm{g} / \mathrm{L} \\
0.56\end{array}$} & \multicolumn{2}{|c|}{$\begin{array}{l}\text { Calclum } \\
37.6 \mathrm{mg} / \mathrm{L} \\
0.9\end{array}$} & \multicolumn{2}{|c|}{$\begin{array}{l}\text { Cadmilum } \\
3.40 \mu \mathrm{g} / \mathrm{L} \\
0.21\end{array}$} & \multicolumn{2}{|c|}{$\begin{array}{l}\text { Cobalt } \\
1.91 \mu \mathrm{g} / \mathrm{L} \\
0.17\end{array}$} & \multicolumn{2}{|c|}{$\begin{array}{l}\text { Chromium } \\
8.17 \mu g / \\
0.39\end{array}$} \\
\hline & & RV & Rating & RV & Rating & RV & Rating & RV & Rating & RV & Rating \\
\hline 1 & & 9.53 & 4 & 38.02 & 4 & 3.61 & 3 & 2.03 & 3 & 8.75 & 2 \\
\hline 5 & & 9.38 & 4 & 37.7 & 4 & 3.22 & 3 & $<3.00$ & NR & 8.94 & 1 \\
\hline 7 & & 8.8 & 2 & 36.6 & 3 & 3.2 & 3 & 10.2 & 0 & 8.4 & 3 \\
\hline 8 & & $8: 9$ & 2 & 40.6 & 1 & 3.7 & 2 & 2 & 3 & 8.2 & 4 \\
\hline 10 & & - & - & -- & - & 3.5 & 4 & - & -- & 9.1 & 0 \\
\hline 12 & & - & - & 38 & 4 & 4 & 0 & - & - & - & - \\
\hline 16 & & 10 & 3 & 37 & 4 & 4 & 0 & 2.2 & 1 & 8 & 4 \\
\hline 21 & & - & - & -. & -- & - & - & -- & -. & - & - \\
\hline 23 & & 9.81 & 4 & 36.45 & 3 & 3.38 & 4 & -- & -- & 8 & 4 \\
\hline 24 & & - & - & 36.9 & 4 & - & - & .. & .. & - & - \\
\hline 25 & & 10 & 3 & 34.6 & 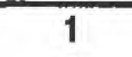 & 3.4 & 4 & $<4$ & $\overline{\mathrm{NR}}$ & $<15$ & NR \\
\hline 26 & & 9.18 & 3 & 37.9 & 4 & 3.45 & 4 & - & - & - & - \\
\hline 32 & & 8.7 & 1 & 36.9 & 4 & 3.6 & 3 & 2 & 3 & 8.5 & 3 \\
\hline 33 & & - & -. & 33.2 & 0 & - & -. & -- & -- & - & - \\
\hline 42 & & 9.73 & 4 & 35.6 & 2 & 3.18 & 2 & $<2$ & NR & 7.95 & 3 \\
\hline 45 & & 9.28 & 3 & 37.3 & 4 & 3.15 & 2 & 1.91 & 4 & 7.83 & $\overline{3}$ \\
\hline 46 & & 9.68 & 4 & 38.1 & 4 & 3.28 & 3 & - & -- & 7.36 & 1 \\
\hline 59 & & 9.74 & 4 & 38 & 4 & $<5$ & NR & $<5$ & NR & $<10$ & NR \\
\hline 64 & & - & - & 38.6 & 3 & - & - & -- & -- & - & $\pi$ \\
\hline 70 & & 10.3 & 2 & 37.7 & 4 & 3.51 & 3 & $<10$ & NR & 8.27 & 4 \\
\hline 76 & & - & 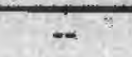 & 37.89 & 4 & 3.404 & 4 & - & -- & - & $\overline{-}$ \\
\hline 86 & & 9.08 & 3 & 37.6 & 4 & 3.08 & 2 & -- & -- & 6.7 & 0 \\
\hline 89 & & 8.3 & 0 & 39.6 & 2 & 4 & 0 & 1.9 & 4 & 8.3 & 4 \\
\hline 97 & & 9.88 & 4 & 37.9 & 4 & 3.51 & 3 & 2.65 & 0 & 8.96 & 1 \\
\hline 105 & & 9 & 2 & 35.2 & 2 & 3.32 & 4 & $<50$ & NR & 8.13 & 4 \\
\hline 109 & & $\ldots$ & 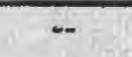 & 36.42 & 3 & - & - & - & - & - & 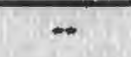 \\
\hline 110 & & - & -. & 36.553 & 3 & - & - & -- & -- & - & - \\
\hline 113 & & 10.1. & 3 & 37.3 & 4 & 3.24 & 3 & -. & - & 8.17 & 4 \\
\hline 121 & & 9.4 & 4 & 38.2 & 4 & 2 & 0 & 0.35 & 0 & 8 & 4 \\
\hline 134 & & 9.23 & 3 & 37.65 & 4 & 3.33 & 4 & 1.64 & 4 & 7.76 & 3 \\
\hline 138 & & 9.58 & 4 & 36.6 & 3 & 3.57 & 3 & 1.95 & 4 & 7.5 & 1 \\
\hline 142 & & 9.82 & 4 & 36.8 & 4 & 3.67 & 2 & 1.88 & 4 & 8.04 & 4 \\
\hline 146 & & 9.11 & 3 & 36.6 & 3 & 3.22 & 3 & 2.38 & 0 & 8.1 & 4 \\
\hline 147 & & -. & - & -- & -- & 3.19 & 3 & 1.71 & 2 & - & - \\
\hline 149 & & 9.7 & 4 & 37.4 & 4 & 3.4 & 4 & 1.9 & 4 & 8 & 4 \\
\hline 180 & & 9.07 & 3 & 36.9 & 3 & 3.29 & 3 & 1.73 & 2 & 7.52 & T \\
\hline 183 & & 9.14 & 3 & -- & -- & 3.83 & 0 & -- & -- & 8.79 & 1 \\
\hline 190 & & - & - & -- & -- & 3.28 & 3 & - & .. & 8.5 & 3 \\
\hline 193 & & 9.5 & 4 & 41.1 & 1 & 3.39 & 4 & -- & -- & 8.64 & 2 \\
\hline 198 & & 9.72 & 4 & 37.7 & 4 & 3.62 . & 2 & 1.8 & 3 & 7.53 & 1. \\
\hline 212 & & 9.5 & 4 & 36.8 & 4 & 3.1 & 2 & 1.1 & 0 & 8.7 & 2 \\
\hline 220 & & 9.63 & 4 & 38.51 & 4 & . & $\ldots$ & -- & .. & - & - \\
\hline 227 & & - & -. & 38.4 & 4 & 3.39 & 4 & -. & .- & .. & - \\
\hline 234 & & 9.93 & 3 & 36 & 4 & 3.3 & 4 & 1.95 & 4 & 8.49 & 3 \\
\hline 245 & & 10.02 & 3 & 37.6 & 4 & 3.654 & 2 & 2.24 & 1 & 8.29 & 4 \\
\hline
\end{tabular}


Table 5. Laboratory performance ratings for standard reference sample T-169 (trace constituents) -- continued [MPV, most probable value; Lab, laboratory identification number; OLR, overall laboratory rating for all rated analyses; $\mu \mathrm{g} / \mathrm{L}$, micrograms per liter; mg/L, milligrams per liter; V/28, number of rated analyses out of 28 possible; RV, reported value; <, less than; NR, not rated; --, not reported.]

\begin{tabular}{|lcll|}
\hline Rating & Absolute Z-value & Rating & Absolute Z-value \\
4 (Excellent) & $0.00-0.50$ & 1 (Marginal) & $1.51-2.00$ \\
3 (Good) & $0.51-1.00$ & 0 (Unsatisfactory) & greater than 2.00 \\
2 (Satisfactory) & $1.01-1.50$ & NR (Not Rated) & \\
\hline
\end{tabular}

\begin{tabular}{|c|c|c|c|c|c|c|c|c|c|c|c|}
\hline \multirow[b]{2}{*}{ Lab } & \multirow{2}{*}{$\begin{array}{r}\text { Analyte }= \\
\text { MPV }= \\
\text { F-pseudosigma }=\end{array}$} & \multicolumn{2}{|c|}{$\begin{array}{l}\text { Beryllium } \\
9.61 \mu \mathrm{g} / \mathrm{L} \\
0.56\end{array}$} & \multicolumn{2}{|c|}{$\begin{array}{l}\text { Calclum } \\
37.6 \mathrm{mg} / \mathrm{L} \\
0.9\end{array}$} & \multicolumn{2}{|c|}{$\begin{array}{l}\text { Cadmium } \\
3.40 \mu \mathrm{g} / \mathrm{L} \\
0.21\end{array}$} & \multicolumn{2}{|c|}{$\begin{array}{l}\text { Cobalt } \\
1.91 \mu \mathrm{g} / \mathrm{L} \\
0.17\end{array}$} & \multicolumn{2}{|c|}{$\begin{array}{l}\text { Chromlum } \\
8.17 \mu \mathrm{g} / \mathrm{L} \\
0.39\end{array}$} \\
\hline & & RV & Rating & $\mathrm{RV}$ & Rating & RV & Rating & $R V$ & Rating & RV & Rating \\
\hline 247 & & 9.71 & 4 & 34.3 & 1 & 3.42 & 4 & 1.77 & 3 & 7.75 & 2 \\
\hline 255 & & - & - & -- & -- & 3.51 & 3 & -- & - & 8.62 & 2 \\
\hline 256 & & 10.15 & 3 & -- & - & 3.48 & 4 & 2 & 3 & 8.09 & 4 \\
\hline 259 & & -- & - & 38 & 4 & 3.4 & 4 & 1.75 & 3 & 8.1 & 4 \\
\hline 265 & & 10.5 & 1 & 38.1 & 4 & 3.6 & 3 & 1.8 & 3 & 8 & 4 \\
\hline 279 & & - & - & 37.34 & 4 & - & - & - & $\cdots$ & - & - \\
\hline 304 & & - & - & - & - & 3.46 & 4 & 1.89 & 4 & 8.4 & 3 \\
\hline 305 & & 10.3 & 2 & 37.5 & 4 & 3.4 & 4 & 2 & 3 & 7.1 & 0 \\
\hline 315 & & -. & - & 38.2 & 4 & - & -- & -. & - & -. & - \\
\hline 328 & & 9.2 & 3 & 37 & 4 & 3.3 & 4 & 1.1 & 0 & 7.8 & 3 \\
\hline 330 & & 11 & 0 & 38.3 & 4 & 3.7 & 2 & 2.1 & 2 & 8.7 & 2 \\
\hline 356 & & 9.38 & 4 & 36.6 & 3 & 3.53 & 3 & 1.9 & 4 & 8.18 & 4 \\
\hline 370 & & 10 & 3 & 40.9 & 1 & 3.48 & 4 & $<500$ & NR & 8.18 & 4 \\
\hline 372 & & 9 & 2 & 37 & 4 & 3 & 1 & 3 & 0 & 8.7 & 2 \\
\hline
\end{tabular}


Table 5. Laboratory performance ratings for standard reference sample T-169 (trace constituents) -- continued [MPV, most probable value; Lab, laboratory identification number; OLR, overall laboratory rating for all rated analyses; $\mu \mathrm{g} / \mathrm{L}$, micrograms per liter; $\mathrm{mg} / \mathrm{L}$, milligrams per liter; V/28, number of rated analyses out of 28 possible; RV, reported value; <, less than; NR, not rated; --, not reported.]

\begin{tabular}{|llll|}
\hline Rating & Absolute Z-value & Rating & Absolute Z-value \\
4 (Excellent) & $0.00-0.50$ & 1 (Marginal) & $1.51-2.00$ \\
3 (Good) & $0.51 \cdot 1.00$ & 0 (Unsatisfactory) & greater than 2.00 \\
2 (Satisfactory) & $1.01-1.50$ & NR (Not Rated) & \\
\hline
\end{tabular}

\begin{tabular}{|c|c|c|c|c|c|c|c|c|c|c|c|}
\hline \multirow{2}{*}{\multicolumn{2}{|c|}{$\begin{array}{r}\text { Analyte }= \\
\text { MPV }= \\
\text { F-pseudosigma }=\end{array}$}} & \multicolumn{2}{|c|}{$\begin{array}{l}\text { Copper } \\
14.3 \mu g / L \\
0.8\end{array}$} & \multicolumn{2}{|c|}{$\begin{array}{l}\text { Iron } \\
11.1 \mu \mathrm{g} / \mathrm{L} \\
3.6\end{array}$} & \multicolumn{2}{|c|}{$\begin{array}{l}\text { Potasslum } \\
2.59 \mathrm{mg} / \mathrm{L} \\
0.11\end{array}$} & \multicolumn{2}{|c|}{$\begin{array}{l}\text { Lithium } \\
9.60 \mu \mathrm{g} / \mathrm{L} \\
0.61\end{array}$} & \multicolumn{2}{|c|}{$\begin{array}{l}\text { Magnesium } \\
4.30 \mathrm{mg} / \mathrm{L} \\
0.13\end{array}$} \\
\hline & & RV & Rating & RV & Rating & RV & Rating & RV & Rating & RV & Rating \\
\hline 1 & & 14.83 & 3 & 11.05 & 4 & 2.6 & 4 & 9.99 & 3 & 4.16 & 3 \\
\hline 5 & & 14.8 & 3 & 13.4 & 3 & 2.81 & 1 & 9.23 & 3 & 4.2 & 4 \\
\hline 7 & & 14.8 & 3 & 13.3 & 3 & 2.62 & 4 & 9.3 & 4 & 4.19 & 3 \\
\hline 8 & & 15.8 & 1 & $<50$ & NR & 2.7 & 3 & 9.3 & 4 & 4.4 & 4 \\
\hline 10 & & 12.9 & 1 & 18 & 1 & -- & - & -- & - & - & - \\
\hline 12 & & 16 & 0 & - & - & - & - & $\overline{-}$ & - & 4,9 & 0 \\
\hline 16 & & 10 & 0 & 10 & 4 & 2.6 & 4 & - & - & 4.4 & 4 \\
\hline 21 & & - & - & 14.9 & 2 & - & - & -- & - & -- & - \\
\hline 23 & & 14.67 & 4 & 11.24 & 4 & 2.59 & 4 & - & - & 4.22 & 4 \\
\hline 24 & & 18.1 & 0 & -- & - & 2.55 & 4 & - & - & 4.16 & 3 \\
\hline 25 & & 14 & 4 & 16 & 2 & 2.47 & 3 & $<4$ & 0 & 4.37 & 4 \\
\hline 26 & & 13.8 & 3 & 13.2 & 3 & 2.48 & 3 & 7.61 & 0 & 4.32 & 4 \\
\hline 32 & & 14.2 & 4 & - & -- & 2.45 & 2 & 8.8 & 2 & 4.3 & 4 \\
\hline 33 & & - & - & 10 & 4 & 2.69 & 3 & -- & - & 4.48 & 3 \\
\hline 42 & & 13.4 & 2 & 16.1 & 2 & 2.32 & 0 & 9.28 & 3 & 4 & 2 \\
\hline 45 & & 14.3 & $\overline{4}$ & 12.8 & 4 & 2.57 & 4 & -- & - & 4.27 & 4 \\
\hline 46 & & 13.1 & 2 & -- & - & -. & - & -- & - & 4.19 & 3 \\
\hline 59 & & 14.2 & 4 & $<100$ & NR & 2.58 & 4 & 9.07 & 3 & 4.26 & 4 \\
\hline 64 & & -4 & - & - & - & 2.58 & 4 & -- & -. & 4.15 & 3 \\
\hline 70 & & 13.7 & 3 & $<20$ & NR & 2.59 & 4 & - & - & 4.25 & 4 \\
\hline 76 & & - & 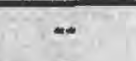 & - & - & 2.596 & 4 & - & $\overline{--}$ & 4.249 & 4 \\
\hline 86 & & 15.5 & 2 & -- & - & 2.65 & 4 & 9.6 & 4 & 4.36 & 4 \\
\hline 89 & & 14.4 & 4 & 9.8 & 4 & 2.61 & 4 & -- & -- & 4,41 & 3 \\
\hline 97 & & 16 & 0 & $<1.8$ & 0 & 2.52 & 3 & -- & -. & 4.33 & 4 \\
\hline 105 & & 15 & 3 & $<20.0$ & NR & 2.65 & 4 & 42 & 0 & 4.3 & 4 \\
\hline 109 & & $\cdots$ & - & $<30$ & NR & 2.3 & 0 & - & - & 4.37 & 4 \\
\hline 110 & & - & $\cdots$ & - & - & 2.786 & 1 & - & - & 4.825 & 0 \\
\hline 113 & & 15.2 & 2 & 11 & 4 & 2.48 & 3 & - & $\sim$ & 4.19 & 3 \\
\hline 121 & & 14 & 4 & 15 & 2 & - & -- & -- & -- & 4.2 & 4 \\
\hline 134 & & 13.91 & 4 & 10.35 & 4 & 2.56 & 4 & 9.6 & 4 & 4.18 & 3 \\
\hline 138 & & 13.6 & 3 & 10.4 & 4 & 2.47 & 3 & -- & -- & 4.32 & 4 \\
\hline 142 & & 13.4 & 2 & -- & -- & 2.44 & 2 & 9.45 & 4 & 4.17 & 3 \\
\hline 146 & & 13.3 & 2 & $<50.0$ & NR & 2.99 & 0 & - & -- & 4.32 & 4 \\
\hline 147 & & 14.2 & 4 & -- & -- & - & - & -- & -- & - & $\ldots$ \\
\hline 149 & & 14.9 & 3 & 111.8 & 0 & 2.6 & 4 & - & -- & 4.4 & 4 \\
\hline 180 & & 13.3 & 2 & 10.5 & 4 & 2.5 & 3 & -- & -- & 4.33 & 4 \\
\hline 183 & & - & - & - & - & - & - & -- & -- & - & - \\
\hline 190 & & 14.2 & 4 & 9.34 & 4 & - & - & - & $\cdots$ & - & - \\
\hline 193 & & 16 & 0 & $<100$ & NR & 2.66 & 3 & - & - & 4.23 & 4 \\
\hline 198 & & 13 & 1. & 17 & 1 & 2.61 & 4 & - & -. & 4.33 & 4 \\
\hline 212 & & 12.4 & 0 & $<100$ & NR & 2.8 & 1 & - & -- & 4.3 & 4 \\
\hline 220 & & - & - & - & -- & - & - & -. & -- & 4.372 & 4 \\
\hline 227 & & 13.2 & 2 & -- & - & - & $=$ & -- & - & 4.25 & 4 \\
\hline 234 & & 14.6 & 4 & 12.6 & 4 & 2.56 & 4 & 10.1 & 3 & 4.4 & 4 \\
\hline 245 & & 15.64 & 1 & - & - & 2.584 & 4 & - & - & 4.409 & 4 \\
\hline
\end{tabular}


Table 5. Laboratory performance ratings for standard reference sample T-169 (trace constituents) -- continued [MPV, most probable value; Lab, laboratory identification number; OLR, overall laboratory rating for all rated analyses; $\mu \mathrm{g} / \mathrm{L}$, micrograms per liter; mg/L, milligrams per liter; V/28, number of rated analyses out of 28 possible; RV, reported value; <, less than; NR, not rated; --. not reported.]

\begin{tabular}{|c|c|c|c|}
\hline Rating & Absolute Z-value & Rating & Absolute Z-value \\
\hline 4 (Excellent) & $0.00=0.50$ & 1 (Marginal) & $1.51-2.00$ \\
\hline 3 (Good) & $0.51-1.00$ & $\mathrm{O}$ (Unsatisfactory) & greater than 2.00 \\
\hline 2 (Satisfactory) & $1.01-1.50$ & NR (Not Rated) & \\
\hline
\end{tabular}

\begin{tabular}{|c|c|c|c|c|c|c|c|c|c|c|c|}
\hline \multirow[b]{2}{*}{ Lab } & \multirow{2}{*}{$\begin{array}{r}\text { Analyta }= \\
\text { MPV }= \\
\text { F-pseudosigma }= \\
\end{array}$} & \multicolumn{2}{|c|}{$\begin{array}{l}\text { Copper } \\
14.3 \mu g / 2 \\
0.8 \\
\end{array}$} & \multicolumn{2}{|c|}{$\begin{array}{l}\text { Iron } \\
11.1 \mu \mathrm{g} / \mathrm{L} \\
3.6 \\
\end{array}$} & \multicolumn{2}{|c|}{$\begin{array}{l}\text { Potasslum } \\
2.59 \mathrm{mg} / \mathrm{L} \\
0.11 \\
\end{array}$} & \multicolumn{2}{|c|}{$\begin{array}{l}\text { Lithlum } \\
9.60 \mu \mathrm{g} / \mathrm{L} \\
0.61 \\
\end{array}$} & \multicolumn{2}{|c|}{$\begin{array}{l}\text { Magnesium } \\
4.30 \mathrm{mg} / \mathrm{L} \\
0.13 \\
\end{array}$} \\
\hline & & RV & Rating & RV & Rating & RV & Rating & RV & Rating & $\mathrm{RV}$ & Rating \\
\hline 247 & & 13.9 & 4 & - & - & 2.37 & 1 & 9.67 & 4 & 3.94 & 1 \\
\hline 255 & & 14.5 & 4 & 14 & 3 & 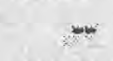 & - & -- & - & - & - \\
\hline 256 & & 18 & 0 & 10.85 & 4 & - & - & 10.5 & 2 & - & $=$ \\
\hline 259 & & 14.4 & 4 & 9.4 & 4 & 2.09 & 0 & 10.7 & 1 & 4.3 & 4 \\
\hline 265 & & 14.4 & 4 & 10 & 4 & 2.6 & 4 & 9.8 & 4 & 4.2 & 4 \\
\hline 279 & & $-m$ & $\ldots$ & - & $\ldots$ & 2.65 & 4 & -- & - & 4.85 & 0 \\
\hline 304 & & 14.3 & 4 & - & - & - & - & - & - & - & - \\
\hline 305 & & 14 & 4 & 10 & 4 & 3.09 & 0 & - & - & 4.38 & 4 \\
\hline 315 & & - & - & 138 & 0 & 2.6 & 4 & .. & - & 4.2 & 4 \\
\hline 328 & & 23 & 0 & 2.9 & 0 & 2.5 & 3 & 11 & 0 & 4.3 & 4 \\
\hline 330 & & 14.8 & 3 & $<50$ & NR & 2.54 & 4 & -- & -- & 4.23 & 4 \\
\hline 356 & & 14.7 & 4 & - & -- & 2.71 & 3 & - & - & 4.65 & 1 \\
\hline 370 & & $<20.0$ & NA & $<50.0$ & NR & 3.09 & 0 & -- & -- & 4.25 & 4 \\
\hline 372 & & 14 & 4 & 11 & 4 & 1.88 & 0 & - & - & 4.09 & 3 \\
\hline
\end{tabular}


Table 5. Laboratory performance ratings for standard reference sampie T-169 (trace constituents) -- continued

[MPV, most probable value; Lab, laboratory identification number; OLR, overall laboratory rating for all rated analyses; $\mu \mathrm{g} / \mathrm{L}$, micrograms per liter; $\mathrm{mg} / \mathrm{L}$, milligrams per liter; V/28, number of rated analyses out of 28 possible; RV, reported value; <, less than; NR, not rated; --, not reported.]

\begin{tabular}{|lcll|}
\hline Rating & Absolute Z-value & Rating & Absolute Z-value \\
4 (Excellent) & $0.00-0.50$ & 1 (Marginal) & $1.51-2.00$ \\
3 (Good) & $0.51-1.00$ & 0 (Unsatisfactory) & greater than 2.00 \\
2 (Satisfactory) & $1.01-1.50$ & NR (Not Rated) & \\
\hline
\end{tabular}

\begin{tabular}{|c|c|c|c|c|c|c|c|c|c|c|c|}
\hline \multirow[b]{2}{*}{ Lab } & $\begin{array}{r}\text { Analyte }= \\
M P V= \\
\text { F-pseudosigma }=\end{array}$ & \multicolumn{2}{|c|}{$\begin{array}{l}\text { Manganese } \\
27.0 \mu g / \mathrm{L} \\
0.9\end{array}$} & \multicolumn{2}{|c|}{$\begin{array}{c}\text { Molybdenum } \\
70.6 \mu \mathrm{g} / \mathrm{L} \\
1.7\end{array}$} & \multicolumn{2}{|c|}{$\begin{array}{l}\text { Sodium } \\
10.6 \mathrm{mg} / \mathrm{L} \\
0.3\end{array}$} & \multicolumn{2}{|c|}{$\begin{array}{c}\text { Nickel } \\
10.3 \mu \mathrm{g} / \mathrm{L} \\
0.7\end{array}$} & \multicolumn{2}{|c|}{$\begin{array}{l}\text { Lead } \\
11.4 \mu g / L \\
0.7\end{array}$} \\
\hline & & RV & Rating & $\mathrm{RV}$ & Rating & RV & Rating & RV & Rating & AV & Fating \\
\hline 1 & & 27.1 & 4 & 70.31 & 4 & 10.16 & 3 & 10.57 & 4 & 11.4 & 4 \\
\hline 5 & & 25.9 & 3 & 74.6 & 2 & 10.8 & 4 & 14.6 & 0 & 10 & 0 \\
\hline 7 & & 26.3 & 3 & 65.5 & 2 & 10.5 & 4 & 1.9 & 0 & 11.3 & 4 \\
\hline 8 & & 22.8 & 0 & 70.1 & 4 & 10.6 & 4 & 10.6 & 4 & 12.5 & 1 \\
\hline 10 & & 28 & 3. & -- & -- & - & $\ldots$ & -- & -- & 11.5 & 4 \\
\hline 12 & & $\ldots$ & - & -- & 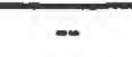 & 12 & 0 & $=$ & - & 13 & 0 \\
\hline 16 & & 27 & 4 & 70 & 4 & 10 & 2 & 10 & 4 & 11 & 3 \\
\hline 21 & & - & - & -- & -- & - & - & -- & -. & - & - \\
\hline 23 & & 26.69 & 4 & 74.4 & 2 & 10.5 & 4 & 10.14 & 4 & 10.23 & 1 \\
\hline 24 & & 26.3 & 3 & 70.5 & 4 & 10.5 & 4 & -- & -- & - & - \\
\hline 25 & & 25 & 2 & -. & - & 9.92 & 2 & 12 & 0 & 11.5 & 4 \\
\hline 26 & & 27.5 & 4 & 70.7 & 4 & 10.7 & 4 & -- & -. & 11.2 & 4 \\
\hline 32 & & 28.4 & 2 & 74.8 & 2 & 9.82 & 2 & 10.9 & 3 & 10.4 & 2 \\
\hline 33 & & 42 & 0 & -- & -- & 10.2 & 3 & .- & - & -. & - \\
\hline 42 & & 25.6 & 2 & 68.5 & 3 & 9.47 & 0 & 10.6 & 4 & 11.3 & 4 \\
\hline 45 & & 27 & 4 & 62.6 & 0 & 10.7 & 4 & 10 & 4 & 9.47 & 0 \\
\hline 46 & & 27 & 4 & -. & -. & 10.8 & 3 & -- & -- & 11.8 & 3 \\
\hline 59 & & 26.8 & 4 & 69.8 & 4 & 10.6 & 4 & 10 & 4 & 12.2 & 2 \\
\hline 64 & & - & - & - & -- & 10.55 & 4. & -. & -- & -- & - \\
\hline 70 & & $26: 4$ & 4 & 73.8 & 3 & 11.1 & 3 & 12.2 & 0 & 9.84 & 0 \\
\hline 76 & & - & - & 70.58 & 4 & 10.97 & 3 & 10.45 & 4 & 11.68 & 4 \\
\hline 86 & & 26.2 & 3 & 67.1 & 3 & 10,8 & 4 & 9.35 & 2 & 11.2 & 4 \\
\hline 89 & & 24.8 & 1 & -- & -. & 11.1 & 3 & 10.9 & 3 & 11.6 & 4 \\
\hline 97 & & 28 & 3 & 65.9 & 2 & 10.2 & 3 & 12.6 & 0 & 12.5 & 1 \\
\hline 105 & & 27 & 4 & 74.65 & 2 & 10.34 & 4 & $<50$ & NR & 10.96 & 3 \\
\hline 109 & & $<50$ & NR & - & $\overline{-}$ & 10.51 & 4 & -- &.- & 12.02 & 3 \\
\hline 110 & & - & 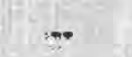 & -- & -- & 10.092 & 3 & .. & -- & -. & - \\
\hline 113 & & 26.8 & 4 & 69.9 & 4 & 10.5 & 4 & 10.1 & 4 & 11,8 & 3 \\
\hline 121 & & 27 & 4 & -. & -- & 10.7 & 4 & -- & -- & 10.8 & 3 \\
\hline 134 & & 27.36 & 4 & 70.46 & 4 & 10.84 & 4 & 10.07 & 4 & 11.7 & 4 \\
\hline 138 & & 27 & 4 & 71.2 & 4 & 10.6 & 4 & 10.6 & 4 & 11.1 & 4 \\
\hline 142 & & 26. & 3 & 71.3 & 4 & 10.4 & 4 & 10.3 & 4 & 10.7 & 2 \\
\hline 146 & & 26.6 & 4 & 68.5 & 3 & 10.8 & 4 & 10.1 & 4 & 12 & a \\
\hline 147 & & - & - & -. & -- & -1 & - & -. & -- & 11.3 & 4 \\
\hline 149 & & 26.6 & 4 & 71 & 4 & 11,2 & 2 & 10.3 & 4 & 11.5 & 4 \\
\hline 180 & & 24.8 & T & 72.3 & 4 & 10.7 & 4 & 6.83 & 0 & 9.88 & 0 \\
\hline 183 & & - & - & .. & -- & -. & - & 23.2 & 0 & 15.1 & 0 \\
\hline 190 & & 26.9 & 4 & - & -- & - & - & 10.6 & 4 & 10.7 & 2 \\
\hline 193 & & -. & -. & -- & -. & 10.8 & 3 & $<12.5$ & NR & 13 & 0 \\
\hline 198 & & 24.3 & 1 & 71.2 & 4 & 10.6 & 4 & 9.93 & 3 & 10.9 & 3 \\
\hline 212 & & 27.5 & 4 & 69.4 & 4 & 10.9 & 3 & 8.6 & 0 & 13.9 & 0 \\
\hline 220 & & 25.74 & 3 & -- & -- & 10.566 & 4 & -- & -- & - & - \\
\hline 227 & & - & - & -- & -- & - & - & .- & -- & 11.3 & 4 \\
\hline 234 & & 27.5 & 4 & 71.3 & 4 & 10.8 & 4 & 10.3 & 4 & 12.4 & 2 \\
\hline 245 & & 27.93 & 3 & 70.79 & 4 & - & -. & 11.08 & 2 & 12.22 & 2 \\
\hline
\end{tabular}


Table 5. Laboratory performance ratings for standard reference sample T-169 (trace const/tuents) -- continued [MPV, most probable value; Lab, laboratory identification number; OLR, overail laboratory rating for all rated analyses; $\mu \mathrm{g} / \mathrm{L}$, micrograms per liter; mg/L, milligrams per liter; V/28, number of rated analyses out of 28 possible; RV, reported value; <, less than; NR, not rated; --, not reported.]

\begin{tabular}{|lcll|}
\hline Rating & Absolute Z-value & Rating & Absolute Z-value \\
4 (Excellent) & $0.00-0.50$ & 1 (Marginal) & $1.51-2.00$ \\
3 (Good) & $0.51-1.00$ & 0 (Unsatisfactory) & greater than 2.00 \\
2 (Satisfactory) & $1.01-1.50$ & NR (Not Rated) & \\
\hline
\end{tabular}

\begin{tabular}{|c|c|c|c|c|c|c|c|c|c|c|c|}
\hline \multirow[b]{2}{*}{ Lab } & \multirow{2}{*}{$\begin{array}{r}\text { Analyte }= \\
\text { MPV }= \\
\text { F-pseudosigma }= \\
\end{array}$} & \multicolumn{2}{|c|}{$\begin{array}{c}\text { Manganese } \\
27.0 \mu \mathrm{g} / \mathrm{L} \\
0.9\end{array}$} & \multicolumn{2}{|c|}{$\begin{array}{c}\text { Molybdenum } \\
70.6 \mu \mathrm{g} / \mathrm{L} \\
1.7 \\
\end{array}$} & \multicolumn{2}{|c|}{$\begin{array}{l}\text { Sodlum } \\
10.6 \mathrm{mg} / \mathrm{L} \\
0.3\end{array}$} & \multicolumn{2}{|c|}{$\begin{array}{l}\text { Nickel } \\
10.3 \mu \mathrm{g} / \mathrm{L} \\
0.7\end{array}$} & \multicolumn{2}{|c|}{$\begin{array}{l}\text { Lead } \\
11.4 \mu \mathrm{g} / \mathrm{L} \\
0.7\end{array}$} \\
\hline & & RV & Rating & $\mathrm{RV}$ & Rating & RV & Rating & RV & Rating & $\mathrm{RV}$ & Rating \\
\hline 247 & & 25.3 & 2 & 71.2 & 4 & 9.8 & 2 & 10.4 & 4 & 11.3 & 4 \\
\hline 255 & & 26.8 & 4 & -- & -- & - & - & 11.4 & 1 & 12.2 & 2 \\
\hline 258 & & 27.75 & 3 & 65.1 & 1 & - & - & 10.2 & 4 & 11.03 & 3 \\
\hline 259 & & 27 & 4 & 71 & 4 & 10.9 & 3 & 10 & 4 & 11.5 & 4 \\
\hline 265 & & 26.5 & 4 & 71.5 & 4 & 10.5 & 4 & 10 & 4 & 11 & 3 \\
\hline 279 & & - & 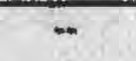 & -. & -. & 11.23 & 2 & - & - & - & - \\
\hline 304 & & 27.6 & 4 & 71 & 4 & - & $\ldots$ & 10.7 & 3 & 11.5 & 4 \\
\hline 305 & & 27 & 4 & 61.7 & 0 & 10.9 & 3 & 10.3 & 4 & 12 & 3 \\
\hline 315 & & 42 & 0 & -. & -- & 10.6 & 4 & -- & - & - & - \\
\hline 328 & & 30 & 0 & 71 & 4 & 11 & 3 & 9.9 & 3 & 11 & 3 \\
\hline 330 & & 29.1 & 1 & 70.5 & 4 & 10.7 & 4 & 12.9 & 0 & 11.8 & 3 \\
\hline 356 & & 27.3 & 4 & 71.1 & 4 & 11.1 & 3 & 10.4 & 4 & 5.74 & 0 \\
\hline 370 & & 28.1 & 3 & 64 & 1 & 11.9 & 0 & 9.89 & 3 & 11.8 & 3 \\
\hline 372 & & 27 & 4 & 61 & 0 & 8.56 & 0 & 11 & 2 & 9 & 0 \\
\hline
\end{tabular}


Table 5. Laboratory performance ratings for standard reference sample T-169 (trace constituents) -- continued [MPV, most probable value; Lab, laboratory identification number; OLR, overall laboratory rating for all rated analyses; $\mu \mathrm{g} / \mathrm{L}$, micrograms per liter; $\mathrm{mg} / \mathrm{L}$, milligrams per liter; V/28, number of rated analyses out of 28 possible; RV, reported value; <, less than; NR, not rated; --, not reported.]

\begin{tabular}{|lcll|}
\hline Rating & Absolute Z-value & Rating & Absolute Z-value \\
4 (Excellent) & $0.00-0.50$ & 1 (Marginal) & $1.51-2.00$ \\
3 (Good) & $0.51-1.00$ & 0 (Unsatisfactory) & greater than 2.00 \\
2 (Satisfactory) & $1.01-1.50$ & NR (Not Rated) & \\
\hline
\end{tabular}

\begin{tabular}{|c|c|c|c|c|c|c|c|c|c|c|c|}
\hline \multirow[b]{2}{*}{ Lab } & \multirow{2}{*}{$\begin{array}{r}\text { Analyte }= \\
\text { MPV }= \\
\text { F-pseudosigma }= \\
\end{array}$} & \multicolumn{2}{|c|}{$\begin{array}{l}\text { Antimony } \\
3.33 \mu \mathrm{g} / \mathrm{L} \\
0.44 \\
\end{array}$} & \multicolumn{2}{|c|}{$\begin{array}{l}\text { Selenlum } \\
2.94 \mu \mathrm{g} / \mathrm{L} \\
0.37\end{array}$} & \multicolumn{2}{|c|}{$\begin{array}{l}\text { Sillea } \\
6.04 \mathrm{mg} / \mathrm{L} \\
0.23\end{array}$} & \multicolumn{2}{|c|}{$\begin{array}{c}\text { Strontlum } \\
174 \mu \mathrm{g} / \mathrm{L} \\
6\end{array}$} & \multicolumn{2}{|c|}{$\begin{array}{l}\text { Thallium } \\
4.80 \mu \mathrm{g} / \mathrm{L} \\
0.51\end{array}$} \\
\hline & & RV & Rating & RV & Rating & RV & Rating & RV & Rating & RV & Pating \\
\hline 1 & & 3.71 & 3 & 2.83 & 4 & 6.1 & 4 & 171.7 & 4 & 4.8 & 4 \\
\hline 5 & & 4.48 & 0 & 2.7 & 3 & 2.9 & 0 & 174 & 4 & 10.3 & 0 \\
\hline 7 & & 3.4 & 4 & 2.9 & 4 & 5.87 & 3 & 175 & 4 & 4.8 & 3. \\
\hline 8 & & 3.2 & 4 & 2.6 & 3 & 6.4 & 2 & 186 & 2 & 4.7 & 4 \\
\hline 10 & & - & - & 2.5 & 2 & - & - & -- & -- & - & - \\
\hline 12 & & - & $\cdots$ & 2 & 0 & - & - & - & - & -- & - \\
\hline 16 & & 3.4 & 4 & 2.6 & 3 & - & - & 169 & 3 & 5.1 & 3 \\
\hline 21 & & - & - & - & - & - & - & - & -- & - & - \\
\hline 23 & & - & - & 17.1 & 0 & - & - & -- & - & 5.26 & 3 \\
\hline 24 & & - & - & - & - & 6.21 & 3 & 177 & 4 & - & - \\
\hline 25 & & $<50$ & NA & $<16$ & NR & 4.18 & 0 & 173 & 4 & $<10$ & NR \\
\hline 26 & & - & - & - & - & - & - & - & - & - & - \\
\hline 32 & & 3.1 & 3 & 3.1 & 4 & 6.1 & 4 & 183 & 2 & 4.7 & 4 \\
\hline 33 & & - & - & -- & - & 2.46 & 0 & 161.4 & 2 & - & - \\
\hline 42 & & 3.13 & 4 & 2.85 & 4 & 5.59 & 2 & 192 & 0 & 4.6 & 4 \\
\hline 45 & & 2.85 & 2 & 2.94 & 4 & - & - & -- & - & 3.94 & 1 \\
\hline 46 & & - & - & - & - & - & - & - & - & - & - \\
\hline 59 & & $<5$ & NA & $<5$ & NR & - & $\cdots$ & 173 & 4 & $<10$ & NR \\
\hline 64 & & - & - & - & - & 5.8 & 3 & -- & - & - & - \\
\hline 70 & & 7.43 & 0 & $<10$ & NR & 6.26 & 3 & - & - & 4.67 & 4 \\
\hline 76 & & . & - & 2.987 & 4 & $\mu$ & - & 175.1 & 4 & 4.903 & 4 \\
\hline 66 & & - & - & -- & -- & - & - & 174 & 4 & - & - \\
\hline 89 & & $<5: 00$ & NR & 2 & 0 & - & - & -- & -- & 4.9 & 4 \\
\hline 97 & & 3.32 & 4 & 2.89 & 4 & 6.59 & 1 & 170 & 4 & 5.72 & 1 \\
\hline 105 & & 3.13 & 4 & $<7$ & NR & 6.056 & 4 & 174 & 4 & 4.84 & 4 \\
\hline 109 & & - & - & - & -- & - & - & 158.9 & 1 & + & - \\
\hline 110 & & - & $\ldots$ & -- & -- & 6.0455 & 4 & -- & -- & - & - \\
\hline 113 & & - & - & 2.45 & 2 & - & -. & 172.6 & 4 & - & - \\
\hline 121 & & - & $\therefore$ & 3.2 & 3 & 6 & 4 & 177 & 4 & 4.2 & 2 \\
\hline 134 & & 3.2 & 4 & 2.95 & 4 & 6.04 & 4 & 172.55 & 4 & 5.2 & 3 \\
\hline 138 & & 3 & 3 & 3.26 & 3 & . & - & 172 & 4 & 5 & 4 \\
\hline 142 & & 3.42 & 4 & 3.06 & 4 & 6.04 & 4 & 179 & 3 & 4.8 & 4 \\
\hline 146 & & $<20.0$ & NR & $<10.0$ & NR & - & - & -- & $\cdots$ & 7.26 & 0 \\
\hline 147 & & - & - & -- & - & - & $\ldots$ & 174 & 4 & - & - \\
\hline 149 & & 3.2 & 4 & 3.1 & 4 & - & - & - & - & 4.5 & 3 \\
\hline 180 & & 3.32 & 4 & 2.79 & 4 & - & - & - & - & 4.13 & 2 \\
\hline 183 & & 4.22 & 0 & $<5.0$ & NR & - & - & - & - & 5.62 & 1 \\
\hline 190 & & - & - & 2.2 & 1 & 6.1 & 4 & - & - & - & $\rightarrow$ \\
\hline 193 & & $<10$ & NR & $<5$ & NR & - & $\cdots$ & .. & - & - & - \\
\hline 198 & & 3.68 & 3 & 3.36 & 2 & - & - & -- & -- & 4.7 & 4 \\
\hline 212 & & 3.8 & 2 & $<15$ & NR & 6.1 & 4 & 182 & 3 & $<10$ & NR \\
\hline 220 & & - & - & 1.8 & 0 & 6.11 & 4 & - & -- & - & - \\
\hline 227 & & - & - & -- & - & - & - & -- & - & - & - \\
\hline 234 & & 2.79 & 2 & 2.2 & 1 & 5.97 & 4 & 177 & 4 & 4.96 & 4 \\
\hline 245 & & 3.328 & 4 & 3 & 4 & - & - & 180.3 & 3 & 5.182 & 3 \\
\hline
\end{tabular}


Table 5. Laboratory performance ratings for standard reference sample T-169 (trace constituents) -- continued [MPV, most probable value; Lab, laboratory identification number; OLR, overall laboratory rating for all rated analyses; $\mu \mathrm{g} / \mathrm{L}$, micrograms per liter; $\mathrm{mg} / \mathrm{L}$, milligrams per liter; V/28, number of rated analyses out of 28 possible; RV, reported value; <, less than; NR, not rated; --, not reported.]

\begin{tabular}{|llll|}
\hline Rating & Absolute Z-value & Rating & Absolute Z-value \\
4 (Excellent) & $0.00-0.50$ & 1 (Marginal) & $1.51-2.00$ \\
3 (Good) & $0.51-1.00$ & 0 (Unsatisfactory) & greater than 2.00 \\
2 (Satisfactory) & $1.01-1.50$ & NR (Not Rated) & \\
\hline
\end{tabular}

\begin{tabular}{|c|c|c|c|c|c|c|c|c|c|c|}
\hline \multirow[t]{2}{*}{$\begin{array}{r}\text { Analyte }= \\
M P V= \\
\text { F-pseudosigma }=\end{array}$} & \multicolumn{2}{|c|}{$\begin{array}{l}\text { Antimony } \\
3.33 \mu g / 2 \\
0.44\end{array}$} & \multicolumn{2}{|c|}{$\begin{array}{l}\text { Selenlum } \\
2.94 \mu \mathrm{g} / \mathrm{L} \\
0.37\end{array}$} & \multicolumn{2}{|c|}{\begin{tabular}{l}
\multicolumn{1}{c}{ Sillca } \\
$6.04 \mathrm{mg} / \mathrm{L}$ \\
0.23
\end{tabular}} & \multicolumn{2}{|c|}{$\begin{array}{l}\text { Strontium } \\
174 \mu \mathrm{g} / \mathrm{L} \\
6\end{array}$} & \multicolumn{2}{|c|}{$\begin{array}{l}\text { Thallium } \\
4.80 \mu \mathrm{g} / \\
0.51\end{array}$} \\
\hline & AV & Rating & RV & Rating & PV & Rating & $\mathrm{RV}$ & Rating & RV & Rating \\
\hline 247 & 2.34 & 0 & - & $=$ & - & $\ldots$ & 150 & 0 & 4.77 & 4 \\
\hline 255 & . & - & 3.05 & 4 & - & - & - & - & -5 & - \\
\hline 256 & 2.78 & 2 & 0.56 & 0 & 5.3 & 0 & 186 & 2 & - & - \\
\hline 259 & - & - & - & - & 5.97 & 4 & 180 & 3 & -. & - \\
\hline 265 & 3.2 & 4 & 3 & 4 & 5.8 & 3 & 172 & 4 & 4.5 & 3 \\
\hline 279 & - & - & - & - & 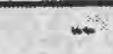 & - & - & - & - & $\rightarrow$ \\
\hline 304 & 3.33 & 4 & - & -- & 4 & -. & 177 & 4 & - & - \\
\hline 305 & 9 & 0 & $<5$ & NR & - & - & -- & -- & 4.2 & 2 \\
\hline 315 & - & - & -. & - & - & - & -- & - & - & - \\
\hline 328 & 5.3 & 0 & 3.1 & 4 & 6.3 & 3 & 150 & 0 & 4.5 & 3 \\
\hline 330 & 3.5 & 4 & 3.3 & 3 & - & - & 183 & 2 & 4.9 & 4 \\
\hline 356 & 3.61 & 3 & 3.14 & 3 & 6.33 & 3 & 203 & 0 & 12.8 & 0 \\
\hline 370 & 5.72 & 0 & $<5.00$ & NR & 3 & 0 & - & - & 3.62 & 0 \\
\hline 372 & 4 & 1 & 3 & 4 & 6.11 & 4 & 162 & 2 & 6 & 0 \\
\hline
\end{tabular}


Table 5. Laboratory performance ratings for standard reference sample T-169 (trace constituents) -- continued [MPV, most probable value; Lab, laboratory identification number; OLR, overall laboratory rating for all rated analyses; $\mu \mathrm{g} / \mathrm{L}$, micrograms per liter; $\mathrm{mg} / \mathrm{L}$, milligrams per liter; V/28, number of rated analyses out of 28 possible; RV, reported value; <, less than; NR, not rated; --, not reported.]

\begin{tabular}{|llll|}
\hline Rating & Absolute Z-value & Rating & Absolute Z-value \\
4 (Excellent) & $0.00-0.50$ & 1 (Marginal) & $1.51-2.00$ \\
3 (Good) & $0.51-1.00$ & 0 (Unsatisfactory) & greater than 2.00 \\
2 (Satisfactory) & $1.01-1.50$ & NR (Not Rated) & \\
\hline
\end{tabular}

\begin{tabular}{|c|c|c|c|c|c|c|c|}
\hline \multirow[b]{2}{*}{ Lab } & $\begin{array}{r}\text { Analyte }= \\
M P V= \\
\text { F-pseudosigma }=\end{array}$ & \multicolumn{2}{|c|}{$\begin{array}{l}\text { Uranium } \\
1.71 \mu g / \mathrm{L} \\
0.11\end{array}$} & \multicolumn{2}{|c|}{$\begin{array}{l}\text { Vanadium } \\
7.42 \mu \mathrm{g} / \mathrm{L} \\
0.38\end{array}$} & \multicolumn{2}{|c|}{$\begin{array}{c}\text { Zlne } \\
19.2 \mu g / \mathrm{L} \\
1.1\end{array}$} \\
\hline & & AV & Pating & $\mathrm{RV}$ & Rating & RV & Rating \\
\hline 1 & & 1.73 & 4 & 7.59 & 4 & 19.6 & 4 \\
\hline 5 & & - & - & 7.1 & 3 & 18.4 & 3 \\
\hline 7 & & 1.6 & 3 & 7.8 & 3 & 19.9 & 3 \\
\hline 8 & & $1: 6$ & 3 & 7.6 & 4 & 24.2 & 0 \\
\hline 10 & & - & - & -. & - & 18 & 2 \\
\hline 12 & & 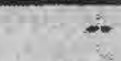 & - & -- & - & - & - \\
\hline 16 & & 1.9 & 1 & 7 & 2 & 19 & 4 \\
\hline 21 & & - & - & - & - & - & - \\
\hline 23 & & - & $\ldots$ & - & - & 20.98 & 1 \\
\hline 24 & & $=$ & $=$ & -. & - & 19.6 & 4 \\
\hline 25 & & 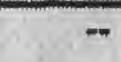 & 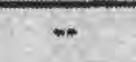 & $<19$ & NR & 14 & 0 \\
\hline 26 & & - & - & -. & -- & 18.1 & 2 \\
\hline 32 & & 1.79 & 3 & 7.7 & 3 & 16.6 & 0 \\
\hline 33 & & - & - & -- & -- & - & $\cdots$ \\
\hline 42 & & 1.75 & 4 & 7.24 & 4 & 18.7 & 4 \\
\hline 45 & & 1.34 & 0 & 7.14 & 3 & 19.2 & 4 \\
\hline 46 & & - & - & -- & -. & - & - \\
\hline 59 & & - & - & 7.21 & 3 & 19.9 & 3 \\
\hline 64 & & $-\cdots$ & - & -- & -- & - & - \\
\hline 70 & & $<10.0$ & NR & 7.56 & 4 & 19.7 & 4 \\
\hline 76 & & - & - & - & - & - & - \\
\hline 86 & & - & - & 7.2 & 3 & 18.7 & 4 \\
\hline 89 & & - & - & 10.2 & 0 & 17.3 & 1 \\
\hline 97 & & - & - & $<7.7$ & NR & 12.2 & 0 \\
\hline 105 & & - & - & $<20$ & NR & $<10.0$ & 0 \\
\hline 109 & & - & - & $=$ & $=$ & - & - \\
\hline 110 & & - & - & -- & -. & - & - \\
\hline 113 & & $\%$ & - & -- & -. & 19.3 & 4 \\
\hline 121 & & - & - & 7.8 & 3 & 18 & 2 \\
\hline 134 & & - & - & 7.48 & 4 & 19.2 & 4 \\
\hline 138 & & - & - & 7.39 & 4 & 20 & 3 \\
\hline 142 & & 1.72 & 4 & 7.42 & 4 & 19 & 4 \\
\hline 146 & & - & - & 7.95 & 2 & 18.7 & 4 \\
\hline 147 & & 1.68 & 4 & -- & - & 18.2 & 3 \\
\hline 149 & & 1.6 & 3. & 7.2 & 3 & 19.4 & 4 \\
\hline 180 & & - & - & 6.84 & 1 & 18.7 & 4 \\
\hline 183 & & - & $\omega$ & 10.09 & 0 & - & - \\
\hline 190 & & - & - & - & -. & 18.8 & 4 \\
\hline 193 & & - & - & - & - & $<25$ & NR \\
\hline 198 & & $=$ & - & 7.23 & 4 & 20.3 & 2 \\
\hline 212 & & - & - & 9 & 0 & 32 & 0 \\
\hline 220 & & -. & - & -- & -- & - & - \\
\hline 227 & & - & - & -- & -. & 19 & 4 \\
\hline 234 & & - & - & 7.67 & 3 & 21.5 & 0 \\
\hline 245 & & 1.72 & 4 & 7.39 & 4 & 19.83 & 3 \\
\hline
\end{tabular}


Table 5. Laboratory performance ratings for standard reference sample T-169 (trace constituents) - continued [MPV, most probable value; Lab, laboratory identification number; OLR, overall laboratory rating for all rated analyses; $\mu \mathrm{g} / \mathrm{L}$, micrograms per liter; $\mathrm{mg} / \mathrm{L}$, milligrams per liter; V/28, number of rated analyses out of 28 possible; RV, reported value; <, less than; NR, not rated; --, not reported.]

\begin{tabular}{|llll|}
\hline Rating & Absolute Z-value & Rating & Absolute Z-value \\
4 (Excelient) & $0.00-0.50$ & 1 (Marginal) & $1.51-2.00$ \\
3 (Good) & $0.51-1.00$ & 0 (Unsatisfactory) & greater than 2.00 \\
2 (Satisfactory) & $1.01-1.50$ & NR (Not Rated) & \\
\hline
\end{tabular}

\begin{tabular}{|c|c|c|c|c|c|c|c|}
\hline \multirow[b]{2}{*}{ Lab } & $\begin{array}{r}\text { Analyte }= \\
M P V= \\
\text { F-pseudosigma }=\end{array}$ & \multicolumn{2}{|c|}{$\begin{array}{l}\text { Uranlum } \\
1.71 \mu g h \\
0.11\end{array}$} & \multicolumn{2}{|c|}{$\begin{array}{l}\text { Vanadlum } \\
7.42 \mu \mathrm{g} / \mathrm{L} \\
0.38\end{array}$} & \multicolumn{2}{|c|}{$\begin{array}{c}\text { Zinc } \\
19.2 \mu \mathrm{g} / \mathrm{L} \\
1.1\end{array}$} \\
\hline & & RV & Rating & RV & Rating & RV & Rating \\
\hline 247 & & $\pi$ & - & 7.19 & 3 & 18.3 & 3 \\
\hline 255 & & - & - & -- & -. & 19.3 & 4 \\
\hline 256 & & - & 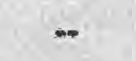 & 7.66 & 3 & 17.75 & 2 \\
\hline 259 & & - & - & -- & -. & 18.6 & 3 \\
\hline 265 & & 1.7 & 4 & 7.5 & 4 & 20 & 3 \\
\hline 279 & & - & - & - & $\overline{-}$ & - & $=$ \\
\hline 304 & & - & - & - & - & 19.3 & 4 \\
\hline 305 & & $\rightarrow$ & - & 7.1 & 3 & 20.1 & 3 \\
\hline 315 & & - & - & - & -- & - & - \\
\hline 328 & & 1.7 & 4 & 1.5 & 0 & 23 & 0 \\
\hline 330 & & 1.9 & 1 & 8.1 & 1 & 21 & 1 \\
\hline 356 & & - & - & 6.83 & 1 & 18.8 & 4 \\
\hline 370 & & - & - & 19 & 0 & $<30.0$ & NR \\
\hline 372 & & + & - & 6 & 0 & 20 & 3 \\
\hline
\end{tabular}


Table 6. Laboratory performance ratings for standard reference sample $M-162$ (major constituents)

[MPV, most probable value; Lab, laboratory identification number; OLR, overall laboratory rating for all rated analyses; $\mathrm{mg} / \mathrm{L}$, milligrams per liter; $\mu \mathrm{g} / \mathrm{L}$, micrograms per liter; $\mu \mathrm{S} / \mathrm{cm}$, microsiemens per centimeter at 25 degrees Celsius; V/16, number of rated analyses out of 16 possible; RV, reported value; <, less than; NR, not rated; --, not reported.]

\begin{tabular}{|lcll|}
\hline Rating & Absolute Z-value & Rating & Absolute Z-value \\
4 (Excellent) & $0.00-0.50$ & 1 (Marginal) & $1.51-2.00$ \\
3 (Good) & $0.51-1.00$ & 0 (Unsatisfactory) & greater than 2.00 \\
2 (Satisfactory) & $1.01-1.50$ & NR (Not Rated) & \\
\hline
\end{tabular}

\begin{tabular}{|c|c|c|c|c|c|c|c|c|c|c|c|c|}
\hline \multirow[b]{2}{*}{ Lab } & \multicolumn{2}{|c|}{$\begin{array}{r}\text { Analyte }= \\
M P V= \\
\text { F-pseudosigma }=\end{array}$} & \multicolumn{2}{|c|}{$\begin{array}{l}\text { Alkalinity } \\
11.0 \mathrm{mg} / \\
1.7\end{array}$} & \multicolumn{2}{|c|}{$\begin{array}{l}\text { Boron } \\
57.3 \mu \mathrm{g} / \mathrm{L} \\
6.2 \\
\end{array}$} & \multicolumn{2}{|c|}{$\begin{array}{l}\text { Calclum } \\
15.8 \mathrm{mg} / \mathrm{L} \\
0.5\end{array}$} & \multicolumn{2}{|c|}{$\begin{array}{l}\text { Chloride } \\
26.5 \mathrm{mg} / \mathrm{L} \\
1.0\end{array}$} & \multicolumn{2}{|c|}{$\begin{array}{l}\text { Fluoride } \\
1.05 \mathrm{mg} / \mathrm{L} \\
0.09\end{array}$} \\
\hline & OLR & $V / 16$ & AV & Rating & $\mathrm{RV}$ & Rating & RV & Rating & RV & Rating & $\mathrm{RV}$ & Rating \\
\hline 1 & 3.8 & 14 & 11.28 & 4 & - & -- & 16.44 & 3 & 26.31 & 4 & 1.06 & 4 \\
\hline 4 & 0.3 & 3 & 22 & 0 & -- & -- & 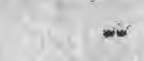 & - & 28.8 & 1 & - & - \\
\hline 5 & 1.7 & 16 & 10.7 & 4 & $<10.0$ & 0 & 6.26 & 0 & 26.51 & 4 & 0.99 & 3 \\
\hline 8 & 2.3 & 16 & 13 & 2 & 45 & 1 & 16.3 & 3 & 25.9 & 4 & 0.88 & 1 \\
\hline 10 & 3.7 & 12 & 10.7 & 4 & - & - & 15.8 & 4 & 26.8 & 4 & 1.14 & 2 \\
\hline 12 & 1.9 & 10 & 11 & 4 & - & - & 16 & 4 & 25 & 2 & - & - \\
\hline 16 & 2.4 & 15 & 10 & 3 & 47 & 1 & 16 & 4 & 21.7 & 0 & 1.03 & 4 \\
\hline 23 & 2.9 & 11 & 12 & 3 & 0.21 & 0 & 15.52 & 4 & -- & - & 1.08 & 4 \\
\hline 24 & 3.5 & 13 & 11 & 4 & 56.7 & 4 & 16 & 4 & 26.8 & 4 & 1.06 & 4 \\
\hline 26 & 1.1 & 14 & 16 & 0 & 50 & 2 & 13 & 0 & 24.9 & 2 & 0.04 & 0 \\
\hline 26 & 3.2 & 9 & - & $\cdots$ & - & - & 16.3 & $\overline{3}$ & 26.6 & 4 & 0.88 & 1 \\
\hline 32 & 3.2 & 16 & 10.8 & 4 & 57 & 4 & 15.4 & 4 & 25.9 & 4 & 1.03 & 4 \\
\hline 33 & 2.1 & 12 & 9.1 & 2 & -- & - & 16.1 & 4 & 24.98 & 2 & 1.17 & 2 \\
\hline 38 & 3.2 & 9 & 10.61 & 4 & -- & -- & 17.6 & 0 & -- & - & - & - \\
\hline 42 & 2.5 & 15 & 14.1 & 1 & 54.3 & 4 & 15.5 & 4 & 27.4 & 3 & 1.05 & 4 \\
\hline 45 & 3.8 & 12 & 11.4 & 4 & 55.8 & 4 & 15.7 & 4 & 27.5 & 3 & 0.97 & 3 \\
\hline 46 & 3.8 & 12 & 11.4 & 4 & -- & -- & 16 & 4 & 26.7 & 4 & 1.1 & 3 \\
\hline 59 & 3.0 & 14 & 8.77 & 2 & 50.7 & 2 & 16.2 & 4 & 24.7 & 2 & 1.13 & 3 \\
\hline 64 & 3.1 & 10 & - & - & . & -. & 16.5 & 3 & 27.3 & 3 & $*$ & + \\
\hline 70 & 3.6 & 13 & 11 & 4 & $<100$ & NR & 16.2 & 4 & 27.9 & 2 & 1.05 & 4 \\
\hline 76 & 4.0 & 5 & - & $\omega$ & - & -- & 16.04 & 4 & - & - & + & - \\
\hline 85 & 2.4 & 16 & 12.8 & 2 & 37 & 0 & 16.6 & 2 & 26.5 & 4 & 1.05 & 4 \\
\hline 86 & 3.2 & 13 & - & - & 58.1 & 4 & 16 & 4 & 27.2 & 3 & 1.1 & 3 \\
\hline 89 & 3.3 & 14 & 10.4 & 4 & -- & - & 15.7 & 4 & 26.3 & 4 & 1.06 & 4 \\
\hline 97 & 3.0 & 12 & 10 & 3 & -. & - & 16.1 & 4 & 27.7 & 3 & 1.11 & 3 \\
\hline 102 & 2.0 & 12 & - & - & - & -- & 15.9 & 4 & 28.2 & 2 & $<0.5$ & 0 \\
\hline 105 & 3.0 & 14 & 11.8 & 4 & $<200$ & NR & 15.42 & 4 & 27 & 4 & 1.16 & 2 \\
\hline 109 & 3.3 & 11 & 13.94 & 1 & -- & -- & 15.6 & 4 & 26 & 4 & 1 & 3 \\
\hline 113 & 3.6 & 13 & 9.72 & 3 & - & -- & 15.8 & 4 & 25.7 & 3 & 1.1 & 3 \\
\hline 121 & 3.3 & 6 & - & - & -- & -- & 15.8 & 4 & . & - & -.. & - \\
\hline 134 & 3.8 & 16 & 12 & 3 & 58.63 & 4 & 16.23 & 3 & 25.96 & 4 & 1.02 & 4 \\
\hline 138 & 3.5 & 16 & 9.21 & 2 & 57.5 & 4 & 15.4 & 4 & 28.2 & 2 & 1.05 & 4 \\
\hline 142 & 3.4 & 16 & 11.6 & 4 & 60.7 & 3 & 15.8 & 4 & 27.2 & 3 & 1.05 & 4 \\
\hline 146 & 2.3 & 12 & 16.6 & 0 & -. & - & 15.7 & 4 & 29.3 & 0 & 1.1 & 3 \\
\hline 149 & 3.0 & 7 & 11 & 4 & -- & -- & - & $\ldots$ & 55.6 & 0 & 1.1 & 3 \\
\hline 180 & 2.8 & 13 & 14.6 & 0 & 67.5 & 1 & 16 & 4 & 27.7 & 3 & 0.982 & 3 \\
\hline 183 & 2.3 & 7 & 9 & 2 & -- & - & - & - & 21.55 & 0 & 1.15 & 2 \\
\hline 190 & 3.4 & 14 & 10.8 & 4 & - & -. & 15.5 & 4 & 27.3 & 3 & 1.03 & 4 \\
\hline 193 & 3.7 & 7 & 9.3 & 3 & -- & -- & 16.1 & 4 & -- & - & - & $\therefore$ \\
\hline 208 & 3.5 & 2 & - & - & -- & - & - & - & 26 & 4 & $\therefore$ & - \\
\hline 212 & 2.4 & 14 & 8.9 & 2 & 56.9 & 4 & 14.9 & 2 & 26.1 & 4 & 1.1 & 3 \\
\hline 220 & 3.1 & 8 & 12.334 & 3 & - & - & 16.44 & 3 & 29.82 & 0 & - & - \\
\hline 224 & 2.9 & 13 & 10 & 3 & - & - & 14.842 & 2 & 27.34 & 3 & 1.07 & 4 \\
\hline 227 & 3.3 & 6 & 13.3 & 2 & -. & - & - & - & 25.79 & 3 & - & - \\
\hline 234 & 2.8 & 16 & 12.2 & 3 & 64 & 2 & 16.6 & 2 & 23.1 & 0 & $t .03$ & 4 \\
\hline
\end{tabular}


Table 6. Laboratory performance ratings for standard reference sampie M-162 (major constituents) - continued [MPV, most probable value; Lab, laboratory identification number; OLR, overall laboratory rating for all rated analyses; $\mathrm{mg} / \mathrm{L}$, milligrams per liter; $\mu \mathrm{g} / \mathrm{L}$, micrograms per liter; $\mu \mathrm{S} / \mathrm{cm}$, microsiemens per centimeter at 25 degrees Celsius; $\mathrm{V} / 16$, number of rated analyses out of 16 possible; RV, reported value; <, less than; NR, not rated; --, not reported.]

\begin{tabular}{|lcll|}
\hline Rating & Absolute Z-value & Rating & Absolute Z-value \\
\hline (Excellent) & $0.00-0.50$ & 1 (Marginal) & $1.51-2.00$ \\
(Good) & $0.51-1.00$ & 0 (Unsatisfactory) & greater than 2.00 \\
2 (Satisfactory) & $1.01-1.50$ & NR (Not Rated) & \\
\hline
\end{tabular}

\begin{tabular}{|c|c|c|c|c|c|c|c|c|c|c|c|c|}
\hline \multirow[b]{2}{*}{ Lab } & \multicolumn{2}{|c|}{$\begin{array}{r}\text { Analyte }= \\
\mathrm{MPV}= \\
\text { F-pseudosigma }=\end{array}$} & \multicolumn{2}{|c|}{$\begin{array}{l}\text { Alkalinity } \\
11.0 \mathrm{mg} / \\
1.7\end{array}$} & \multicolumn{2}{|c|}{$\begin{array}{c}\text { Boron } \\
57.3 \mu \mathrm{g} / \mathrm{L} \\
6.2 \\
\end{array}$} & \multicolumn{2}{|c|}{$\begin{array}{l}\text { Caiclum } \\
15.8 \mathrm{mg} / \mathrm{L} \\
0.5\end{array}$} & \multicolumn{2}{|c|}{$\begin{array}{l}\text { Chlorlde } \\
26.5 \mathrm{mg} / \mathrm{L} \\
1.0 \\
\end{array}$} & \multicolumn{2}{|c|}{$\begin{array}{l}\text { Fluoride } \\
1.05 \mathrm{mg} / \mathrm{L} \\
0.09\end{array}$} \\
\hline & OLR & $\mathrm{V} / 16$ & RV & Rating & RV & Rating & $\mathrm{BV}$ & Rating & RV & Rating & RV & Rating \\
\hline 245 & 3.6 & 5 & - & - & -- & $\cdots$ & 15.96 & 4 & -. & -- & $\cdots$ & - \\
\hline 247 & 2.3 & 15 & 11 & 4 & 140 & 0 & 14.4 & 1 & 27 & 4 & 1.06 & 4 \\
\hline 255 & 3.5 & 4 & - & - & .. & - & 15.6 & 4 & -- & -- & 0.99 & 3 \\
\hline 256 & 1.9 & 14 & 13.5 & 2 & - & - & 14.45 & 1 & 25.9 & 4 & 0.97 & 3 \\
\hline 259 & 3.4 & 14 & 10.5 & 4 & 58 & 4 & 16.1 & 4 & 26.5 & 4 & 1.045 & 4 \\
\hline 263 & 3.8 & 10 & 11.4 & 4 & - & -- & 15.75 & 4 & 26.5 & 4 & 1.05 & 4 \\
\hline 265 & 3.4 & 10 & - & - & 58 & 4 & 15.6 & 4 & 26 & 4 & -. & + \\
\hline 266 & 2.8 & 11 & 11.1 & 4 & .- & -. & 17 & 1 & 26.5 & 4 & 1 & 3 \\
\hline 269 & 2.3 & 7 & 11 & 4 & -. & -- & 14 & 0 & 26 & 4 & 0.96 & 2 \\
\hline 277 & 3.8 & 4 & - & - & - & -- & $=$ & - & 26.8 & 4 & 0.98 & 3 \\
\hline 279 & 1.5 & 4 & $=$ & - & - & - & 16.88 & $\overline{2}$ & -- & - & - & $\overline{-}$ \\
\hline 305 & 2.2 & 9 & - & - & -- & - & 15.5 & 4 & 27.67 & 3 & 0.958 & 2 \\
\hline 315 & 3.5 & 6 & - & - & .. & - & 14.8 & 2 & 26.2 & 4 & -. & - \\
\hline 319 & 3.0 & 2 & - & - & 61.6 & 3 & - & - & 27.3 & 3 & $\ldots$ & - \\
\hline 321 & 0.9 & 11 & 7 & 0 & -- & -- & 15.4 & 4 & 24.3 & 1 & - & - \\
\hline 328 & 2.1 & 16 & 8 & 1 & 61 & 3 & 16 & 4 & 24 & 1 & 0.98 & 3 \\
\hline 330 & 2.1 & 10 & 20 & 0 & -- & -. & 16.6 & 2 & 25 & 2 & 1.14 & 2 \\
\hline 333 & 2.5 & 8 & 9.3 & 3 & -- & -. & 16.3 & 3 & -. & - & - & - \\
\hline 336 & 0.0 & 8 & - & - & -- & -- & 9.27 & 0 & 9.94 & 0 & 0.13 & 0 \\
\hline 341 & 2.9 & 14 & 16 & 0 & 64.6 & 2 & 15.3 & 3 & 26.2 & 4 & - & - \\
\hline 356 & 3.8 & 6 & 11.3 & 4 & -- & - & .. & - & 27 & 4 & 0.985 & 3 \\
\hline 366 & 3.3 & 11 & 9.64 & 3 & -- & - & 15.7 & 4 & 27.7 & 3 & - & - \\
\hline 370 & 2.2 & 13 & 10 & 3 & -- & -. & 17.1 & 1 & 26 & 4 & 1.02 & 4 \\
\hline 372 & 2.1 & 15 & 20.4 & 0 & 57 & 4 & 15.8 & 4 & 25.7 & 3 & 0.76 & 0 \\
\hline 374 & 0.0 & 1 & - & $=$ & -- & - & - & - & 30 & 0 & -. & - \\
\hline 375 & 4.0 & 4 & - & - & - & - & 15.44 & 4 & $\cdots$ & - & $\overline{-}$ & - \\
\hline
\end{tabular}


Table 6. Laboratory performance ratings for standard reference sample M-162 (major constituents) -- continued [MPV, most probable value; Lab, laboratory identification number; OLR, overall laboratory rating for all rated analyses; $\mathrm{mg} / \mathrm{L}$, milligrams per liter; $\mu \mathrm{g} / \mathrm{L}$, micrograms per liter; $\mu \mathrm{S} / \mathrm{cm}$, microsiemens per centimeter at 25 degrees Celsius; $V / 16$, number of rated analyses out of 16 possible; RV, reported value; <, less than; NR, not rated; --, not reported.]

\begin{tabular}{|llll|}
\hline Rating & Absolute Z-value & Rating & Absolute Z-value \\
4 (Excellent) & $0.00-0.50$ & 1 (Marginal) & $1.51-2.00$ \\
3 (Good) & $0.51-1.00$ & 0 (Unsatisfactory) & greater than 2.00 \\
2 (Satisfactory) & $1.01-1.50$ & NR (Not Rated) & \\
\hline
\end{tabular}

\begin{tabular}{|c|c|c|c|c|c|c|c|c|c|c|c|}
\hline \multirow[b]{2}{*}{ Lab } & $\begin{array}{r}\text { Analyte }= \\
M P V= \\
\text { F-pseudosigma }=\end{array}$ & \multicolumn{2}{|c|}{$\begin{array}{l}\text { Potassium } \\
3.20 \mathrm{mg} / \mathrm{L} \\
0.17 \\
\end{array}$} & \multicolumn{2}{|c|}{$\begin{array}{l}\text { Magnesium } \\
5.80 \mathrm{mg} / \mathrm{L} \\
0.23\end{array}$} & \multicolumn{2}{|c|}{$\begin{array}{l}\text { Sodlum } \\
24.0 \mathrm{mg} / \mathrm{L} \\
0.8 \\
\end{array}$} & \multicolumn{2}{|c|}{$\begin{array}{l}\text { pH } \\
6.60 \\
0.21\end{array}$} & \multicolumn{2}{|c|}{$\begin{array}{c}\text { Residue on } \\
\text { Evaporation } \\
188 \mathrm{mg} / \mathrm{L} \\
17 \\
\end{array}$} \\
\hline & & RV & Rsting & RV & Rating & AV & Rating & $\mathrm{RV}$ & Rating & RV & Rating \\
\hline 1 & & 3.11 & 3 & 5.82 & 4 & 23.93 & 4 & 6.76 & 4 & 184 & 4 \\
\hline 4 & & - & - & - & -. & - & - & -- & -- & - & $\omega$ \\
\hline 5 & & $<1.00$ & 0 & 1.1 & 0 & 1.76 & 0 & 6.68 & 4 & 196 & 4 \\
\hline 8 & & 3.4 & 2 & 6.2 & 2 & 24.1 & 4 & 7.27 & 0 & 189.2 & 4 \\
\hline 10 & & 3.3 & 3 & 5.8 & 4 & 24 & 4 & 6.5 & 4 & 174 & 3 \\
\hline 12 & & $\overline{1}$ & - & 6.8 & 0 & 26 & 1 & 6.6 & 4 & 58 & 0 \\
\hline 16 & & 3.2 & 4 & 6 & 3 & 23 & 3 & 6.43 & 4 & 119 & 0 \\
\hline 23 & & 3.12 & 4 & 5.76 & 4 & 23.9 & 4 & 6.8 & 3 & - & - \\
\hline 24 & & 3.32 & 3 & 5.85 & 4 & 24.3 & 4 & 6.4 & 3 & - & - \\
\hline 25 & & 0.99 & 0 & 1.09 & 0 & 1.65 & 0 & 6.79 & 3 & 272 & 0 \\
\hline 26 & & 3.12 & 4 & 5.93 & 4 & 24.3 & 4 & -- & - & 178 & 3 \\
\hline 32 & & 2.98 & 2 & 5.6 & 3 & 22.1 & 1 & 6.52 & 4 & 214 & 1 \\
\hline 33 & & 3.29: & 3 & 6.35 & 1 & 23.4 & 4 & 6.99 & 2 & - & - \\
\hline 38 & & 3.31 & 3 & 5.74 & 4 & 23.7 & 4 & 6.8 & 3 & - & - \\
\hline 42 & & 3.01 & 2 & 5.46 & 2 & 21.8 & 1 & 6.55 & 4 & - & - \\
\hline 45 & & 3.25 & 4 & 5.82 & 4 & 23.9 & 4 & 6.48 & 4 & 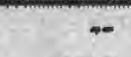 & - \\
\hline 46 & & 3.05 & 3 & 5.6 & 3 & 24.1 & 4 & 6.67 & 4 & 186 & 4 \\
\hline 59 & & 3.3 & 3 & 5.5 & 2 & 23.9 & 4 & 6.53 & 4 & 197 & 3 \\
\hline 64 & & 3.28 & 4 & 5.88 & 4 & 24.1 & 4 & 6.9 & 3 & - & - \\
\hline 70 & & 3.18 & 4 & 5.78 & 4 & 24.6 & 4 & 6.55 & 4 & 190 & 4 \\
\hline 76 & & 3.26 & 4 & - & -- & 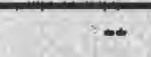 & $\overline{-}$ & -- & - & 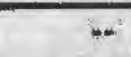 & $=$ \\
\hline 85 & & 3.47 & 1 & 6.04 & 3 & 24.5 & 4 & 6.54 & 4 & 163 & 2 \\
\hline 86 & & 3.41 & 2 & 6.05 & 3 & 25.3 & 2 & 6.59 & 4 & - & - \\
\hline 89 & & 3.37 & 3 & 5.93 & 4 & 24.2 & 4 & 6.5 & 4 & 166 & 2 \\
\hline 97 & & 3.2 & 4 & 5.94 & 4 & 23.2 & 3 & 6.7 & 4 & - & - \\
\hline 102 & & 1.54 & 0 & 5.54 & 3 & 76 & 0 & - & - & $\cdots$ & - \\
\hline 105 & & 3.45 & 2 & 6.08 & 3 & 23.8 & 4 & 6.4 & 3 & 220 & 1 \\
\hline 109 & & 3.2 & 4 & 5.85 & 4 & 21.75 & 1 & 6.48 & 4 & 189 & 4 \\
\hline 113 & & 3.16 & 4 & 5.73 & 4 & 23.2 & 3 & - & -- & 185.7 & 4 \\
\hline 121 & & - & $=$ & 5.6 & 3 & 23 & 3 & -- & - & - & $=$ \\
\hline 134 & & 3.25 & 4 & 5.807 & 4 & 23.87 & 4 & 6.623 & 4 & 194 & 4 \\
\hline 138 & & 3.12 & 4 & 5.86 & 4 & 23.8 & 4 & 6.7 & 4 & 178 & 3 \\
\hline 142 & & 3.16 & 4 & 5.71 & 4 & 23.9 & 4 & 5.9 & 0 & 173 & 3 \\
\hline 146 & & 3.83 & 0 & 5.9 & 4 & 24.9 & 3 & 6.47 & 4 & 208 & 2 \\
\hline 149 & & - & - & .- & -. & $\ldots$ & - & 6.7 & 4 & - & - \\
\hline 180 & & 3.14 & 4 & 5.82 & 4 & 24 & 4 & 6.78 & 3 & - & - \\
\hline 183 & & - & - & -- & -- & - & - & 6.36 & 3 & - & -. \\
\hline 190 & & 3.28 & 4 & 5.92 & 4 & 24.6 & 4 & 6.21 & 2 & 197 & 3 \\
\hline 193 & & 3.32 & 3 & 5.85 & 4 & 24 & 4 & 6.75 & 4 & - & - \\
\hline 208 & & - & -- & -- & - & - & - & - & -- & - & $\cdots$ \\
\hline 212 & & 3.6 & 0 & 5.5 & 2 & 23.5 & 4 & 6.7 & 4 & 180 & 4 \\
\hline 220 & & - & $\ldots$ & 5.99 & 3 & 24.101 & 4 & - & -- & -.. & - \\
\hline 224 & & 2.986 & 2 & 6.106 & 2 & 25.046 & 3 & 6.64 & 4 & 217.5 & 1 \\
\hline 227 & & $=$ & $=$ & -- & - & - & - & 6.78 & 3 & 188 & 4 \\
\hline 234 & & 3.15 & 4 & 5.97 & 3 & 24.4 & 4 & 6.4 & 3 & 123 & 0 \\
\hline
\end{tabular}


Table 6. Laboratory performance ratings for standard reference sample M-162 (major constituents) -- continued [MPV, most probable value; Lab, laboratory identification number; OLR, overall iaboratory rating for all rated anaiyses; $\mathrm{mg} / \mathrm{L}$, milligrams per liter; $\mu \mathrm{g} / \mathrm{L}$, micrograms per liter; $\mu \mathrm{S} / \mathrm{cm}$, microsiemens per centimeter at 25 degrees Celsius; $\mathrm{V} / 16$, number of rated analyses out of 16 possible; RV, reported value; <, less than; NR, not rated; --, not reported.]

\begin{tabular}{|lcll|}
\hline Rating & Absolute Z-value & Rating & Absolute Z-value \\
4 (Excellent) & $0.00-0.50$ & 1 (Marginal) & $1.51-2.00$ \\
3 (Good) & $0.51-1.00$ & 0 (Unsatisfactory) & greater than 2.00 \\
2 (Satisfactory) & $1.01-1.50$ & NR (Not Rated) & \\
\hline
\end{tabular}

\begin{tabular}{|c|c|c|c|c|c|c|c|c|c|c|c|}
\hline \multirow[b]{2}{*}{ Lab } & \multirow{2}{*}{$\begin{array}{r}\text { Analyte }= \\
\text { MPV }= \\
\text { F-pseudosigma }= \\
\end{array}$} & \multicolumn{2}{|c|}{$\begin{array}{l}\text { Potassium } \\
3.20 \mathrm{mg} / \mathrm{L} \\
0.17\end{array}$} & \multicolumn{2}{|c|}{$\begin{array}{l}\text { Magneslum } \\
5.80 \mathrm{mg} / \mathrm{L} \\
0.23\end{array}$} & \multicolumn{2}{|c|}{$\begin{array}{l}\text { Sodlum } \\
24.0 \mathrm{mg} / \mathrm{L} \\
0.8\end{array}$} & \multicolumn{2}{|c|}{$\begin{array}{l}\text { pH } \\
6.60 \\
0.21\end{array}$} & \multicolumn{2}{|c|}{$\begin{array}{l}\text { Residue on } \\
\text { Evaporation } \\
188 \mathrm{mg} / \mathrm{L} \\
17\end{array}$} \\
\hline & & $\mathrm{AV}$ & Rating & RV & Rating & RV & Rating & RV & Rating & RV & Rating \\
\hline 245 & & 3.071 & 3 & 5.672 & 4 & - & - & - & -- & - & - \\
\hline 247 & & 2.98 & 2 & 5.31 & 1 & 21.8 & 1 & 6.84 & 3 & 174 & 3 \\
\hline 255 & & - & - & 5.74 & 4 & - & - & - & -- & - & - \\
\hline 256 & & 3.02 & 2 & - & - & 24.5 & 4 & 5.75 & 0 & 198 & 3 \\
\hline 259 & & 3.02 & 2 & 3 & 0 & 24.5 & 4 & 6.81 & 3 & - & -. \\
\hline 263 & & - & - & 5.7 & 4 & - & $\overline{-}$ & 6.63 & 4 & 175 & 3 \\
\hline 265 & & 3.15 & 4 & 5.7 & 4 & 24 & 4 & - & - & - & - \\
\hline 266 & & 3.5 & 1 & 5.3 & 1 & 24.6 & 4 & -- & - & 188 & 4 \\
\hline 269 & & - & - & 7.29 & 0 & - & - & 6.25 & 2 & - & - \\
\hline 277 & & - & $=$ & - & - & - & - & - & - & 189 & 4 \\
\hline 279 & & 3.76 & 0 & 6.38 & 1 & 24,84 & 3 & - & 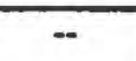 & - & 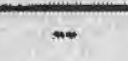 \\
\hline 305 & & 3.96 & 0 & 5.78 & 4 & 21.2 & 0 & 6.5 & 4 & - & - \\
\hline 315 & & 3.3 & 3 & 5.8 & 4 & 23.9 & 4 & -- & - & - & - \\
\hline 319 & & - & - & - & -- & - & - & - & - & - & - \\
\hline 321 & & 3.4 & 2 & 5.1 & 0 & 20.1 & 0 & 5.76 & 0 & - & $=$ \\
\hline 328 & & 1.5 & 0 & 6 & 3 & 25 & 3 & 6.05 & 1 & 140 & 0 \\
\hline 330 & & 3.41 & 2 & 5.9 & 4 & 25.2 & 3 & 6.99 & 2 & 186 & 4 \\
\hline 333 & & 3.93 & 0. & 6.02 & 3 & - & - & 6.58 & 4 & - & - \\
\hline 336 & & 0.79 & 0. & 0.99 & 0 & 2.25 & 0 & 5.68 & 0 & - & - \\
\hline 341 & & 3.2 & 4 & 5.7 & 4 & 25 & 3 & 6.54 & 4 & 208 & 2 \\
\hline 356 & & - & - & -- & - & - & - & - & -- & 192.6 & 4 \\
\hline 366 & & 3.26 & 4 & 5.76 & 4 & 23.5 & 4 & 6.67 & 4 & 160 & 1 \\
\hline 370 & & 3.7 & 0 & 5.95 & 4 & 26.6 & 0 & 7.12 & 1 & 220 & 1 \\
\hline 372 & & 2.76 & 0 & 5.59 & 3 & - & - & 6.73 & 4 & 207 & 2 \\
\hline 374 & & - & - & -- & - & - & 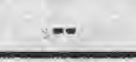 & -- & - & -. & - \\
\hline 375 & & 3.193 & 4 & 5.8 & 4 & 23.975 & 4 & - & $\cdots$ & 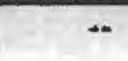 & 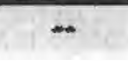 \\
\hline
\end{tabular}


Table 6. Laboratory performance ratings for standard reference sample M-162 (major constituents) -- continued [MPV, most probable value; Lab, laboratory identification number; OLR, overall laboratory rating for all rated analyses; $\mathrm{mg} / \mathrm{L}$, milligrams per liter; $\mu \mathrm{g} / \mathrm{L}$, micrograms per liter; $\mu \mathrm{S} / \mathrm{cm}$, microsiemens per centimeter at 25 degrees Celsius; V/16, number of rated analyses out of 16 possible; RV, reported value; <, less than; NR, not rated; --, not reported.]

\begin{tabular}{|lcll|}
\hline Rating & Absolute Z-value & Rating & Absolute Z-value \\
4 (Excellent) & $0.00-0.50$ & 1 (Marginal) & $1.51-2.00$ \\
3 (Good) & $0.51-1.00$ & 0 (Unsatisfactory) & greater than 2.00 \\
2 (Satisfactory) & $1.01-1.50$ & NR (Not Rated) & \\
\hline
\end{tabular}

\begin{tabular}{|c|c|c|c|c|c|c|c|c|c|c|c|}
\hline \multirow[b]{2}{*}{ Lab } & $\begin{array}{r}\text { Analyte }= \\
M P V= \\
\text { F-pseudosigma }=\end{array}$ & \multicolumn{2}{|c|}{$\begin{array}{l}\text { Sillca } \\
13.9 \mathrm{mg} / \mathrm{L} \\
0.6\end{array}$} & \multicolumn{2}{|c|}{$\begin{array}{l}\text { Sulfate } \\
13.4 \mathrm{mg} / \mathrm{L} \\
0.9\end{array}$} & \multicolumn{2}{|c|}{$\begin{array}{c}\text { Speciflc } \\
\text { Conductance } \\
284 \mu \mathrm{S} / \mathrm{cm} \\
6\end{array}$} & \multicolumn{2}{|c|}{$\begin{array}{l}\text { Strontium } \\
93.8 \mu \mathrm{g} / \mathrm{L} \\
3.2\end{array}$} & \multicolumn{2}{|c|}{$\begin{array}{c}\text { Phosphorus as P } \\
0.510 \mathrm{mg} / \mathrm{L} \\
0.024\end{array}$} \\
\hline & & RV & Rating & RV & Rating & RV & Rating & RV & Rating & RV & Rating \\
\hline 1 & & 13.9 & 4 & 13.28 & 4 & 286 & 4 & 93.88 & 4 & 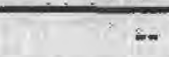 & - \\
\hline 4 & & - & - & 11.3 & 0 & - & - & .. & -- & - & - \\
\hline 5 & & 0.547 & 0 & 13.05 & 4 & 254 & 0 & 23.7 & 0 & 0.51 & 4 \\
\hline 8 & & 14.2 & 4 & 12.6 & 3 & 168.3 & 0 & 100 & 2 & 0.72 & 0 \\
\hline 10 & & 13.8 & 4 & 13.7 & 4 & 282 & 4 & - & -- & - & - \\
\hline 12 & & - & - & 4 & 0 & 308 & 1 & - & -- & 0.49 & 3 \\
\hline 16 & & - & -. & 14.3 & 2 & 295 & 3 & 90 & 3 & 0.628 & 0 \\
\hline 23 & & - & -. & 1.3 & 0 & 291 & 4 & -- & .- & 0.48 & 2 \\
\hline 24 & & 14.9 & 2 & 14.2 & 3 & 278 & 4 & 96.8 & 3 & - & - \\
\hline 25 & & 10.8 & 0 & 12.1 & 2 & 286 & 4 & 90 & 3 & - & - \\
\hline 26 & & - & $\pi$ & 12.5 & 2 & 278 & $\overline{4}$ & - & - & - & 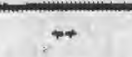 \\
\hline 32 & & 13.8 & 4 & 13.8 & 4 & 282 & 4 & 95 & 4 & 0.61 & 0 \\
\hline 33 & & 6.12 & 0 & 14.5 & 2 & 316.7 & 0 & 91.2 & 3 & - & - \\
\hline 38 & & 13.8 & 4 & -- & -- & 280.7 & 4 & -- & -- & 0.524 & 3 \\
\hline 42 & & 12.9 & 2 & 11.3 & 0 & 302 & 2 & 113 & 0 & 0.505 & 4 \\
\hline 45 & & - & -. & 12.9 & 3 & 290 & $\overline{4}$ & -- & -- &.- & $=$ \\
\hline 46 & & - & - & 13.3 & 4 & 282 & 4 & -. & .- & 0.522 & 4 \\
\hline 59 & & .. & - & 13.8 & 4 & 303 & 2 & 93.7 & 4 & - & - \\
\hline 64 & & 14.4 & 3 & 13.7 & 4 & 227 & 0 & -- & -- & 0.526 & 3 \\
\hline 70 & & 14.3 & 3 & 13.6 & 4 & 275 & 3 & -- & -- & 0.49 & 3 \\
\hline 76 & & - & 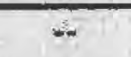 & - & -. & 280 & 4 & 92.06 & 4 & - & - \\
\hline 85 & & 15.5 & 0 & 14 & 3 & 274 & 3 & 94 & 4 & 0.493 & 3 \\
\hline 86 & & - & -. & 13.5 & 4 & 293 & 3 & 94 & 4 & 0.506 & 4 \\
\hline 89 & & 15 & 1 & 13.7 & 4 & 280 & 4 & -- & -- & 0.509 & 4 \\
\hline 97 & & 15.6 & 0 & 9.07 & 0 & 284 & 4 & 92.2 & 4 & - & - \\
\hline 102 & & 13.6 & 4 & 13.7 & 4 & 298 & 3 & 82 & 0 & 0.519 & $\overline{4}$ \\
\hline 105 & & 14,488 & 3 & 12.6 & 3 & 277 & 4 & 94 & 4 & 0.464 & 1 \\
\hline 109 & & - & - & 14.19 & 3 & 283 & 4 & -- & -- & -. & - \\
\hline 113 & & 13.7 & 4 & 13.3 & 4 & 285 & 4 & 91.4 & 3 & 0.507 & 4 \\
\hline 121 & & 13.6 & 4 & -- & -- & - & - & 94 & 4 & - & - \\
\hline 134 & & 13.98 & 4 & 13.11 & $\overline{4}$ & 283 & 4 & 93.08 & 4 & 0.528 & $\overline{3}$ \\
\hline 138 & & 14.6 & 3 & 13.3 & 4 & 284 & 4 & 93.8 & 4 & 0.525 & 3 \\
\hline 142 & & 13.8 & 4 & 13.5 & 4 & 286 & 4 & 102 & 1 & 0.523 & 4 \\
\hline 146 & & - & - & 13.5 & 4 & 258 & 1 & -- & -- & -. & -. \\
\hline 149 & & $\ldots$ & - & 12.3 & 2 & 280 & 4 & -- & -- & 0.521 & 4 \\
\hline 180 & & - & $\overline{-}$ & 13.4 & 4 & 194 & $\overline{0}$ & -. & -- & 0.496 & 3 \\
\hline 183 & & - & - & 14.5 & 2 & 290 & 4 & .- & -- & 0.528 & 3 \\
\hline 190 & & 14.1 & 4 & 13.5 & 4 & 282 & 4 & $<0.01$ & 0 & 0.505 & 4 \\
\hline 193 & & - & - & -- & -- & 285 & 4 & - & -. & - & $\ldots$ \\
\hline 208 & & - & - & 12.6 & 3 & - & - & -. & -- & -- & .- \\
\hline 212 & & 10.2 & $\overline{0}$ & 12.7 & 3 & 256 & $T$ & - & - & 0.45 & 0 \\
\hline 220 & & 14.21 & 4 & 13.072 & 4 & .. & $\ldots$ & -- & -- & 0.513 & 4 \\
\hline 224 & & 13.73 & 4 & 13.41 & 4 & 280 & 4 & - & -- & 0.484 & 2 \\
\hline 227 & & - & - & -- & -- & 282 & 4 & -- & -- & 0.503 & 4 \\
\hline 234 & & 13.9 & 4 & 13.4 & 4 & 278 & 4 & 94.5 & 4 & 0.55 & 1 \\
\hline
\end{tabular}


Table 6. Laboratory performance ratings for standard reference sample M-162 (major constituents) -- continued [MPV, most probable value; Lab, laboratory identification number; OLR, overall laboratory rating for all rated analyses; $\mathrm{mg} / \mathrm{L}$, milligrams per liter; $\mu \mathrm{g} / \mathrm{L}$, micrograms per liter; $\mu \mathrm{S} / \mathrm{cm}$, microsiemens per centimeter at 25 degrees Celsius; $\mathrm{V} / 16$, number of rated analyses out of 16 possible; RV, reported value; <, less than; NR, not rated; --, not reported.]

\begin{tabular}{|llll|}
\hline Rating & Absolute Z-value & Rating & Absolute Z-value \\
\hline (Excellent) & $0.00-0.50$ & 1 (Marginal) & $1.51-2.00$ \\
3 (Good) & $0.51-1.00$ & 0 (Unsatisfactory) & greater than 2.00 \\
2 (Satisfactory) & $1.01-1.50$ & NR (Not Rated) & \\
\hline
\end{tabular}

\begin{tabular}{|c|c|c|c|c|c|c|c|c|c|c|c|}
\hline \multirow[b]{2}{*}{ Lab } & \multirow{2}{*}{$\begin{array}{r}\text { Analyte }= \\
M P V= \\
\text { F-pseudosigma }=\end{array}$} & \multicolumn{2}{|c|}{$\begin{array}{l}\text { Sillca } \\
13.9 \mathrm{mg} / \mathrm{L} \\
0.6\end{array}$} & \multicolumn{2}{|c|}{$\begin{array}{l}\text { Sulfate } \\
13.4 \mathrm{mg} / \mathrm{L} \\
0.9\end{array}$} & \multicolumn{2}{|c|}{$\begin{array}{c}\text { Specific } \\
\text { Conductance } \\
284 \mu \mathrm{S} / \mathrm{cm} \\
6\end{array}$} & \multicolumn{2}{|c|}{$\begin{array}{l}\text { Strontlum } \\
93.8 \mu \mathrm{g} / \mathrm{L} \\
3.2\end{array}$} & \multicolumn{2}{|c|}{$\begin{array}{l}\text { Phosphorus as P } \\
0.510 \mathrm{mg} / \mathrm{L} \\
0.024\end{array}$} \\
\hline & & $\mathrm{RV}$ & Rating & RV & Rating & RV & Rating & RV & Rating & RV & Rating \\
\hline 245 & & - & - & -- & - & - &.- & 89.5 & 3 & - & - \\
\hline 247 & & 15.1 & 1 & 12.7 & 3 & 288 & 4 & 80 & 0 & 0.51 & 4 \\
\hline 255 & & - & - & 14 & 3 & - & - & - & - & - & - \\
\hline 256 & & 11.88 & 0 & 16.43 & 0 & 285 & 4 & 88.7 & 2 & 0.57 & 0 \\
\hline 259 & & 13,8 & 4 & 13.4 & 4 & 284 & 4 & 98 & 3 & 0.506 & 4 \\
\hline 263 & & 13.6 & 4 & 13.9 & 3 & 286 & 4 & - & - & - & - \\
\hline 265 & & 13.9 & 4 & 14.5 & 2 & - & - & 94 & 4 & - & - \\
\hline 266 & & 14 & 4 & 15 & 1 & 290 & 4 & -- & -- & - & - \\
\hline 269 & & - & - & -- & - & 284 & 4 & $\cdots$ & - & $\infty$ & - \\
\hline 277 & & - & - & 13.2 & 4 & ... & 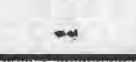 & - & $\cdots$ & - & - \\
\hline 279 & & 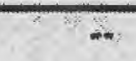 & $*$ & $=$ & - & $=$ & $\because$ & - & - & 4 & 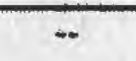 \\
\hline 305 & & $\cdots$ & - & 12.57 & 3 & - & - & -- & - & - & - \\
\hline 315 & & - & -. & 13.7 & 4 & - & - & - & - & - & - \\
\hline 319 & & - & - & - & - & - & - & - & - & - & - \\
\hline 321 & & 12.9 & 2 & 11.64 & 1 & 247 & 0 & - & - & 0.437 & 0 \\
\hline 328 & & 14.9 & 2 & 13 & 4 & 284 & 4 & 86 & $\overline{1}$ & 0.49 & 3 \\
\hline 330 & & - & - & 18.2 & 0 & - & - & -- & -- & - & - \\
\hline 333 & & 15 & 1 & -- & - & 266 & 2 & 94.2 & 4 & ** & - \\
\hline 336 & & - & $\ldots$ & 0.999 & 0 & - & - & -- & - & - & - \\
\hline 341 & & - & - & 10.5 & 0 & 285 & 4 & 94.6 & 4 & 0.533 & 3 \\
\hline 356 & & - & $=$ & 13.1 & 4 & 284 & 4 & - & -- & - & 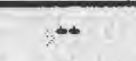 \\
\hline 366 & & - & - & 13.9 & 3 & 271 & 3 & - & - & 0.493 & 3 \\
\hline 370 & & 6.9 & 0 & 14.6 & 2 & 288 & 4 & -. & -- & 0.52 & 4 \\
\hline 372 & & 14.3 & 3 & 11.4 & 0 & 286 & 4 & 87 & 2 & 0.59 & 0 \\
\hline 374 & & - & - & -- & - & - & - & -. & - & - & $\cdots$ \\
\hline 375 & & - & - & - & -- & - & - & -- & -- & - & 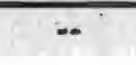 \\
\hline
\end{tabular}


Table 6. Laboratory performance ratings for standard reference sample M-162 (major constituents) -- continued IMPV, most probable value; Lab, laboratory identification number; OLR, overall laboratory rating for all rated analyses; $\mathrm{mg} / \mathrm{L}$, milligrams per liter; $\mu \mathrm{g} / \mathrm{L}$, micrograms per liter; $\mu \mathrm{S} / \mathrm{cm}$, microsiemens per centimeter at 25 degrees Celsius; V/16, number of rated analyses out of 16 possible; RV, reported value; <, less than; NR, not rated; --, not reported.]

\begin{tabular}{|llll|}
\hline Rating & Absolute Z-value & Rating & Absolute Z-value \\
4 (Excellent) & $0.00-0.50$ & 1 (Marginal) & $1.51-2.00$ \\
3 (Good) & $0.51-1.00$ & 0 (Unsatisfactory) & greater than 2.00 \\
2 (Satisfactory) & $1.01-1.50$ & NR (Not Rated) & \\
\hline
\end{tabular}

\begin{tabular}{|c|c|c|c|}
\hline & $\begin{array}{r}\text { Analyte }= \\
\text { MPV }= \\
\text { F-pseudosigma }=\end{array}$ & $\begin{array}{l}\text { Vana } \\
9.74 \\
1.04\end{array}$ & ligm \\
\hline Lab & & $\mathrm{RV}$ & Rating \\
\hline 1 & & 10.36 & 3 \\
\hline 4 & & - & - \\
\hline 5 & & $<4.00$ & 0 \\
\hline 8 & & 9.9 & 4 \\
\hline 10 & & - & - \\
\hline 12 & & - & - \\
\hline 16 & & 11 & 2 \\
\hline 23 & & - & - \\
\hline 24 & & - & - \\
\hline 25 & & $<19$ & NR \\
\hline 26 & & - & - \\
\hline 32 & & 9.9 & 4 \\
\hline 33 & & - & - \\
\hline 38 & & - & $\ldots$ \\
\hline 42 & & 9.6 & 4 \\
\hline 45 & & 9.47 & 4 \\
\hline 46 & & - & - \\
\hline 59 & & 8.97 & 3 \\
\hline 64 & & - & 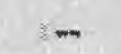 \\
\hline 70 & & - & - \\
\hline 76 & & 9.88 & 4 \\
\hline 85 & & 15 & 0 \\
\hline 86 & & 8.5 & 2 \\
\hline 89 & & 12 & 0 \\
\hline 97 & & $<78$ & NR \\
\hline 102 & & 14 & 0 \\
\hline 105 & & $<20.0$ & NR \\
\hline 109 & & - & - \\
\hline 113 & & - & $\omega$ \\
\hline 121 & & 11 & 2 \\
\hline 134 & & 9.19 & 3 \\
\hline 138 & & 8.75 & 3 \\
\hline 142 & & 9.48 & 4 \\
\hline 146 & & 9.02 & 3 \\
\hline 149 & & - & - \\
\hline 180 & & 9.58 & 4 \\
\hline 183 & & - & - \\
\hline 190 & & - & - \\
\hline 193 & & - & - \\
\hline 208 & & - & - \\
\hline 212 & & - & - \\
\hline 220 & & - & - \\
\hline 224 & & $m$ & - \\
\hline 227 & & - & $\cdots$ \\
\hline 234 & & 10.4 & 3 \\
\hline
\end{tabular}

\begin{tabular}{|c|c|c|c|}
\hline & $\begin{array}{r}\text { Analyte }= \\
\text { MPV }= \\
\text { F-pseudosigma }=\end{array}$ & $\begin{array}{l}\text { Vana } \\
9.74 \\
1.04\end{array}$ & Ilum \\
\hline Lab & & RV & Rating \\
\hline 245 & & 10.1 & 4 \\
\hline 247 & & - & $\because$ \\
\hline 255 & & $\ldots$ & - \\
\hline 256 & & 8.65 & 2 \\
\hline 259 & & - & - \\
\hline 263 & & - & $\overline{-}$ \\
\hline 265 & & 14 & 0 \\
\hline 266 & & - & - \\
\hline 269 & & - & - \\
\hline 277 & & - & - \\
\hline 279 & & - & - \\
\hline 305 & & 3.2 & 0 \\
\hline 315 & & - & - \\
\hline 319 & & -. & - \\
\hline 321 & & - & - \\
\hline 328 & & 8 & 1 \\
\hline 330 & & - & - \\
\hline 333 & & $\omega$ & - \\
\hline 336 & & n & - \\
\hline 341 & & 9.9 & 4 \\
\hline 356 & & + & - \\
\hline 366 & & - & - \\
\hline 370 & & - & - \\
\hline 372 & & 9 & 3 \\
\hline 374 & & $-\infty$ & - \\
\hline 375 & & - & - \\
\hline
\end{tabular}


Table 7. Laboratory performance ratings for standard reference sample $\mathbf{N}-73$ (nutrient constituents)

[MPV, most probable value; Lab, laboratory identification number; OLR, overall laboratory rating for all rated analyses; $\mathrm{mg} / \mathrm{L}$, milligrams per liter; V/5, number of rated analyses out of 5 possible; RV, reported value; <, less than; NR, not rated; --, not reported.]

\begin{tabular}{|llll|}
\hline Rating & Absolute Z-value & Rating & Absolute Z-value \\
4 (Excellent) & $0.00-0.50$ & 1 (Marginal) & $1.51-2.00$ \\
3 (Good) & $0.51-1.00$ & 0 (Unsatisfactory) & greater than 2.00 \\
2 (Satisfactory) & $1.01-1.50$ & NR (Not Rated) & \\
\hline
\end{tabular}

\begin{tabular}{|c|c|c|c|c|c|c|c|c|c|c|c|c|}
\hline \multirow[b]{2}{*}{ Lab } & \multicolumn{2}{|c|}{$\begin{array}{r}\text { Analyte }= \\
\text { MPV }= \\
\text { F-pseudosigma }=\end{array}$} & \multicolumn{2}{|c|}{$\begin{array}{l}\text { Ammonia as } \mathrm{N} \\
0.127 \mathrm{mg} / \mathrm{L} \\
0.010\end{array}$} & \multicolumn{2}{|c|}{$\begin{array}{l}\text { Ammonia + } \\
\text { Organic N as N } \\
0.140 \mathrm{mg} / \mathrm{L} \\
0.022\end{array}$} & \multicolumn{2}{|c|}{$\begin{array}{l}\text { Nituate as } \mathrm{N} \\
0.126 \mathrm{mg} / \mathrm{l} \\
0.007\end{array}$} & \multicolumn{2}{|c|}{$\begin{array}{c}\text { Phosphorus as P } \\
0.132 \mathrm{mg} / \mathrm{L} \\
0.009\end{array}$} & \multicolumn{2}{|c|}{$\begin{array}{c}\text { Orthophosphate } \\
\text { as P } \\
0.128 \mathrm{mg} / \\
0.004\end{array}$} \\
\hline & OLR & $\mathrm{V} / 5$ & AV & Rating & RV & Rating & RV & Rating & $\mathrm{RV}$ & Rating & $\mathrm{PV}$ & Rating \\
\hline 1 & 3.4 & 5 & 0.119 & 3 & 0.119 & 3 & 0.122 & 3 & 0.129 & 4 & 0.128 & 4 \\
\hline 5 & 2.0 & 5 & 0.154 & 0 & 0.16 & 3 & 0.153 & 0 & 0.136 & 4 & 0.133 & 3 \\
\hline 8 & 1.8 & 5 & 0.17 & 0 & 0.17 & 2 & 0.12 & 3 & 0.15 & 0 & 0.13 & 4 \\
\hline 10 & 3.6 & 5 & 0.13 & 4 & 0.13 & 4 & 0.13 & 3 & 0.134 & 4 & 0.132 & 3 \\
\hline 12 & 2.6 & 5 & 0.12 & 3 & 0.23 & 0 & 0.12 & 3 & 0.126 & 3 & 0.127 & 4 \\
\hline 16 & 3.2 & 5 & 0.12 & 3 & 0.15 & 4 & 0.12 & 3 & 0.134 & 4 & 0.119 & 2 \\
\hline 21 & 3.8 & 5 & 0.118 & 3 & 0.15 & 4 & 0.129 & 4 & 0.132 & 4 & 0.129 & 4 \\
\hline 23 & 0.2 & 5 & 0.098 & 0 & 0.308 & 0 & 0.141 & 0 & 0.105 & 0 & 0.116 & 1 \\
\hline 25 & 3.0 & 5 & 0.13 & 4 & 0.14 & 4 & 0.113 & 1 & 0.12 & 2 & 0.128 & 4 \\
\hline 26 & 0.0 & 2 & $<0.4$ & NR & -- & - & 0.11 & 0 & - & - & 0.11 & 0 \\
\hline 31 & 4.0 & 5 & 0,124 & 4 & 0.146 & 4 & 0.128 & 4 & 0.133 & 4 & 0.129 & 4 \\
\hline 33 & 1.7 & 3 & 0.132 & 4 & - & - & 0.14 & 1 & -- & -- & 0.15 & 0 \\
\hline 38 & 3.6 & 5 & 0.133 & 3 & 0.13 & 4 & 0.121 & 3 & 0.134 & 4 & 0.126 & 4 \\
\hline 42 & 1.3 & 3 & - & - & -- & - & 0.103 & 0 & 0.131 & 4 & 0.142 & 0 \\
\hline 45 & 2.5 & 4 & 0.136 & 3. & -. & - & 0.15 & 0 & 0.14 & 3 & 0.129 & 4 \\
\hline 46 & 2.5 & 4 & 0.116 & 2 & - & - & 0.126 & 4 & 0.135 & 4 & 0.151 & 0 \\
\hline 51 & 3.8 & 5 & 0.13 & 4 & 0.131 & 4 & 0.12 & 3 & 0.13 & 4 & 0.126 & 4 \\
\hline 59 & 3.2 & 5 & 0.12 & 3 & 0.135 & 4 & 0.128 & 4 & 0.131 & 4 & 0.138 & 1 \\
\hline 64 & 2.8 & 4 & 0.14 & 2 & -- & - & 0.12 & 3 & 0.12 & 2 & 0.126 & 4 \\
\hline 70 & 1.4 & 5 & 0.118 & 3 & 0.181 & 1 & 0.144 & 0 & 0.244 & 0 & 0.122 & 3 \\
\hline 72 & 0.8 & 5 & 0.1 & 0 & 0.114 & 2 & 0.113 & 1 & 0.115 & 1 & 0.101 & 0 \\
\hline 85 & 3.6 & 5 & 0.13 & 4 & 0.11 & 2 & 0.125 & 4 & 0.132 & 4 & 0.128 & 4 \\
\hline 86 & 1.3 & 3 & 0.129 & 4 & -- & - & 0.107 & 0 & 0.114 & 0 & - & - \\
\hline 89 & 2.8 & 5 & 0.118 & 3 & 0.17 & 2 & 0.13 & 3 & 0.123 & 2 & 0.13 & 4 \\
\hline 102 & 2.0 & 4 & 0.16 & 0 & $<0.2$ & NR & 0.105 & 0 & 0.132 & 4 & 0.131 & 4 \\
\hline 105 & 1.3 & 4 & 0.12 & 3 & $<1.00$ & NR & 0.15 & 0 & 0.122 & 2 & 0.099 & 0 \\
\hline 110 & 2.0 & 1 & 0.139 & 2 & -- & - & - & - & -- & - & - & - \\
\hline 113 & 3.0 & 5 & 0.099 & 0 & 0.14 & 4 & 0.129 & 4 & 0.139 & 3 & 0.127 & 4 \\
\hline 118 & 3.3 & 3 & 0.118 & 3 & -- & -- & 0.133 & 3 & -- & - & 0.127 & 4 \\
\hline 134 & 3.5 & 4 & 0.126 & 4 & $<0.2$ & NR & 0.127 & 4 & 0.12 & 2 & 0.13 & 4 \\
\hline 138 & 3.8 & 5 & 0.122 & 4 & 0.137 & 4 & 0.123 & 4 & 0.14 & 3 & 0.129 & 4 \\
\hline 142 & 2.8 & 5 & 0.126 & 4 & 0.104 & 1 & 0.132 & 3 & 0.13 & 4 & 0.119 & 2 \\
\hline 146 & 2.5 & 4 & 0.142 & 2 & $<0.200$ & NR & 0.128 & 4 & 0.142 & 2 & 0.137 & 2 \\
\hline 180 & 3.4 & 5 & 0.127 & 4 & 0.139 & 4 & 0.121 & 3 & 0.128 & 4 & 0.136 & 2 \\
\hline 183 & 2.0 & 2 & - & $\therefore$ & $<1$ & NR & $<1$ & NR & 0.143 & 2 & 0.119 & 2 \\
\hline 190 & 3.2 & 5 & 0.118 & 3 & 0.14 & 4 & 0.125 & 4 & 0.121 & 2 & 0.122 & 3 \\
\hline 193 & 3.3 & 3 & 0.116 & 2 & $<0.5$ & NR & 0.126 & 4 & 0.135 & 4 & - & - \\
\hline 198 & 3.0 & 4 & 0.131 & 4 & -- & - & 0.126 & 4 & 0.121 & 2 & 0.12 & 2 \\
\hline 227 & 2.2 & 5 & 0.172 & 0 & 0.16 & 3 & 0.157 & 0 & 0.128 & 4 & 0.128 & 4 \\
\hline 234 & 2.5 & 4 & 0.12 & 3 & -. & - & 0.13 & 3 & 0.155 & 0 & 0.125 & 4 \\
\hline 247 & 3.0 & 5 & 0.13 & 4 & 0.15 & 4 & 0.13 & 3 & 0.15 & 0 & 0.13 & 4 \\
\hline 306 & 0.5 & 4 & 0.14 & 2 & 0.651 & 0 & 0.315 & 0 & 0.167 & 0 & - & - \\
\hline 313 & 3.6 & 5 & 0,119 & 3 & 0.119 & 3 & 0.124 & 4 & 0.13 & 4 & 0.129 & 4 \\
\hline 316 & 1.6 & 5 & 0.1095 & 1 & 0.1607 & 3 & 0.1343 & 2 & 0.1444 & 2 & 0.152 & 0 \\
\hline 318 & 3.8 & 5 & 0.125 & 4 & 0.13 & 4 & 0.13 & 3 & 0.136 & 4 & 0.131 & 4 \\
\hline
\end{tabular}


Table 7. Laboratory performance ratings for standard reference sample $\mathrm{N}-73$ (nutrient constituents) -- continued [MPV, most probable value; Lab, laboratory identification number; OLR, overall laboratory rating for all rated analyses; $\mathrm{mg} / \mathrm{L}$, milligrams per liter; V/5, number of rated analyses out of 5 possible; RV, reported value; <, less than; NR, not rated; --, not reported.]

\begin{tabular}{|lcll|}
\hline Rating & Absolute Z-value & Rating & Absolute Z-value \\
4 (Excellent) & $0.00-0.50$ & 1 (Marginal) & $1.51-2.00$ \\
3 (Good) & $0.51-1.00$ & 0 (Unsatisfactory) & greater than 2.00 \\
2 (Satisfactory) & $1.01-1.50$ & NR (Not Rated) & \\
\hline
\end{tabular}

\begin{tabular}{|c|c|c|c|c|c|c|c|c|c|c|c|c|}
\hline \multirow[b]{2}{*}{ Lab } & \multicolumn{2}{|c|}{$\begin{array}{r}\text { Anaiyte }= \\
\text { MPV }= \\
\text { F-pseudosigma }= \\
\end{array}$} & \multicolumn{2}{|c|}{$\begin{array}{l}\text { Ammonla as } \mathrm{N} \\
0.127 \mathrm{mg} / \mathrm{L} \\
0.010\end{array}$} & \multicolumn{2}{|c|}{$\begin{array}{l}\text { Ammonia + } \\
\text { Organic } \mathrm{N} \text { as } \mathrm{N} \\
0.140 \mathrm{mg} / \mathrm{L} \\
0.022 \\
\end{array}$} & \multicolumn{2}{|c|}{$\begin{array}{l}\text { Nitrate as } \mathrm{N} \\
0.126 \mathrm{mg} / \mathrm{L} \\
0.007\end{array}$} & \multicolumn{2}{|c|}{$\begin{array}{l}\text { Phosphorus as P } \\
0.132 \mathrm{mg} / \mathrm{L} \\
0.009\end{array}$} & \multicolumn{2}{|c|}{$\begin{array}{l}\text { Orthophosphate } \\
\text { as P } \\
0.128 \mathrm{mg} / \mathrm{L} \\
0.004\end{array}$} \\
\hline & OLR & $\mathrm{V} / 5$ & $\mathrm{RV}$ & Rating & RV & Rating & RV & Rating & $\mathrm{RV}$ & Rating & AV & Rating \\
\hline 320 & 3.0 & 5 & 0.13 & 4 & 0.083 & 0 & 0.125 & 4 & 0.133 & 4 & 0.123 & 3 \\
\hline 321 & 2.5 & 4 & 0,13 & 4 & -- & - & 0.11 & 0 & 0.143 & 2 & 0.128 & 4 \\
\hline 328 & 1.6 & 5 & 0.11 & 1 & 0.133 & 4 & 0.12 & 3 & 0.17 & 0 & 0.52 & 0 \\
\hline 333 & 2.0 & 2 & 0.174 & 0 & -. & - & 0.126 & 4 & -- & - & .. & - \\
\hline 341 & 3.4 & 5 & 0.122 & 4 & 0.13 & 4 & 0.123 & 4 & 0.14 & 3 & 0.119 & 2 \\
\hline 366 & 3.0 & 5 & 0.13 & 4 & 0.089 & 0 & 0.127 & 4 & 0.127 & 3 & 0.129 & 4 \\
\hline 370 & 0.8 & 4 & 0.2 & 0 & $<0.5$ & NR & 0.18 & 0 & 0.14 & 3 & 0.143 & 0 \\
\hline 372 & 1.4 & 5 & 0.143 & 1 & 0.255 & 0 & 0.116 & 2 & 0.18 & 0 & 0.13 & 4 \\
\hline 373 & 3.6 & 5 & 0.128 & 4 & 0.143 & 4 & 0.135 & 2 & 0.131 & 4 & 0.126 & 4 \\
\hline 376 & 3.0 & 5 & 0.159 & 0 & 0.157 & 3 & 0.127 & 4 & 0.132 & 4 & 0.126 & 4 \\
\hline 377 & 3.4 & 5 & 0.12 & 3 & 0.12 & 3 & 0.124 & 4 & 0.132 & 4 & 0.132 & 3 \\
\hline
\end{tabular}


Table 8. Laboratory performance ratings for standard reference sample $\mathrm{N}-74$ (nutrient constituents)

[MPV, most probable value; Lab, laboratory number; OLR, overall laboratory rating for all rated analyses; $\mathrm{mg} / \mathrm{L}$, milligrams per liter; V/5, number of rated analyses out of 5 possible; RV, reported value; <, less than; NR, not rated; --, not reported.]

\begin{tabular}{|llll|}
\hline Rating & Absolute Z-value & Rating & Absolute Z-value \\
4 (Excellent) & $0.00-0.50$ & 1 (Marginal) & $1.51-2.00$ \\
3 (Good) & $0.51-1.00$ & 0 (Unsatisfactory) & greater than 2.00 \\
2 (Satisfactory) & $1.01-1.50$ & NR (Not Rated) & \\
\hline
\end{tabular}

\begin{tabular}{|c|c|c|c|c|c|c|c|c|c|c|c|c|}
\hline \multirow[b]{2}{*}{ Lab } & \multicolumn{2}{|c|}{$\begin{array}{r}\text { Analyte }= \\
M P V= \\
\text { F-pseudosigma }=\end{array}$} & \multicolumn{2}{|c|}{$\begin{array}{l}\text { Ammonia as } \mathrm{N} \\
0.760 \mathrm{mg} / \mathrm{L} \\
0.037\end{array}$} & \multicolumn{2}{|c|}{$\begin{array}{l}\text { Ammonla + } \\
\text { Organic } \mathrm{N} \text { as } \mathrm{N} \\
0.838 \mathrm{mg} / \mathrm{L} \\
0.113\end{array}$} & \multicolumn{2}{|c|}{$\begin{array}{l}\text { Nitrate as } \mathrm{N} \\
0.711 \mathrm{mg} / \mathrm{L} \\
0.122\end{array}$} & \multicolumn{2}{|c|}{$\begin{array}{c}\text { Phosphorus as P } \\
0.755 \mathrm{mg} / \mathrm{L} \\
0.031\end{array}$} & \multicolumn{2}{|c|}{$\begin{array}{c}\text { Orthophosphate } \\
\text { as P } \\
0.741 \mathrm{mg} / \mathrm{L} \\
0.035\end{array}$} \\
\hline & OLR & $\mathrm{V} / 5$ & $\mathrm{RV}$ & Rating & RV & Rating & $\mathrm{RV}$ & Rating & RV & Rating & $\mathrm{RV}$ & Rating \\
\hline 1 & 3.6 & 5 & 0.733 & 3 & 0.849 & 4 & 0.699 & 4 & 0.753 & 4 & 0.766 & 3 \\
\hline 5 & 1.8 & 5 & 0.818 & 1 & 0.83 & 4 & 0.847 & 2 & 0.845 & 0 & 0.795 & 2 \\
\hline 8 & 2.4 & 5 & 1 & 0 & 1 & 2 & 0.68 & 4 & 0.81 & 2 & 0.75 & 4 \\
\hline 10 & 3.0 & 5 & 0.8 & 2 & 0.84 & 4 & 0.87 & 2 & 0.772 & 4 & 0.761 & 3 \\
\hline 16 & 2.8 & 5 & 0.74 & 3 & 0.96 & 2 & 0.61 & 3 & 0.794 & 2 & 0.741 & 4 \\
\hline 23 & 2.0 & 5 & 0.656 & 0 & 1.05 & 1 & 0.889 & 2 & 0.727 & 3 & 0.745 & $\overline{4}$ \\
\hline 25 & 2.6 & 5 & 0.82 & 1 & 1.03 & 1 & 0.655 & 4 & 0.73 & 3 & 0.734 & 4 \\
\hline 26 & 4.0 & 2 & - & - & -- & -- & 0.65 & 4 & - & -- & 0.73 & 4 \\
\hline 30 & 3.5 & 2 & - & - & - & -- & 0.68 & 4 & - & -- & 0.72 & 3 \\
\hline 33 & 2.7 & 3 & 0.757 & 4 & -. & -. & 0.71 & 4 & -- & - & 0.6 & 0 \\
\hline 38 & 3.6 & 5 & 0.76 & $\overline{4}$ & 0.83 & 4 & 0.871 & 2 & 0.756 & 4 & 0.749 & 4 \\
\hline 42 & 2.0 & 3 & $=$ & - & -- & - & 0.581 & 2 & 0.744 & 4 & 0,632 & 0 \\
\hline 45 & 2.5 & 4 & 0.709 & 2 & -- & -- & 0.665 & 4 & 0.809 & 2 & 0.701 & 2 \\
\hline 46 & 3.4 & 5 & 0.75 & 4 & 0.988 & 2 & 0.742 & 4 & 0.78 & 3 & 0.728 & 4 \\
\hline 59 & 3.4 & 5 & 0.755 & 4 & 0.78 & 3 & 0.702 & 4 & 0.741 & 4 & 0.7 & 2 \\
\hline 64 & 3.3 & 4 & 0.74 & 3 & - & - & 0.89 & 2 & 0.77 & 4 & 0.73 & 4 \\
\hline 70 & 2.4 & 5 & 0.622 & 0 & 0.838 & 4 & 0.694 & 4 & 0.944 & 0 & 0.744 & 4 \\
\hline 72 & 3.2 & 5 & 0.574 & 0 & 0.784 & 4 & 0.72 & 4 & 0.753 & 4 & 0.736 & 4 \\
\hline 80 & 2.0 & 3 & 0.9 & 0 & - & - & 0.8 & 3 & 0.79 & 3 & - & - \\
\hline 85 & 3.8 & 5 & 0.782 & 3 & 0.82 & 4 & 0.72 & 4 & 0.754 & 4 & 0.752 & 4 \\
\hline 86 & 2.3 & 3 & 0.693 & 1 & $=$ & - & 0.868 & 2 & 0.746 & 4 & - & $\mu$ \\
\hline 89 & 3.0 & 5 & 0.751 & 4 & 0.897 & 3 & 0.906 & 1 & 0.737 & 4 & 0.765 & 3 \\
\hline 97 & 3.2 & 5 & 0.792 & 3 & 0.82 & 4 & 0.717 & 4 & 0.78 & 3 & 0.784 & 2 \\
\hline 102 & 3.2 & 5 & 0.79 & 3 & 0.752 & 3 & 0.713 & 4 & 0.755 & 4 & 0.7 & 2 \\
\hline 105 & 2.0 & 5 & 0.76 & 4 & 1.02 & 1 & 0.93 & 1 & 0.729 & 3 & 0.684 & 1 \\
\hline 113 & 4.0 & 5 & 0.763 & 4 & 0.85 & 4 & 0.711 & 4 & 0.745 & 4 & 0.724 & 4 \\
\hline 118 & 3.0 & 3 & 0.777 & 4 & -- & -. & 0.905 & 1 & -- & $\cdots$ & 0.746 & 4 \\
\hline 134 & 2.8 & 5 & 0.79 & 3 & 0.833 & 4 & 0.91 & 1 & 0.775 & 3 & 0.762 & 3 \\
\hline 138 & 3.6 & 5 & 0.738 & 3. & 0.85 & 4 & 0.699 & 4 & 0.772 & 4 & 0.771 & 3 \\
\hline 142 & 2.8 & 5 & 0.704 & 2 & 0.855 & 4 & 0.885 & 2 & 0.741 & 4 & 0.795 & 2 \\
\hline 146 & 3.4 & 5 & 0.78 & 3 & 0.784 & 4 & 0.654 & 4 & 0.708 & 2 & 0.728 & 4 \\
\hline 180 & 3.6 & 5 & 0.763 & 4 & 0.805 & 4 & 0.665 & 4 & 0.74 & 4 & 0.784 & 2 \\
\hline 183 & 3.0 & 2 & $\rightarrow$ & - & -- & - & $<1$ & NR & 0.738 & 4 & 0.686 & 2 \\
\hline 190 & 3.2 & 5 & 0.694 & 1 & 0.754 & 3 & 0.731 & 4 & 0.747 & 4 & $0: 734$ & 4 \\
\hline 193 & 3.0 & 4 & 0.743 & 4 & 0.929 & 3 & 0.882 & 2 & 0.723 & 3 & - & - \\
\hline 198 & 2.0 & 4 & 0.824 & $\overline{1}$ & - & $\omega$ & 0.696 & 4 & 0.692 & 1 & 0.69 & 2 \\
\hline 205 & 4.0 & 2 & 0.777 & 4 & - & -- & 0.734 & 4 & - & - & - & - \\
\hline 208 & 2.0 & 2 & - & - & -. & -- & 0.69 & 4 & -- & -- & 0.58 & 0 \\
\hline 212 & 1.8 & 4 & 0,73 & 3 & 0.58 & 0 & 0.71 & 4 & - & - & 1 & 0 \\
\hline 224 & 2.4 & 5 & 0.834 & 1 & 0.762 & 3 & 0.72 & 4 & 0.536 & 0 & 0.753 & 4 \\
\hline 234 & 2.3 & 4 & 0.77 & 4 & - & - & 0.649 & 4 & 0.825 & 1 & 0.626 & 0 \\
\hline 247 & 3.6 & 5 & 0.75 & 4 & 0.79 & 4 & 0.65 & 4 & 0.79 & 3 & 0.77 & 3 \\
\hline 305 & 3.7 & 3 & 0.774 & 4 & -- & - & 0.71 & 4 & -- & - & 0.705 & 3 \\
\hline 306 & 1.5 & 4 & 0.731 & 3 & 1.45 & 0 & 1.04 & 0 & 0.721 & 3 & - & - \\
\hline 313 & 4.0 & 5 & 0.746 & 4 & 0.823 & 4 & 0.679 & 4 & 0.763 & 4 & 0.759 & 4 \\
\hline
\end{tabular}


Table 8. Laboratory performance ratings for standard reference sample $\mathbf{N}-74$ (nutrient constituents) -- continued [MPV, most probable value; Lab, laboratory number; OLR, overall laboratory rating for all rated analyses; $\mathrm{mg} / \mathrm{L}$, milligrams per liter; V/5, number of rated analyses out of 5 possible; RV, reported value; <, less than; NR, not rated; --, not reported.]

\begin{tabular}{|llll|}
\hline Rating & Absolute Z-value & Rating & Absolute Z-value \\
4 (Excellent) & $0.00-0.50$ & 1 (Marginal) & $1.51-2.00$ \\
3 (Good) & $0.51-1.00$ & 0 (Unsatisfactory) & greater than 2.00 \\
2 (Satisfactory) & $1.01-1.50$ & NR (Not Rated) & \\
\hline
\end{tabular}

\begin{tabular}{|c|c|c|c|c|c|c|c|c|c|c|c|c|}
\hline \multirow[b]{2}{*}{ Lab } & \multicolumn{2}{|c|}{$\begin{array}{r}\text { Analyte }= \\
\text { MPV }= \\
\text { F-pseudosigma }=\end{array}$} & \multicolumn{2}{|c|}{$\begin{array}{l}\text { Ammonla as } \mathrm{N} \\
0.760 \mathrm{mg} / \\
0.037\end{array}$} & \multicolumn{2}{|c|}{$\begin{array}{l}\text { Ammonia + } \\
\text { Organic } \mathrm{N} \text { as } \mathrm{N} \\
0.838 \mathrm{mg} / \mathrm{L} \\
0.113\end{array}$} & \multicolumn{2}{|c|}{$\begin{array}{l}\text { Nituate as } \mathrm{N} \\
0.711 \mathrm{mg} / \mathrm{L} \\
0.122\end{array}$} & \multicolumn{2}{|c|}{$\begin{array}{c}\text { Phosphorus as P } \\
0.755 \mathrm{mg} / \mathrm{L} \\
0.031\end{array}$} & \multicolumn{2}{|c|}{$\begin{array}{c}\text { Orthophosphate } \\
\text { as P } \\
0.741 \mathrm{mg} / \\
0.035\end{array}$} \\
\hline & OLA & $\mathrm{V} / 5$ & $\mathrm{RV}$ & Rating & RV & Rating & RV & Rating & RV & Rating & RV & Rating \\
\hline 320 & 3.4 & 5 & 0.792 & 3 & 0.899 & 3 & 0.697 & 4 & 0.773 & 4 & 0.715 & 3 \\
\hline 328 & 1.2 & 5 & 0.84 & 0 & 1.53 & 0 & 0.85 & 2 & 0.76 & 4 & 2.33 & 0 \\
\hline 341 & 3.0 & 5 & 0.766 & 4 & 0.84 & 4 & 0.69 & 4 & 0.776 & 3 & 0.65 & 0 \\
\hline 356 & 3.0 & 5 & 0.68 & 0 & 0.795 & 4 & 0.714 & 4 & 0.784 & 3 & 0.754 & 4 \\
\hline 366 & 2.6 & 5 & 0.74 & 3 & 0.61 & 0 & 0.711 & 4 & 0.702 & 2 & 0.733 & 4 \\
\hline 370 & 2.4 & 5 & 0.9 & 0 & 0.7 & 2 & 0.715 & 4 & 0.73 & 3 & 0.775 & 3 \\
\hline 372 & 2.4 & 5 & 0.758 & 4 & 1.97 & 0 & 0.682 & 4 & 1.05 & 0 & 0.75 & 4 \\
\hline 373 & 3.2 & 5 & 0.7745 & 4 & 1.211 & 0 & 0.705 & 4 & 0.762 & 4 & 0.726 & 4 \\
\hline 376 & 3.6 & 5 & 0.758 & 4 & 0.828 & 4 & 0.672 & 4 & 0.751 & 4 & 0.791 & 2 \\
\hline
\end{tabular}


Table 9. Laboratory performance ratings for standard reference sample P-38 (low-ionic strength constituents)

[MPV, most probable value; Lab, laboratory identification number; OLR, overall laboratory rating for all rated analyses; $\mathrm{mg} / \mathrm{L}$, milligrams per liter; $\mu \mathrm{S} / \mathrm{cm}$, microsiemens per centimeter at 25 degrees Celsius; V/11, number of rated analyses out of 11 possible; RV, reported value; <, less than; NR, not rated; --, not reported.]

\begin{tabular}{|lcll|}
\hline Rating & Absolute Z-value & Rating & Absolute Z-value \\
4 (Excellent) & $0.00-0.50$ & 1 (Marginal) & $1.51-2.00$ \\
3 (Good) & $0.51-1.00$ & 0 (Unsatisfactory) & greater than 2.00 \\
2 (Satisfactory) & $1.01-1.50$ & NR (Not Rated) & \\
\hline
\end{tabular}

\begin{tabular}{|c|c|c|c|c|c|c|c|c|c|c|c|c|}
\hline \multirow[b]{2}{*}{ Lab } & \multicolumn{2}{|c|}{$\begin{array}{r}\text { Analyte }= \\
M P V= \\
\text { F-pseudosigma }=\end{array}$} & \multicolumn{2}{|c|}{$\begin{array}{c}\text { Acldity } \\
\text { inadequate data }\end{array}$} & \multicolumn{2}{|c|}{$\begin{array}{l}\text { Calclum } \\
6.40 \mathrm{mg} / \mathrm{L} \\
0.33\end{array}$} & \multicolumn{2}{|c|}{$\begin{array}{l}\text { Chlorlde } \\
8.37 \mathrm{mg} / \mathrm{L} \\
0.39\end{array}$} & \multicolumn{2}{|c|}{$\begin{array}{c}\text { Fluorlde } \\
\text { inadequate data }\end{array}$} & \multicolumn{2}{|c|}{$\begin{array}{l}\text { Potassium } \\
0.830 \mathrm{mg} / \\
0.081\end{array}$} \\
\hline & OLR & $V / 11$ & RV & Rating & RV & Rating & RV & Rating & RV & Rating & $\mathrm{AV}$ & Rating \\
\hline 1 & 3.1 & 7 & - & $\ldots$ & 6.2 & 3 & 7.91 & 2 & 0.026 & NR & 0.79 & 4 \\
\hline 2 & 3.6 & 7 & - & - & 6.472 & 4 & - & - & 0.037 & NR & 0.854 & 4 \\
\hline 5 & 1.3 & 8 & - & -.. & 15.7 & 0 & 8.39 & 4 & -. & -- & 3.38 & 0 \\
\hline 8 & 2.3 & 8 & - & - & 6.8 & 2 & 8.35 & 4 & $<0.3$ & NR & 0.85 & 4 \\
\hline 23 & 2.7 & 7 & - & - & 6.22 & 3 & 8.7 & 3 & $<0.15$ & NR & 0.97 & 1 \\
\hline 25 & 2.6 & 7 & 14 & NR & 5.92 & 2 & 8.4 & 4 & 0.04 & NR & 0.99 & 1 \\
\hline 33 & 1.4 & 8 & - & - & 5.95 & 2 & 8.55 & 4 & 0.022 & NR & 0.64 & 0 \\
\hline 38 & 3.5 & 6 & 2.96 & NR & 6.4 & 4 & - & - & -- & -- & 0.8 & 4 \\
\hline 45 & 3.5 & 8 & - & - & 6.35 & 4 & 8.15 & 3 & 0.05 & NR & 0.819 & 4 \\
\hline 59 & 1.4 & 8 & - & - & 7.48 & 0 & 7.73 & 1 & 0.28 & NR & 0.54 & 0 \\
\hline 64 & 3.1 & 8 & $m$ & $=$ & 6.51 & 4 & 8.78 & 3 & - & -- & 0.69 & 1 \\
\hline 85 & 3.3 & 8 & - & 2. & 6.77 & 2 & 8.5 & 4 & 0.03 & NR & 0.9 & 3 \\
\hline 86 & 3.1 & 8 & - & -. & 6.27 & 4 & 8.98 & 2 & 0.22 & NR & 0.83 & 4 \\
\hline 89 & 2.5 & 8 & 2.8 & NR & 6.88 & 2 & 8.56 & 4 & $<0.1$ & NR & 0.865 & 4 \\
\hline 105 & 3.3 & 7 & 6.8 & NR & 6.57 & 3 & 8 & 3 & $<0.20$ & NR & $<1.00$ & NR \\
\hline 110 & 3.6 & 8 & - & - & 6.4034 & 4 & 8.168 & 4 & $=$ & -- & 0.8389 & 4 \\
\hline 113 & 3.9 & 7 & - & - & 6.28 & 4 & 8.32 & 4 & -- & -- & 0.789 & 4 \\
\hline 134 & 3.8 & 8 & - & - & 6.5 & 4 & 8.08 & 3 & $<0.1$ & NR & 0.809 & 4 \\
\hline 138 & 3.8 & 8 & - & - & 6.3 & 4 & 8.91 & 2 & $<0.050$ & NR & 0.79 & 4 \\
\hline 180 & 2.9 & 8 & - & - & 6.37 & 4 & 8.08 & 3 & 0.012 & NR & 0.747 & 2 \\
\hline 190 & 3.4 & 8 & - & -2 & 6.21 & 3 & 8.52 & 4 & 0.01 & NR & 0.78 & 3 \\
\hline 193 & 3.7 & 6 & - & - & 5.97 & 2 & - & - & -- & - & 0.839 & 4 \\
\hline 208 & 4.0 & 1 & - & - & -- & - & 8.31 & 4 & -- & -- & - & - \\
\hline 245 & 3.7 & 3 & - & - & 6.447 & 4 & - & - & - & -- & 0.7702 & 3 \\
\hline 247 & 2.1 & 8 & 5 & NA & 5.69 & 0 & 7.98 & 3 & 0.02 & NR & 0.72 & 2 \\
\hline 265 & 3.5 & 6 & $m$ & 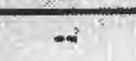 & 6.5 & 4 & 7.7 & 1 & -- & - & 0.83 & $\overline{4}$ \\
\hline 279 & 1.8 & 4 & $\cdots$ & - & 7.37 & 0 & - & - & -- & -. & 0.86 & 4 \\
\hline 315 & 3.2 & 6 & - & -. & 6.3 & 4 & 8.3 & 4 & - & -. & 0.97 & 1 \\
\hline 321 & 1.9 & 8 & - & - & 6.26 & 4 & 8.44 & 4 & -- & - & 0.75 & 3 \\
\hline 328 & 2.4 & 8 & 4.5 & NR & 6.7 & 3 & 9 & 2 & 0.026 & NR & 1.63 & 0 \\
\hline 333 & 3.6 & 7 & $-m$ & - & 6.52 & 4 & 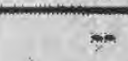 & - & .. & -- & 0.87 & 4 \\
\hline 336 & 0.4 & 8 & - & - & 16.12 & 0 & 29.82 & 0 & 0.93 & NR & 3.419 & 0 \\
\hline 370 & 1.9 & 8 & 20 & NA & 6.89 & 2 & 8.71 & 3 & 0.062 & NR & 1.05 & 0 \\
\hline 372 & 1.6 & 8 & 18.2 & NR & 6.2 & 3 & 8.14 & 3 & 0.1 & NR & 0.39 & 0 \\
\hline
\end{tabular}


Table 9. Laboratory performance ratings for standard reference sampie P-38 (iow-ionic strength constituents)

- continued

[MPV, most probable value; Lab, laboratory identification number; OLR, overall laboratory rating for all rated analyses; $\mathrm{mg} / \mathrm{L}, \mathrm{milligrams}$ per liter; $\mu \mathrm{S} / \mathrm{cm}$, microsiemens per centimeter at 25 degrees Celsius; V/11, number of rated analyses out of 11 possible; RV, reported value; <, less than; NR, not rated; --, not reported.]

\begin{tabular}{|lcll|}
\hline Rating & Absolute Z-value & Rating & $\frac{\text { Absolute Z-value }}{1.51-2.00}$ \\
4 (Excellent) & $0.00-0.50$ & 1 (Marginal) & greater than 2.00 \\
3 (Good) & $0.51-1.00$ & 0 (Unsatisfactory) & \\
2 (Satisfactory) & $1.01-1.50$ & NR (Not Rated) & \\
\hline
\end{tabular}

\begin{tabular}{|c|c|c|c|c|c|c|c|c|c|c|c|}
\hline & $\begin{array}{r}\text { Analyte }= \\
M P V= \\
\text { F-pseudosigma }= \\
\end{array}$ & $\begin{array}{l}\text { Magne } \\
1.15 \\
0.05 \\
\end{array}$ & $\begin{array}{l}\text { lum } \\
\text { grh. }\end{array}$ & $\begin{array}{c}\text { Sod } \\
1.80 \\
0.12 \\
\end{array}$ & um & $\begin{array}{l}\text { pl } \\
6.01 \\
0.21 \\
\end{array}$ & & $\begin{array}{r}\text { Orthoph } \\
\text { as } \\
\text { inadequ }\end{array}$ & $\begin{array}{l}\text { sphate } \\
\text { te data }\end{array}$ & $\begin{array}{l}\text { Sulf } \\
2.99 \\
0.21 \\
\end{array}$ & ite \\
\hline Lab & & AV & Rating & RV & Rating & RV & Rating & RV & Rating & RV & Rating \\
\hline 1 & & 1.17 & 4 & 1.78 & 4 & - & - & - & $\cdots$ & 2.75 & 2 \\
\hline 2 & & 1.158 & 4 & 1.915 & 3 & 6.034 & 4 & - & -- & 3.086 & 4 \\
\hline 5 & & 5.67 & 0 & 24 & 0 & 5.66 & 2 & - & -- & 2.87 & 3 \\
\hline 8 & & 1.2 & 3 & 1.9 & 3 & 6.73 & 0 & $<0.2$ & NR & 3.21 & 2 \\
\hline 23 & & 1.11 & 3 & 1.76 & 4 & 6.32 & 2 & 0.001 & NR & $<5.0$ & NR \\
\hline 25 & & 1.09 & 2 & 1.65 & 2 & 6.17 & 3 & 0.03 & NR & $<5.0^{\circ}$ & NR \\
\hline 33 & & 0.795 & 0 & 0.676 & 0 & 6.21 & 3 & 0.139 & NR & 3.24 & 2 \\
\hline 38 & & 1.081 & 2 & 1.74 & 3 & 6.1 & 4 & -- & -- & .. & - \\
\hline 45 & & 1.16 & 4 & 1.85 & 4 & 5.96 & 4 & $<0.1$ & NR & 2.98 & 4 \\
\hline 59 & & 0.96 & 0 & 2 & 1 & 5.99 & 4 & - & -- & 2.78 & 3 \\
\hline 64 & & 1.13 & 4 & 1.74 & 3 & 6.07 & $\overline{4}$ & $<0.002$ & NR & 3.11 & $\overline{3}$ \\
\hline 85 & & 1.18 & 3 & 1.96 & 2 & 6.13 & 4 & $<0.001$ & NR & 2.9 & 4 \\
\hline 86 & & 1.17 & 4 & 1.85 & 4 & 5.9 & 4 & -- & .- & 4.27 & 0 \\
\hline 89 & & 1.36 & 0 & 2.04 & 0 & 5.7 & 2 & $<0.003$ & NR & 2.95 & 4 \\
\hline 105 & & 1.19 & 3 & 1.93 & 2 & 5.9 & 4 & 0.01 & NR & 3.01 & 4 \\
\hline 110 & & 1.1124 & 3 & 1.768 & 4 & 5.935 & 4 & $=$ & - & 3.006 & 4 \\
\hline 113 & & 1.1 & 3 & 1.76 & 4 & - & - & $<0.004$ & NR & 2.9 & 4 \\
\hline 134 & & 1,13 & 4 & 1.72 & 3 & 5.88 & 4 & $<0.01$ & NR & 2.99 & 4 \\
\hline 138 & & 1.16 & 4 & 1.8 & 4 & 6.05 & 4 & $<0.004$ & NR & 2.94 & 4 \\
\hline 180 & & 1.13 & 4 & 1.78 & 4 & 6.05 & 4 & $<0.005$ & NR & 2.74 & 2 \\
\hline 190 & & 1.16 & 4 & 1.79 & 4 & 5.6 & 2 & - & - & 2.95 & 4 \\
\hline 193 & & 1.16 & 4 & 1.8 & 4 & - & - & - & -- & 2.96 & 4 \\
\hline 208 & & - & - & -. & .- & $\cdots$ & - & -- & -- & $<3$ & NR \\
\hline 245 & & 1.122 & 4 & - & -- & - & - & -- & -. & - & - \\
\hline 247 & & 0.95 & 0 & 1.65 & 2 & 6.22 & 3 & $<0.01$ & NR & 2.84 & 3 \\
\hline 265 & & 1,15 & $\overline{4}$ & 1.85 & 4 & 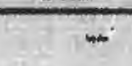 & - & - & -- & 2.9 & 4 \\
\hline 279 & & 1,34 & 0 & 1.87 & 3 & .. & - & - & -- & -. & - \\
\hline 315 & & 1.2 & 3 & 1.8 & 4 & - & - & - & -. & 3.1 & 3 \\
\hline 321 & & 1.06 & 1 & 1.54 & 0 & 5.7 & 2 & $<0.001$ & NR & 2.66 & 1 \\
\hline 328 & & 1.18 & 4 & 1.8 & 4 & 5.6 & 2 & 0.037 & NR & 4.44 & 0. \\
\hline$\overline{333}$ & & 1.17 & 4 & 1.9 & 3 & 5.92 & 4 & - & - & 3.17 & 3 \\
\hline 336 & & 4.34 & 0 & 25.5 & 0 & 6.23 & 3 & - & -- & 10.86 & 0 \\
\hline 370 & & 1.06 & 1 & 1.92 & 2 & 5.77 & 3 & $<0.02$ & NR & 3.32 & 1 \\
\hline 372 & & 1.06 & 1. & 1.07 & 0 & 6.36 & 2 & $<0.01$ & NR & 3.95 & 0 \\
\hline
\end{tabular}


Table 9. Laboratory performance ratings for standard reference sample P-38 (low-ionic strength constituents) - continued

[MPV, most probable value; Lab, laboratory identification number; OLR, overall laboratory rating for all rated analyses; mg/L, milligrams per liter; $\mu \mathrm{S} / \mathrm{cm}$, microsiemens per centimeter at 25 degrees Celsius; V/11, number of rated analyses out of 11 possible; RV, reported value; <, less than; NR, not rated; --, not reported.]

\begin{tabular}{|lcll|}
\hline Rating & Absolute Z-value & Rating & Absolute Z-value \\
4 (Excellent) & $0.00-0.50$ & 1 (Marginal) & $1.51-2.00$ \\
3 (Good) & $0.51-1.00$ & 0 (Unsatisfactory) & greater than 2.00 \\
2 (Satisfactory) & $1.01-1.50$ & NR (Not Rated) & \\
\hline
\end{tabular}

\begin{tabular}{|c|c|c|c|}
\hline & $\begin{array}{r}\text { Analyte }= \\
\text { MPV }= \\
\text { F-pseudosigma }=\end{array}$ & $\begin{array}{r}\text { Spec } \\
\text { Conduc } \\
65.01 \\
3.3\end{array}$ & $\begin{array}{l}\text { fic } \\
\text { tance } \\
\text { S/cm }\end{array}$ \\
\hline $\mathrm{Lab}$ & & RV & Rating \\
\hline 1 & & 66.9 & 3 \\
\hline 2 & & 60.267 & 2 \\
\hline 5 & & 58.6 & 1 \\
\hline 8 & & 57.4 & 0 \\
\hline 23 & & 67.7 & 3 \\
\hline 25 & & 64 & 4 \\
\hline 33 & & 72.8 & 0 \\
\hline 38 & & 63.8 & 4 \\
\hline 45 & & 70 & 1 \\
\hline 59 & & 69 & 2 \\
\hline 64 & & 62.5 & 3 \\
\hline 85 & & 66 & 4 \\
\hline 86 & & 66.9 & 3 \\
\hline 89 & & 64.6 & 4 \\
\hline 105 & & 66.6 & 4 \\
\hline 110 & & 61.5 & 2 \\
\hline 113 & & 66.3 & 4 \\
\hline 134 & & 64.1 & 4 \\
\hline 138 & & 64.9 & 4 \\
\hline 180 & & 45 & 0 \\
\hline 190 & & 63.2 & 3 \\
\hline 193 & & 65 & 4 \\
\hline 208 & & - & n- \\
\hline 245 & & - & - \\
\hline 247 & & 65 & 4 \\
\hline 265 & & - & - \\
\hline 279 & & - & - \\
\hline 315 & & - & - \\
\hline 321 & & 57.9 & 0 \\
\hline 328 & & 65 & 4 \\
\hline 333 & & 61.8 & 3 \\
\hline 336 & & 244.6 & 0 \\
\hline 370 & & 68 & 3 \\
\hline 372 & & 65 & 4. \\
\hline
\end{tabular}


Table 10. Laboratory performance ratings for standard reference sample HG-34 (Mercury)

[MPV, most probable value; Lab, laboratory identification number; OLR, overall laboratory rating for all rated analyses; $\mu \mathrm{g} / \mathrm{L}$, micrograms per liter; V/1, number of rated analyses out of 1 possible; RV, reported value; <, less than; NR, not rated; --, not reported.]

\begin{tabular}{|lcll|}
\hline Rating & Absolute Z-value & Rating & Absolute Z-value \\
4 (Excellent) & $0.00-0.50$ & 1 (Marginal) & $1.51-2.00$ \\
3 (Good) & $0.51-1.00$ & 0 (Unsatisfactory) & greater than 2.00 \\
2 (Satisfactory) & $1.01-1.50$ & NR (Not Rated) & \\
\hline
\end{tabular}

\begin{tabular}{|c|c|c|c|c|}
\hline \multirow[b]{2}{*}{ Lab } & \multicolumn{2}{|c|}{$\begin{array}{r}\text { Analyte }= \\
M P V= \\
\text { F-pseudosigma }=\end{array}$} & \multicolumn{2}{|c|}{$\begin{array}{c}\text { Mercury } \\
\text { Inadequate data }\end{array}$} \\
\hline & OLR & $\mathrm{V} / 1$ & RV & Rating \\
\hline 1 & NR & 0 & 0.0266 & NR \\
\hline 5 & NR & 0 & 1.63 & NR \\
\hline 8 & NR & 0 & 0.15 & NR \\
\hline 23 & NR & 0 & 0.1 & NP \\
\hline 26 & NR & 0 & $<0.2$ & NA \\
\hline 32 & NR & 0 & $<0,05$ & NR \\
\hline 45 & NR & 0 & 0.0319 & NA \\
\hline 46 & NR & 0 & 0.011 & NA \\
\hline 59 & NR & 0 & 0.025 & NR \\
\hline 89 & NR & 0 & $<0.2$ & NA \\
\hline 97 & NR & 0 & $<0.1$ & NR \\
\hline 105 & NR & 0 & 1.13 & NA \\
\hline 134 & NR & 0 & $<0.1$ & NR \\
\hline 138 & NR & 0 & 0.028 & NR \\
\hline 147 & NR & 0 & 0.0385 & NR \\
\hline 180 & NR & 0 & $<0.050$ & NA \\
\hline 193 & NR & 0 & 0.0249 & NR \\
\hline 245 & NR & 0 & 0.0248 & NR \\
\hline 247 & NR & 0 & $<0.20$ & NR \\
\hline 304 & NR & 0 & 0.028 & NR \\
\hline 356 & NR & 0 & 0.011 & NR \\
\hline 370 & NR & 0 & $<100$ & NR \\
\hline 372 & NR & 0 & $<0.0002$ & NR \\
\hline
\end{tabular}


Table 11. Statistical summary of reported dsta for stsndard reference sample T-169 (trace constituents)

T-169 SILVER (Ag) in $\mu g / L$

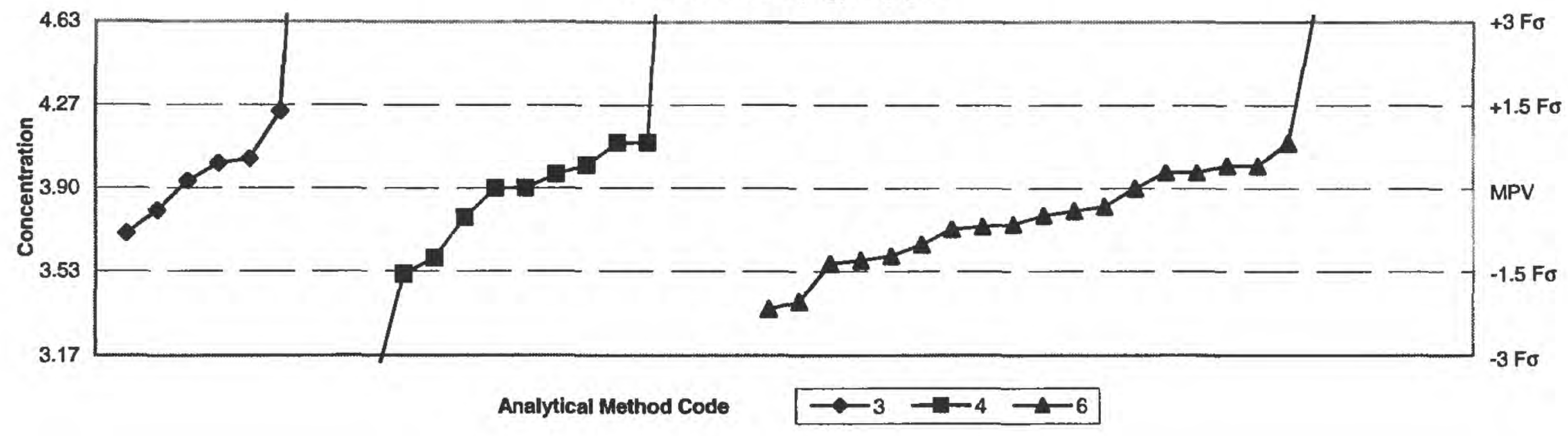

\begin{tabular}{|c|c|c|c|c|c|c|}
\hline \multirow[t]{2}{*}{ SUMMARY } & \multicolumn{4}{|c|}{ Methods } & \multicolumn{2}{|c|}{ Statistics } \\
\hline & 3 & 4 & 6 & Method Codes & & \\
\hline$n=$ & $\overline{7}$ & 13 & 19 & 03 Atomic absorption: graphite furnace & MPV $=$ & $3.90 \mu g / L$ \\
\hline Minimum $=$ & 3.7 & 2.45 & 3.37 & 04 Inductively coupled plasma & F.pseudosigma $=$ & 0.24 \\
\hline Maximum $=$ & 6.47 & 6.6 & 4.8 & 06 Inductively coupled plasma/mass spectrometry & $\mathrm{n}=$ & 39 \\
\hline Medlan $=$ & 4.01 & 3.90 & 3.78 & & Uh $=$ & 4.01 \\
\hline F-pseudosigma = & 0.20 & 0.38 & 0.26 & & $\operatorname{Lh}=$ & 3.68 \\
\hline
\end{tabular}

\begin{tabular}{|c|c|c|c|c|c|c|c|c|c|c|c|}
\hline \multicolumn{9}{|c|}{ Method Codes } & \multirow{2}{*}{\multicolumn{3}{|c|}{$6^{\text {Method Codes }}$}} \\
\hline Lab & Rating & Z-value & 3 & 4 & 6 & Lab & Rating & Z-value & & & \\
\hline 1 & 4 & 0.29 & - & - & 3.97 & 370 & 0 & 10.51 & 6.47 & - & - \\
\hline 5 & 0 & 10.02 & -. & 6.35 & - & 372 & 0 & -3.68 & -. & 3 & -. \\
\hline 7 & 0 & -7.77 & - & -- & $<2$ & & & & & & \\
\hline 8 & 2 & -1.02 & - & -- & 3.65 & & & & & & \\
\hline 12 & 3 & -0.82 & 3.7 & - & -. & & & & & & \\
\hline 16 & 4 & 0.41 & - & 4 & - & & & & & & \\
\hline 23 & 1 & -1.55 & .. & 3.52 & - & & & & & & \\
\hline 25 & NR & -- & -- & $<17$ & - & & & & & & \\
\hline 32 & 4 & 0.00 & - & - & 3.9 & & & & & & \\
\hline 42 & 2 & -1.23 & - & - & 3.6 & & & & & & \\
\hline 45 & 0 & 3.68 & 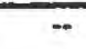 & - & 4.8 & & & & & & \\
\hline 59 & NR & - & - & -- & $<5$ & & & & & & \\
\hline 86 & 0 & -5.93 & - & 2.45 & -- & & & & & & \\
\hline 89 & 4 & -0.41 & 3.8 & -- & -- & & & & & & \\
\hline 97 & 4 & 0.45 & 4.01 & - & -. & & & & & & \\
\hline 105 & 2 & -1.31 & - & $=$ & 3.58 & & & & & & \\
\hline 113 & 3 & 0.53 & 4.03 & - & -- & & & & & & \\
\hline 121 & 0 & -2.04 & - & - & 3.4 & & & & & & \\
\hline 134 & 4 & 0.00 & - & 3.9 & -- & & & & & & \\
\hline 138 & 4 & -0.33 & - & -. & 3.82 & & & & & & \\
\hline 142 & 3 & -0.74 & - & - & 3.72 & & & & & & \\
\hline 146 & 2 & -1.27 & - & 3.59 & -- & & & & & & \\
\hline 149 & 3 & 0.82 & - & -- & 4.1 & & & & & & \\
\hline 180 & 0 & -2.17 & -. & - & 3.37 & & & & & & \\
\hline 190 & 4 & 0.12 & 3.93 & $\cdots$ & -. & & & & & & \\
\hline 193 & 2 & 1.39 & 4.24 & $\cdots$ & - & & & & & & \\
\hline 198 & 3 & -0.69 & - & -- & 3.73 & & & & & & \\
\hline 212 & 3 & 0.82 & - & 4.1 & - & & & & & & \\
\hline 234 & 3 & -0.53 & - & 3.77 & -- & & & & & & \\
\hline 245 & 2 & -1.36 & -. & .- & 3.568 & & & & & & \\
\hline 247 & 4 & -0.49 & - & $=$ & 3.78 & & & & & & \\
\hline 255 & 4 & 0.41 & - & - & 4 & & & & & & \\
\hline 256 & 4 & 0.25 & - & 3.96 & - & & & & & & \\
\hline 259 & 4 & 0.00 & $\cdots$ & 3.9 & -- & & & & & & \\
\hline 265 & 4 & -0.41 & - & -. & 3.8 & & & & & & \\
\hline 304 & 4 & 0.29 & - & 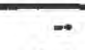 & 3.97 & & & & & & \\
\hline 305 & 3 & 0.82 & -- & 4.1 & - & & & & & & \\
\hline 328 & 0 & 11.04 & -. & 6.6 & -. & & & & & & \\
\hline 330 & 4 & 0.41 & - & -- & 4 & & & & & & \\
\hline 356 & 3 & -0.65 & 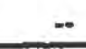 & - & 3.74 & & & & & & \\
\hline
\end{tabular}


Table 11. Statistical summary of reported data for standard reference sample T-169 (trace constituents) -- continued

T-169 ALUMINUM (Al) in $\mu \mathrm{g} / \mathrm{L}$

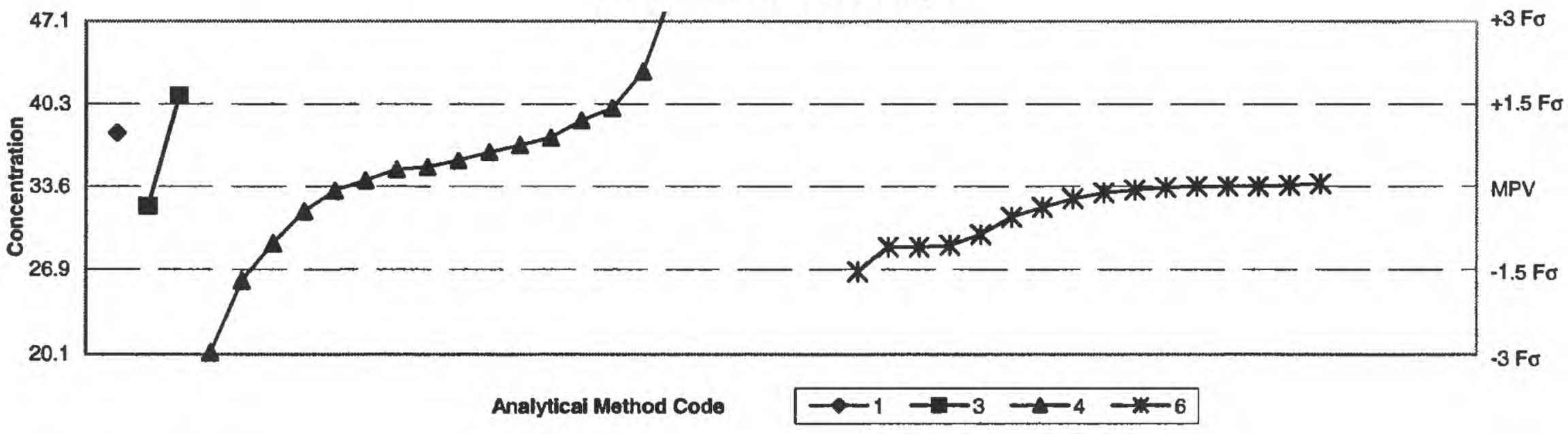

\begin{tabular}{|c|c|c|c|c|c|c|c|c|}
\hline \multirow[t]{2}{*}{ SUMMARY } & \multicolumn{6}{|c|}{ Methods } & \multicolumn{2}{|c|}{ Statistics } \\
\hline & 1 & 3 & 4 & 5 & 6 & Method Codes & & \\
\hline$n=$ & 1 & 2 & 19 & 1 & 16 & 01 Atomic absorption: direct, air & MPV $=$ & $33.6 \mu \mathrm{g} /$ \\
\hline Minimum $=$ & 38 & 32 & 20.3 & 53 & 26.7 & 03 Atomic absorption: graphite fumace & F-pseudosigma $=$ & 4.5 \\
\hline Maximum $=$ & & 41 & 290 & & 33.8 & 04 Inductively coupled plasma & $n=$ & 39 \\
\hline Median = & & & 36.4 & & 32.8 & 05 Direct current plasma & $U_{n}=$ & 37.8 \\
\hline F-pseudosigma $=$ & & & 5.8 & & 3.2 & 06 Inductively coupled plasma/mass sp & ometry & 31.8 \\
\hline
\end{tabular}

\begin{tabular}{|c|c|c|c|c|c|c|c|c|c|c|c|c|c|c|c|}
\hline \multicolumn{11}{|c|}{ Method Codes } & \multicolumn{5}{|c|}{ Method Codes } \\
\hline Lab & Rating & Z-value & 1 & 3 & 4 & 5 & 6 & Lab & Rating & Z-value & 1 & 3 & 4 & 5 & 6 \\
\hline 1 & 4 & -0.06 & $\cdots$ & 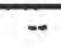 & $\cdots$ & $\cdots$ & 33.31 & 372 & 2 & 1.20 & - & - & 39 & -- & $\because$ \\
\hline 5 & 0 & 3.63 & -- & -. & 49.9 &.- & - & & & & & & & & \\
\hline 7 & 4 & -0.02 & - & -. & -- & -. & 33.5 & & & & & & & & \\
\hline 8 & 1 & -1.54 & - & -- & - & - & 26.7 & & & & & & & & \\
\hline 16 & 2 & -1.03 & $\cdots$ & - & 29 & -- & -. & & & & & & & & \\
\hline 25 & 0 & 57.17 & - & 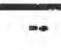 & 290 & - & -- & & & & & & & & \\
\hline 26 & 4 & 0.36 & - & - & 35.2 & - & - & & & & & & & & \\
\hline 32 & 3 & -0.56 & - & - & -- & - & 31.1 & & & & & & & & \\
\hline 33 & 0 & 4.33 & -- & -- & -- & 53 & -. & & & & & & & & \\
\hline 42 & 3 & -0.87 & - & -. & -. & -. & 29.7 & & & & & & & & \\
\hline 45 & 4 & 0.00 & 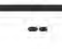 & 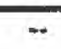 & - & $=$ & 33.6 & & & & & & & & \\
\hline 59 & NR & -. & - & -. & -. & .. & $<50$ & & & & & & & & \\
\hline 70 & 2 & -1.09 & - & - & -- & -- & 28.7 & & & & & & & & \\
\hline 76 & 4 & -0.12 & -- & - & -- & - & 33.08 & & & & & & & & \\
\hline 89 & 3 & 0.98 & 38 & .. & - & 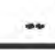 & -- & & & & & & & & \\
\hline 97 & 1 & 1.65 & - & $\overline{41}$ & - & - & - & & & & & & & & \\
\hline 105 & 4 & 0.02 & - & - & - & -. & 33.69 & & & & & & & & \\
\hline 110 & 4 & 0.11 & - & - & 34.1 & -. & -- & & & & & & & & \\
\hline 113 & 4 & -0.45 & - & -- & 31.6 & -. & .. & & & & & & & & \\
\hline 134 & 4 & -0.08 & - & -- & 33.25 & -- & -. & & & & & & & & \\
\hline 138 & 3 & 0.62 & - & 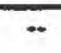 & 36.4 & - & - & & & & & & & & \\
\hline 142 & 3 & 0.89 & - & -- & 37.6 & - & .. & & & & & & & & \\
\hline 146 & 0 & 6.60 & - & -- & 63.2 & .. & -- & & & & & & & & \\
\hline 149 & 4 & 0.00 & - & $\cdots$ & -- & - & 33.6 & & & & & & & & \\
\hline 180 & 2 & -1.07 & - & - & $\cdots$ & $\cdots$ & 28.8 & & & & & & & & \\
\hline 190 & 4 & -0.36 & - & 32 & $=$ & - & -- & & & & & & & & \\
\hline 198 & 2 & -1.09 & - &.- & -- & .- & 28.7 & & & & & & & & \\
\hline 212 & 0 & -2.97 & - & -. & 20.3 & -- & -. & & & & & & & & \\
\hline 234 & 2 & 1.43 & - & -. & 40 & - & -- & & & & & & & & \\
\hline 245 & 4 & 0.04 & - & -. & - & - & 33.8 & & & & & & & & \\
\hline 247 & 4 & -0.22 & $\cdots$ & $\because$ & - & $=$ & 32.6 & & & & & & & & \\
\hline 256 & 4 & 0.48 & - & -. & 35.75 & - & .. & & & & & & & & \\
\hline 259 & 3 & 0.76 & - & .. & 37 & -. & .. & & & & & & & & \\
\hline 265 & 4 & 0.31 & -. & 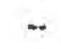 & 35 & -- & -- & & & & & & & & \\
\hline 304 & 4 & -0.38 & - & - & - & .. & 31.9 & & & & & & & & \\
\hline 305 & 0 & 4.10 & - & .. & 52 & - & $=$ & & & & & & & & \\
\hline 328 & 1 & -1.69 & $\cdots$ & -- & 26 & - & -. & & & & & & & & \\
\hline 330 & 4 & 0.00 & -- & - & - & - & 33.6 & & & & & & & & \\
\hline 358 & 0 & 2.10 & -. & - & 43 & -- & -- & & & & & & & & \\
\hline 370 & NR & - & 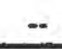 & - & $<100$ & .. & $\ldots$ & & & & & & & & \\
\hline
\end{tabular}


T-169 ARSENIC (As) in $\mu g / L$

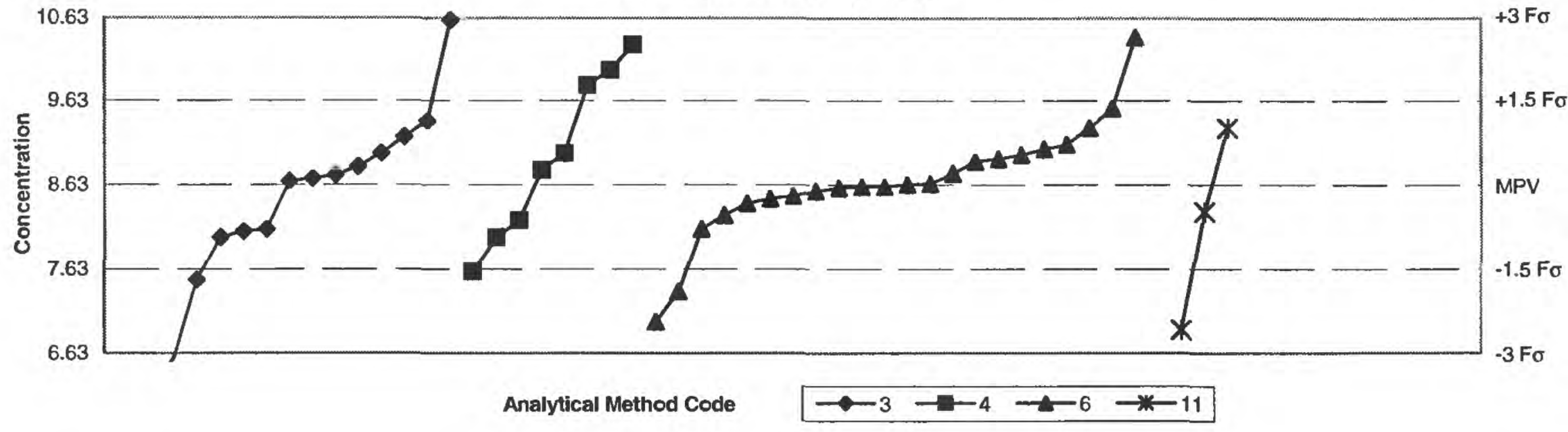

\begin{tabular}{|c|c|c|c|c|c|c|c|c|}
\hline \multirow[t]{2}{*}{ SUMMARY } & \multicolumn{6}{|c|}{ Methods } & \multicolumn{2}{|c|}{ Statistles } \\
\hline & 3 & 4 & 6 & 10 & 11 & Method Codes & & \\
\hline$n=$ & 15 & 8 & 22 & 1 & 3 & 03 Atomic absorption; graphite fumace & MPV $=$ & $8.63 \mu \mathrm{g} / \mathrm{L}$ \\
\hline Minimum = & 6.09 & 7.6 & 7 & 2.8 & 6.9 & 04 Inductively coupled plasma & F-pseudosigma $=$ & 0.67 \\
\hline Maximum $=$ & 10.6 & 10.31 & 10.4 & & 9.3 & 06 Inductively coupled plasma/mass spectrometry & $n=$ & 49 \\
\hline Median $=$ & 8.67 & 8.90 & 8.61 & & & 10 Atomic absorption: extraction & Uh $=$ & 9.00 \\
\hline F-pseudosigma = & 0.87 & 1.34 & 0.39 & & & 11 Atomic absorption: hydride & $\mathrm{Lh}=$ & 8.10 \\
\hline
\end{tabular}

\begin{tabular}{|c|c|c|c|c|c|c|c|c|c|c|c|c|c|c|c|}
\hline \multicolumn{11}{|c|}{ Method Codes } & \multicolumn{5}{|c|}{ Method Codes } \\
\hline Lab & Rating & Z-value & 3 & 4 & 6 & 10 & 11 & Lab & Rating & Z-value & 3 & 4 & 6 & 10 & 11 \\
\hline 1 & 3 & 0.56 & 9 & 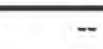 & $\cdots$ & - & $\ldots$ & 256 & 0 & -8.73 & - & $=$ & $\ldots$ & 2.8 & - \\
\hline 5 & 2 & 1.13 & 9.38 & - & -. & -. & -- & 259 & 1 & -1.54 & - & 7.6 & -. & -. & -- \\
\hline 7 & 4 & -0.04 & -. & -. & 8.6 & -- & -. & 265 & 3 & 0.71 & -- & - & 9.1 & -- & - \\
\hline 8 & 0 & -2.59 & -. & -- & - & - & 6.9 & 305 & 3 & 0.56 & -- & 9 & -- & -- & - \\
\hline 10 & 3 & 1.01 & - & - & - & .. & 9.3 & 328 & 3 & -0.79 & - & - & 8.1 & . & - \\
\hline 12 & 3 & -0.94 & 8 & 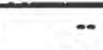 & $\cdots$ & - & $-\cdot$ & 330 & 3 & 1.01 & - & -- & 9.3 & 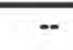 & - \\
\hline 16 & 0 & 2.06 & -. & 10 & - & -- & -. & 356 & 4 & -0.12 & -. & - & 8.55 & -. & - \\
\hline 23 & 0 & 2.52 & -. & 10.31 & - & -- & -. & 370 & 0 & -3.73 & 6.14 & -- & -. & - & -- \\
\hline 25 & 3 & -0.79 & 8.1 & - & - & -- & - & 372 & 3 & -0.94 & - & 8 & - & -. & -- \\
\hline 26 & 4 & -0.49 & -. & .- & $=$ & - & 8.3 & & & & & & & & \\
\hline
\end{tabular}


Table 11. Statistical summary of reported data for standard reference sample T-169 (trace constituents) -- continued

T-169 BORON (B) in $\mu g / L$

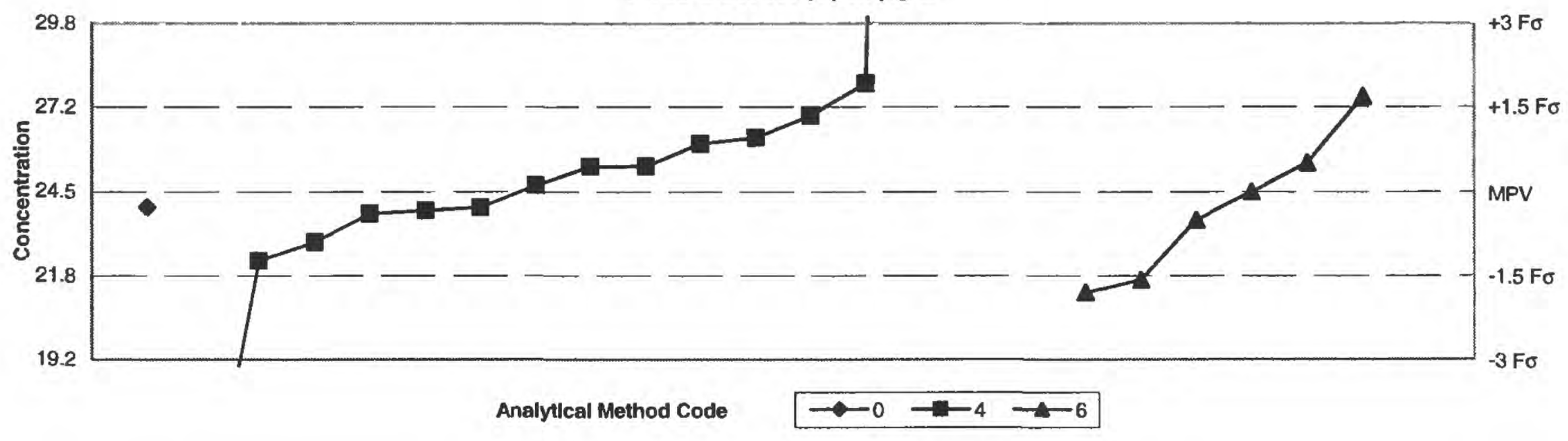

\begin{tabular}{|c|c|c|c|c|c|c|}
\hline \multirow[t]{2}{*}{ SUMMARY } & \multicolumn{4}{|c|}{ Methods } & \multicolumn{2}{|c|}{ Statlstics } \\
\hline & 0 & 4 & 6 & Method Codes & & \\
\hline$n=$ & 1 & 14 & 6 & 00 Other & MPV $=$ & $24.5 \mu \mathrm{g} / \mathrm{L}$ \\
\hline Minimum = & 24 & 13 & 21.3 & 04 Inductively coupled plasma & F-pseudosigma $=$ & 1.8 \\
\hline Maximum $=$ & & 80 & 27.5 & 06 Inductively coupled plasma/mass spectrometry & $n=$ & 21 \\
\hline Median $=$ & & 25.0 & 24.1 & & $\mathrm{Uh}=$ & 26.0 \\
\hline F-pseudosigma $=$ & & 1.8 & 2.8 & & Lh $=$ & 23.6 \\
\hline
\end{tabular}

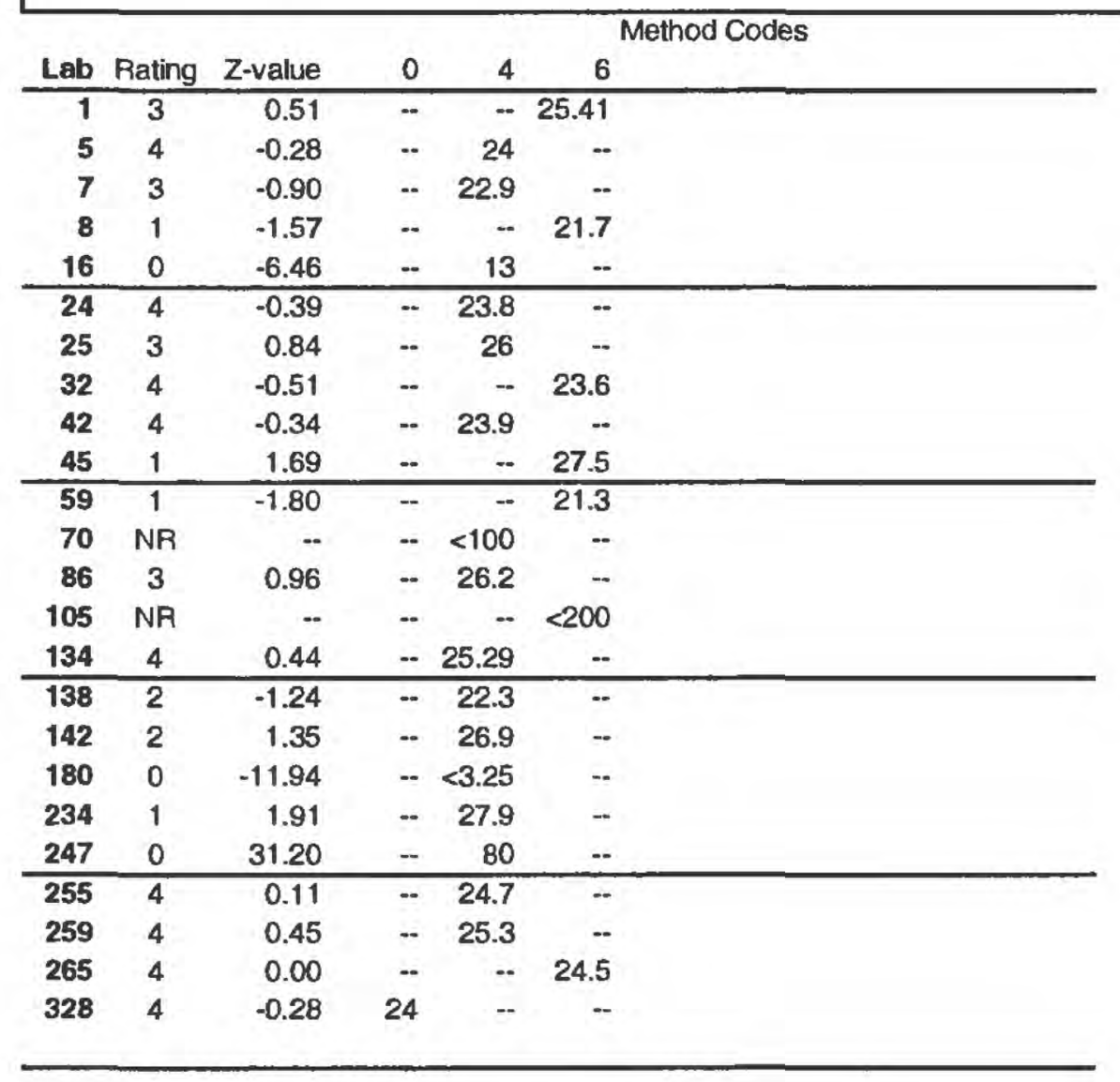


Table 11. Statistical summary of reported data for standard reference sample $T$-169 (trace constituents) -- continued

T-169 BARIUM (Ba) in $\mu g / L$

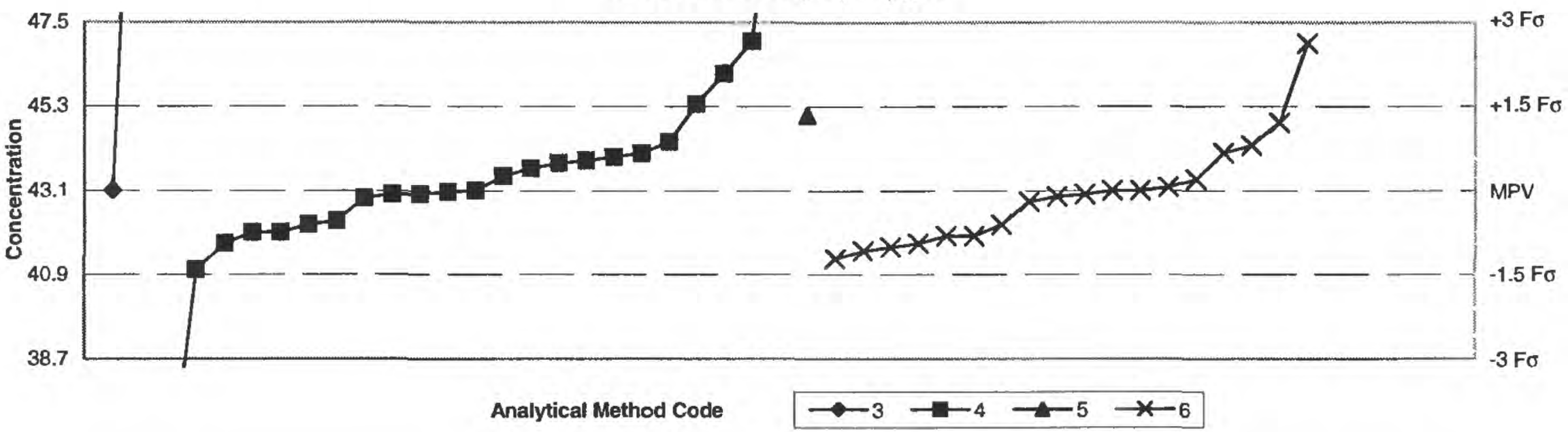

\begin{tabular}{|c|c|c|c|c|c|c|}
\hline \multirow[t]{2}{*}{ SUMMARY } & \multicolumn{4}{|c|}{ Methods } & \multicolumn{2}{|c|}{ Statistics } \\
\hline & 3 & 4 & 5 & Method Codes & & \\
\hline$n=$ & $\overline{2}$ & 23 & 18 & 03 Atomic absorption: graphite fumace & MPV $=$ & $43.1 \mu \mathrm{g} / \mathrm{L}$ \\
\hline Minimum = & 43.1 & 35 & 45.141 .29 & 04 Inductively coupled plasma & F-pseudosigma $=$ & 1.5 \\
\hline Maximum $=$ & 58.9 & 52.7 & 47 & 05 Direct current plasma & Rating criterion $=$ & 2.2 \\
\hline Median $=$ & & 43.1 & 43.0 & 06 Inductively coupled plasma/mass spectrometry & $\mathrm{n}=$ & 44 \\
\hline F-pseudosigma $=$ & & 1.3 & 1.1 & & $\begin{array}{l}\mathrm{Uh}= \\
\mathrm{Lh}=\end{array}$ & $\begin{array}{l}44.1 \\
42.1\end{array}$ \\
\hline
\end{tabular}

\begin{tabular}{|c|c|c|c|c|c|c|}
\hline \multirow[b]{2}{*}{ Lab } & \multirow[b]{2}{*}{ Rating } & \multirow[b]{2}{*}{ Z-value } & \multicolumn{4}{|c|}{ Method Codes } \\
\hline & & & 3 & 4 & 5 & 6 \\
\hline 1 & 4 & -0.07 & - & - & $\cdots$ & 42.94 \\
\hline 5 & 4 & 0.46 & - & 44.1 & - & -- \\
\hline 7 & 4 & -0.42 & - & 42.2 & - & -- \\
\hline 8 & 3 & 0.56 & - & -- & - & 44.3 \\
\hline 16 & 3 & -0.97 & - & 41 & $\because$ & - \\
\hline 23 & 4 & -0.01 & 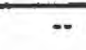 & 43.07 & - & $\cdots$ \\
\hline 24 & 2 & 1.07 & - & 45.4 & - & - \\
\hline 25 & 0 & -3.76 & - & 35 & - & -. \\
\hline 32 & 4 & -0.14 & - & -. & - & 42.8 \\
\hline 33 & 3 & 0.93 & - & $\because$ & 45.1 & -- \\
\hline 42 & 4 & 0.05 & - & 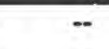 & - & 43.2 \\
\hline 45 & 3 & -0.56 & - & - & - & 41.9 \\
\hline 46 & 4 & 0.00 & - & 43.1 & - & - \\
\hline 59 & 4 & 0.00 & - & -. & - & 43.1 \\
\hline 70 & 4 & 0.00 & - & -- & - & 43.1 \\
\hline 86 & 3 & -0.65 & - & 41.7 & $=$ & - \\
\hline 89 & 0 & 7.33 & 58.9 & -- & - & -. \\
\hline 97 & 4 & -0.37 & - & 42.3 & - & .. \\
\hline 105 & 4 & -0.05 & - & - & - & 43 \\
\hline 113 & 4 & -0.09 & $\because$ & 42.9 & $=$ & - \\
\hline 121 & 1 & 1.81 & - & - & $=$ & 47 \\
\hline 134 & 4 & 0.34 & - & 43.84 & - & -. \\
\hline 138 & 4 & 0.19 & - & 43.5 & - & -. \\
\hline 142 & 3 & 0.84 & - & -. & $\cdots$ & 44.9 \\
\hline 146 & 4 & 0.42 & - & 44 & $=$ & -. \\
\hline 149 & 4 & 0.46 & - & -. & - & 44.1 \\
\hline 180 & 3 & -0.74 & -- & -- & - & 41.5 \\
\hline 183 & 4 & 0.00 & 43.1 & - & - & -- \\
\hline 198 & 3 & -0.65 & -- & -. & - & 41.7 \\
\hline 212 & 2 & 1.44 & - & 46.2 & $\because$ & -- \\
\hline 220 & 1 & 1.84 & -- & 47.06 & $\cdots$ & - \\
\hline 234 & 4 & 0.37 & - & 43.9 & $\cdots$ & -. \\
\hline 245 & 3 & -0.84 & -- & -- & - & 41.29 \\
\hline 247 & 3 & -0.56 & -- & -- & - & 41.9 \\
\hline 256 & 3 & 0.60 & - & 44.4 & $=$ & -- \\
\hline 259 & 4 & 0.28 & -- & 43.7 & - & -- \\
\hline 265 & 4 & -0.05 & -. & 43 & $\cdots$ & -. \\
\hline 304 & 4 & 0.14 & - & - & $\cdots$ & 43.4 \\
\hline 305 & 3 & -0.51 & -. & 42 & $\cdots$ & -- \\
\hline 328 & 4 & -0.05 & .. & 43 & $\because$ & -. \\
\hline
\end{tabular}

\begin{tabular}{|c|c|c|c|c|c|c|}
\hline \multirow[b]{2}{*}{ Lab } & \multirow[b]{2}{*}{ Rating } & \multirow[b]{2}{*}{ Z-value } & \multicolumn{4}{|c|}{ Method Codes } \\
\hline & & & 3 & 4 & 5 & 6 \\
\hline 330 & 4 & -0.42 & 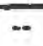 & -- & - & 42.2 \\
\hline 356 & 3 & -0.70 & -- & -- & -. & 41.6 \\
\hline 370 & 0 & 4.45 & -. & 52.7 & -. & -. \\
\hline 372 & 3 & -0.51 & - & 42 & - & -. \\
\hline
\end{tabular}


Table 11. Statistical summary of reported data for standard reference sample T-169 (trace constituents) -- continued

T-169 BERYLLIUM (Be) in $\mu g / L$

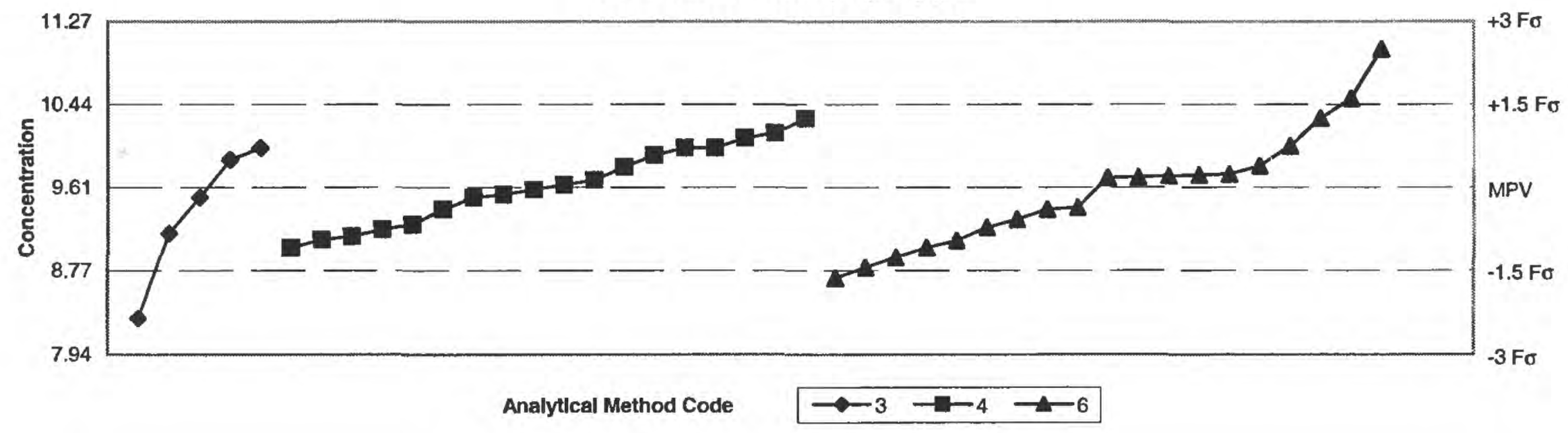

\begin{tabular}{|c|c|c|c|c|c|c|}
\hline \multirow[t]{2}{*}{ SUMMARY } & \multicolumn{4}{|c|}{ Methods } & \multicolumn{2}{|c|}{ Stat|stics } \\
\hline & 3 & 4 & 6 & Method Codes & & \\
\hline$n=$ & 5 & 18 & 19 & 03 Atomic absorption: graphite fumace & MPV $=$ & $9.61 \mu \mathrm{g} / \mathrm{L}$ \\
\hline Minimum $=$ & 8.3 & 9 & 8.7 & 04 Inductively coupled plasma & F-pseudosigma $=$ & 0.56 \\
\hline Maximum $=$ & 10 & 10.3 & 11 & 06 Inductively coupled plasma/mass spectrometry & $n=$ & 42 \\
\hline Median = & 9.50 & 9.61 & 9.70 & & $U_{h}=$ & 9.93 \\
\hline F-pseudosigma $=$ & 0.55 & 0.57 & 0.48 & & $\operatorname{Lh}=$ & 9.18 \\
\hline
\end{tabular}

\begin{tabular}{|c|c|c|c|c|c|c|c|c|c|c|c|}
\hline \multicolumn{9}{|c|}{ Method Codes } & \multirow{2}{*}{\multicolumn{3}{|c|}{$6^{\text {Method Codes }}$}} \\
\hline Lab & Rating & $Z$-value & 3 & 4 & 6 & Lab & Rating & $Z$-value & & & \\
\hline 1 & 4 & -0.13 & -- & 9.53 & - & 370 & 3 & 0.71 & 10 & $\overline{-}$ & - \\
\hline 5 & 4 & -0.40 & -- & 9.38 & .. & 372 & 2 & -1.09 & -- & 9 & .. \\
\hline 7 & 2 & -1.45 & -. & -- & 8.8 & & & & & & \\
\hline 8 & 2 & -1.27 & -- & -. & 8.9 & & & & & & \\
\hline 16 & 3 & 0.71 & - & 10 & - & & & & & & \\
\hline 23 & 4 & 0.37 & $\ldots$ & 9.81 & - & & & & & & \\
\hline 25 & 3 & 0.71 & -. & 10 & -. & & & & & & \\
\hline 26 & 3 & -0.76 & -- & 9.18 & -- & & & & & & \\
\hline 32 & 1 & -1.63 & -- & .. & 8.7 & & & & & & \\
\hline 42 & 4 & 0.22 & -- & -- & 9.73 & & & & & & \\
\hline 45 & 3 & -0.58 & $\ldots$ & - & 9.28 & & & & & & \\
\hline 46 & 4 & 0.13 & -. & 9.68 & -- & & & & & & \\
\hline 59 & 4 & 0.24 & -. & -- & 9.74 & & & & & & \\
\hline 70 & 2 & 1.25 & -. & -. & 10.3 & & & & & & \\
\hline 86 & 3 & -0.94 & -. & 9.08 & - & & & & & & \\
\hline 89 & 0 & -2.35 & 8.3 & - & - & & & & & & \\
\hline 97 & 4 & 0.49 & 9.88 & -- & $-\cdot$ & & & & & & \\
\hline 105 & 2 & -1.09 & -- & .. & 9 & & & & & & \\
\hline 113 & 3 & 0.89 & -- & 10.1 & -. & & & & & & \\
\hline 121 & 4 & -0.37 & -- & .. & 9.4 & & & & & & \\
\hline 134 & 3 & -0.67 & $\overline{--}$ & 9.23 & - & & & & & & \\
\hline 138 & 4 & -0.04 & .. & 9.58 & .- & & & & & & \\
\hline 142 & 4 & 0.39 & -. & -- & 9.82 & & & & & & \\
\hline 146 & 3 & -0.89 & -- & 9.11 & .- & & & & & & \\
\hline 149 & 4 & 0.17 & .. & -- & 9.7 & & & & & & \\
\hline 180 & 3 & -0.96 & .. & - & 9.07 & & & & & & \\
\hline 183 & 3 & -0.84 & 9.14 & -- & -. & & & & & & \\
\hline 193 & 4 & -0.19 & 9.5 & - & -. & & & & & & \\
\hline 198 & 4 & 0.21 & -- & -- & 9.72 & & & & & & \\
\hline 212 & 4 & -0.19 & -. & 9.5 & -. & & & & & & \\
\hline 220 & 4 & 0.04 & - & 9.63 & -. & & & & & & \\
\hline 234 & 3 & 0.58 & -. & 9.93 & -- & & & & & & \\
\hline 245 & 3 & 0.75 & .- & -. & 10.02 & & & & & & \\
\hline 247 & 4 & 0.19 & -. & .- & 9.71 & & & & & & \\
\hline 256 & 3 & 0.98 & -- & 10.15 & - & & & & & & \\
\hline 265 & 1 & 1.61 & -. & - & 10.5 & & & & & & \\
\hline 305 & 2 & 1.25 & -- & 10.3 & .. & & & & & & \\
\hline 328 & 3 & -0.73 & -. & -. & 9.2 & & & & & & \\
\hline 330 & 0 & 2.51 & -- & -. & 11 & & & & & & \\
\hline 356 & 4 & -0.40 & .- & - & 9.38 & & & & & & \\
\hline
\end{tabular}


Table 11. Statistical summary of reported data for standard reference sample T-169 (trace constituents) - continued

T-169 CALCIUM (Ca) in $\mathrm{mg} / \mathrm{L}$

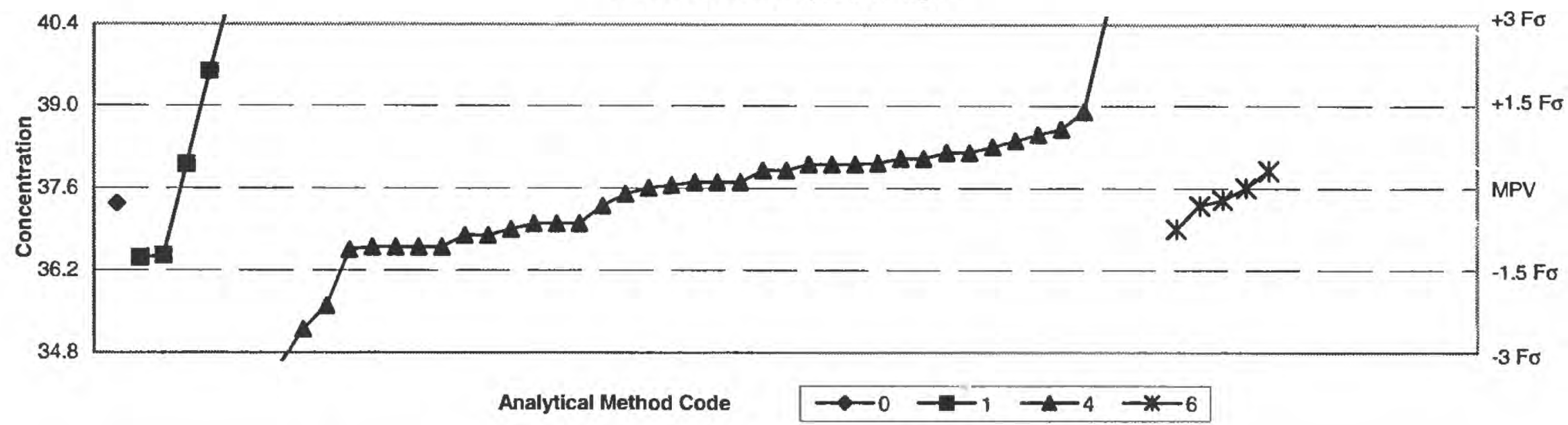

\begin{tabular}{|c|c|c|c|c|c|c|c|c|}
\hline \multirow[t]{2}{*}{ SUMMARY } & \multicolumn{6}{|c|}{ Methods } & \multicolumn{2}{|c|}{ Statistles } \\
\hline & 0 & 1 & 4 & 5 & 6 & Method Codes & & \\
\hline$n=$ & 1 & 5 & 39 & 1 & 5 & 00 Other & MPV = & $37.6 \mathrm{mg} / \mathrm{L}$ \\
\hline Minimum $=$ & 37.34 & 36.42 & 34.3 & 33.2 & 36.9 & 01 Atomic absorption: direct, air & F-pseudosigma $=$ & 0.9 \\
\hline Maximum $=$ & & 41.1 & 40.9 & & 37.89 & 04 inductively coupled plasma & Rating criterion = & 1.9 \\
\hline Median $=$ & & 38.0 & 37.7 & & 37.4 & 05 Direct current plasma & $n=$ & 51 \\
\hline F-pseudosigma $=$ & & 2.3 & 1.0 & & 0.2 & 06 inductively coupled plasma/m & $\mathrm{Uh}=$ & 38.1 \\
\hline
\end{tabular}

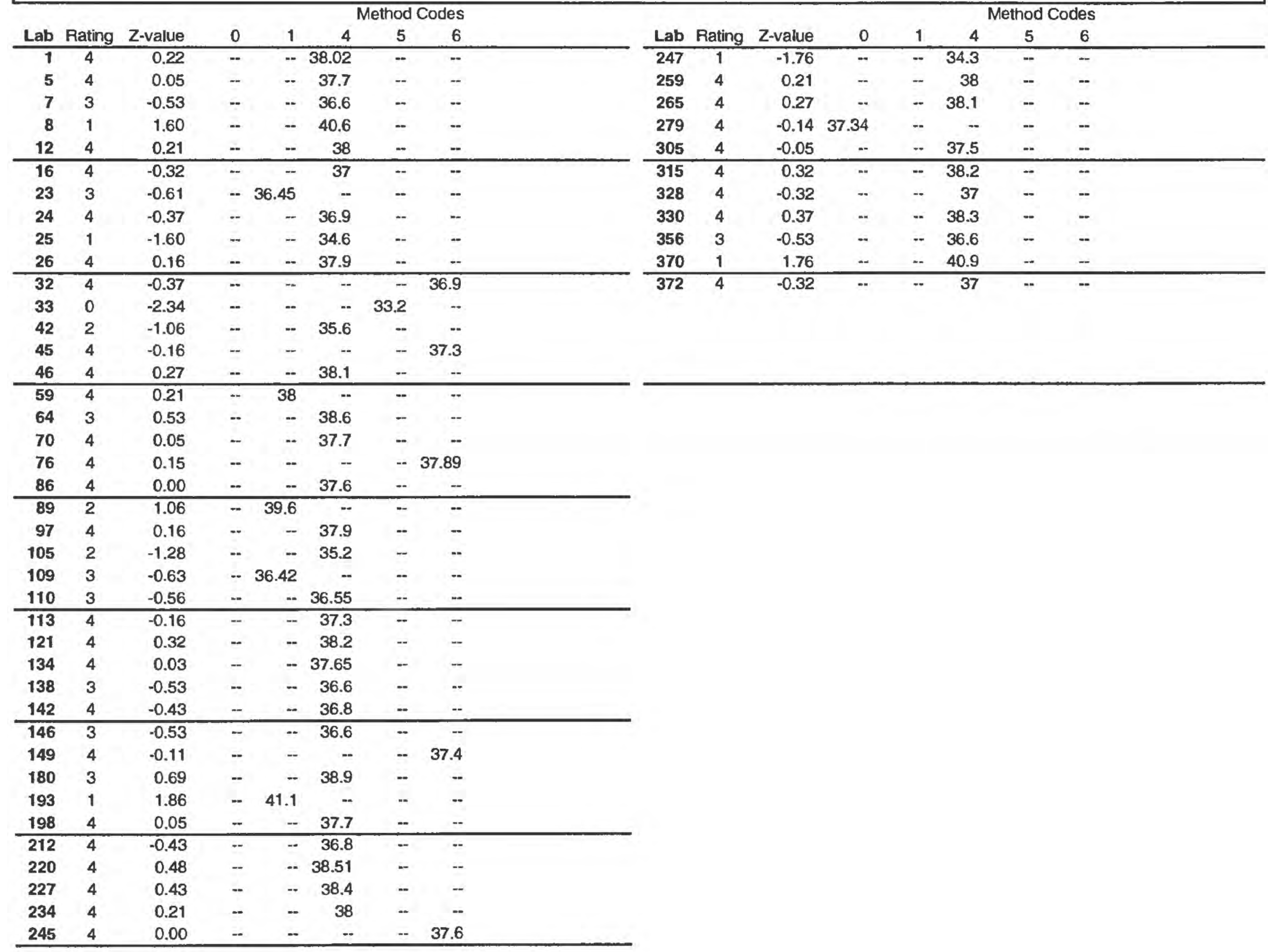


Table 11. Statistical summary of reported data for standard reference sample T-169 (trace constituents) -- continued

T-169 CADMIUM (Cd) in $\mu \mathrm{g} / \mathrm{L}$

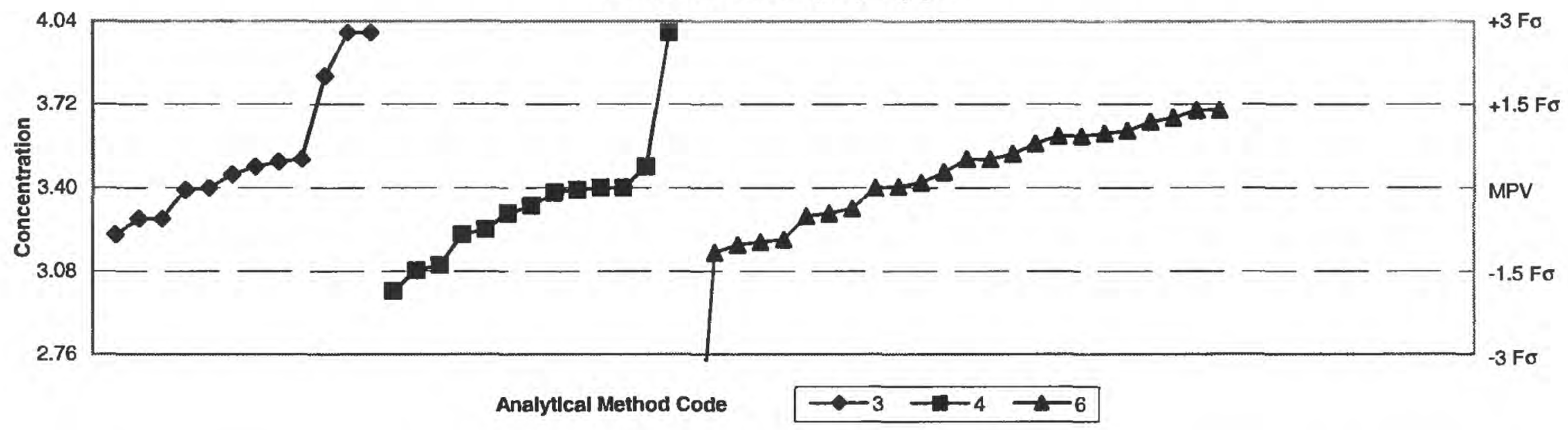

\begin{tabular}{|c|c|c|c|c|c|c|}
\hline \multirow[t]{2}{*}{ SUMMARY } & \multicolumn{4}{|c|}{ Methods } & \multicolumn{2}{|c|}{ Statistics } \\
\hline & 3 & 4 & 6 & Method Codes & & \\
\hline$n=$ & 12 & 13 & 24 & 03 Atomic absorption: graphite fumace & MPV $=$ & $3.40 \mu \mathrm{g} / \mathrm{L}$ \\
\hline Minimum $=$ & 3.22 & 3 & 2 & 04 Inductively coupled plasma & F-pseudosigma $=$ & 0.21 \\
\hline Maximum $=$ & 4 & 4 & 3.7 & 06 Inductively coupled plasma/mass spectrometry & $n=$ & 49 \\
\hline Median $=$ & 3.47 & 3.33 & 3.49 & & $\mathrm{Uh}=$ & 3.57 \\
\hline F-pseudosigma $=$ & 0.25 & 0.13 & 0.23 & & $\operatorname{Ln}=$ & 3.28 \\
\hline
\end{tabular}

\begin{tabular}{|c|c|c|c|c|c|c|c|c|c|c|c|}
\hline \multicolumn{9}{|c|}{ Method Codes } & \multirow{2}{*}{\multicolumn{3}{|c|}{${ }_{6}$ Method Codes }} \\
\hline Lab & Rating & Z-value & 3 & 4 & 6 & Lab & Rating & Z-value & & & \\
\hline 1 & 3 & 0.98 & -- & - & 3.61 & 256 & 4 & 0.37 & - & 3.48 & - \\
\hline 5 & 3 & -0.84 & 3.22 & -. & - & 259 & 4 & 0.00 & - & 3.4 & - \\
\hline 7 & 3 & -0.93 & -- & - & 3.2 & 265 & 3 & 0.93 & - & - & 3.6 \\
\hline 8 & 2 & 1.40 & - & - & 3.7 & 304 & 4 & 0.28 & - & -- & 3.46 \\
\hline 10 & 4 & 0.47 & 3.5 & - & -- & 305 & 4 & 0.00 & - & 3.4 & - \\
\hline 12 & 0 & 2.79 & 4 & - & -- & 328 & 4 & -0.47 & - & $-\cdots$ & 3.3 \\
\hline 16 & 0 & 2.79 & - & 4 & - & 330 & 2 & 1.40 & - & - & 3.7 \\
\hline 23 & 4 & -0.09 & -. & 3.38 & -. & 356 & 3 & 0.60 & $\ldots$ & -. & 3.53 \\
\hline 25 & 4 & 0.00 & 3.4 & - & -. & 370 & 4 & 0.37 & 3.48 & - & -- \\
\hline 26 & 4 & 0.23 & 3.45 & .- & - & 372 & 1 & -1.86 & - & 3 & - \\
\hline 32 & 3 & 0.93 & $=$ & $\overline{-}$ & 3.6 & & & & & & \\
\hline 42 & 2 & -1.02 & - & - & 3.18 & & & & & & \\
\hline 45 & 2 & -1.16 & - & - & 3.15 & & & & & & \\
\hline 46 & 3 & -0.56 & 3.28 & - & - & & & & & & \\
\hline 59 & NR & -- & - & -. & $<5$ & & & & & & \\
\hline 70 & 3 & 0.51 & - & -- & 3.51 & & & & & & \\
\hline 76 & 4 & 0.02 & -- & -. & 3.404 & & & & & & \\
\hline 86 & 2 & -1.49 & - & 3.08 & -- & & & & & & \\
\hline 89 & 0 & 2.79 & 4 & .. & -- & & & & & & \\
\hline 97 & 3 & 0.51 & 3.51 & -. & - & & & & & & \\
\hline 105 & 4 & -0.37 & - & -. & 3.32 & & & & & & \\
\hline 113 & 3 & -0.74 & - & 3.24 & -- & & & & & & \\
\hline 121 & 0 & -6.51 & - & -- & 2 & & & & & & \\
\hline 134 & 4 & -0.33 & - & 3.33 & -- & & & & & & \\
\hline 138 & 3 & 0.79 & - & -. & 3.57 & & & & & & \\
\hline 142 & 2 & 1.26 & - & - & 3.67 & & & & & & \\
\hline 146 & 3 & -0.84 & - & 3.22 & - & & & & & & \\
\hline 147 & 3 & -0.98 & - & -- & 3.19 & & & & & & \\
\hline 149 & 4 & 0.00 & - & -- & 3.4 & & & & & & \\
\hline 180 & 3 & -0.51 & - & - & 3.29 & & & & & & \\
\hline 183 & 0 & 2.00 & 3.83 & - & - & & & & & & \\
\hline 190 & 3 & -0.56 & 3.28 & - & -. & & & & & & \\
\hline 193 & 4 & -0.05 & 3.39 & - & -. & & & & & & \\
\hline 198 & 2 & 1.02 & -- & -- & 3.62 & & & & & & \\
\hline 212 & 2 & -1.40 & $\cdots$ & 3.1 & - & & & & & & \\
\hline 227 & 4 & -0.05 & - & 3.39 & - & & & & & & \\
\hline 234 & 4 & -0.47 & -- & 3.3 & - & & & & & & \\
\hline 245 & 2 & 1.18 & -- & -- & 3.654 & & & & & & \\
\hline 247 & 4 & 0.09 & .. & .- & 3.42 & & & & & & \\
\hline 255 & 3 & 0.51 & - & - & 3.51 & & & & & & \\
\hline
\end{tabular}


Table 11. Statistical summary of reported data for standard reference sample T-169 (trace constituents) -- continued

T-169 COBALT (Co) in $\mu \mathrm{g} / \mathrm{L}$

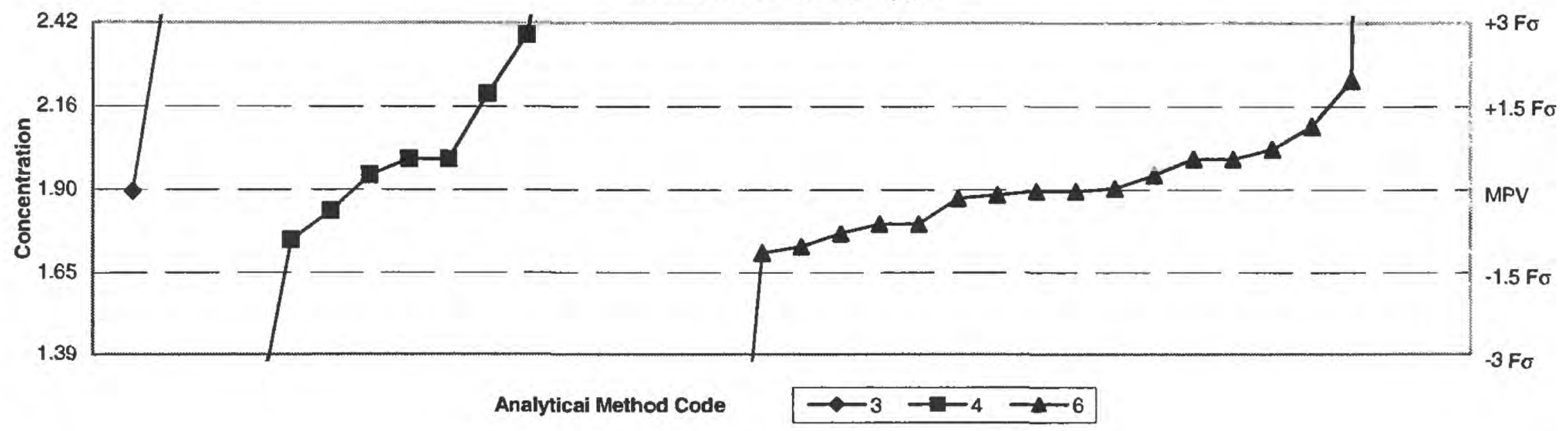

\begin{tabular}{|c|c|c|c|c|c|c|}
\hline \multirow[t]{2}{*}{ SUMMARY } & \multicolumn{4}{|c|}{ Methods } & \multicolumn{2}{|c|}{ Statistics } \\
\hline & 3 & 4 & 6 & Method Codes & & \\
\hline$n=$ & 2 & 10 & 18 & 03 Atomic absorption: graphite fumace & MPV $=$ & $1.91 \mu \mathrm{g} / \mathrm{L}$ \\
\hline Minimum = & 1.9 & 1.1 & 0.35 & 04 Inductively coupled plasma & F-pseudosigma $=$ & 0.17 \\
\hline Maximum $=$ & 2.65 & 3 & 10.2 & 06 Inductively coupled plasma/mass spectrometry & $n=$ & 30 \\
\hline Median $=$ & & 1.98 & 1.90 & & $\mathrm{Uh}=$ & 2.03 \\
\hline F-pseudosigma = & & 0.33 & 0.15 & & $\operatorname{Lh}=$ & 1.80 \\
\hline
\end{tabular}

\begin{tabular}{|c|c|c|c|c|c|}
\hline \multirow[b]{2}{*}{ Lab } & \multirow[b]{2}{*}{ Rating } & \multirow[b]{2}{*}{ Z-vaiue } & \multicolumn{3}{|r|}{ Method Codes } \\
\hline & & & 3 & 4 & 6 \\
\hline 1 & 3 & 0.73 & 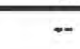 & -- & 2.03 \\
\hline 5 & NR & -. & -. & $<3.00$ & -. \\
\hline 7 & 0 & 48.65 & - & -- & 10.2 \\
\hline 8 & 3 & 0.56 & - & -- & 2 \\
\hline 16 & 1 & 1.73 & 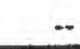 & 2.2 & $\ldots$ \\
\hline 25 & NR & -. & - & $<4$ & $\overline{-}$ \\
\hline 32 & 3 & 0.56 & - & .. & 2 \\
\hline 42 & NR & .- & .. & -- & $<2$ \\
\hline 45 & 4 & 0.03 & -. & $\cdots$ & 1.91 \\
\hline 59 & NR & - & - & - & $<5$ \\
\hline 70 & $\overline{N R}$ & $\because$ & $\ldots$ & $\bar{x}$ & $<10$ \\
\hline 89 & 4 & -0.03 & 1.9 & - & - \\
\hline 97 & 0 & 4.37 & 2.65 & -. & -- \\
\hline 105 & NR & -- & - & .. & $<50$ \\
\hline 121 & 0 & -9.12 & .. & - & 0.35 \\
\hline 134 & $\overline{4}$ & -0.38 & $\cdots$ & 1.84 & -- \\
\hline 138 & 4 & 0.26 & .. & -- & 1.95 \\
\hline 142 & 4 & -0.15 & -- & - & 1.88 \\
\hline 146 & 0 & 2.79 & - & 2.38 & - \\
\hline 147 & 2 & -1.14 & - & .. & 1.71 \\
\hline 149 & 4 & -0.03 & -. & 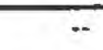 & 1.9 \\
\hline 180 & 2 & -1.03 & - & -. & 1.73 \\
\hline 198 & 3 & -0.62 & -. & -- & 1.8 \\
\hline 212 & 0 & -4.72 & - & 1.1 & - \\
\hline 234 & 4 & 0.26 & - & 1.95 & -. \\
\hline 245 & 1 & 1.96 & - & $\cdots$ & 2.24 \\
\hline 247 & 3 & -0.79 & -- & -- & 1.77 \\
\hline 256 & 3 & 0.56 & - & 2 & - \\
\hline 259 & 3 & -0.91 & - & 1.75 & + \\
\hline 265 & 3 & -0.62 & - & -- & 1.8 \\
\hline 304 & 4 & -0.09 & .. & $\ldots$ & 1.89 \\
\hline 305 & 3 & 0.56 & -- & 2 & -. \\
\hline 328 & 0 & -4.72 & - & 1.1 & -. \\
\hline 330 & 2 & 1.14 & - & -. & 2.1 \\
\hline 356 & 4 & -0.03 & - & -. & 1.9 \\
\hline 370 & $\overline{\mathrm{NR}}$ & - & - & $<500$ & - \\
\hline 372 & 0 & 6.42 & -- & 3 & - \\
\hline
\end{tabular}


Table 11. Statistical summary of reported data for standard reference sample T-169 (trace constituents) -- continued

T-169 CHROMIUM $(\mathrm{Cr})$ in $\mu \mathrm{g} / \mathrm{L}$

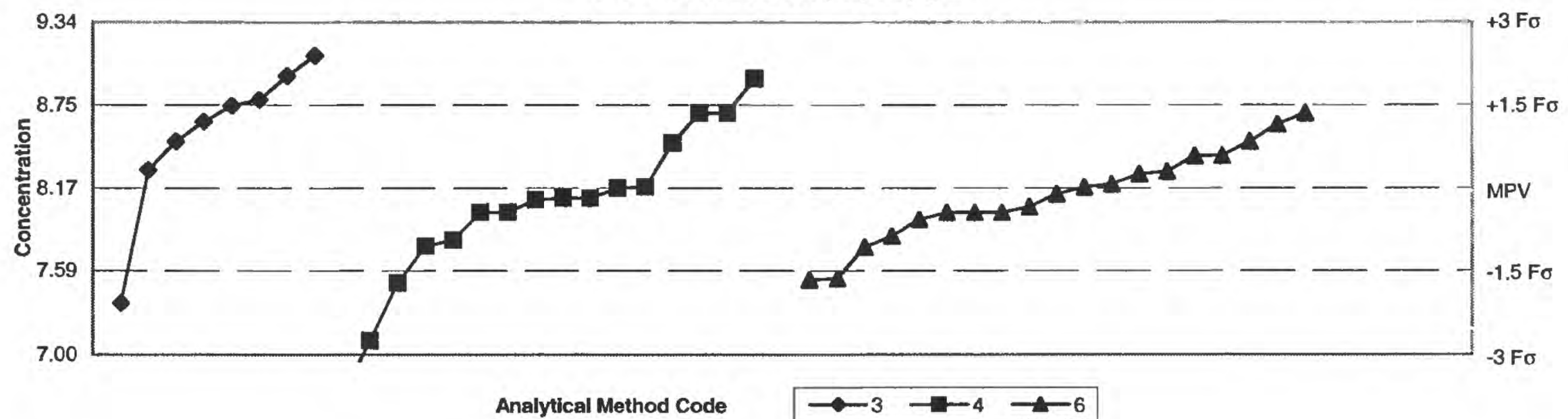

\begin{tabular}{|c|c|c|c|c|c|c|}
\hline \multirow[t]{2}{*}{ SUMMARY } & \multicolumn{4}{|c|}{ Methods } & \multicolumn{2}{|c|}{ Statlstles } \\
\hline & 3 & 4 & 6 & Method Codes & & \\
\hline $\mathbf{n}=$ & 8 & 16 & 19 & 03 Atomic absorption: graphite furnace & MPV $=$ & $8.17 \mu \mathrm{g} / \mathrm{L}$ \\
\hline Minimurn $=$ & 7.36 & 6.7 & 7.52 & 04 Inductively coupled plasma & F-pseudosigrna $=$ & 0.39 \\
\hline Maximurn $=$ & 9.1 & 8.94 & 8.7 & 06 Inductively coupled plasma/rnass spectrornetry & Rating criterion $=$ & 0.41 \\
\hline Median $=$ & 8.70 & 8.10 & 8.13 & & $n=$ & 43 \\
\hline F-pseudosigma $=$ & 0.35 & 0.41 & 0.27 & & $\mathrm{Uh}=$ & 8.50 \\
\hline & & & & & $\operatorname{Lh}=$ & 7.98 \\
\hline
\end{tabular}

\begin{tabular}{|c|c|c|c|c|c|c|c|c|c|c|c|}
\hline \multicolumn{9}{|c|}{ Method Codes } & \multicolumn{3}{|r|}{ Method Codes } \\
\hline Lab & Rating & Z-value & 3 & 4 & 6 & Lab & Rating & Z-value & 3 & 4 & 6 \\
\hline 1 & 2 & 1.42 & 8.75 & - & 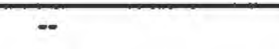 & 328 & 3 & -0.91 & - & 7.8 & -- \\
\hline 5 & 1 & 1.88 & - & 8.94 & - & 330 & 2 & 1.30 & -- & -- & 8.7 \\
\hline 7 & 3 & 0.56 & -. & - & 8.4 & 356 & 4 & 0.02 & - & - & 8.18 \\
\hline 8 & 4 & 0.07 & -. & - & 8.2 & 370 & 4 & 0.02 & -- & 8.18 & -- \\
\hline 10 & 0 & 2.28 & 9.1 & -- & - & 372 & 2 & 1.30 & -. & 8.7 & $\ldots$ \\
\hline
\end{tabular}


Table 11. Statistical summary of reporfed data for standard reference sample T-169 (trace constituents) -- continued

$T-169$ COPPER $(\mathrm{Cu})$ in $\mu \mathrm{g} / \mathrm{L}$

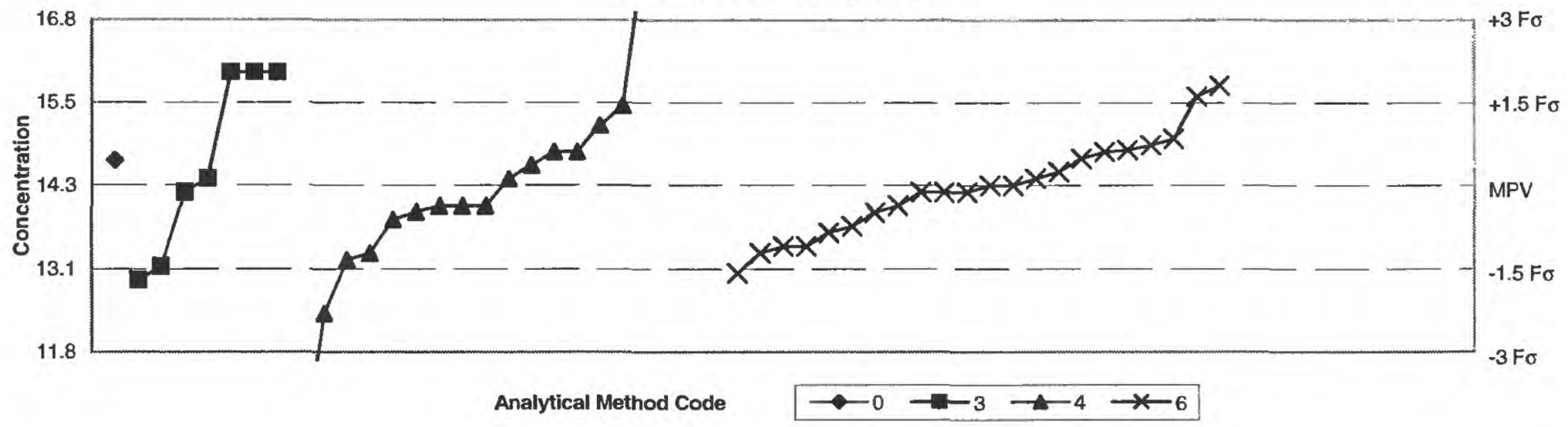

\begin{tabular}{|c|c|c|c|c|c|c|c|}
\hline \multirow[t]{2}{*}{ SUMMARY } & \multicolumn{5}{|c|}{ Methods } & \multicolumn{2}{|c|}{ Statlstics } \\
\hline & 0 & 3 & 4 & 6 & Method Codes & & \\
\hline$n=$ & 1 & 7 & 18 & 22 & 00 Other & MPV $=$ & $14.3 \mu \mathrm{g} / \mathrm{L}$ \\
\hline Minimum $=$ & 14.67 & 12.9 & 10 & 13 & 03 Atomic absorption: graphite fumace & F-pseudosigma $=$ & 0.8 \\
\hline Maximum $=$ & & 16 & 23 & 15.8 & 04 Inductively coupled plasma & $n=$ & 48 \\
\hline Median $=$ & & 14.4 & 14.2 & 14.3 & 06 Inductively coupled plasma/mass spe & ometry & 14.9 \\
\hline F-pseudosigma $=$ & & 1.7 & 1.0 & 0.8 & & $\mathrm{Lh}=$ & 13.8 \\
\hline
\end{tabular}

\begin{tabular}{|c|c|c|c|c|c|c|c|c|c|c|c|c|c|}
\hline \multicolumn{10}{|c|}{ Method Codes } & \multicolumn{4}{|r|}{ Method Codes } \\
\hline Lab & Rating & Z-value & 0 & 3 & 4 & 6 & Lab & Rating & Z-value & 0 & 3 & 4 & 6 \\
\hline 1 & 3 & 0.64 & - & $\overline{-}$ & - & 14.83 & 259 & 4 & 0.12 & - & - & 14.4 & $\cdots$ \\
\hline 5 & 3 & 0.60 & - & - & 14.8 & - & 265 & 4 & 0.12 & - & -- & - & 14.4 \\
\hline 7 & 3 & 0.60 & - & - & 14.8 & -- & 304 & 4 & 0.00 & - & - & - & 14.3 \\
\hline 8 & 1 & 1.81 & - & - & -. & 15.8 & 305 & 4 & -0.36 & -- & $\cdots$ & 14 & - \\
\hline 10 & 1 & -1.69 & - & 12.9 & 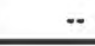 & -- & 328 & 0 & 10.53 & $\cdots$ & $\cdots$ & 23 & $\cdots$ \\
\hline 12 & 0 & 2.06 & - & 16 & - & - & 330 & 3 & 0.60 & - & - & 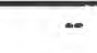 & 14.8 \\
\hline 16 & 0 & -5.20 & $\cdots$ & - & 10 & - & 356 & 4 & 0.48 & -. & - & -- & 14.7 \\
\hline 23 & 4 & 0.45 & 14.67 & - & - & - & 370 & NR & - & - & - & $<20.0$ & - \\
\hline 24 & 0 & 4.60 & - & .. & 18.1 & - & 372 & 4 & -0.36 & -- & - & 14 & -. \\
\hline 25 & 4 & -0.36 & - & $=$ & 14 & -. & & & & & & & \\
\hline 26 & 3 & -0.60 & - & - & 13.8 & - & & & & & & & \\
\hline 32 & 4 & -0.12 & - & - & -- & 14.2 & & & & & & & \\
\hline 42 & 2 & -1.09 & - & - & - & 13.4 & & & & & & & \\
\hline 45 & 4 & 0.00 & -. & -- & - & 14.3 & & & & & & & \\
\hline 46 & 2 & -1.45 & - & 13.1 & - & - & & & & & & & \\
\hline 59 & 4 & -0.12 & - & $\cdots$ & - & 14.2 & & & & & & & \\
\hline 70 & 3 & -0.73 & - & - & - & 13.7 & & & & & & & \\
\hline 86 & 2 & 1.45 & -- & - & 15.5 & -- & & & & & & & \\
\hline 89 & 4 & 0.12 & -- & 14.4 & -. & - & & & & & & & \\
\hline 97 & 0 & 2.06 & 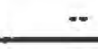 & 16 & $=$ & - & & & & & & & \\
\hline 105 & 3 & 0.85 & -- & 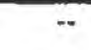 & - & 15 & & & & & & & \\
\hline 113 & 2 & 1.09 & - & - & 15.2 & - & & & & & & & \\
\hline 121 & 4 & -0.36 & - & - & -- & 14 & & & & & & & \\
\hline 134 & 4 & -0.47 & -- & - & 13.91 & - & & & & & & & \\
\hline 138 & 3 & -0.85 & $=$ & - & -- & 13.6 & & & & & & & \\
\hline 142 & 2 & -1.09 & - & - & - & 13.4 & & & & & & & \\
\hline 146 & 2 & -1.21 & - & - & 13.3 & - & & & & & & & \\
\hline 147 & 4 & -0.12 & -. & - & - & 14.2 & & & & & & & \\
\hline 149 & 3 & 0.73 & - & - & - & 14.9 & & & & & & & \\
\hline 180 & 2 & -1.21 & - & -. & - & 13.3 & & & & & & & \\
\hline 190 & 4 & -0.12 & - & 14.2 & - & -- & & & & & & & \\
\hline 193 & 0 & 2.06 & - & 16 & - & -- & & & & & & & \\
\hline 198 & 1 & -1.57 & - & -- & - & 13 & & & & & & & \\
\hline 212 & 0 & -2.30 & - & -- & 12.4 & -. & & & & & & & \\
\hline 227 & 2 & -1.33 & $=$ & $=$ & 13.2 & $=$ & & & & & & & \\
\hline 234 & 4 & 0.36 & -- & - & 14.6 & $=$ & & & & & & & \\
\hline 245 & 1 & 1.62 & -- & - & - & 15.64 & & & & & & & \\
\hline 247 & 4 & -0.48 & - & - & - & 13.9 & & & & & & & \\
\hline 255 & 4 & 0.24 & -- & $=$ & - & 14.5 & & & & & & & \\
\hline 256 & 0 & 4.48 & $=$ & $-\cdot$ & 18 & - & & & & & & & \\
\hline
\end{tabular}


Table 11. Statistical summary of reported data for standard reference sampie T-169 (trace constituents) -- continued

T-169 IRON (Fe) in $\mu \mathrm{g} / \mathrm{L}$

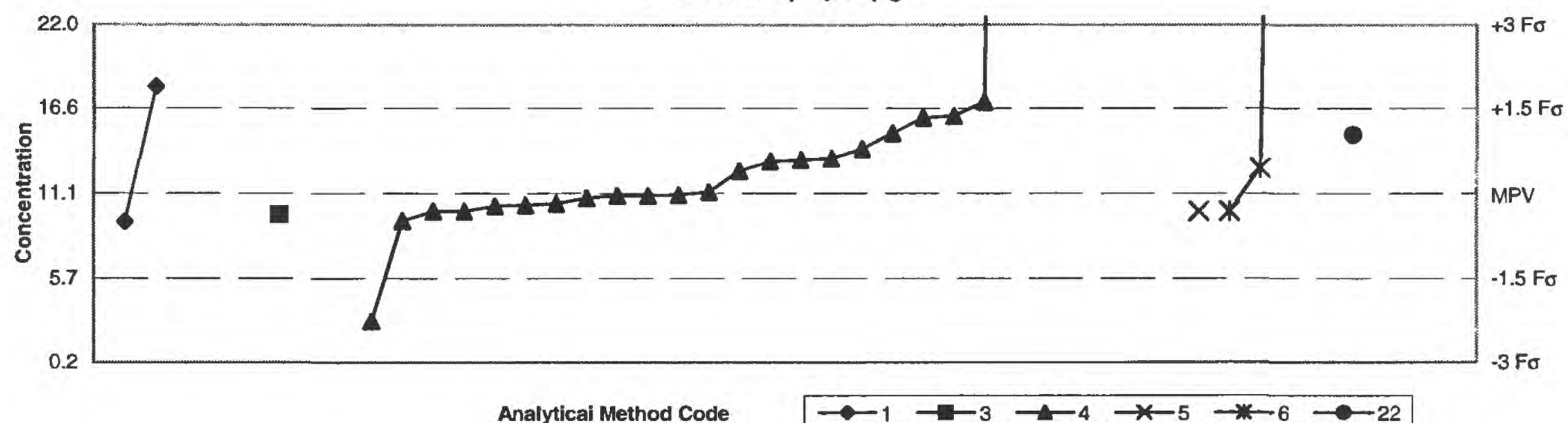

\begin{tabular}{|c|c|c|c|c|c|c|c|c|c|}
\hline \multirow[t]{2}{*}{ SUMMARY } & \multicolumn{7}{|c|}{ Methods } & \multicolumn{2}{|c|}{ Statistics } \\
\hline & 1 & 3 & 4 & 5 & 6 & 22 & Method Codes & & \\
\hline$n=$ & 2 & 1 & 22 & 1 & 3 & 1 & 01 Atomic absorption: direct, air & MPV $=$ & $11.1 \mu \mathrm{g} / \mathrm{L}$ \\
\hline Minimum $=$ & 9.34 & 9.8 & 2.9 & 10 & 10 & 14.9 & 03 Atomic absorption: graphite fumace & Josigma $=$ & 3.6 \\
\hline Maximum $=$ & 18 & & 138 & & 111.8 & & 04 Inductively coupled plasma & $n=$ & 30 \\
\hline Median $=$ & & & 11.1 & & & & 05 Diract current plasma & $\mathrm{Uh}=$ & 14.9 \\
\hline F-pseudosigma $=$ & & & 2.7 & & & & $\begin{array}{l}06 \text { Inductively coupled plasma/mass spectrometry } \\
22 \text { Colorimetric }\end{array}$ & $\mathrm{Lh}=$ & 10.0 \\
\hline
\end{tabular}

\begin{tabular}{|c|c|c|c|c|c|c|c|c|c|c|c|c|c|c|c|c|c|}
\hline \multirow[b]{2}{*}{ Lab } & \multicolumn{8}{|c|}{ Method Codes } & \multirow[b]{2}{*}{ Lab } & \multirow[b]{2}{*}{ Rating } & \multirow[b]{2}{*}{ Z-value } & \multicolumn{6}{|c|}{ Method Codes } \\
\hline & Rating & Z-value & 1 & 3 & 4 & 5 & 6 & 22 & & & & 1 & 3 & 4 & 5 & 6 & 22 \\
\hline 1 & 4 & -0.03 & $\cdots$ & - & 11.05 & $\overline{-}$ & - & $=$ & 372 & 4 & -0.04 & $\overline{-}$ & $\overline{--}$ & 11 & - & $=$ & - \\
\hline 5 & 3 & 0.62 & -- & - & 13.4 & - & - & - & & & & & & & & & \\
\hline 7 & 3 & 0.59 & - & - & 13.3 & - & - & - & & & & & & & & & \\
\hline 8 & NR & - & -- & - & $<50$ & - & - & - & & & & & & & & & \\
\hline 10 & 1 & 1.89 & 18 & - & + & -- & - & - & & & & & & & & & \\
\hline 16 & 4 & -0.32 & - & - & 10 & - & +- & -- & & & & & & & & & \\
\hline 21 & 2 & 1.03 & - & - & -- & - & - & 14.9 & & & & & & & & & \\
\hline 23 & 4 & 0.03 & - & - & 11.24 & - & - & - & & & & & & & & & \\
\hline 25 & 2 & 1.34 & - & - & 16 & - & - & $\cdots$ & & & & & & & & & \\
\hline 26 & 3 & 0.57 & - & - & 13.2 & - & - & - & & & & & & & & & \\
\hline 33 & 4 & -0.32 & -- & - & - & 10 & - & - & & & & & & & & & \\
\hline 42 & 2 & 1.36 & - & -- & t6.1 & - & + & - & & & & & & & & & \\
\hline 45 & 4 & 0.46 & -- & - & -- & - & 12.8 & - & & & & & & & & & \\
\hline 59 & NR & -- & -- & - & - & - & $<100$ & -- & & & & & & & & & \\
\hline 70 & NR &.- & $<20$ & -- & - & - & -- & - & & & & & & & & & \\
\hline 89 & 4 & -0.37 & + & 9.8 & - & - & $=$ & $\cdots$ & & & & & & & & & \\
\hline 97 & 0 & -2.57 & - & $<1.8$ & - & - & - & - & & & & & & & & & \\
\hline 105 & NR & -- & - & -- & $<20.0$ & - & - & - & & & & & & & & & \\
\hline 109 & NR & - & $<30$ & - & -- & - & -- & - & & & & & & & & & \\
\hline 113 & 4 & -0.04 & - & $=$ & 11 & - & - & $=$ & & & & & & & & & \\
\hline 121 & 2 & 1.06 & - & - & 15 & - & - & - & & & & & & & & & \\
\hline 134 & 4 & -0.22 & -- & -- & 10.35 & - & - & - & & & & & & & & & \\
\hline 138 & 4 & -0.21 & - & - & 10.4 & - & - & - & & & & & & & & & \\
\hline 146 & NR & - & -- & - & $<50.0$ & - & - & -- & & & & & & & & & \\
\hline 149 & 0 & 27.71 & - & - & -- & - & 111.8 & - & & & & & & & & & \\
\hline 180 & 4 & .0 .18 & - & - & 10.5 & -- & $=$ & $=$ & & & & & & & & & \\
\hline 190 & 4 & -0.50 & 9.34 & - & -. & - & - & - & & & & & & & & & \\
\hline 193 & NR & - & -- & $<100$ & -- & - & - & - & & & & & & & & & \\
\hline 198 & 1 & 1.61 & - & - & 17 & - & -. & - & & & & & & & & & \\
\hline 212 & NR & - & - & - & $<100$ & $=$ & $=$ & - & & & & & & & & & \\
\hline 234 & 4 & 0.40 & - & - & 12.6 & -- & - & - & & & & & & & & & \\
\hline 255 & 3 & 0.79 & - & -- & 14 & - & - & - & & & & & & & & & \\
\hline 256 & 4 & -0.08 & - & - & 10.85 & - & - & - & & & & & & & & & \\
\hline 259 & 4 & -0.48 & - & -- & 9.4 & - & -- & - & & & & & & & & & \\
\hline 265 & 4 & -0.32 & $=$ & .- & - & - & 10 & -- & & & & & & & & & \\
\hline 305 & 4 & -0.32 & - & 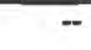 & 10 & - & - & - & & & & & & & & & \\
\hline 315 & 0 & 34.92 & $*$ & - & 138 & - & - & $\cdots$ & & & & & & & & & \\
\hline 328 & 0 & -2.27 & -- & - & 2.9 & - & - & - & & & & & & & & & \\
\hline 330 & NR & - & $<50$ & - & - & - & - & - & & & & & & & & & \\
\hline 370 & NR & - & - & - & $<50.0$ & - & - & - & & & & & & & & & \\
\hline
\end{tabular}


Table 11. Statistical summary of reported data for standard reference sample T-169 (trace constituents) -- continued

T-169 POTASSIUM (K) in mg/L

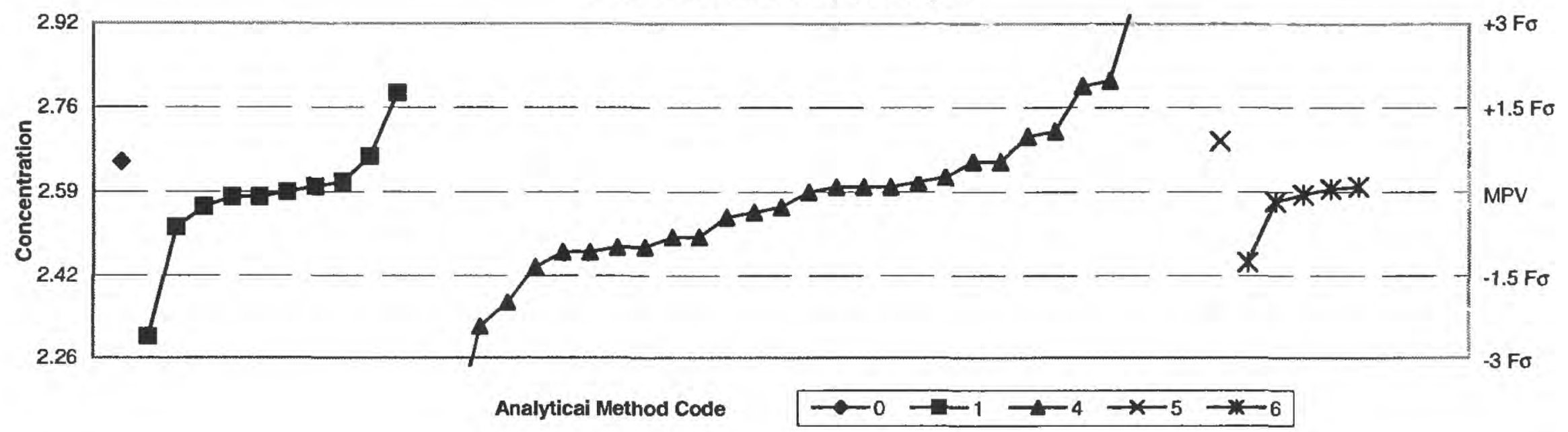

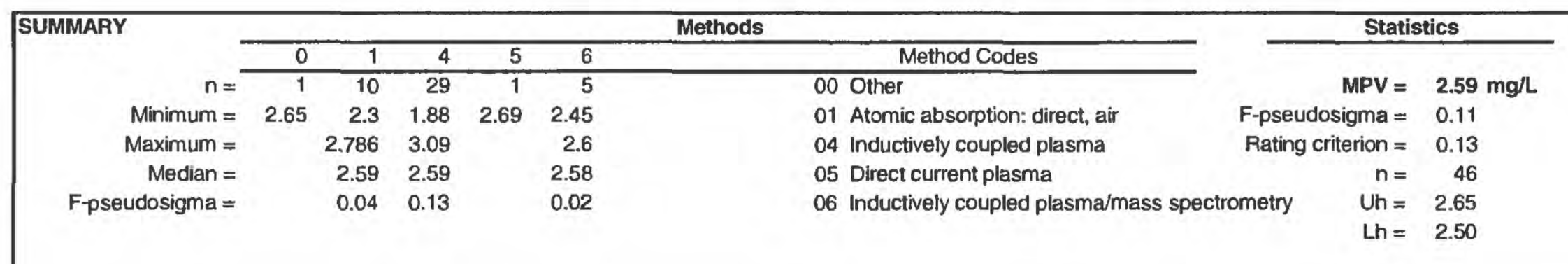

\begin{tabular}{|c|c|c|c|c|c|c|c|c|c|c|c|c|c|c|c|}
\hline \multicolumn{11}{|c|}{ Method Codes } & \multicolumn{5}{|c|}{ Method Codes } \\
\hline Lab & Rating & Z-value & 0 & 1 & 4 & 5 & 6 & Lab & Ratıng & Z-value & 0 & 1 & 4 & 5 & 6 \\
\hline 1 & 4 & 0.08 & -- & 2.6 & - & - & -- & 315 & 4 & 0.08 & - & -- & 2.6 & - & 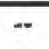 \\
\hline 5 & 1 & 1.70 & - & -- & 2.81 & - & -- & 328 & 3 & -0.69 & - & -- & 2.5 & - & -- \\
\hline 7 & 4 & 0.23 & - & -- & 2.62 & - & - & 330 & 4 & -0.39 & - & $\cdots$ & 2.54 & - & -. \\
\hline 8 & 3 & 0.85 & - & - & 2.7 & - & - & 356 & 3 & 0.93 & -. & - & 2.71 & - & - \\
\hline 16 & 4 & 0.08 & - & -- & 2.6 & - & - & 370 & 0 & 3.86 & -. & 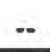 & 3.09 & - & -. \\
\hline 23 & 4 & 0.00 & $=$ & 2.59 & - & - & -- & 372 & 0 & -5.48 & - & - & 1.88 & - & - \\
\hline 24 & 4 & -0.31 & -. & - & 2.55 & - & - & & & & & & & & \\
\hline 25 & 3 & -0.93 & - & - & 2.47 & - & - & & & & & & & & \\
\hline 26 & 3 & -0.85 & -. & -- & 2.48 & - & -- & & & & & & & & \\
\hline 32 & 2 & -1.08 & - & - & -- & -- & 2.45 & & & & & & & & \\
\hline 33 & 3 & 0.77 & -. & - & -- & 2.69 & -- & & & & & & & & \\
\hline 42 & 0 & -2.08 & -- & -- & 2.32 & - & -- & & & & & & & & \\
\hline 45 & 4 & -0.15 & $\ldots$ & -. & - & -. & 2.57 & & & & & & & & \\
\hline 59 & 4 & -0.08 & -. & 2.58 & -. & - & -. & & & & & & & & \\
\hline 64 & 4 & -0.08 & -. & 2.58 & -- & - & - & & & & & & & & \\
\hline 70 & 4 & 0.00 & - & -- & 2.59 & - & $\quad-$ & & & & & & & & \\
\hline 76 & 4 & 0.05 & -- & -. & -- & -- & 2.596 & & & & & & & & \\
\hline 86 & 4 & 0.46 & - & -- & 2.65 & - & -- & & & & & & & & \\
\hline 89 & 4 & 0.15 & - & 2.61 & -- & - & -. & & & & & & & & \\
\hline 97 & 3 & -0.54 & -. & 2.52 & -. & - & - & & & & & & & & \\
\hline 105 & 4 & 0.46 & $-\cdot$ & - & 2.65 & - & - & & & & & & & & \\
\hline 109 & 0 & -2.24 & -. & 2.3 & -- & -- & - & & & & & & & & \\
\hline 110 & 1 & 1.51 & - & 2.786 & -- & - & - & & & & & & & & \\
\hline 113 & 3 & -0.85 & - & -- & 2.48 & - & -- & & & & & & & & \\
\hline 134 & 4 & -0.23 & -. & 2.56 & -. & - & - & & & & & & & & \\
\hline 138 & 3 & -0.93 & $=$ & -. & 2.47 & - & 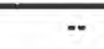 & & & & & & & & \\
\hline 142 & 2 & -1.16 & - & -. & 2.44 & -. & - & & & & & & & & \\
\hline 146 & 0 & 3.09 & - & - & 2.99 & - & -- & & & & & & & & \\
\hline 149 & 4 & 0.08 & - & - & -- & - & 2.6 & & & & & & & & \\
\hline 180 & 3 & -0.69 & - & -. & 2.5 & - & -- & & & & & & & & \\
\hline 193 & 3 & 0.54 & - & 2.66 & - & - & 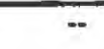 & & & & & & & & \\
\hline 198 & 4 & 0.15 & - & -. & 2.61 & -. & -- & & & & & & & & \\
\hline 212 & 1 & 1.62 & -- & - & 2.8 & -- & - & & & & & & & & \\
\hline 234 & 4 & -0.23 & - & -. & 2.56 & -. & -. & & & & & & & & \\
\hline 245 & 4 & -0.05 & - & - & - & -- & 2.584 & & & & & & & & \\
\hline 247 & 1 & -1.70 & - & - & 2.37 & $=$ & - & & & & & & & & \\
\hline 259 & 0 & -3.86 & -- & - & 2.09 & - & $-\cdot$ & & & & & & & & \\
\hline 265 & 4 & 0.08 & -- & -- & 2.6 & - & - & & & & & & & & \\
\hline 279 & 4 & 0.46 & 2.65 & - & - & -. & - & & & & & & & & \\
\hline 305 & 0 & 3.86 & - & - & 3.09 & - & - & & & & & & & & \\
\hline
\end{tabular}


Table 11. Statistical summary of reported data for standard reference sample T-169 (trace constituents) -- continued

T-169 LITHIUM (LI) In $\mu$ g/L

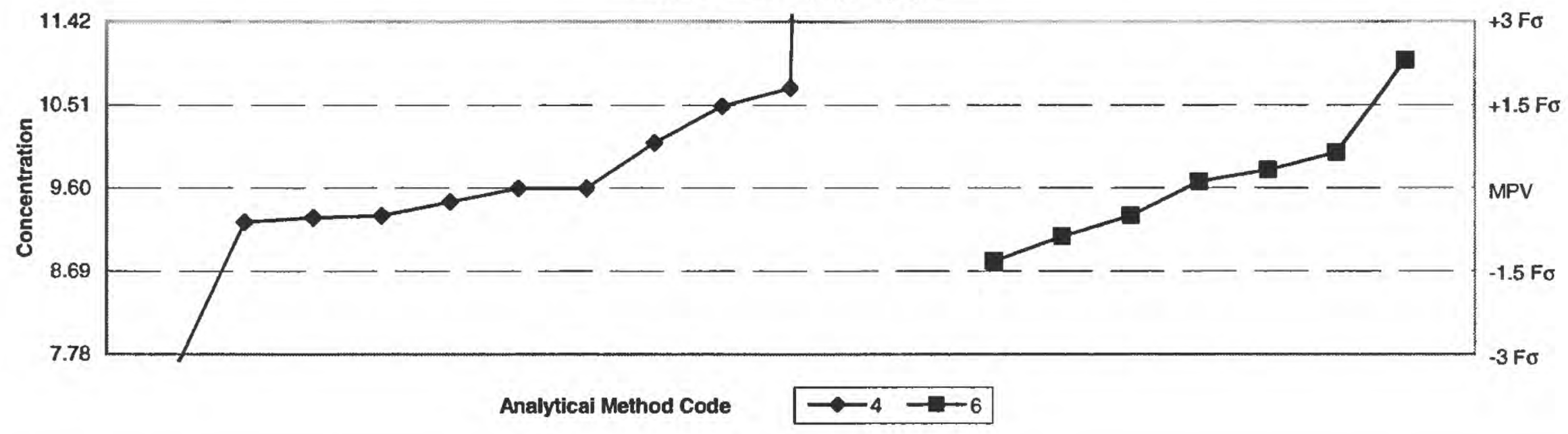

\begin{tabular}{|c|c|c|c|c|}
\hline \multirow[t]{2}{*}{ SUMMARY } & \multicolumn{3}{|c|}{ Methods } & Statistics \\
\hline & 4 & 6 & Method Codes & \\
\hline$n=$ & 11 & 7 & 04 Inductively coupled plasma & $9.60 \mu \mathrm{g} / \mathrm{L}$ \\
\hline Minimum $=$ & 7.61 & 8.8 & 06 inductively coupled plasma/mass spectrometry & F-pseudosigma $=$ \\
\hline Maximum $=$ & 42 & 11 & & $n=$ \\
\hline Median $=$ & 9.60 & 9.67 & & $U h=10.10$ \\
\hline F-pseudosigma $=$ & 0.75 & 0.53 & & $\mathrm{Lh}=9.28$ \\
\hline
\end{tabular}

\begin{tabular}{|c|c|c|c|c|c|}
\hline \multirow[b]{2}{*}{ Lab } & \multirow[b]{2}{*}{ Rating } & \multirow[b]{2}{*}{ Z-value } & \multirow{2}{*}{\multicolumn{3}{|c|}{ Method Codes }} \\
\hline & & & & & \\
\hline 1 & 3 & 0.64 & -- & 9.99 & \\
\hline 5 & 3 & -0.61 & 9.23 & - & \\
\hline 7 & 4 & -0.49 & 9.3 & .. & \\
\hline 8 & 4 & -0.49 & .- & 9.3 & \\
\hline 25 & 0 & -9.21 & $<4$ & -- & \\
\hline 26 & 0 & -3.27 & 7.61 & - & \\
\hline 32 & 2 & -1.32 & .- & 8.8 & \\
\hline 42 & 3 & -0.53 & 9.28 & -- & \\
\hline 59 & 3 & -0.87 & -. & 9.07 & \\
\hline 86 & 4 & 0.00 & 9.6 & -. & \\
\hline 105 & 0 & 53.30 & 42 & - & \\
\hline 134 & 4 & 0.00 & 9.6 & -- & \\
\hline 142 & 4 & -0.25 & 9.45 & -. & \\
\hline 234 & 3 & 0.82 & 10.1 & -. & \\
\hline 247 & 4 & 0.12 & .. & 9.67 & \\
\hline 256 & 2 & 1.48 & 10.5 & - & \\
\hline 259 & 1 & 1.81 & 10.7 & - & \\
\hline 265 & 4 & 0.33 & .. & 9.8 & \\
\hline 328 & 0 & 2.30 &.- & 11 & \\
\hline
\end{tabular}


Table 11. Statistical summary of reported data for standard reference sample T-169 (trace constituents) -- continued

T-169 MAGNESIUM (Mg) in $\mathrm{mg} / \mathrm{L}$

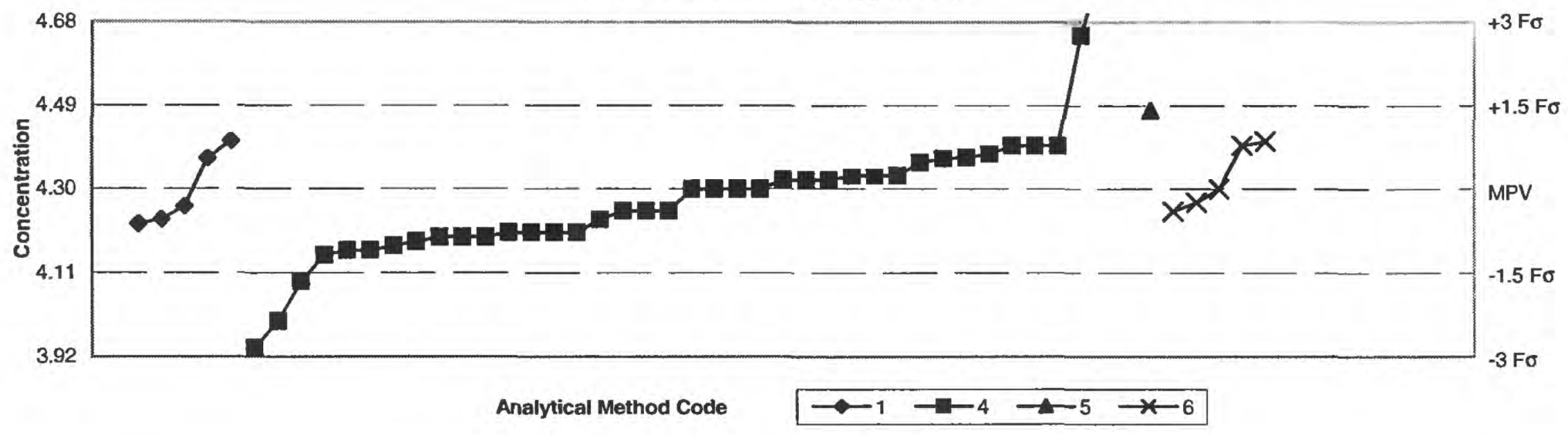

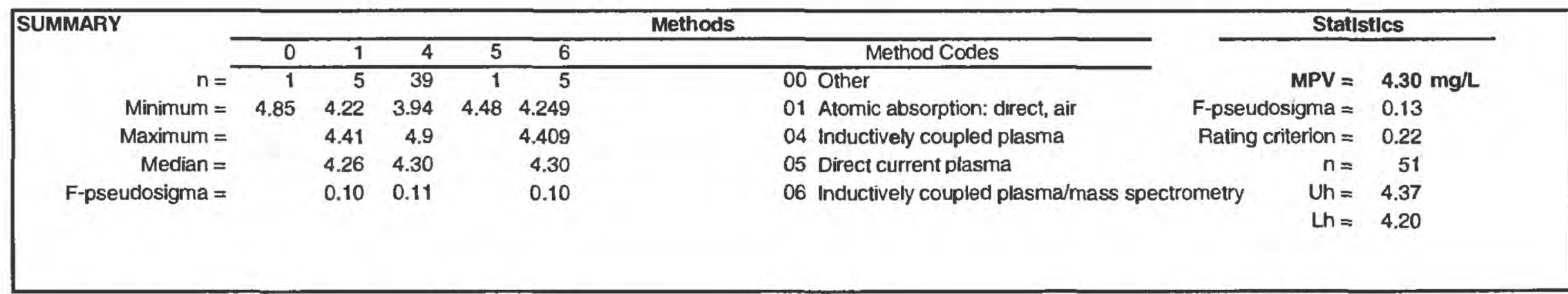

\begin{tabular}{|c|c|c|c|c|c|c|c|c|c|c|c|c|c|c|c|}
\hline \multicolumn{11}{|c|}{ Method Codes } & \multicolumn{5}{|c|}{ Method Codes } \\
\hline Lab & Rating & Z-value & 0 & 1 & 4 & 5 & 6 & Lab & Rating & Z-value & 0 & 1 & 4 & 5 & 6 \\
\hline 1 & 3 & -0.65 & - & - & 4.16 & 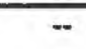 & - & 247 & 1 & -1.67 & $\cdots$ & $\cdots$ & 3.94 & - & - \\
\hline 5 & 4 & -0.47 & -. & - & 4.2 & -- & - & 259 & 4 & 0.00 & - & - & 4.3 & -. & - \\
\hline 7 & 3 & -0.51 & - & - & 4.19 & - & -- & 265 & 4 & -0.47 & -. & -- & 4.2 & - & - \\
\hline 8 & 4 & 0.47 & - & -- & 4.4 & - & - & 279 & 0 & 2.56 & 4.85 & $\cdots$ & - & - & - \\
\hline 12 & 0 & 2.79 & - & -- & 4.9 & $\ldots$ & - & 305 & 4 & 0.37 & -. & -. & 4.38 & - & $=$ \\
\hline 16 & 4 & 0.47 & - & -- & 4.4 & - & - & 315 & 4 & -0.47 & $\cdots$ & $\bar{*}$ & 4.2 & - & - \\
\hline 23 & 4 & -0.37 & - & 4.22 & -. & .. & - & 328 & 4 & 0.00 & - & - & 4.3 & .. & - \\
\hline 24 & 3 & -0.65 & - & - & 4.16 & -. & -- & 330 & 4 & -0.33 & - & - & 4.23 & - & - \\
\hline 25 & 4 & 0.33 & - & - & 4.37 & - & .- & 356 & 1 & 1.63 & - & -- & 4.65 & - & - \\
\hline 26 & 4 & 0.09 & - &.- & 4.32 & - & -- & 370 & 4 & -0.23 & - & $\because$ & 4.25 & - & - \\
\hline 32 & 4 & 0.00 & - & $\ldots$ & - & - & 4.3 & 372 & 3 & -0.98 & $\cdots$ & 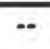 & 4.09 & -- & - \\
\hline 33 & 3 & 0.84 & - & - & - & 4.48 & - & & & & & & & & \\
\hline 42 & 2 & -1.40 & - & $\cdots$ & 4 & - & - & & & & & & & & \\
\hline 45 & 4 & -0.14 & - & -. & -- & - & 4.27 & & & & & & & & \\
\hline 46 & 3 & -0.51 & - & - & 4.19 & -. & - & & & & & & & & \\
\hline 59 & 4 & -0.19 & - & 4.26 & - & - & - & & & & & & & & \\
\hline
\end{tabular}


Table 11. Statistical summary of reported data for standard reference sample T-169 (trace constituents) -- continued

T-169 MANGANESE (Mn) in $\mu \mathrm{g} / \mathrm{L}$

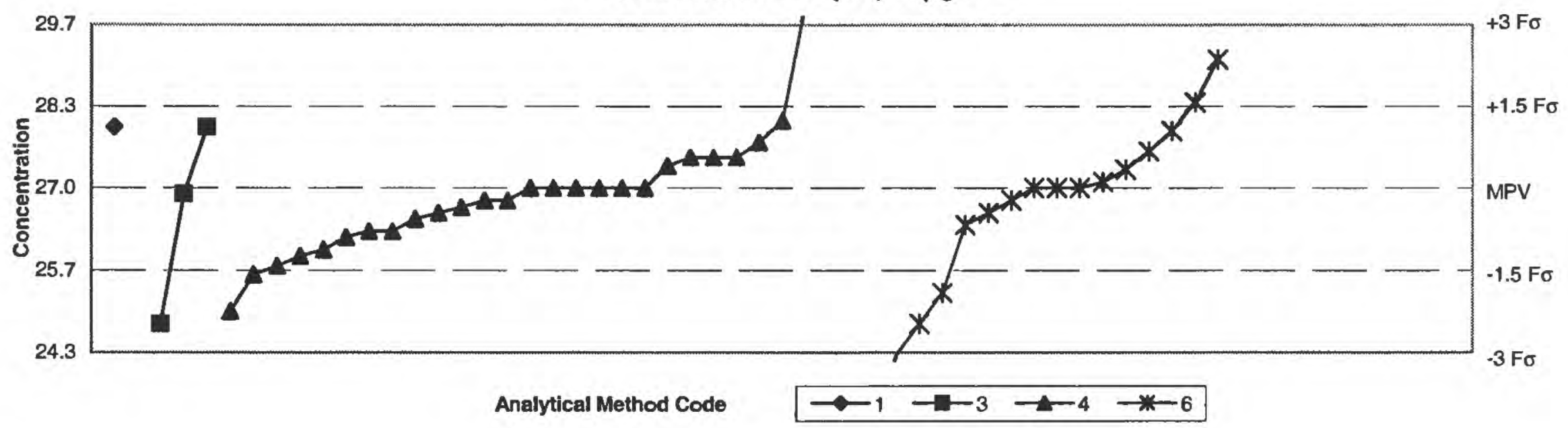

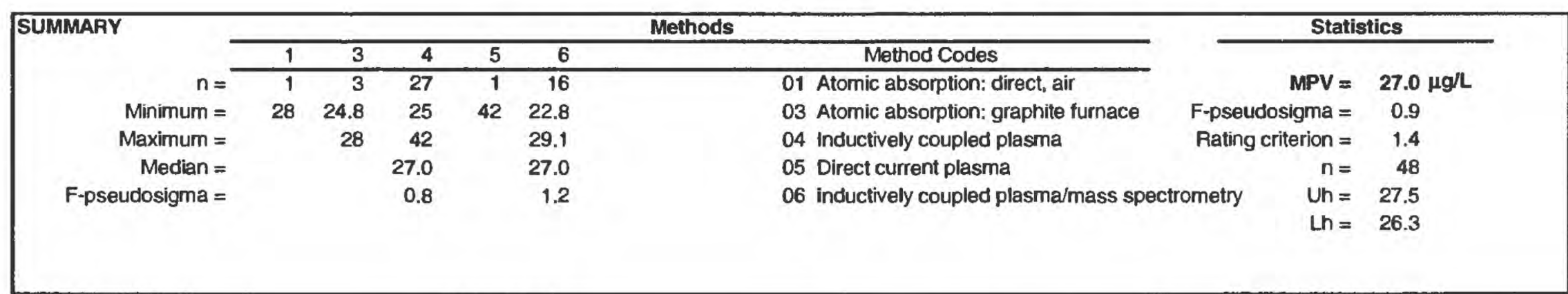

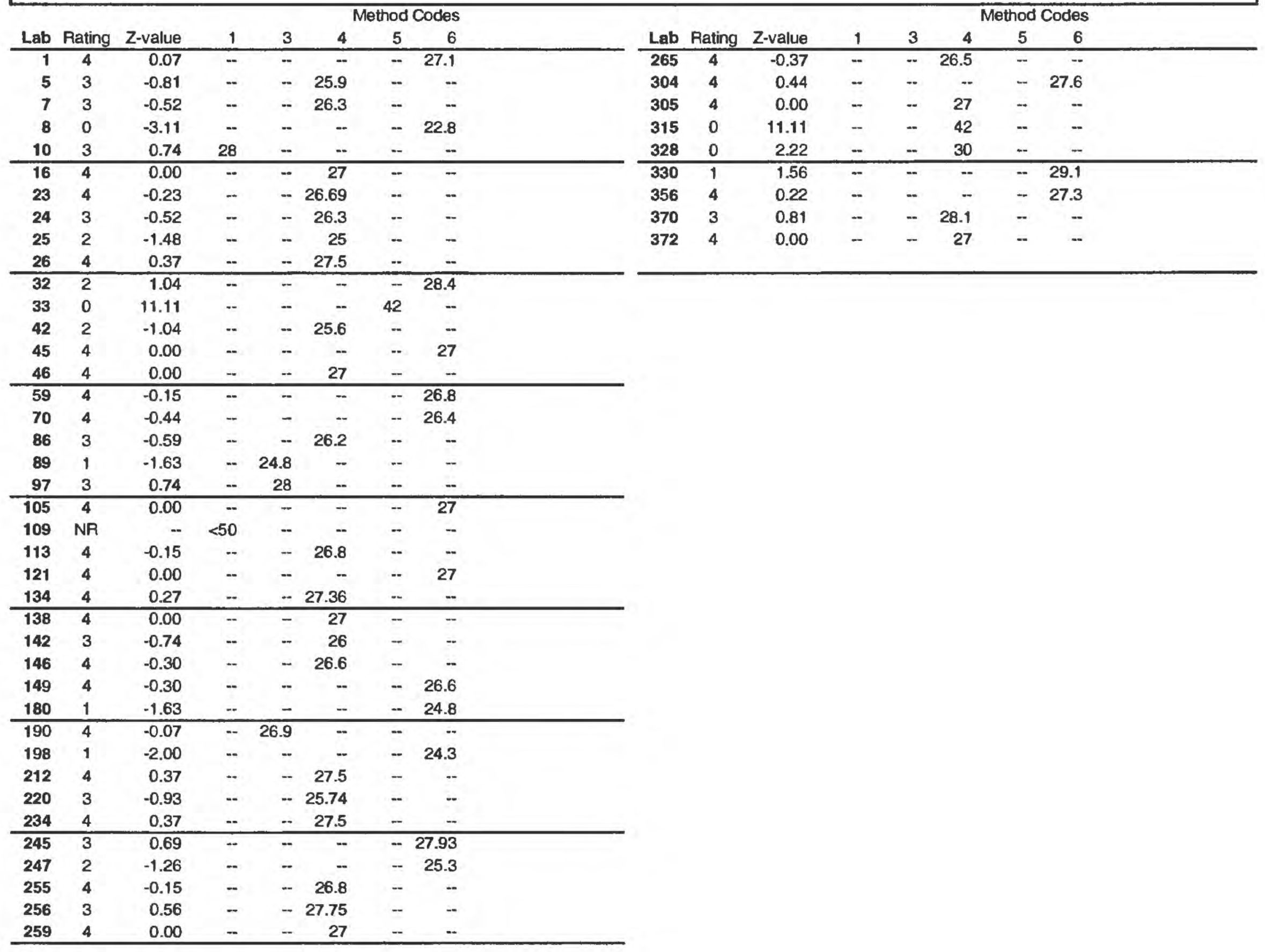


Table 11. Statistical summary of reported data for standard reference sample T-169 (trace constituents) -- continued

T-169 MOLYBDENUM (Mo) in $\mu \mathrm{g} / \mathrm{L}$

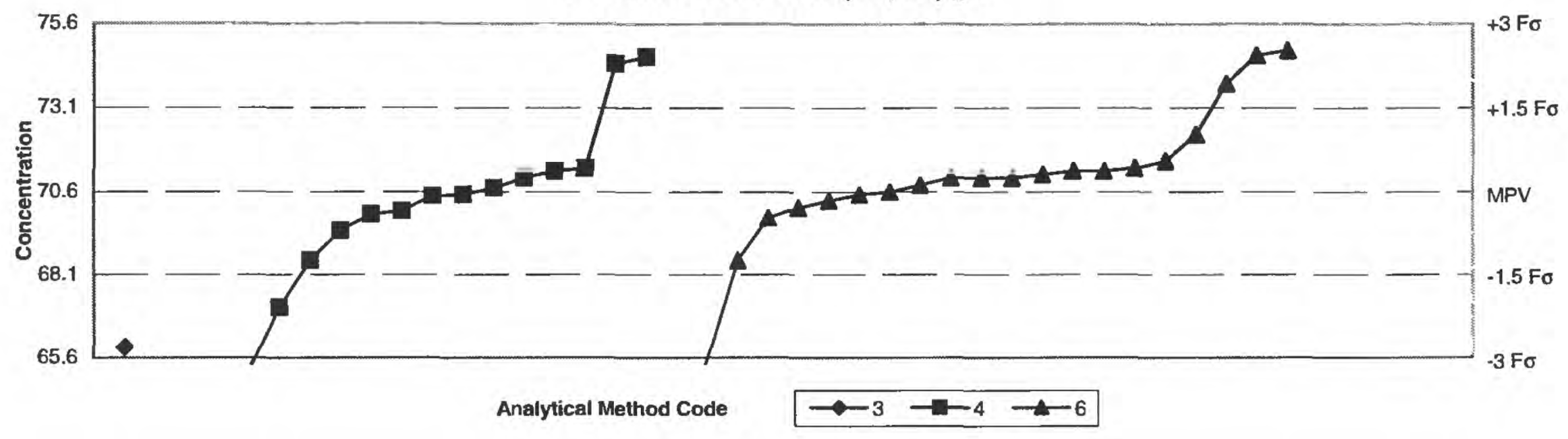

\begin{tabular}{|c|c|c|c|c|c|c|}
\hline \multirow[t]{2}{*}{ SUMMARY } & \multicolumn{4}{|c|}{ Methods } & \multicolumn{2}{|c|}{ Statistics } \\
\hline & 3 & 4 & 6 & Method Codes & & \\
\hline$n=$ & 1 & 17 & 21 & 03 Atomic absorption: graphite furnace & MPV $=$ & $70.6 \mu \mathrm{g} / \mathrm{L}$ \\
\hline Minimum = & 65.9 & 61 & 62.6 & 04 Inductively coupled plasma & F-pseudosigma $=$ & 1.7 \\
\hline Maximum = & & 74.6 & 74.8 & 06 Inductively coupled plasma/mass spectrometry & Rating criterion = & 3.5 \\
\hline Median $=$ & & 70.0 & 71.0 & & $n=$ & 39 \\
\hline \multirow[t]{2}{*}{ F.pseudosigma $=$} & & 2.9 & 0.7 & & Uh $=$ & 71.2 \\
\hline & & & & & Lh $=$ & 69.0 \\
\hline
\end{tabular}

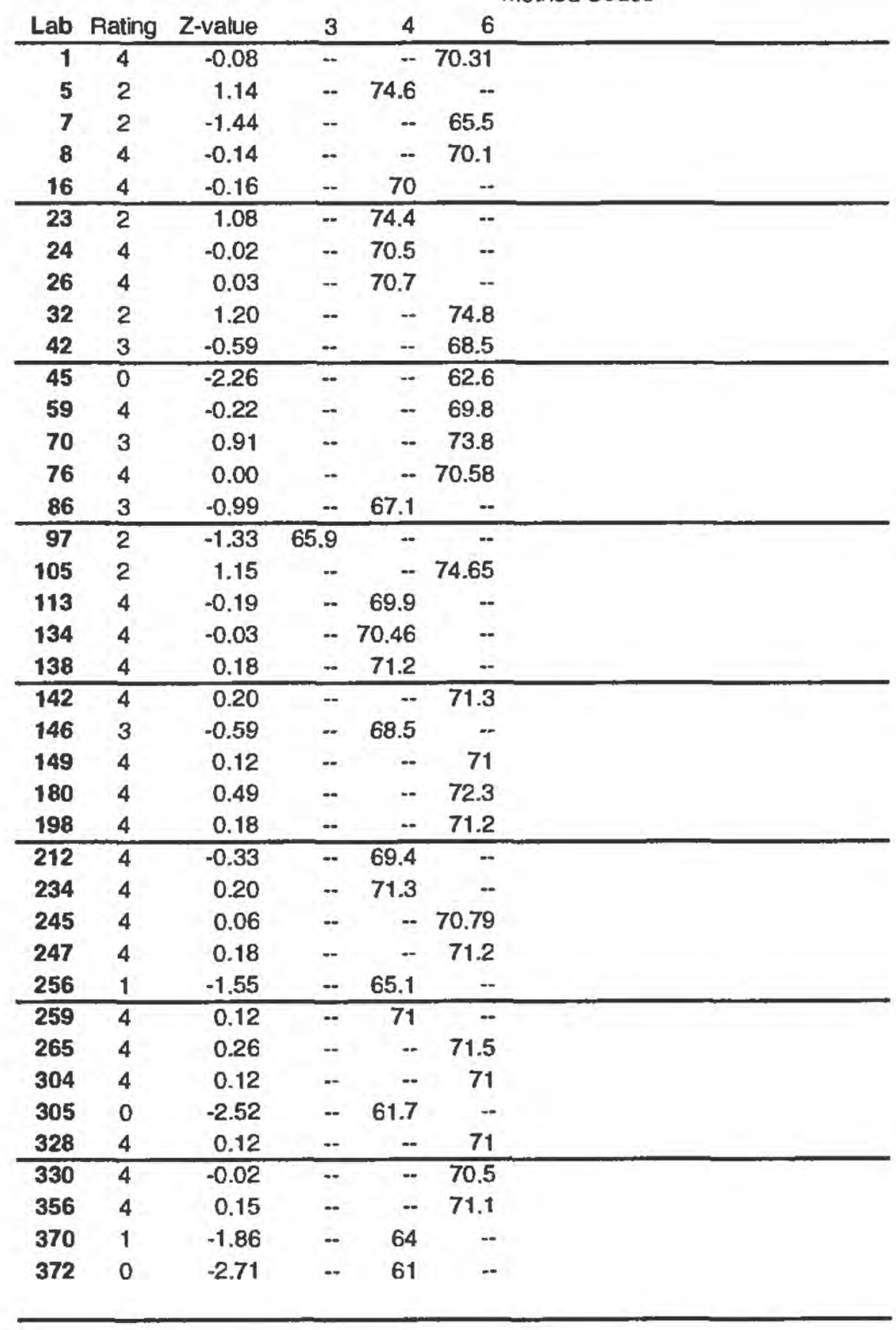


Table 11. Statistical summary of reported data for standard reference aample T-169 (trace constituents) -- continued

T-169 SODIUM ( $\mathrm{Na}$ ) in $\mathrm{mg} / \mathrm{L}$

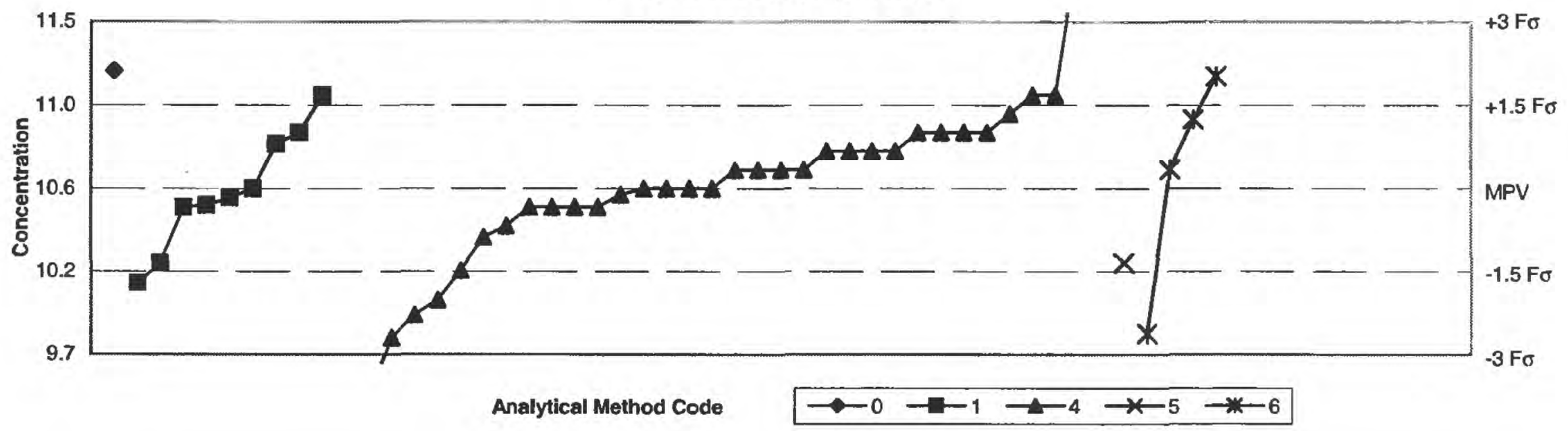

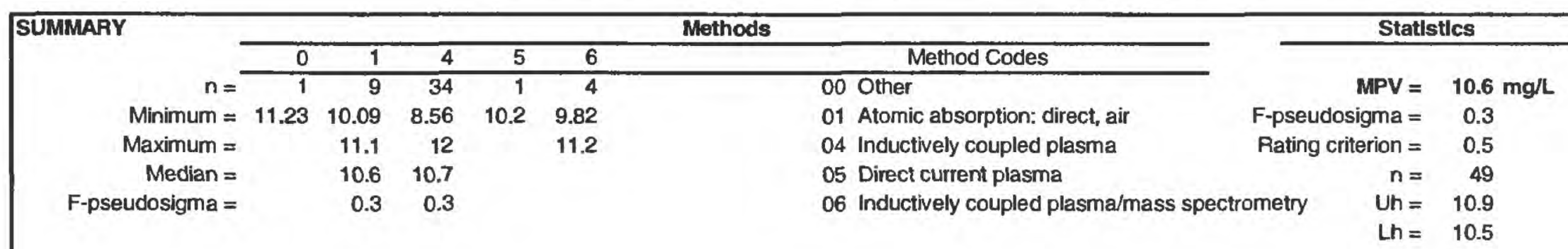

\begin{tabular}{|c|c|c|c|c|c|c|c|c|c|c|c|c|c|c|c|}
\hline \multicolumn{11}{|c|}{ Method Codes } & \multicolumn{5}{|c|}{ Method Codes } \\
\hline Lab & Rating & Z-value & 0 & 1 & 4 & 5 & 6 & Lab & Rating & Z-value & 0 & 1 & 4 & 5 & 6 \\
\hline 1 & 3 & -0.83 & 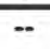 & 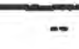 & 10.16 & $\cdots$ & $\cdots$ & 265 & 4 & -0.19 & 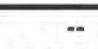 & $=$ & 10.5 & - & - \\
\hline 5 & 4 & 0.38 & - & -- & 10.8 & -- & - & 279 & 2 & 1.19 & 11.23 & -- & -- & - & - \\
\hline 7 & 4 & -0.19 & - & - & 10.5 & -. & - & 305 & 3 & 0.57 & - & -- & 10.9 & - & - \\
\hline 8 & 4 & 0.00 & -- & -- & 10.6 & - & - & 315 & 4 & 0.00 & - & -. & 10.6 & - & - \\
\hline 12 & 0 & 2.64 & - & - & 12 & $-\cdot$ & - & 328 & 3 & 0.75 & - & $\ldots$ & 11 & - & - \\
\hline 16 & 2 & -1.13 & $\cdots$ & - & 10 & $=$ & - & 330 & 4 & 0.19 & -- & -- & 10.7 & - & -- \\
\hline 23 & 4 & -0.19 & $\cdots$ & 10.5 & - & -. & - & 356 & 3 & 0.94 & - & - & 11.1 & - & -. \\
\hline 24 & 4 & -0.19 & -- & - & 10.5 & $*$ & -- & 370 & 0 & 2.45 & - & - & 11.9 & - & -- \\
\hline 25 & 2 & -1.28 & - & -- & 9.92 & -. & -- & 372 & 0 & -3.85 & -- & -. & 8.56 & - & - \\
\hline 26 & 4 & 0.19 & - & .. & 10.7 & - & - & & & & & & & & \\
\hline 32 & 2 & -1.47 & $\overline{-}$ & - & 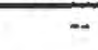 & $\cdots$ & 82 & & & & & & & & \\
\hline
\end{tabular}


Table 11. Statistical summary of reported data for standard reference sample T-169 (trace constituents) -- continued

T-169 NICKEL (Ni) in $\mu \mathrm{g} / \mathrm{L}$

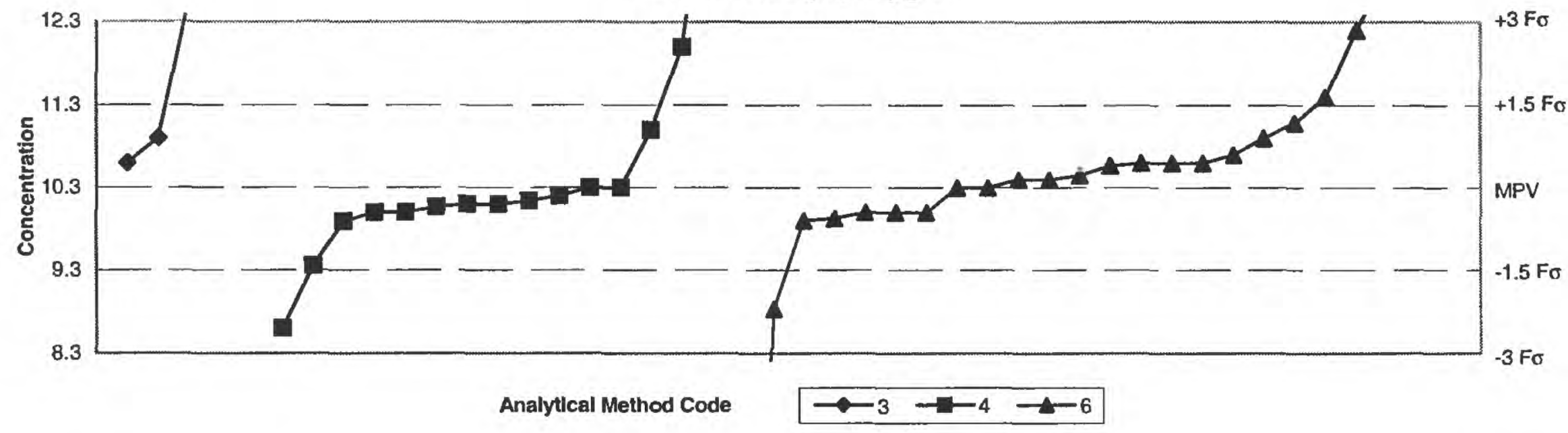

\begin{tabular}{|c|c|c|c|c|c|c|}
\hline \multirow[t]{2}{*}{ SUMMARY } & \multicolumn{4}{|c|}{ Methods } & \multicolumn{2}{|c|}{ Statistics } \\
\hline & 3 & 4 & 6 & Method Codes & & \\
\hline$n=$ & 4 & 15 & 22 & 03 Atomic absorption: graphite furnace & MPV = & $10.3 \mu \mathrm{g} / \mathrm{L}$ \\
\hline Minimum = & 10.6 & 8.6 & 1.9 & 04 Inductively coupled plasma & F-pseudosigma $=$ & 0.7 \\
\hline Maximum $=$ & 23.2 & 14.6 & 12.9 & 06 Inductively coupled plasma/mass spectrometry & $\mathrm{n}=$ & 41 \\
\hline Median $=$ & & 10.1 & 10.4 & & Un $=$ & 10.9 \\
\hline F-pseudosigma $=$ & & 0.2 & 0.5 & & $\operatorname{Lh}=$ & 10.0 \\
\hline
\end{tabular}

\begin{tabular}{|c|c|c|c|c|c|c|c|c|c|c|c|c|}
\hline \multicolumn{9}{|c|}{ Method Codes } & \multirow{2}{*}{\multicolumn{4}{|c|}{$6^{\text {Method Codes }}$}} \\
\hline Lab & Rating & Z-value & 3 & 4 & 6 & Lab & Rating & Z-value & & & & \\
\hline 1 & 4 & 0.40 & - & - & 10.57 & 356 & 4 & 0.15 & - & - & 10.4 & \\
\hline 5 & 0 & 6.45 & - & 14.6 & -- & 370 & 3 & -0.61 & - & 9.89 & - & \\
\hline 7 & 0 & -12.59 & - & -- & 1.9 & 372 & 2 & 1.05 & -. & 11 & -. & \\
\hline 8 & 4 & 0.45 & - & - & 10.6 & & & & & & & \\
\hline 16 & 4 & -0.45 & - & 10 & - & & & & & & & \\
\hline 23 & 4 & -0.24 & -- & 10.14 & $\because$ & & & & & & & \\
\hline 25 & 0 & 2.55 & - & 12 & - & & & & & & & \\
\hline 32 & 3 & 0.90 & - & - & 10.9 & & & & & & & \\
\hline 42 & 4 & 0.45 & - & - & 10.6 & & & & & & & \\
\hline 45 & 4 & -0.45 & - & - & 10 & & & & & & & \\
\hline 59 & 4 & -0.45 & $\cdots$ & - & 10 & & & & & & & \\
\hline 70 & 0 & 2.85 & - & - & 12.2 & & & & & & & \\
\hline 76 & 4 & 0.22 & - & - & 10.45 & & & & & & & \\
\hline 86 & 2 & -1.42 & -- & 9.35 & -- & & & & & & & \\
\hline 89 & 3 & 0.90 & 10.9 & - & - & & & & & & & \\
\hline 97 & 0 & 3.45 & 12.6 & - & - & & & & & & & \\
\hline 105 & NR & -- & -. & -. & $<50$ & & & & & & & \\
\hline 113 & 4 & -0.30 & - & 10.1 & -. & & & & & & & \\
\hline 134 & 4 & -0.34 & - & 10.07 & -- & & & & & & & \\
\hline 138 & 4 & 0.45 & - & - & 10.6 & & & & & & & \\
\hline 142 & 4 & 0.00 & $\cdots$ & - & 10.3 & & & & & & & \\
\hline 146 & 4 & -0.30 & $*$ & 10.1 & -- & & & & & & & \\
\hline 149 & 4 & 0.00 & -- & -- & 10.3 & & & & & & & \\
\hline 180 & 0 & -2.20 & -. & - & 8.83 & & & & & & & \\
\hline 183 & 0 & 19.34 & 23.2 & - & -- & & & & & & & \\
\hline 190 & 4 & 0.45 & 10.6 & $\cdots$ & $=$ & & & & & & & \\
\hline 193 & NR & - & $<12.5$ & $\cdots$ & - & & & & & & & \\
\hline 198 & 3 & -0.55 & - & - & 9.93 & & & & & & & \\
\hline 212 & 0 & -2.55 & - & 8.6 & - & & & & & & & \\
\hline 234 & 4 & 0.00 & -- & 10.3 & - & & & & & & & \\
\hline 245 & 2 & 1.17 & - & - & 11.08 & & & & & & & \\
\hline 247 & 4 & 0.15 & - & - & 10.4 & & & & & & & \\
\hline 255 & 1 & 1.65 & - & - & 11.4 & & & & & & & \\
\hline 256 & 4 & -0.15 & - & 10.2 & - & & & & & & & \\
\hline 259 & 4 & -0.45 & - & 10 & - & & & & & & & \\
\hline 265 & 4 & -0.45 & - & - & 10 & & & & & & & \\
\hline 304 & 3 & 0.60 & -- & -- & 10.7 & & & & & & & \\
\hline 305 & 4 & 0.00 & -- & 10.3 & - & & & & & & & \\
\hline 328 & 3 & -0.60 & -- & -- & 9.9 & & & & & & & \\
\hline 330 & 0 & 3.90 & - & - & 12.9 & & & & & & & \\
\hline
\end{tabular}


Table 11. Statistical summary of reported data for standard reference sample T-169 (trace constituents) -- continued

T-169 LEAD (Pb) in $\mu \mathrm{g} / \mathrm{L}$

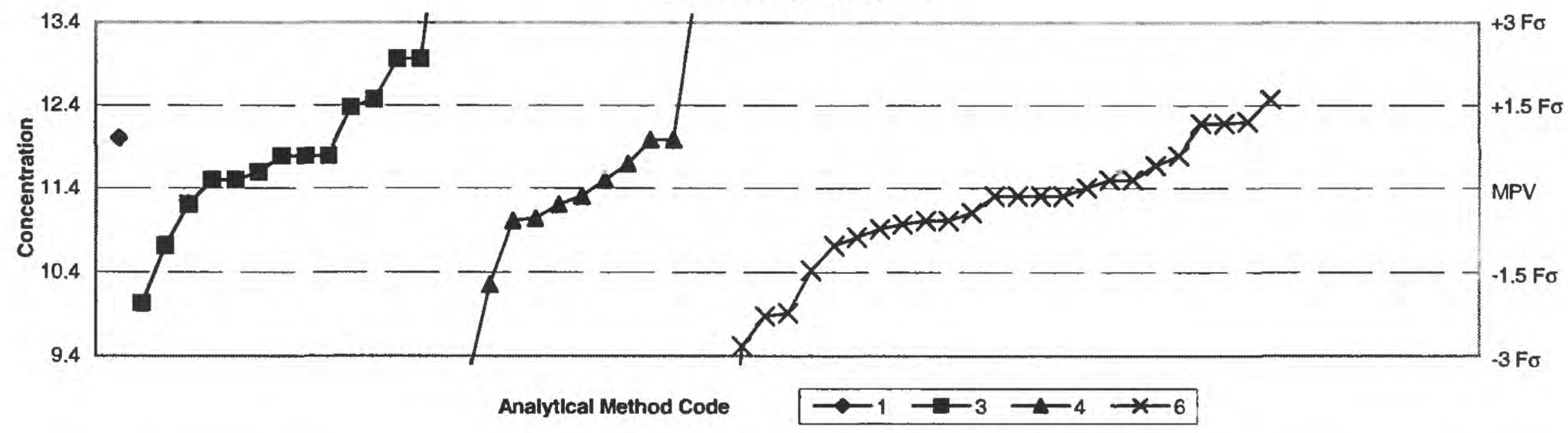

\begin{tabular}{|c|c|c|c|c|c|c|c|}
\hline \multirow[t]{2}{*}{ SUMMARY } & \multicolumn{5}{|c|}{ Methods } & \multicolumn{2}{|c|}{ Statistics } \\
\hline & 1 & 3 & 4 & 6 & Method Codes & & \\
\hline$n=$ & 1 & 14 & 11 & 25 & 01 Atomic absorption: direct. air & MPV $=$ & $11.4 \mu g^{\prime} L$ \\
\hline Minimum = & 12.02 & 10 & 9 & 5.74 & 03 Atomic absorption: graphite fumace & F-pseudosigma $=$ & 0.7 \\
\hline Maximum $=$ & & 15.1 & 13.9 & 12.5 & 04 Inductively coupled plasma & $\mathrm{n}=$ & 51 \\
\hline Median $=$ & & 11.8 & 11.3 & 11.3 & 06 Inductively coupled plasma/mass sp & ometry & 11.9 \\
\hline F-pseudosigma $=$ & & 0.7 & 0.6 & 0.5 & & $\mathrm{Lh}=$ & 11.0 \\
\hline
\end{tabular}

\begin{tabular}{|c|c|c|c|c|c|c|c|c|c|c|c|c|c|}
\hline \multicolumn{7}{|c|}{ Method Codes } & \multirow[b]{2}{*}{ Lab } & \multirow[b]{2}{*}{ Rating } & \multirow[b]{2}{*}{ Z-value } & \multicolumn{4}{|c|}{ Method Codes } \\
\hline Lab & Rating & Z-value & 1 & 3 & 4 & 6 & & & & 1 & 3 & 4 & 6 \\
\hline 1 & 4 & 0.00 & - & - & - & 11.4 & 255 & 2 & 1.17 & - & - & - & 12.2 \\
\hline 5 & 0 & -2.05 & -. & 10 & -. & -- & 256 & 3 & -0.54 & -. & -. & 11.03 & .. \\
\hline 7 & 4 & -0.15 & - & - & - & 11.3 & 259 & 4 & 0.15 & - & -- & 11.5 & -- \\
\hline 8 & 1 & 1.61 & -. & -- & - & 12.5 & 265 & 3 & -0.59 & - & -- & - & 11 \\
\hline 10 & 4 & 0.15 & -- & 11.5 & - & -- & 304 & 4 & 0.15 & - & - & - & 11.5 \\
\hline 12 & 0 & 2.35 & - & 13 & - & $\cdots$ & 305 & 3 & 0.88 & $=$ & 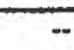 & 12 & -- \\
\hline 16 & 3 & -0.59 & -- & -. & 11 & - & 328 & 3 & -0.59 & -. & -- & -. & 11 \\
\hline 23 & 1 & -1.72 & - & -- & 10.23 & -. & 330 & 3 & 0.59 & -. & -. & - & 11.8 \\
\hline 25 & 4 & 0.15 & -- & 11.5 & - & -- & 356 & 0 & -8.30 & -. & -- & - & 5.74 \\
\hline 26 & 4 & -0.29 & -- & 11.2 & -. & -- & 370 & 3 & 0.59 & - & 11.8 & - & $=$ \\
\hline$\overline{32}$ & 2 & -1.47 & $\overline{-}$ & -. & $=$ & 10.4 & 372 & 0 & -3.52 & $=$ & - & 9 & -. \\
\hline 42 & 4 & -0.15 & - & - & - & 11.3 & & & & & & & \\
\hline 45 & 0 & -2.83 & - & -- & - & 9.47 & & & & & & & \\
\hline 46 & 3 & 0.59 & -- & 11.8 & - & -. & & & & & & & \\
\hline 59 & 2 & 1.17 & -. & -. & - & 12.2 & & & & & & & \\
\hline 70 & 0 & -2.29 & - & $\cdots$ & - & 9.84 & & & & & & & \\
\hline 76 & 4 & 0.41 & - & .. & -- & 11.68 & & & & & & & \\
\hline 86 & 4 & -0.29 & - & -. & 11.2 & -. & & & & & & & \\
\hline 89 & 4 & 0.29 & - & 11.6 & - & - & & & & & & & \\
\hline 97 & 1 & 1.61 & -- & 12.5 & -- & -. & & & & & & & \\
\hline 105 & 3 & -0.65 & - & - & - & 10.96 & & & & & & & \\
\hline 109 & 3 & 0.91 & 12.02 & -. & - & -. & & & & & & & \\
\hline 113 & 3 & 0.59 & -- & 11.8 & - & -. & & & & & & & \\
\hline 121 & 3 & -0.88 & - & -. & -- & 10.8 & & & & & & & \\
\hline 134 & 4 & 0.44 & - & - & 11.7 & - & & & & & & & \\
\hline 138 & 4 & -0.44 & $=$ & $=$ & + & 11.1 & & & & & & & \\
\hline 142 & 2 & -1.03 & -. & -. & -- & 10.7 & & & & & & & \\
\hline 146 & 3 & 0.88 & -- & -. & 12 & -- & & & & & & & \\
\hline 147 & 4 & -0.15 & - & - & - & 11.3 & & & & & & & \\
\hline 149 & 4 & 0.15 & - & - & - & 11.5 & & & & & & & \\
\hline 180 & 0 & -2.23 & - & -- & $\ldots$ & 9.88 & & & & & & & \\
\hline 183 & 0 & 5.43 & - & 15.1 & -. & -. & & & & & & & \\
\hline 190 & 2 & -1.03 & - & 10.7 & - & -. & & & & & & & \\
\hline 193 & 0 & 2.35 & -- & 13 & - & -- & & & & & & & \\
\hline 198 & 3 & -0.73 & -. & -. & -- & 10.9 & & & & & & & \\
\hline 212 & 0 & 3.67 & - & 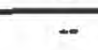 & 13.9 & -- & & & & & & & \\
\hline 227 & 4 & -0.15 & -. & -- & 11.3 & -. & & & & & & & \\
\hline 234 & 2 & 1.47 & - & 12.4 & -. & - & & & & & & & \\
\hline 245 & 2 & 1.20 & - & -. & - & 12.22 & & & & & & & \\
\hline 247 & 4 & -0.15 & -. & .. & -. & 11.3 & & & & & & & \\
\hline
\end{tabular}


Table 11. Statistical summary of reported data for standard reference sample T-169 (trace constituents) -- continued

T-169 ANTIMONY (Sb) in $\mu \mathrm{g} / \mathrm{L}$

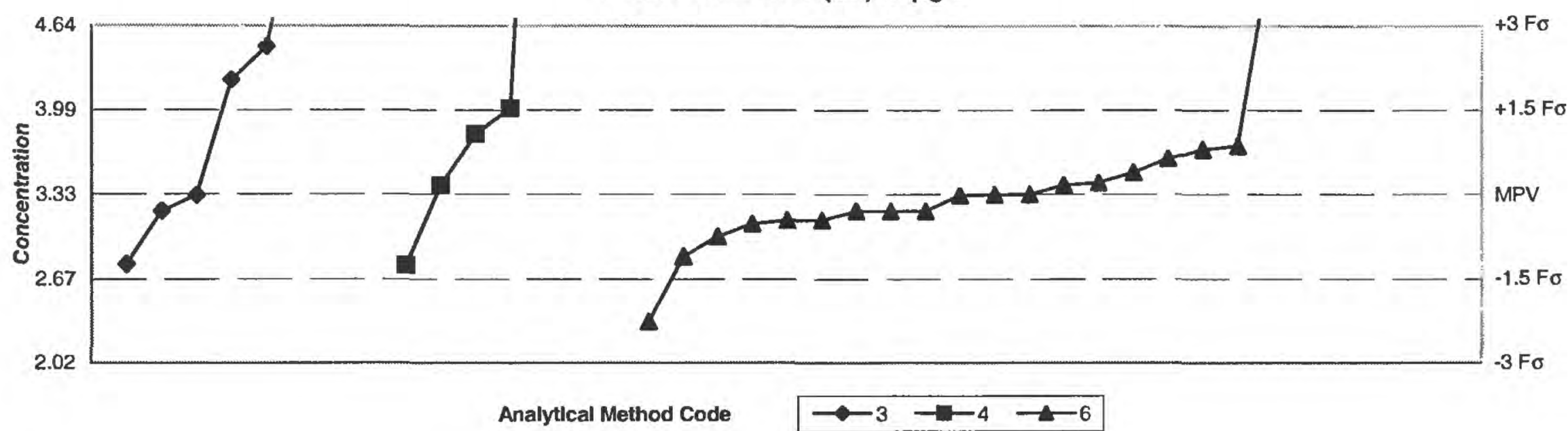

\begin{tabular}{|c|c|c|c|c|c|c|}
\hline \multirow[t]{2}{*}{ SUMMARY } & \multicolumn{4}{|c|}{ Methods } & \multicolumn{2}{|c|}{ Statistics } \\
\hline & 3 & 4 & 6 & Method Codes & & \\
\hline$n=$ & 6 & 5 & 20 & 03 Atomic absorption: graphite fumace & MPV $=$ & $3.33 \mu \mathrm{g} / \mathrm{L}$ \\
\hline Minimum $=$ & 2.79 & 2.78 & 2.34 & 04 Inductively coupled plasma & F-pseudosigma $=$ & 0.44 \\
\hline Maximum $=$ & 5.72 & 9 & 7.43 & 06 Inductively coupled plasma/mass spectrometry & $n=$ & 31 \\
\hline Median $=$ & 3.77 & 3.80 & 3.32 & & Uh $=$ & 3.76 \\
\hline F-pseudosigma $=$ & 0.95 & 0.44 & 0.32 & & $\operatorname{Lh}=$ & 3.17 \\
\hline
\end{tabular}

\begin{tabular}{|c|c|c|c|c|c|}
\hline \multirow[b]{2}{*}{ Lab } & \multirow[b]{2}{*}{ Rating } & \multirow[b]{2}{*}{$Z$-value } & \multicolumn{3}{|r|}{ Method Codes } \\
\hline & & & 3 & 4 & 6 \\
\hline 1 & 3 & 0.87 & - & -- & 3.71 \\
\hline 5 & 0 & 2.63 & 4.48 & -- & - \\
\hline 7 & 4 & 0.16 & -. & -. & 3.4 \\
\hline 8 & 4 & -0.30 & -- & -- & 3.2 \\
\hline 16 & 4 & 0.16 & .. & 3.4 & $\cdots$ \\
\hline 25 & NR & $=$ & $\because$ & $<50$ & $\cdots$ \\
\hline 32 & 3 & -0.53 & - & -. & 3.1 \\
\hline 42 & 4 & -0.46 & - & - & 3.13 \\
\hline 45 & 2 & -1.10 & - & -- & 2.85 \\
\hline 59 & NR & - &.- & - & $<5$ \\
\hline 70 & 0 & 9.37 & - & 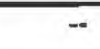 & 7.43 \\
\hline 89 & NR & -- & $<5.00$ & - & - \\
\hline 97 & 4 & -0.02 & 3.32 & - & $\cdots$ \\
\hline 105 & 4 & -0.46 & -. & - & 3.13 \\
\hline 134 & 4 & -0.30 & 3.2 & -- & -. \\
\hline 138 & 3 & -0.75 & -. & 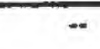 & 3 \\
\hline 142 & 4 & 0.21 & - & -- & 3.42 \\
\hline 146 & NR & -- & -. & $<20.0$ & -. \\
\hline 149 & 4 & -0.30 & -- & - & 3.2 \\
\hline 180 & 4 & -0.02 & -- & - & 3.32 \\
\hline 183 & 0 & 2.03 & 4.22 & - & - \\
\hline 193 & NR & -. & $<10$ & -- & -. \\
\hline 198 & 3 & 0.80 & -. & -- & 3.68 \\
\hline 212 & 2 & 1.07 & .. & 3.8 & - \\
\hline 234 & 2 & -1.23 & 2.79 & -- & -- \\
\hline 245 & 4 & 0.00 & - & $=$ & 3.328 \\
\hline 247 & 0 & -2.26 & -- & -- & 2.34 \\
\hline 256 & 2 & -1.26 & - & 2.78 & -- \\
\hline 265 & 4 & -0.30 & - & -- & 3.2 \\
\hline 304 & 4 & 0.00 & - & - & 3.33 \\
\hline 305 & 0 & 12.96 & - & 9 & - \\
\hline 328 & 0 & 4.50 & - & - & 5.3 \\
\hline 330 & 4 & 0.39 & -- & -. & 3.5 \\
\hline 356 & 3 & 0.64 & - & .. & 3.61 \\
\hline 370 & 0 & 5.46 & 5.72 & -. & -- \\
\hline 372 & 1 & 1.53 & -- & 4 & - \\
\hline
\end{tabular}


Table 11. Statistical summary of reported data for standard reference sample T-169 (trace constituents) -- continued

T-169 SELENIUM (Se) in $\mu \mathrm{g} / \mathrm{L}$

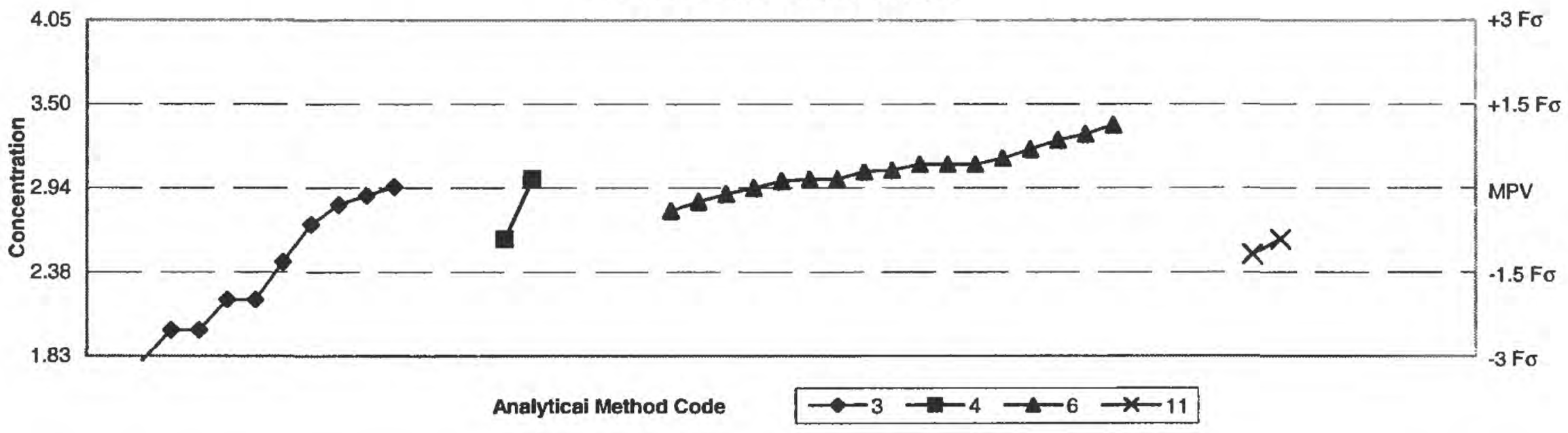

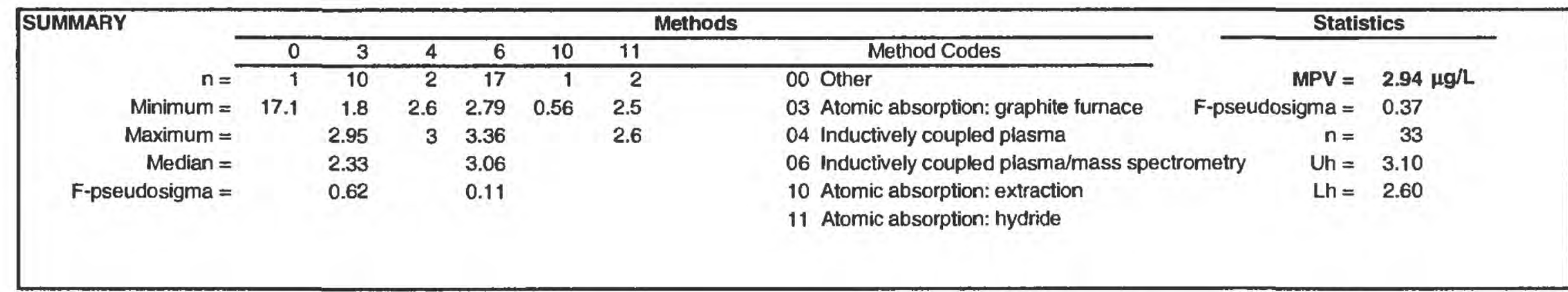

\begin{tabular}{|c|c|c|c|c|c|c|c|c|}
\hline \multirow[b]{2}{*}{ Lab } & \multirow[b]{2}{*}{ Rating } & \multirow[b]{2}{*}{$Z$-value } & \multicolumn{6}{|c|}{ Method Codes } \\
\hline & & & 0 & 3 & 4 & 6 & 10 & 11 \\
\hline 1 & 4 & -0.30 & - & 2.83 & - & - & -. & 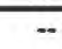 \\
\hline 5 & 3 & -0.65 & $\rightarrow$ & 2.7 & -. & - & .. & -. \\
\hline 7 & 4 & -0.11 & - & -. & -. & 2.9 & -. & .. \\
\hline 8 & 3 & -0.92 & .- & - & -. & - & -. & 2.6 \\
\hline 10 & 2 & -1.19 & -- & - & - & - & .. & 2.5 \\
\hline 12 & 0 & -2.54 & $\overline{-.}$ & 2 & $\cdots$ & 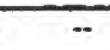 & -. & $\cdots$ \\
\hline 16 & 3 & -0.92 & -. & - & 2.6 & -. & -. & -. \\
\hline 23 & 0 & 38.20 & 17.1 & .. & -. & - & - & -. \\
\hline 25 & NR & -. & - & .. & $<16$ & - & -. & -. \\
\hline 32 & 4 & 0.43 & -- & -- & -. & 3.1 & - & - \\
\hline 42 & 4 & -0.24 & -- & $\cdots$ & -. & 2.85 & $\because$ & - \\
\hline 45 & 4 & 0.00 & -. & .. & -. & 2.94 & - & - \\
\hline 59 & NR & -- & -- & -. & -. & $<5$ & -- & - \\
\hline 70 & NR & -. & -. & - & -. & $<10$ & - & .. \\
\hline 76 & 4 & 0.13 & - & .- & .. & 2.987 & -- & - \\
\hline 89 & 0 & -2.54 & $\because$ & 2 & - & -- & -. & $\ldots$ \\
\hline 97 & 4 & -0.13 & -- & 2.89 & -- & - & -- & .. \\
\hline 105 & NR & -. & -- & -- & -. & $<7$ & -. & - \\
\hline 113 & 2 & -1.32 & - & 2.45 & .. & -- & - & -. \\
\hline 121 & 3 & 0.70 & - & -. & -- & 3.2 & -. & -. \\
\hline 134 & 4 & 0.03 & - & 2.95 & -- & - & 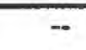 & -. \\
\hline 138 & 3 & 0.86 & -- & -- & -. & 3.26 & -. & - \\
\hline 142 & 4 & 0.32 & - & - & -. & 3.06 & -. & -. \\
\hline 146 & NR & - & - & -. & $<10.0$ & -- & -- & - \\
\hline 149 & 4 & 0.43 & -- & -. & - & 3.1 & -- & -. \\
\hline 180 & 4 & -0.40 & $\because$ & - & - & 2.79 & -- & - \\
\hline 183 & NR & . & .. & $<5.0$ & -. & -- & -- & -- \\
\hline 190 & 1 & -2.00 & - & 2.2 & -- & -- & -- & -. \\
\hline 193 & NR & - & - & $<5$ & -. & .. & - & .. \\
\hline 198 & 2 & 1.13 & - & -. & - & 3.36 & - & -- \\
\hline 212 & $\overline{N R}$ & -. & - & - & $<15$ & - & $\cdots$ & $\cdots$ \\
\hline 220 & 0 & -3.08 & -. & 1.8 & - & -- & $\ldots$ & -. \\
\hline 234 & 1 & -2.00 & - & 2.2 & -- & -- & - & -- \\
\hline 245 & 4 & 0.16 & -- & -. & -- & 3 & -- & -- \\
\hline 255 & 4 & 0.30 & $\cdots$ & -. & - & 3.05 & -- & - \\
\hline 256 & 0 & -6.42 & -- & -. & -. & .. & 0.56 & - \\
\hline 265 & 4 & 0.16 & - & -. & - & 3 & + & -- \\
\hline 305 & NR & -. & -- & .. & $<5$ & -. & -. & -. \\
\hline 328 & 4 & 0.43 & -. & - & - & 3.1 & .. & - \\
\hline 330 & 3 & 0.97 & - & -. & -- & 3.3 & $\cdots$ & -- \\
\hline
\end{tabular}


Table 11. Statistical summary of reported data for standard reference sample T-169 (trace constituents) -- continued T-169 SILICA (SiO2) in mg/L

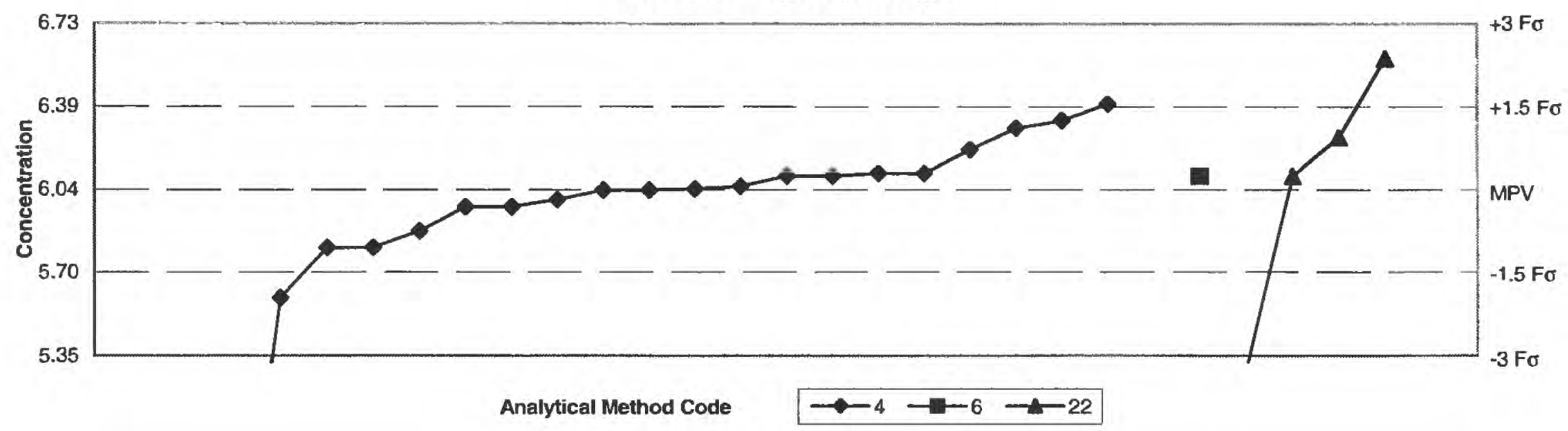

\begin{tabular}{|c|c|c|c|c|c|c|c|}
\hline \multirow[t]{2}{*}{ SUMMARY } & \multicolumn{5}{|c|}{ Methods } & \multicolumn{2}{|c|}{ Statlstics } \\
\hline & 4 & 5 & 6 & 22 & Method Codes & & \\
\hline $\mathrm{n}=$ & 22 & 1 & 1 & 4 & 04 Inductively coupled ptasma & MPV = & $6.04 \mathrm{mg} / \mathrm{L}$ \\
\hline Minimum $=$ & 2.9 & 2.46 & 6.1 & 5.3 & 05 Direct current plasma & F-pseudosigma $=$ & 0.23 \\
\hline Maximum $=$ & 6.4 & & & 6.59 & 06 Inductively coupled plasma/mass spectrometry & Rating criterion $=$ & 0.30 \\
\hline Median $=$ & 6.04 & & & & 22 Colorimetric & $n=$ & 28 \\
\hline F-pseudosigma $=$ & 0.23 & & & & & $\mathrm{Uh}=$ & 6.11 \\
\hline & & & & & & $\operatorname{Lh}=$ & 5.80 \\
\hline
\end{tabular}

\begin{tabular}{|c|c|c|c|c|c|c|}
\hline \multirow[b]{2}{*}{ Lab } & \multirow[b]{2}{*}{ Rating } & \multirow[b]{2}{*}{$Z$-value } & \multicolumn{4}{|c|}{ Method Codes } \\
\hline & & & 4 & 5 & 6 & 22 \\
\hline 1 & 4 & 0.19 & 6.1 & $\cdots$ & -- & - \\
\hline 5 & 0 & -10.40 & 2.9 & - & -- & - \\
\hline 7 & 3 & -0.57 & 5.87 & $\cdots$ & - & -- \\
\hline 8 & 2 & $t .18$ & 6.4 & - & -- & -- \\
\hline 24 & 3 & 0.55 & 6.21 & $\ldots$ & -- & - \\
\hline 25 & 0 & -6.17 & 4.18 & 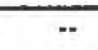 & -- & - \\
\hline 32 & 4 & 0.19 & -- & - & 6.1 & - \\
\hline 33 & 0 & -11.86 & -- & 2.46 & -- & -. \\
\hline 42 & 2 & -1.50 & 5.59 & - & - & - \\
\hline 64 & 3 & -0.80 & 5.8 & - & - & - \\
\hline 70 & 3 & 0.72 & - & - & 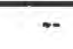 & 6.26 \\
\hline 97 & 1 & 1.81 & - & - & - & 6.59 \\
\hline 105 & 4 & 0.04 & 6.056 & - & -- & - \\
\hline 110 & 4 & 0.01 & 6.046 & - & - & - \\
\hline 121 & 4 & -0.14 & 6 & .. & - & - \\
\hline 134 & 4 & -0.01 & 6.04 & 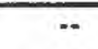 & - & $\rightarrow$ \\
\hline 142 & 4 & -0.01 & 6.04 & - & - & -- \\
\hline 190 & 4 & 0.19 & -. & - & -- & 6.1 \\
\hline 212 & 4 & 0.19 & 6.1 & - & - & -- \\
\hline 220 & 4 & 0.22 & 6.11 & - & - & -. \\
\hline 234 & 4 & -0.24 & 5.97 & - & - & -- \\
\hline 256 & 0 & -2.46 & -. & - & -. & 5.3 \\
\hline 259 & 4 & -0.24 & 5.97 & - & - & -- \\
\hline 265 & 3 & -0.80 & 5.8 & .. & - & -. \\
\hline 328 & 3 & 0.85 & 6.3 & -. & - & $\ldots$ \\
\hline 356 & 3 & 0.95 & 6.33 & $=$ & $=$ & $\cdots$ \\
\hline 370 & 0 & -10.07 & 3 & -. & - & - \\
\hline 372 & 4 & 0.22 & 6.11 & -. & - & -- \\
\hline
\end{tabular}


Table 11. Statistical summary of reported data for standard reference sample T-169 (trace constituents) -- continued

T-169 STRONTIUM (Sr) in $\mu g / L$

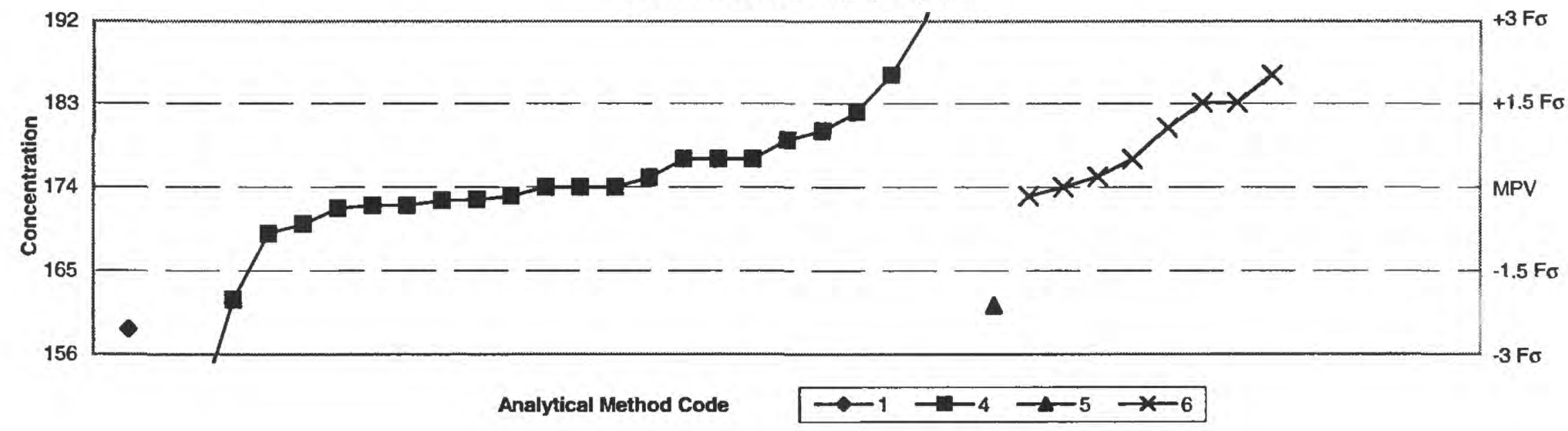

\begin{tabular}{|c|c|c|c|c|c|c|c|}
\hline \multirow[t]{2}{*}{ SUMMARY } & \multicolumn{5}{|c|}{ Methods } & \multicolumn{2}{|c|}{ Statistics } \\
\hline & 1 & 4 & 5 & 6 & Method Codes & & \\
\hline $\mathbf{n}=$ & 1 & 24 & 1 & 8 & 01 Atomic absorption: direct, air & MPV $=$ & $174 \mu \mathrm{g} / \mathrm{L}$ \\
\hline Minimum = & 158.9 & 150 & 161.4 & 173 & 04 inductively coupled ptasma & F-pseudosigma $=$ & 6 \\
\hline Maximum $=$ & & 203 & & 186 & 05 Direct currenl plasma & Rating criterion = & 9 \\
\hline Median $=$ & & 174 & & 179 & 06 Inductively coupled plasma/mass spectrometry & $n=$ & 34 \\
\hline F-pseudosigma = & & 5 & & 6 & & $U_{h}=$ & 180 \\
\hline & & & & & & $\mathrm{Lh}=$ & 172 \\
\hline
\end{tabular}

\begin{tabular}{|c|c|c|c|c|c|c|}
\hline \multirow[b]{2}{*}{ Lab } & \multirow[b]{2}{*}{ Rating } & \multirow[b]{2}{*}{$Z$-value } & \multicolumn{4}{|r|}{ Method Codes } \\
\hline & & & 1 & 4 & 5 & 6 \\
\hline 1 & 4 & -0.26 & - & 171.7 & $\overline{--}$ & -- \\
\hline 5 & 4 & 0.00 & - & 174 & -- & - \\
\hline 7 & 4 & 0.11 & - & 175 & - & -. \\
\hline 8 & 2 & 1.38 & - & -- & - & 186 \\
\hline 16 & 3 & -0.57 & -- & 169 & .. & .. \\
\hline 24 & 4 & 0.34 & $\overline{-}$ & 177 & $\overline{--}$ & -- \\
\hline 25 & 4 & -0.11 & - & 173 & -- & -- \\
\hline 32 & 2 & 1.03 & - & -- & -- & 183 \\
\hline 33 & 2 & -1.45 & -- & -- & 161.4 & - \\
\hline 42 & 0 & 2.07 & - & 192 & - & -. \\
\hline 59 & 4 & -0.11 & - & - & - & 173 \\
\hline 76 & 4 & 0.13 & - & -- & - & 175.1 \\
\hline 86 & 4 & 0.00 & - & 174 & - & -- \\
\hline 97 & 4 & -0.46 & -- & 170 & -. & -- \\
\hline 105 & 4 & 0.00 & -. & 174 & - & -. \\
\hline 109 & 1 & -1.74 & 158.9 & -- & - & - \\
\hline 113 & 4 & -0.16 & - & 172.6 & - & -- \\
\hline 121 & 4 & 0.34 & - & 177 & -- & -- \\
\hline 134 & 4 & -0.17 & - & 172.6 & -- & -- \\
\hline 138 & 4 & -0.23 & - & 172 & - & -. \\
\hline 142 & 3 & 0.57 & - & 179 & - & - \\
\hline 147 & 4 & 0.00 & - & -. & -- & 174 \\
\hline 212 & 3 & 0.92 & -- & 182 & - & -. \\
\hline 234 & 4 & 0.34 & - & 177 & - & -- \\
\hline 245 & 3 & 0.72 & - & -- & - & 180.3 \\
\hline 247 & 0 & -2.76 & - & 150 & $\ldots$ & -- \\
\hline 256 & 2 & 1.38 & -. & 186 & -- & -- \\
\hline 259 & 3 & 0.69 & -- & 180 & - & - \\
\hline 265 & 4 & -0.23 & -- & 172 & - & -- \\
\hline 304 & 4 & 0.34 & - & -- & - & 177 \\
\hline 328 & 0 & -2.76 & - & 150 & -- & - \\
\hline 330 & 2 & 1.03 & - & -- & - & 183 \\
\hline 356 & 0 & 3.33 & - & 203 & - & -- \\
\hline 372 & 2 & -1.38 & - & 162 & - & -- \\
\hline
\end{tabular}


Table 11. Statistical summary of reported data for standard reference sample T-169 (trace constituents) -- continued

T-169 THALLIUM (TI) in $\mu \mathrm{g} / \mathrm{L}$

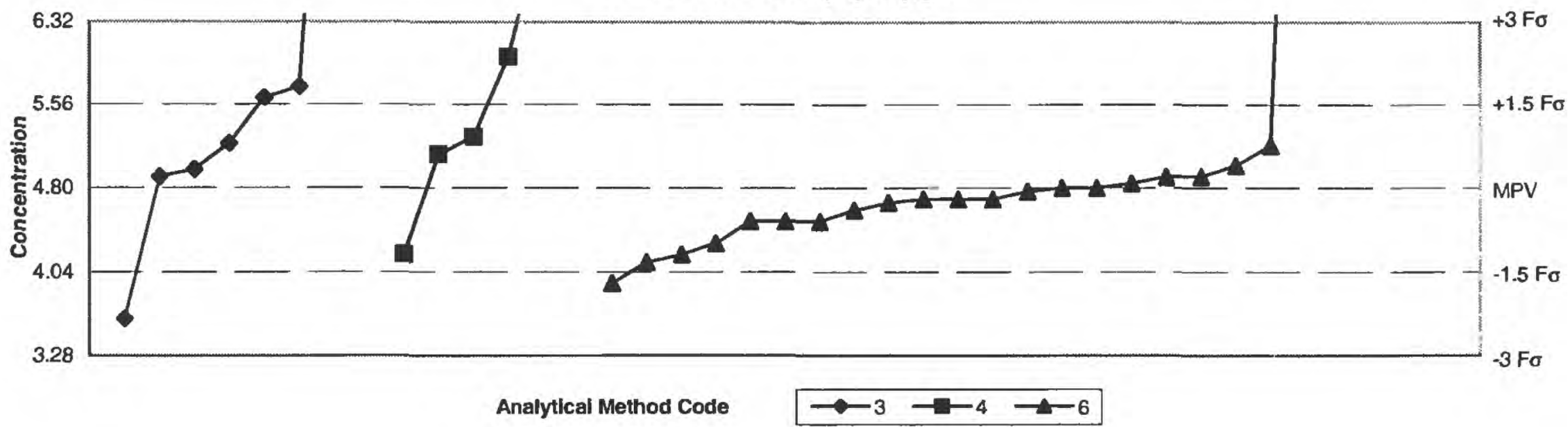

\begin{tabular}{|c|c|c|c|c|c|c|}
\hline \multirow[t]{2}{*}{ SUMMARY } & \multicolumn{4}{|c|}{ Methods } & \multicolumn{2}{|c|}{ Statistics } \\
\hline & 3 & 4 & 6 & Method Codes & & \\
\hline $\mathrm{n}=$ & 7 & 5 & 21 & 03 Atomic absorption: graphite fumace & MPV $=$ & $4.80 \mu \mathrm{g} / \mathrm{L}$ \\
\hline Minimurn $=$ & 3.62 & 4.2 & 3.94 & 04 Inductively coupled plasma & F-pseudosigma $=$ & 0.51 \\
\hline Maximum $=$ & 10.3 & 7.26 & 12.8 & 06 inductively coupled plasma/mass spectrometry & $\mathrm{n}=$ & 33 \\
\hline Median $=$ & 5.20 & 5.26 & 4.70 & & $\mathrm{Uh}=$ & 5.18 \\
\hline F-pseudosigma $=$ & 0.55 & 0.67 & 0.25 & & $\operatorname{Ln}=$ & 4.50 \\
\hline
\end{tabular}

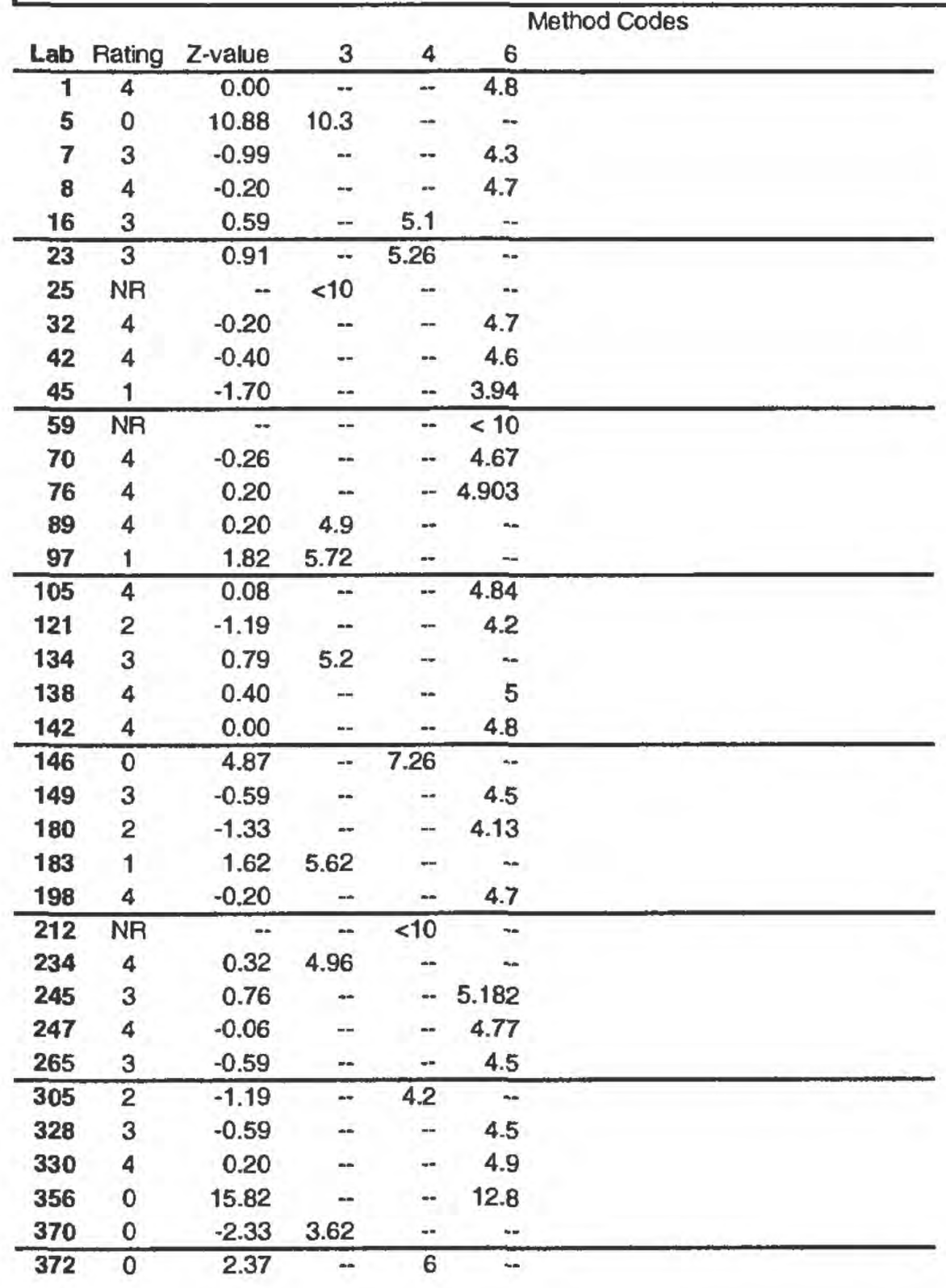


Table 11. Statistical summany of reported data for standard reference sample T-169 (trace constituents) -- continued

T-169 URANIUM (U) in $\mu \mathrm{g} / \mathrm{L}$

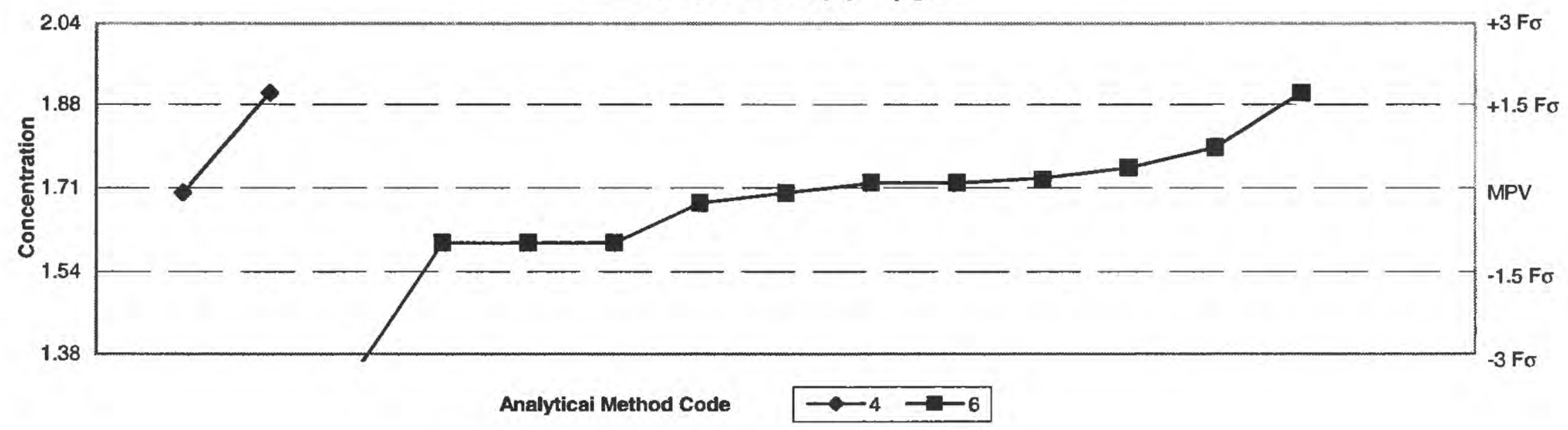

\begin{tabular}{|c|c|c|c|c|}
\hline \multirow[t]{2}{*}{ SUMMARY } & \multicolumn{2}{|c|}{ Methods } & \multicolumn{2}{|c|}{ Statistics } \\
\hline & 4 & Method Codes & & \\
\hline $\mathrm{n}=$ & 12 & 04 Inductively coupled plasma & MPV $=$ & $1.71 \mu \mathrm{g} / \mathrm{L}$ \\
\hline Minimum $=$ & 1.34 & 06 Inductively coupled plasma/mass spectrometry & F-pseudosigma $=$ & 0.11 \\
\hline Maximum $=$ & 1.9 & & $\mathrm{n}=$ & 14 \\
\hline Median $=$ & 1.71 & & $\mathrm{Uh}=$ & 1.75 \\
\hline F-pseudosigma $=$ & 0.10 & & $\operatorname{Lh}=$ & 1.60 \\
\hline
\end{tabular}

\begin{tabular}{rrrrrr}
\hline & & & & & Method Codes \\
\hline \multicolumn{1}{l}{ Lab } & Rating & Z-vaiue & 4 & 6 & \\
\hline 1 & 4 & 0.18 & - & 1.73 & \\
7 & 3 & -0.99 & - & 1.6 & \\
8 & 3 & -0.99 & - & 1.6 & \\
16 & 1 & 1.71 & 1.9 & - & \\
32 & 3 & 0.72 & - & 1.79 & \\
\hline 42 & 4 & 0.36 & - & 1.75 & \\
$\mathbf{4 5}$ & 0 & -3.33 & - & 1.34 \\
70 & NR & - & - & $<10.0$ \\
142 & 4 & 0.09 & - & 1.72 \\
147 & 4 & -0.27 & - & 1.68 \\
\hline 149 & 3 & -0.99 & - & 1.6 \\
245 & 4 & 0.09 & - & 1.72 \\
265 & 4 & -0.09 & - & 1.7 \\
328 & 4 & -0.09 & 1.7 & - \\
330 & 1 & 1.71 & - & 1.9 \\
\hline
\end{tabular}


Table 11. Statistical summary of reported data for standard reference sample T-169 (trace constituents) -- continued

T-169 VANADIUM (V) in $\mu \mathrm{g} / \mathrm{L}$

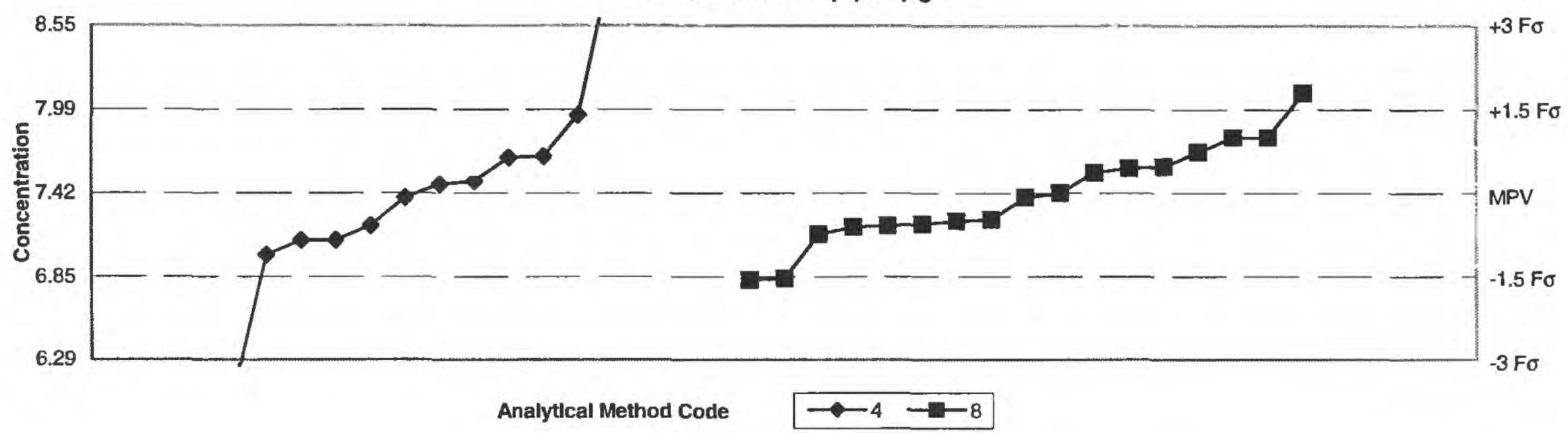

\begin{tabular}{|c|c|c|c|c|c|c|}
\hline \multirow[t]{3}{*}{ SUMMARY } & \multicolumn{4}{|c|}{ Methods } & \multicolumn{2}{|c|}{ Statistics } \\
\hline & 3 & 4 & 6 & Method Codes & & \\
\hline & 2 & 14 & 17 & 03 Atomic absorption: graphite fumace & MPV $=$ & $7.42 \mu \mathrm{g} / \mathrm{L}$ \\
\hline Minimum $=$ & 10.09 & 1.5 & 6.83 & 04 Inductively coupled plasma & F-pseudosigma $=$ & 0.38 \\
\hline Maximum $=$ & 10.2 & 19 & 8.1 & 06 Inductively coupled plasma/mass spectrometry & $n=$ & 33 \\
\hline Median = & & 7.44 & 7.39 & & $U \mathrm{~h}=$ & 7.70 \\
\hline F-pseudosigma $=$ & & 0.42 & 0.30 & & $\mathrm{Lh}=$ & 7.t9 \\
\hline
\end{tabular}

\begin{tabular}{|c|c|c|c|c|c|c|}
\hline & & & & & & Method Codes \\
\hline Lab & Rating & Z-value & 3 & 4 & 6 & \\
\hline 1 & 4 & 0.45 & -- & -- & 7.59 & \\
\hline 5 & 3 & -0.85 & -- & 7.1 & .. & \\
\hline 7 & 3 & 1.01 & -. & - & 7.8 & \\
\hline 8 & 4 & 0.48 & -. & -- & 7.6 & \\
\hline 16 & 2 & -1.11 & -- & 7 & -- & \\
\hline 25 & NR & - & -- & $<19$ & - & \\
\hline 32 & 3 & 0.74 & -- & -- & 7.7 & \\
\hline 42 & 4 & -0.48 & -- & -- & 7.24 & \\
\hline 45 & 3 & -0.74 & -. & -- & 7.14 & \\
\hline 59 & 3 & -0.56 & -. & .. & 7.21 & \\
\hline 70 & 4 & 0.37 & 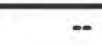 & -- & 7.56 & \\
\hline 86 & 3 & -0.58 & -- & 7.2 & -- & \\
\hline 89 & 0 & 7.35 & 10.2 & - & - & \\
\hline 97 & NR & -. & - & $<7.7$ & - & \\
\hline 105 & NR & -- & -. & $\cdots$ & $<20$ & \\
\hline 121 & 3 & 1.01 & - & 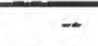 & 7.8 & \\
\hline 134 & 4 & 0.16 & - & 7.48 & -- & \\
\hline 138 & 4 & -0.08 & -. & 7.39 & -. & \\
\hline 142 & 4 & 0.00 & - & -- & 7.42 & \\
\hline 146 & 2 & 1.40 & - & 7.95 & - & \\
\hline 149 & 3 & -0.58 & - & - & 7.2 & \\
\hline 180 & 1 & -1.53 & - & -- & 6.84 & \\
\hline 183 & 0 & 7.06 & 10.09 & -- & -- & \\
\hline 198 & 4 & -0.50 & - & -- & 7.23 & \\
\hline 212 & 0 & 4.18 & - & 9 & -- & \\
\hline 234 & 3 & 0.66 & -- & 7.67 & $=$ & \\
\hline 245 & 4 & -0.08 & - & -- & 7.39 & \\
\hline 247 & 3 & -0.61 & .. & -- & 7.19 & \\
\hline 256 & 3 & 0.63 & -- & 7.66 & -- & \\
\hline 265 & 4 & 0.21 & -- & 7.5 & .. & \\
\hline 305 & 3 & -0.85 & -- & 7.1 & -- & \\
\hline 328 & 0 & -15.66 & -- & 1.5 & -. & \\
\hline 330 & 1 & 1.80 & -. & - & 8.1 & \\
\hline 356 & 1 & -1.56 & -- & -- & 6.83 & \\
\hline 370 & 0 & 30.63 & - & 19 & -. & \\
\hline 372 & 0 & -3.76 & -- & 6 & - & \\
\hline
\end{tabular}


Table 11. Statistical summary of reported data for standard reference sample T-169 (trace constituents) -- continued

T-169 ZINC (Zn) in $\mu \mathrm{g} / \mathrm{L}$

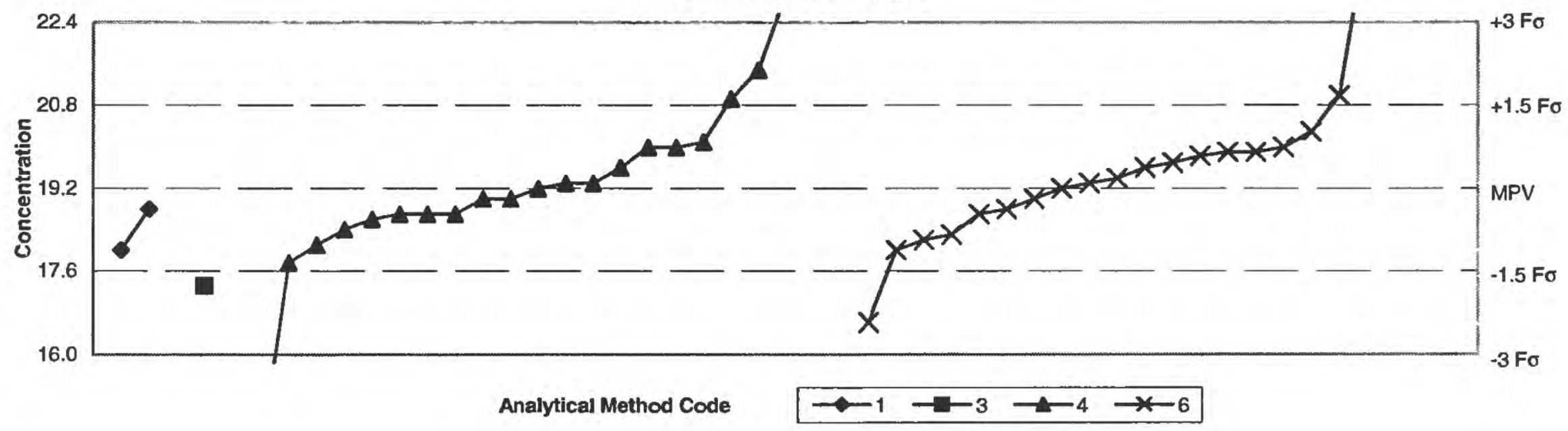

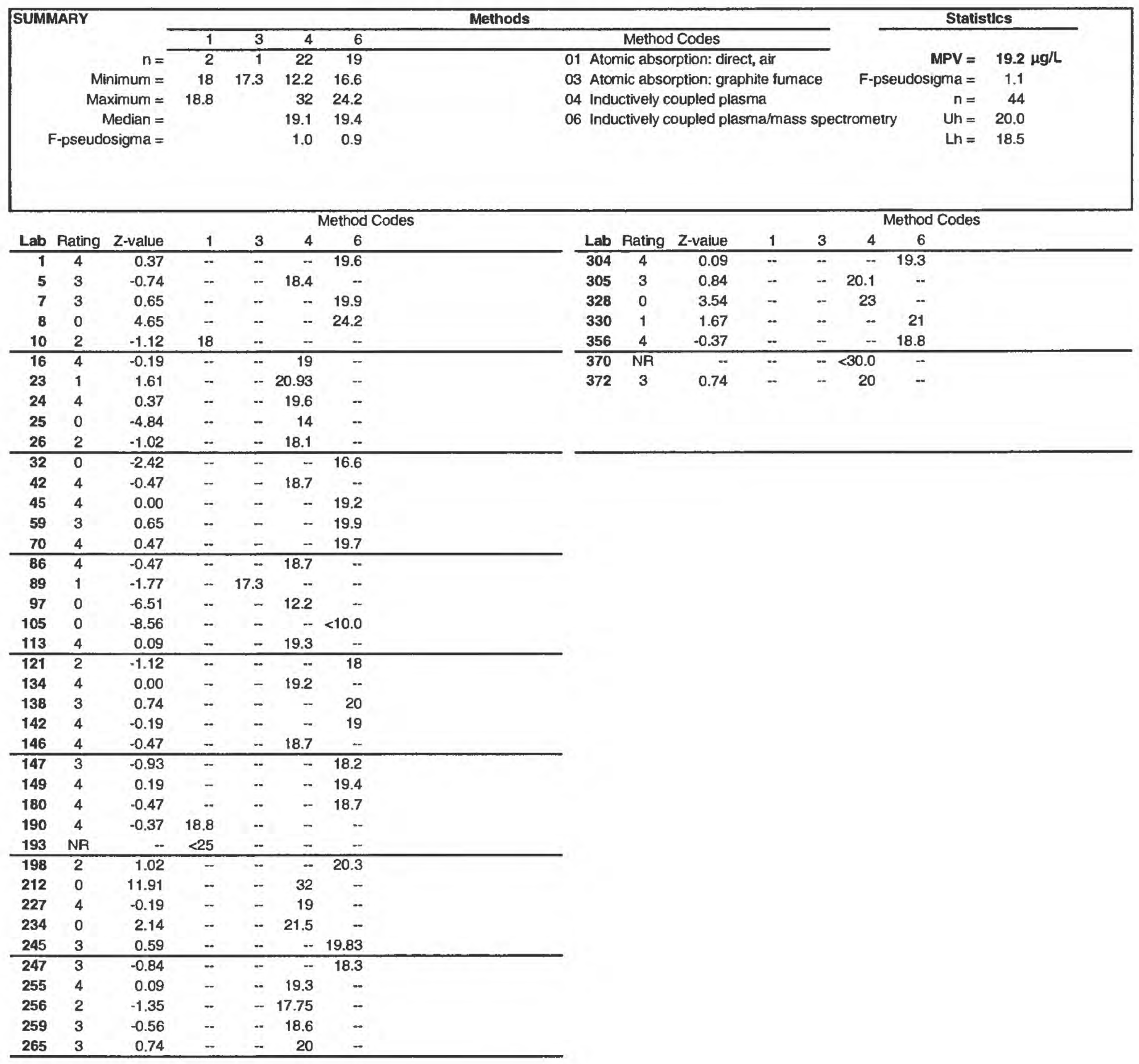


Table 12. Statistical summary of reported data for standard reference sample M-162 (major constituents)

M-162 ALKALINITY (as $\mathrm{CaCO}_{3}$ ) in mg/L

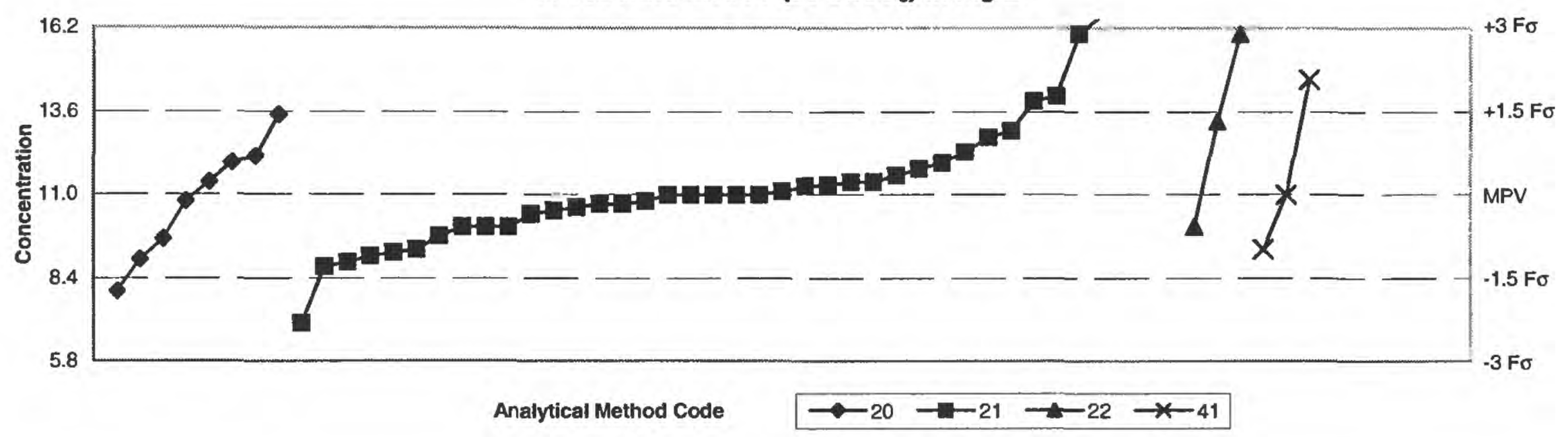

\begin{tabular}{|c|c|c|c|c|c|c|c|}
\hline \multirow[t]{2}{*}{ SUMMARY } & \multicolumn{5}{|c|}{ Methods } & \multicolumn{2}{|c|}{ Statistics } \\
\hline & 20 & 21 & 22 & 41 & Method Codes & & \\
\hline$n=$ & 8 & 39 & 3 & 3 & 20 Titration: colorimetric & $M P V=$ & $11.0 \mathrm{mg} / \mathrm{L}$ \\
\hline Minimum $=$ & 8 & 7 & 10 & 9.3 & 21 Titration: electrometric & F-pseudosigma = & 1.7 \\
\hline Maximum $=$ & 13.5 & 22 & 16 & 14.6 & 22 Colorimetric & $\mathrm{n}=$ & 53 \\
\hline Median = & 11.1 & 11.0 & & & 41 Electrometric & $\mathrm{Uh}=$ & 12.3 \\
\hline F-pseudosigma $=$ & 2.1 & 1.5 & & & & $\operatorname{Lh}=$ & 10.0 \\
\hline
\end{tabular}

\begin{tabular}{|c|c|c|c|c|c|c|c|c|c|c|c|c|c|}
\hline \multirow[b]{2}{*}{ Lab } & \multicolumn{6}{|c|}{ Method Codes } & \multirow[b]{2}{*}{ Lab } & \multirow[b]{2}{*}{ Rating } & \multirow[b]{2}{*}{$Z$-value } & \multicolumn{4}{|c|}{ Method Codes } \\
\hline & Rating & Z-value & 20 & 21 & 22 & 41 & & & & 20 & 21 & 22 & 41 \\
\hline 1 & 4 & 0.16 & - & 11.28 & $\cdots$ & -- & 259 & 4 & -0.29 & - & 10.5 & - & -- \\
\hline 4 & 0 & 6.36 & - & 22 & -- & - & 263 & 4 & 0.23 & - & 11.4 & -- & -- \\
\hline 5 & 4 & -0.17 & - & 10.7 & - & - & 266 & 4 & 0.06 & -- & 11.1 & -- & -- \\
\hline 8 & 2 & 1.16 & -- & 13 & -- & -- & 269 & 4 & 0.00 & -. & 11 & -- & - \\
\hline 10 & 4 & -0.17 & - & 10.7 & -- & -- & 321 & 0 & -2.31 & - & 7 & -. & - \\
\hline 12 & 4 & 0.00 & - & 11 & - & - & 328 & 1 & -1.73 & 8 & - & $-\cdot$ & -- \\
\hline 16 & 3 & -0.58 & -. & -- & 10 & -- & $\mathbf{3 3 0}$ & 0 & 5.20 & -- & 20 & -- & -- \\
\hline 23 & 3 & 0.58 & 12 & - & -- & - & 333 & 3 & -0.98 & -. & 9.3 & -. & -- \\
\hline 24 & 4 & 0.00 & -. & 11 & -- & -- & 341 & 0 & 2.89 & - & -. & 16 & -- \\
\hline 25 & 0 & 2.89 & - & 16 & -- & - & 356 & 4 & 0.17 & -- & 11.3 & .. & -- \\
\hline 32 & 4 & -0.12 & 10.8 & - & -- & - & 366 & 3 & -0.79 & 9.64 & -- & -- & - \\
\hline 33 & 2 & -1.10 & -- & 9.1 & - & - & 370 & 3 & -0.58 & . & 10 & -. & -- \\
\hline 38 & 4 & -0.23 & - & 10.61 & - & -- & 372 & 0 & 5.43 & -. & 20.4 & -. & -- \\
\hline 42 & 1 & 1.79 & -. & 14.1 & - & - & & & & & & & \\
\hline 45 & 4 & 0.23 & - & 11.4 & - & - & & & & & & & \\
\hline 46 & 4 & 0.23 & 11.4 & - & -- & - & & & & & & & \\
\hline 59 & 2 & -1.29 & -- & 8.77 & -- & - & & & & & & & \\
\hline 70 & 4 & 0.00 & -. & -. & -- & 11 & & & & & & & \\
\hline 85 & 2 & 1.04 & -. & 12.8 & -- & -- & & & & & & & \\
\hline 89 & 4 & -0.35 & - & 10.4 & - & - & & & & & & & \\
\hline 97 & 3 & -0.58 & - & 10 & - & - & & & & & & & \\
\hline 105 & 4 & 0.46 & -- & 11.8 & -- & - & & & & & & & \\
\hline 109 & 1 & 1.70 & - & 13.94 & -- & -- & & & & & & & \\
\hline 113 & 3 & -0.74 & - & 9.72 & - & -. & & & & & & & \\
\hline 134 & 3 & 0.58 & - & 12 & - & - & & & & & & & \\
\hline 138 & 2 & -1.03 & - & 9.21 & - & 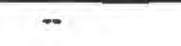 & & & & & & & \\
\hline 142 & 4 & 0.35 & -. & 11.6 & -- & - & & & & & & & \\
\hline 146 & 0 & 3.24 & - & 16.6 & -- & - & & & & & & & \\
\hline 149 & 4 & 0.00 & -. & 11 & - & .. & & & & & & & \\
\hline 180 & 0 & 2.08 & -- & -- & - & 14.6 & & & & & & & \\
\hline 183 & 2 & -1.16 & 9 & - & - & - & & & & & & & \\
\hline 190 & 4 & -0.12 & - & 10.8 & -. & -. & & & & & & & \\
\hline 193 & 3 & -0.98 & -. & -. & -- & 9.3 & & & & & & & \\
\hline 212 & 2 & -1.21 & -- & 8.9 & - & -. & & & & & & & \\
\hline 220 & 3 & 0.77 & - & 12.33 & - & - & & & & & & & \\
\hline 224 & 3 & -0.58 & - & 10 & - & 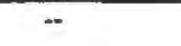 & & & & & & & \\
\hline 227 & 2 & 1.33 & -. & -. & 13.3 & - & & & & & & & \\
\hline 234 & 3 & 0.69 & 12.2 & -. & - & -. & & & & & & & \\
\hline 247 & 4 & 0.00 & -- & 11 & - & -- & & & & & & & \\
\hline 256 & 2 & 1.44 & 13.5 & - & - & 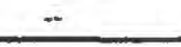 & & & & & & & \\
\hline
\end{tabular}




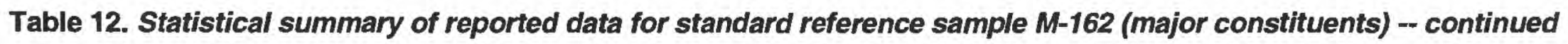

M-162 BORON (B) in $\mu g / L$

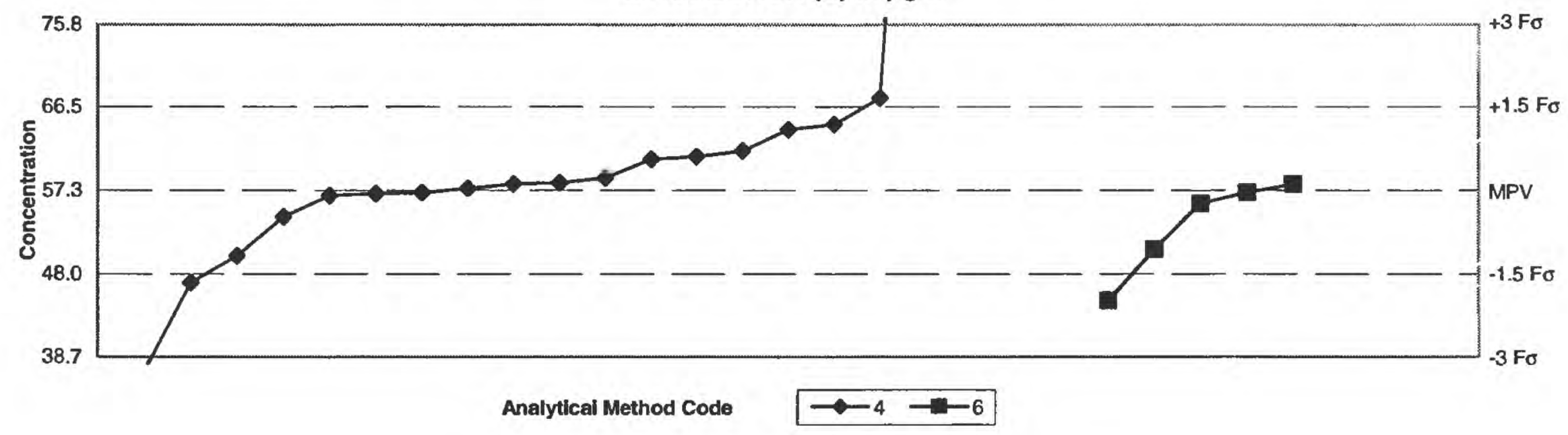

\begin{tabular}{|c|c|c|c|c|c|c|}
\hline \multirow[t]{2}{*}{ SUMMARY } & \multicolumn{4}{|r|}{ Mothods } & \multicolumn{2}{|c|}{ Statistics } \\
\hline & 4 & 6 & 22 & Method Codes & & \\
\hline$n=$ & 18 & 5 & 1 & 04 Inductively coupled plasma & MPV $=$ & $57.3 \mu \mathrm{g} / \mathrm{L}$ \\
\hline Minimum $=$ & 37 & 45 & 0.21 & 06 Inductively coupled plasma/mass spectromeiry & F-pseudosigma $=$ & 6.2 \\
\hline Maximum $=$ & 140 & 58 & & 22 Colorimetric & $n=$ & 24 \\
\hline Median $=$ & 58.1 & 55.8 & & & $\mathrm{Uh}=$ & 60.9 \\
\hline F-pseudosigma $=$ & 3.6 & 4.7 & & & $\mathrm{Lh}=$ & 52.5 \\
\hline
\end{tabular}

\begin{tabular}{|c|c|c|c|c|c|c|}
\hline \multirow[b]{2}{*}{ Lab } & \multirow[b]{2}{*}{ Rating } & \multirow[b]{2}{*}{$Z$-value } & \multirow{2}{*}{\multicolumn{4}{|c|}{ Method Codes }} \\
\hline & & & 4 & & & \\
\hline 5 & 0 & -7.63 & $<10.0$ & - & 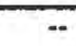 & \\
\hline 8 & 1 & -1.98 & -- & 45 & .. & \\
\hline 16 & 1 & -1.66 & 47 & .. & -. & \\
\hline 23 & 0 & -9.22 & .. & .- & 0.21 & \\
\hline 24 & 4 & -0.09 & 56.7 & -- & -. & \\
\hline 25 & 2 & -1.17 & 50 & -. & 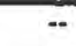 & \\
\hline 32 & 4 & -0.04 & -. & 57 & -- & \\
\hline 42 & 4 & -0.48 & 54.3 & -. & .. & \\
\hline 45 & 4 & -0.23 & .. & 55.8 & - & \\
\hline 59 & 2 & -1.06 & .. & 50.7 & -- & \\
\hline 70 & NR & -- & $<100$ & .. & .. & \\
\hline 85 & 0 & -3.27 & 37 & -- & -- & \\
\hline 86 & 4 & 0.14 & 58.1 & -- & -. & \\
\hline 105 & NR & -. & $<200$ & -- & -- & \\
\hline 134 & 4 & 0.22 & 58.63 & - & -- & \\
\hline 138 & 4 & 0.04 & 57.5 & $=$ & -. & \\
\hline 142 & 3 & 0.56 & 60.7 & -. & .. & \\
\hline 180 & 1 & 1.66 & 67.5 & .- & .. & \\
\hline 212 & 4 & -0.06 & 56.9 & -. & -. & \\
\hline 234 & 2 & 1.09 & 64 & .- & .. & \\
\hline 247 & 0 & 13.37 & 140 & - & 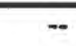 & \\
\hline 259 & 4 & 0.12 & 58 & -. & -. & \\
\hline 265 & 4 & 0.12 & -- & 58 & -- & \\
\hline 319 & 3 & 0.70 & 61.6 & .. & .. & \\
\hline 328 & 3 & 0.61 & 61 & -. & -. & \\
\hline 341 & 2 & 1.19 & 64.6 & - & - & \\
\hline 372 & 4 & -0.04 & 57 & .- & .. & \\
\hline
\end{tabular}


M-162 CALCIUM (Ca) in mg/L

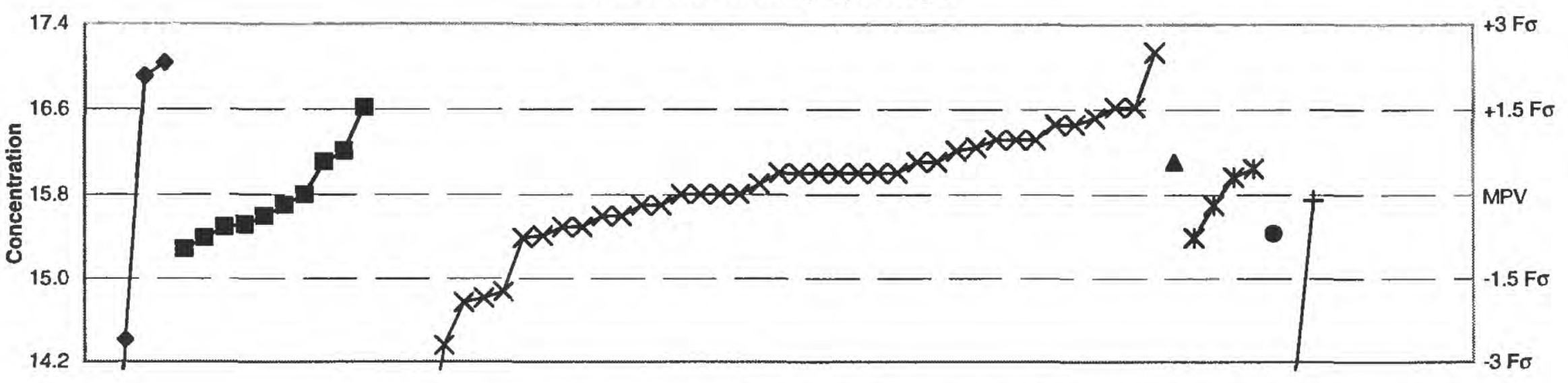

Analytical Method Code

$\rightarrow-0-1 \rightarrow-1 \rightarrow-5 \rightarrow-6 \rightarrow-7 \rightarrow-20$

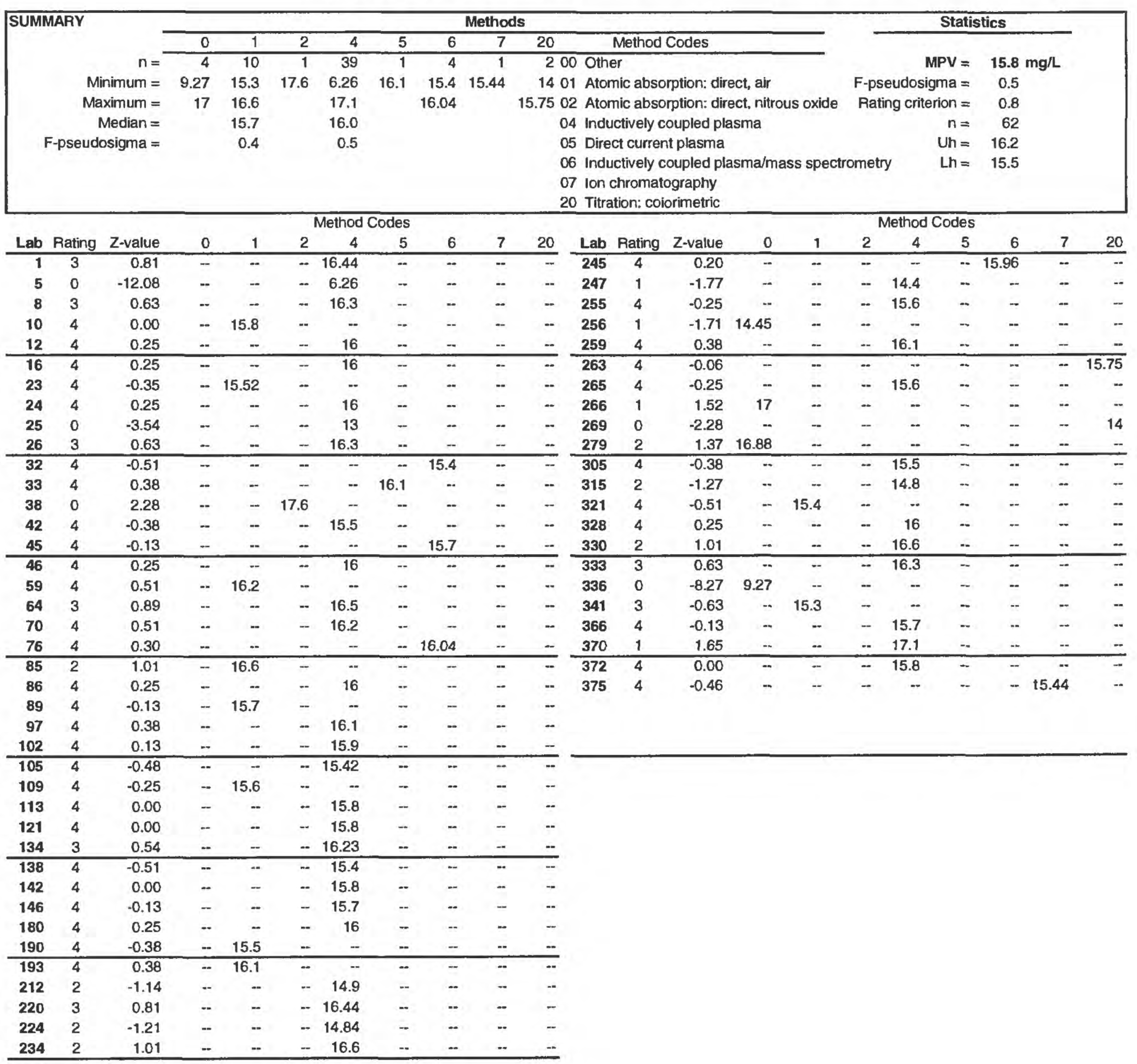


M-162 CHLORIDE (Cl) In mg/L

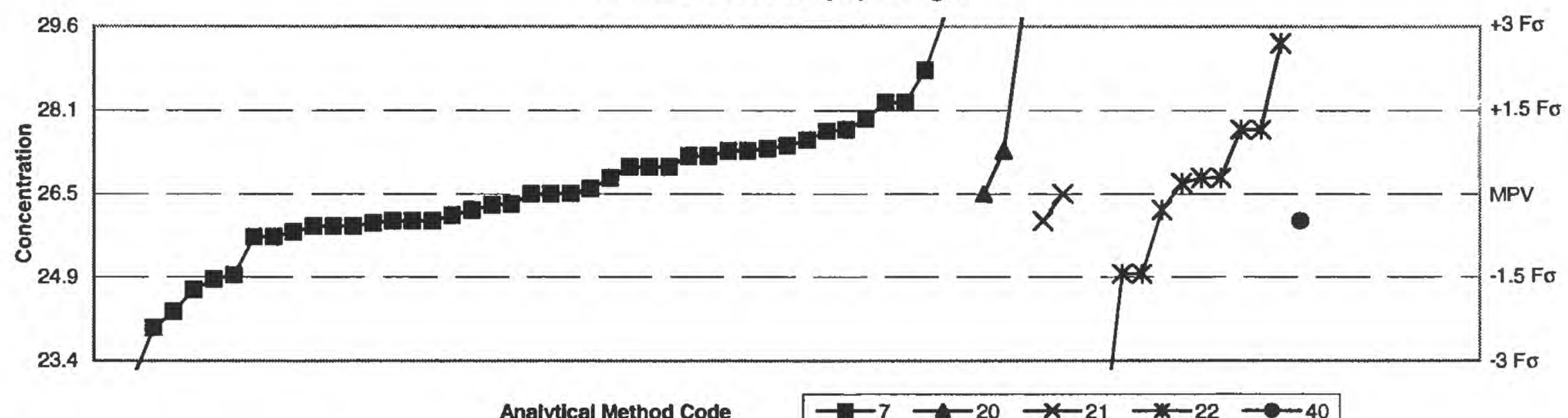

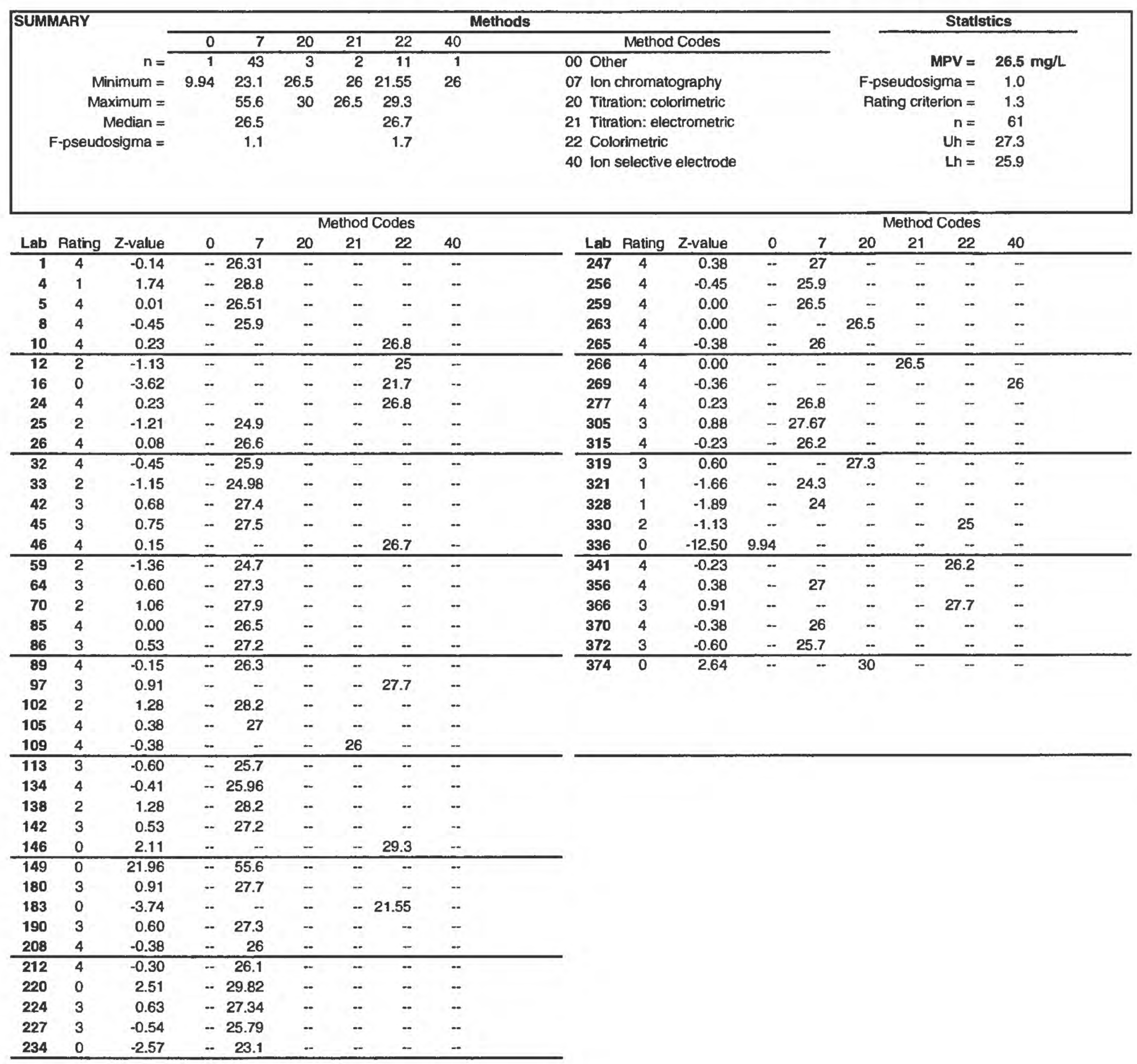


Table 12. Statistical summary of reported data for standard reference sample M-162 (major constituents) -- continued

M-162 FLUORIDE (F) in mg/L

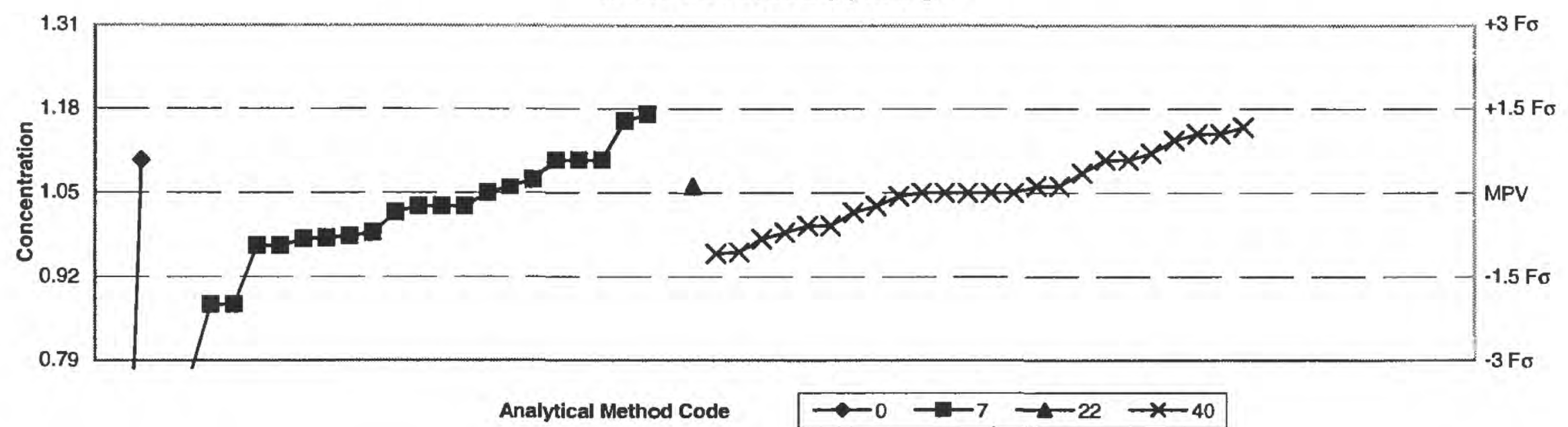

\begin{tabular}{|c|c|c|c|c|c|c|c|}
\hline \multirow[t]{2}{*}{ SUMMARY } & \multicolumn{5}{|c|}{ Methods } & \multicolumn{2}{|c|}{ Statlstics } \\
\hline & 0 & 7 & 22 & 40 & Method Codes & & \\
\hline$n=$ & 2 & 22 & 1 & 24 & 00 Other & MPV $=$ & $1.05 \mathrm{mg} / \mathrm{L}$ \\
\hline Minimum $=$ & 0.13 & 0.04 & 1.06 & 0.958 & 07 ion chromatography & F-pseudosigma $=$ & 0.09 \\
\hline Maximum = & 1.1 & 1.17 & & 1.15 & 22 Colorimetric & $n=$ & 49 \\
\hline Median $=$ & & 1.03 & & 1.05 & 40 Ion selective electrode & Uh $=$ & 1.10 \\
\hline F-pseudosigma = & & 0.07 & & 0.07 & & $\operatorname{Lh}=$ & 0.99 \\
\hline
\end{tabular}

\begin{tabular}{|c|c|c|c|c|c|c|c|c|c|c|c|c|c|}
\hline \multicolumn{10}{|c|}{ Method Codes } & \multicolumn{4}{|c|}{ Method Codes } \\
\hline Lab & Rating & Z-value & 0 & 7 & 22 & 40 & Lab & Rating & Z-value & 0 & 7 & 22 & 40 \\
\hline 1 & 4 & 0.12 & - & - & -- & 1.06 & 266 & 3 & -0.59 & 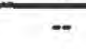 & -- & - & 1 \\
\hline 5 & 3 & -0.70 & - & 0.99 & - & - & 269 & 2 & -1.06 & -- & -. & - & 0.96 \\
\hline 8 & 1 & -1.99 & - & 0.88 & -- & - & 277 & 3 & -0.82 & - & 0.98 & -- & - \\
\hline 10 & 2 & 1.06 & - & -- & - & 1.14 & 305 & 2 & -1.08 & - & -- & - & 0.958 \\
\hline 16 & 4 & -0.23 & - & 1.03 & - & - & 328 & 3 & -0.82 & - & - & - & 0.98 \\
\hline 23 & 4 & 0.35 & 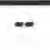 & - & $\because$ & 1.08 & 330 & 2 & 1.06 & - & - & $\cdots$ & 1.14 \\
\hline 24 & 4 & 0.12 & -- & - & - & 1.06 & 336 & 0 & -10.79 & 0.13 & - & - & -- \\
\hline 25 & 0 & -11.85 & - & 0.04 & - & -- & 356 & 3 & -0.76 & - & 0.985 & -- & -- \\
\hline 26 & 1 & -1.99 & -- & 0.88 & -- & -- & 370 & 4 & -0.35 & .. & 1.02 & -- & - \\
\hline 32 & 4 & -0.23 & - & 1.03 & -. & - & 372 & 0 & -3.40 & -. & 0.76 & - & - \\
\hline
\end{tabular}


Table 12. Statistical summary of reported data for standard reference sample M-162 (major constituents) -- continued M-162 POTASSIUM (K) in mg/L

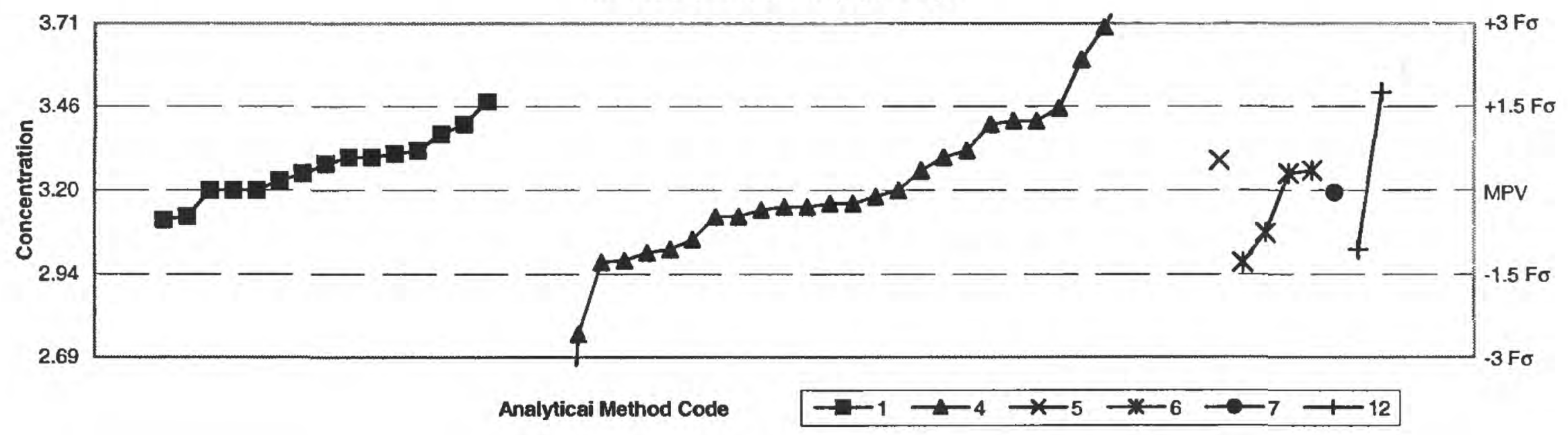

\begin{tabular}{|c|c|c|c|c|c|c|c|c|c|c|}
\hline \multirow[t]{2}{*}{ SUMMARY } & \multicolumn{8}{|c|}{ Methods } & \multicolumn{2}{|c|}{ Statistics } \\
\hline & 0 & 1 & 4 & 5 & 6 & 7 & 12 & Method Codes & & \\
\hline$n=$ & 2 & 15 & 30 & 1 & 4 & 1 & 2 & OO Other & MPV $=$ & $3.20 \mathrm{mg} / \mathrm{L}$ \\
\hline Minimum = & 0.79 & 3.11 & 0.99 & 3.29 & 2.98 & 3.193 & 3.02 & 01 Atomic absorption: direct, air & losigma $=$ & 0.17 \\
\hline Maximum = & 3.76 & 3.47 & 3.96 & & 3.26 & & 3.5 & 04 Inductively coupled plasma & $n=$ & 55 \\
\hline Median $=$ & & 3.28 & 3.16 & & & & & 05 Direct current plasma & $\mathrm{Uh}=$ & 3.35 \\
\hline F-pseudosigma $=$ & & 0.09 & 0.29 & & & & & $\begin{array}{l}06 \text { Inductively coupled plasma/mass spectrometry } \\
07 \text { Ion chromatography } \\
12 \text { Flame emission }\end{array}$ & $\operatorname{Lh}=$ & 3.12 \\
\hline
\end{tabular}

\begin{tabular}{|c|c|c|c|c|c|c|c|c|c|c|c|c|c|c|c|c|c|c|c|}
\hline \multicolumn{10}{|c|}{ Method Codes } & \multirow[b]{2}{*}{ Lab } & \multirow[b]{2}{*}{ Rating } & \multirow[b]{2}{*}{$Z$-value } & \multicolumn{7}{|c|}{ Method Codes } \\
\hline Lab & Rating & Z-value & 0 & 1 & 4 & 5 & 6 & 7 & 12 & & & & 0 & 1 & 4 & 5 & 6 & 7 & 12 \\
\hline 1 & 3 & -0.53 & - & $\overline{3.11}$ & - & -- & $=$ & 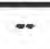 & - & 259 & 2 & -1.06 & - & $\overline{--}$ & 3.02 & - & $\cdots$ & -- & -- \\
\hline 5 & 0 & -12.90 & - & .. & $<1.00$ & - & - & - & -- & 265 & 4 & -0.29 & - & - & 3.15 & -- & -- & - & - \\
\hline 8 & 2 & 1.17 & - & - & 3.4 & - & - & - & - & 266 & 1 & 1.76 & - & - & - & - & - & - & 3.5 \\
\hline 10 & 3 & 0.59 & - & 3.3 & - & -. & - & - & - & 279 & 0 & 3.28 & 3.76 & - & -. & -. & - & - & -- \\
\hline 16 & 4 & 0.00 & - & - & 3.2 & - & -- & -. & - & 305 & 0 & 4.46 & -- & - & 3.96 & - & -- & - & - \\
\hline 23 & 4 & -0.47 & - & 3.12 & - & - & $=$ & $=$ & $\cdots$ & 315 & 3 & 0.59 & $\cdots$ & $\cdots$ & 3.3 & - & - & 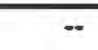 & - \\
\hline 24 & 3 & 0.70 & - & -- & 3.32 & - & - & -- & - & 321 & 2 & 1.17 & -. & 3.4 & -- & - & - & - & -. \\
\hline 25 & 0 & -12.96 & - & -- & 0.99 & - & - & .. & - & 328 & 0 & -9.97 & - & -- & 1.5 & -- & - & - & .- \\
\hline 26 & 4 & -0.47 & - & - & 3.12 & + & -- & - & - & 330 & 2 & 1.23 & - & -- & 3.41 & -- & -. & - & .. \\
\hline 32 & 2 & -1.29 & - & - & -- & -- & 2.98 & - & - & 333 & 0 & 4.28 & -. & - & 3.93 & - & - & - & .. \\
\hline 33 & 3 & 0.53 & - & - & -. & 3.29 & $=$ & - & - & 336 & 0 & -14.14 & 0.79 & $=$ & $-\cdot$ & - & - & - & -- \\
\hline 38 & 3 & 0.65 & - & 3.31 & -. & - & -. & -. & - & 341 & 4 & 0.00 & -- & 3.2 & - & -. & - & - & -. \\
\hline 42 & 2 & -1.11 & - & -- & 3.01 & - & -- & -. & - & 366 & 4 & 0.35 & - & -- & 3.26 & -. & - & - & -- \\
\hline 45 & 4 & 0.29 & - & -- & -. & - & 3.25 & -. & - & 370 & 0 & 2.93 & - & -. & 3.7 & - & - & - & -- \\
\hline 46 & 3 & -0.88 & $=$ & -. & 3.05 & - & - & - & - & 372 & 0 & -2.58 & - & - & 2.76 & $=$ & - & - & .. \\
\hline 59 & 3 & 0.59 & $\overline{-}$ & 3.3 & - & - & $=$ & $=$ & - & 375 & 4 & -0.04 & $=$ & 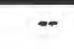 & -- & - & $\cdots$ & 3.193 & $\cdots$ \\
\hline 64 & 4 & 0.18 & - & 3.23 & -. & - & -- & .. & - & & & & & & & & & & \\
\hline 70 & 4 & -0.12 & - & - & 3.18 & - & -- & -. & - & & & & & & & & & & \\
\hline 76 & 4 & 0.35 & - & -- & - & $=$ & 3.26 & - & - & & & & & & & & & & \\
\hline 85 & 1 & 1.58 & - & 3.47 & -- & - & - & - & - & & & & & & & & & & \\
\hline 86 & 2 & 1.23 & - & - & 3.41 & $\rightarrow$ & - & $=$ & - & & & & & & & & & & \\
\hline 89 & 3 & 1.00 & - & 3.37 & - & - & - & .. & - & & & & & & & & & & \\
\hline 97 & 4 & 0.00 & - & 3.2 & -. & - & - & -- & - & & & & & & & & & & \\
\hline 102 & 0 & -9.74 & - & -- & 1.54 & - & -. & - & -. & & & & & & & & & & \\
\hline 105 & 2 & 1.47 & - & -. & 3.45 & - & $\cdots$ & $=$ & $\because$ & & & & & & & & & & \\
\hline 109 & 4 & 0.00 & - & 3.2 & -- & - & -- & $\overrightarrow{-}$ & -. & & & & & & & & & & \\
\hline 113 & 4 & -0.23 & - & .. & 3.16 & - & - & - & -- & & & & & & & & & & \\
\hline 134 & 4 & 0.29 & - & 3.25 & -- & -- & - & -- & - & & & & & & & & & & \\
\hline 138 & 4 & -0.47 & - & -- & 3.12 & - & - & -. & - & & & & & & & & & & \\
\hline 142 & 4 & -0.23 & - & - & 3.16 & - & -- & - & - & & & & & & & & & & \\
\hline 146 & 0 & 3.70 & $=$ & $\ldots$ & 3.83 & $=$ & $\cdots$ & -- & - & & & & & & & & & & \\
\hline 180 & 4 & -0.35 & - & -- & 3.14 & - & - & - & -- & & & & & & & & & & \\
\hline 190 & 4 & 0.47 & - & 3.28 & - & - & - & - & - & & & & & & & & & & \\
\hline 193 & 3 & 0.70 & - & 3.32 & - & - & -. & - & - & & & & & & & & & & \\
\hline 212 & 0 & 2.35 & - & . & 3.6 & - & - & - & - & & & & & & & & & & \\
\hline 224 & 2 & -1.26 & $\because$ & - & 2.986 & - & 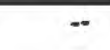 & $=$ & 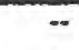 & & & & & & & & & & \\
\hline 234 & 4 & -0.29 & - & .- & 3.15 & -. & -. & - & -. & & & & & & & & & & \\
\hline 245 & 3 & -0.76 & -- & -. & 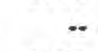 & $\cdots$ & 3.071 & - & -. & & & & & & & & & & \\
\hline 247 & 2 & -1.29 & - & - & 2.98 & -- & -- & - & - & & & & & & & & & & \\
\hline 256 & 2 & -1.06 & - & -. & -- & -- & -- & - & 3.02 & & & & & & & & & & \\
\hline
\end{tabular}


M-162 MAGNESIUM (Mg) In mg/L

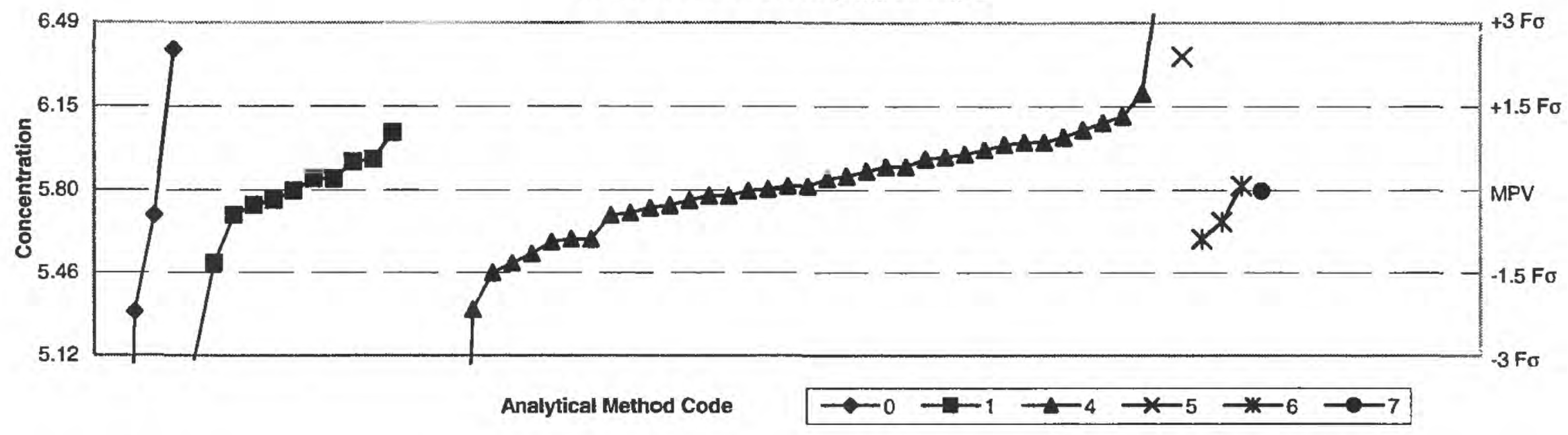

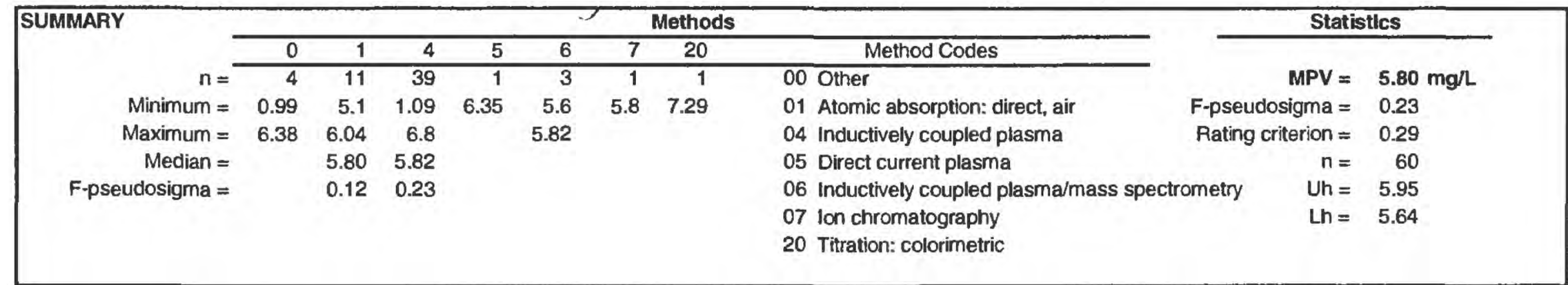

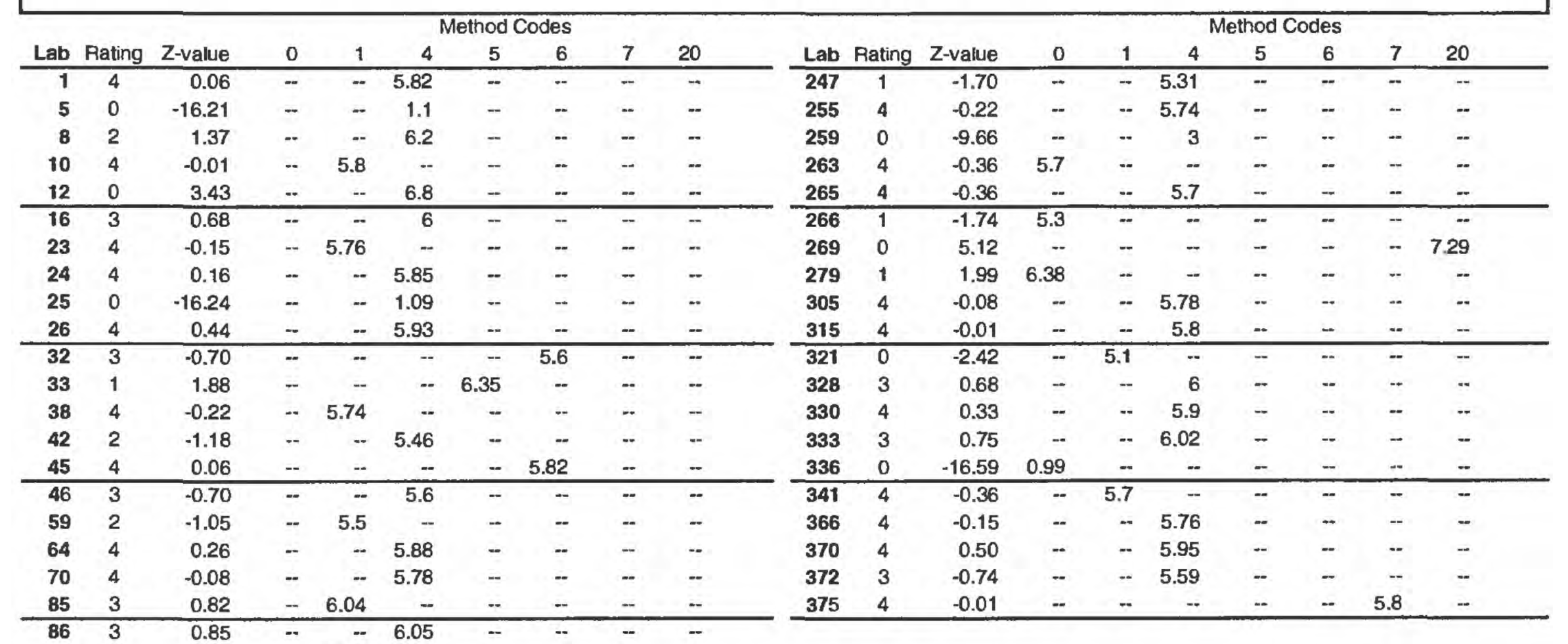


M-162 SODIUM ( $\mathrm{Na})$ in $\mathrm{mg} / \mathrm{L}$

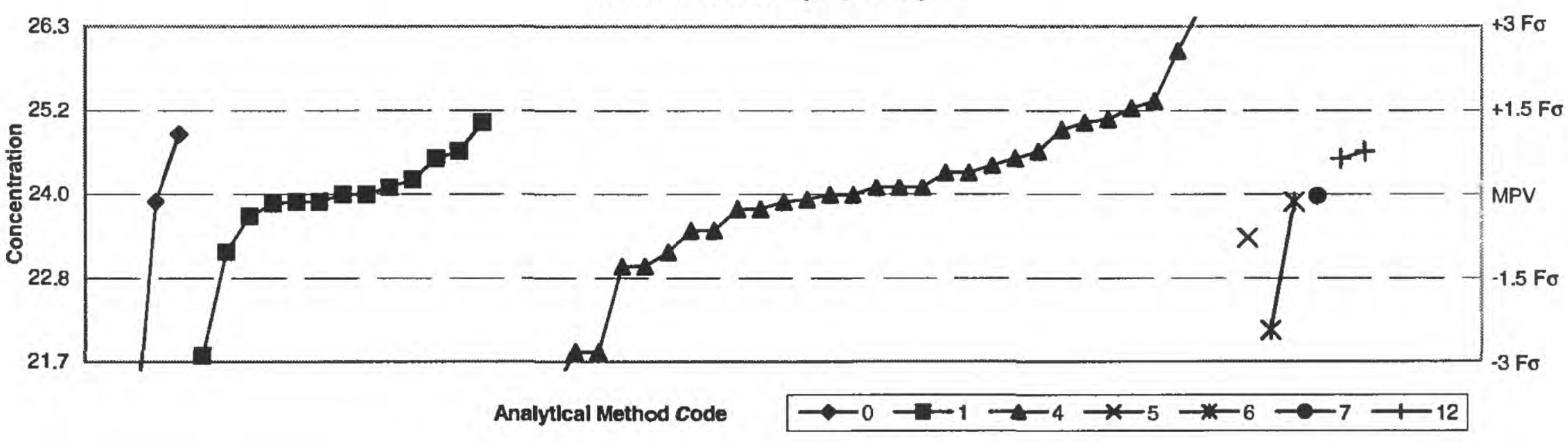

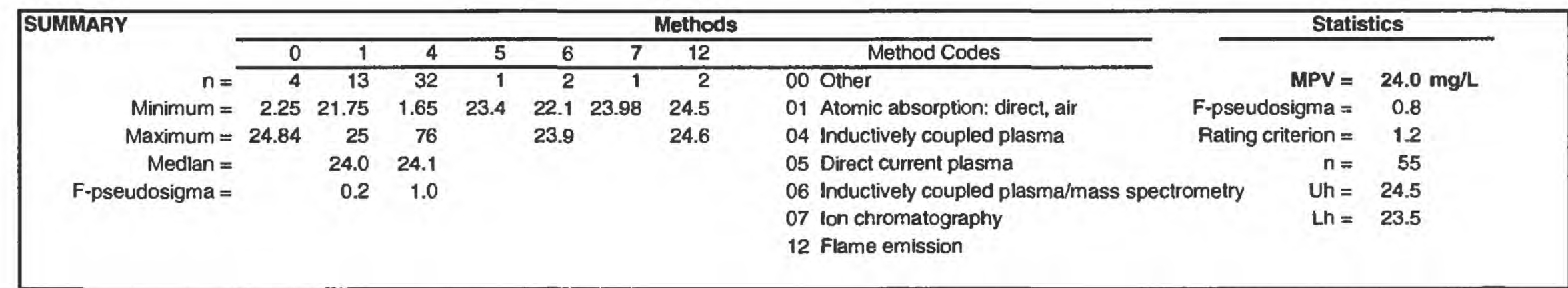

\begin{tabular}{|c|c|c|c|c|c|c|c|c|c|c|c|c|c|c|c|c|c|c|c|}
\hline \multicolumn{10}{|c|}{ Method Codes } & & & & \multicolumn{7}{|c|}{ Method Codes } \\
\hline Lab & Rating & $Z$-value & 0 & 1 & 4 & 5 & 6 & 7 & 12 & Lab & Rating & Z-value & 0 & 1 & 4 & 5 & 6 & 7 & 12 \\
\hline 1 & 4 & -0.06 & $\overline{--}$ & - & 23.93 & - & $=$ & -. & 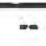 & 256 & 4 & 0.42 & - & - & 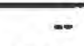 & - & - & -- & 24.5 \\
\hline 5 & 0 & -18.53 & .- & - & 1.76 & -- & -. & -. & - & 259 & 4 & 0.42 & .- & -. & 24.5 & .. & -- & -. & .. \\
\hline 8 & 4 & 0.08 & -. & - & 24.1 & -- & -- & -. & - & 265 & 4 & 0.00 & - & -. & 24 & -. & -. & -. & -. \\
\hline 10 & 4 & 0.00 & - & 24 & -. & -- & - & .. & -- & 266 & 4 & 0.50 & .. & -- & -. & - & -- & -- & 24.6 \\
\hline 12 & 1 & 1.67 & -. & - & 26 & -- & - & -. & - & 279 & 3 & 0.70 & 24.84 & - & - & -. & - & -- & -. \\
\hline 16 & $\overline{3}$ & -0.83 & - & - & 23 & $=$ & - & -. & -- & 305 & $\overline{0}$ & -2.33 & - & $\overline{-}$ & 21.2 & -- & - & -- & - \\
\hline 23 & 4 & -0.08 & -- & 23.9 & .. & - & - & .. & - & 315 & 4 & -0.08 & 23.9 & - & -- & - & .- & -- & -. \\
\hline 24 & 4 & 0.25 & -. & - & 24.3 & - & - & -. & - & 321 & 0 & -3.25 & 20.1 & - & - & .- & .- & -- & -. \\
\hline 25 & 0 & -18.63 & .. & - & 1.65 & - & -- & -- & - & 328 & 3 & 0.83 & - & -. & 25 & - & - & - & -- \\
\hline 26 & 4 & 0.25 & -- & -- & 24.3 & - & - & .. & - & 330 & 3 & 1.00 & -. & - & 25.2 & -. & .. & .. & - \\
\hline 32 & 7 & -1.58 & -- & - & - & - & 22.1 & - & - & 336 & 0 & -18.13 & 2.25 & - & -- & - & - & -- & - \\
\hline 33 & 4 & -0.50 & - & - & -- & 23.4 & -- & .. & -. & 341 & 3 & 0.83 & - & 25 & -. & -. & -. & - & -. \\
\hline 38 & 4 & -0.25 & -. & 23.7 & -- & - & .- & .. & -. & 366 & 4 & -0.42 & -. & -- & 23.5 & -. & .. & -- & -- \\
\hline 42 & 1 & -1.83 & .- & -. & 21.8 & -. & -. & -- & - & 370 & 0 & 2.17 & $\ldots$ & -. & 26.6 & -- & -. & - & -. \\
\hline 45 & 4 & -0.08 & -- & - & -- & -- & 23.9 & -- & - & 375 & 4 & -0.02 & - & -- & -- & - & -. & 23.98 & .. \\
\hline 46 & 4 & 0.08 & -. & - & 24.1 & - & - & - & - & & & & & & & & & & \\
\hline 59 & 4 & -0.08 & -. & 23.9 & - & -- & - & -. & -. & & & & & & & & & & \\
\hline 64 & 4 & 0.08 & -. & 24.1 & -- & - & -. & -. & -. & & & & & & & & & & \\
\hline 70 & 4 & 0.50 & -- & -- & 24.6 & -. & - & .. & -. & & & & & & & & & & \\
\hline 85 & 4 & 0.42 & -. & 24.5 & -- & -- & - & - & - & & & & & & & & & & \\
\hline 86 & 2 & 1.08 & 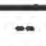 & - & 25.3 & - & - & 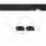 & - & & & & & & & & & & \\
\hline 89 & 4 & 0.17 & -. & 24.2 & -- & - & - & -. & - & & & & & & & & & & \\
\hline 97 & 3 & -0.67 & -. & 23.2 & -. & - & -. & -. & -. & & & & & & & & & & \\
\hline 102 & 0 & 43.33 & -. & - & 76 & -. & -- & -- & -- & & & & & & & & & & \\
\hline 105 & 4 & -0.17 & .- & - & 23.8 & -- & .- & .. & -- & & & & & & & & & & \\
\hline 109 & $\overline{1}$ & -1.88 & -. & 21.75 & - & $\overline{-}$ & $\cdots$ &.- & $=$ & & & & & & & & & & \\
\hline 113 & 3 & -0.67 & -. & -- & 23.2 & - & -. & -. & -- & & & & & & & & & & \\
\hline 121 & 3 & -0.83 & .. & - & 23 & -- & -. & .. & - & & & & & & & & & & \\
\hline 134 & 4 & -0.11 & -- & 23.87 & - & -- & .- & -. & -- & & & & & & & & & & \\
\hline 138 & 4 & -0.17 & .- & - & 23.8 & -. & .. & .. & -. & & & & & & & & & & \\
\hline 142 & 4 & -0.08 & $\cdots$ & - & 23.9 & - & $\cdots$ & - & - & & & & & & & & & & \\
\hline 146 & 3 & 0.75 & -. & - & 24.9 & - & -. & -. & -- & & & & & & & & & & \\
\hline 180 & 4 & 0.00 & -- & - & 24 & -- & -- & .- & -- & & & & & & & & & & \\
\hline 190 & 4 & 0.50 & - & 24.6 & .- & -- & -. & -. & -. & & & & & & & & & & \\
\hline 193 & 4 & 0.00 & -. & 24 & .. & - & -. & .. & - & & & & & & & & & & \\
\hline 212 & 4 & -0.42 & -. & - & 23.5 & - & - & 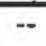 & - & & & & & & & & & & \\
\hline 220 & 4 & 0.08 & .. & -- & 24.1 & - & .- & -. & -. & & & & & & & & & & \\
\hline 224 & 3 & 0.87 & .. & - & 25.05 & -- & .. & -. & - & & & & & & & & & & \\
\hline 234 & 4 & 0.33 & -- & - & 24.4 & -. & -. & - & -. & & & & & & & & & & \\
\hline 247 & 1 & -1.83 & - & - & 21.8 & - & - & -- & - & & & & & & & & & & \\
\hline
\end{tabular}


Table 12. Statistical summary of reported data for standard reference sample M-162 (major constituents) - continued

M-162 pH

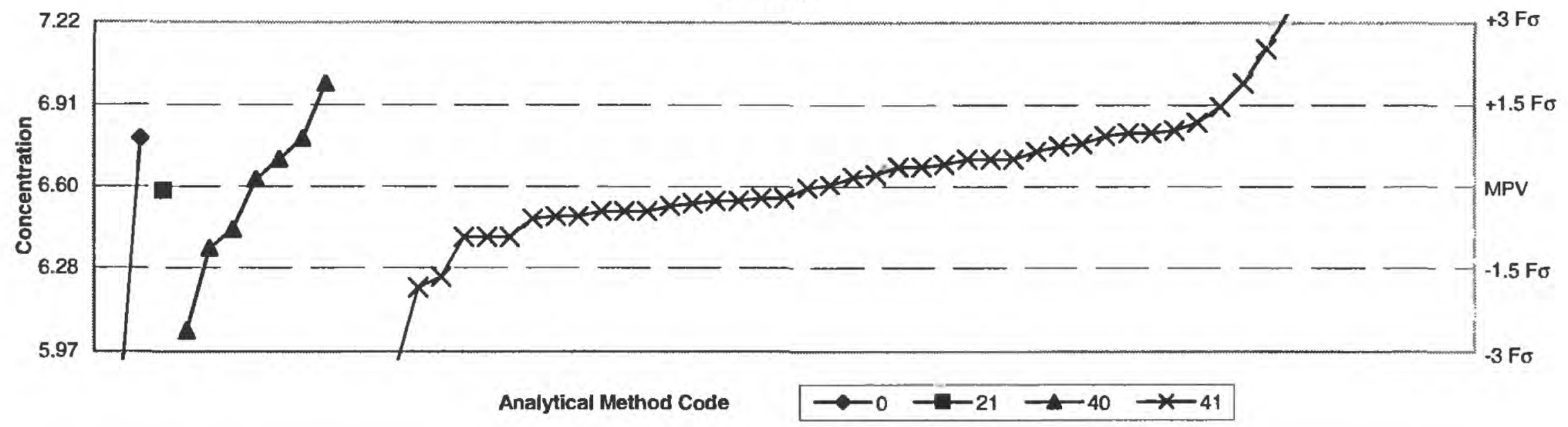

\begin{tabular}{rlrrrrl}
\hline SUMMARY & & & & & Methods \\
\cline { 2 - 7 }$n$ & 0 & 21 & 40 & 41 & Method Codes \\
Minimum & $=$ & 5.68 & 6.58 & 6.05 & 5.75 & 21 Titration: electromelric \\
Maximum & $=$ & 6.78 & & 6.99 & 7.27 & 40 Ion selective electrode \\
Median & $=$ & & & 6.62 & 6.60 & 41 Electrometric \\
F-pseudosigma & $=$ & & 0.26 & 0.20 &
\end{tabular}

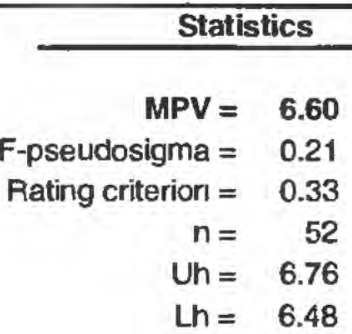

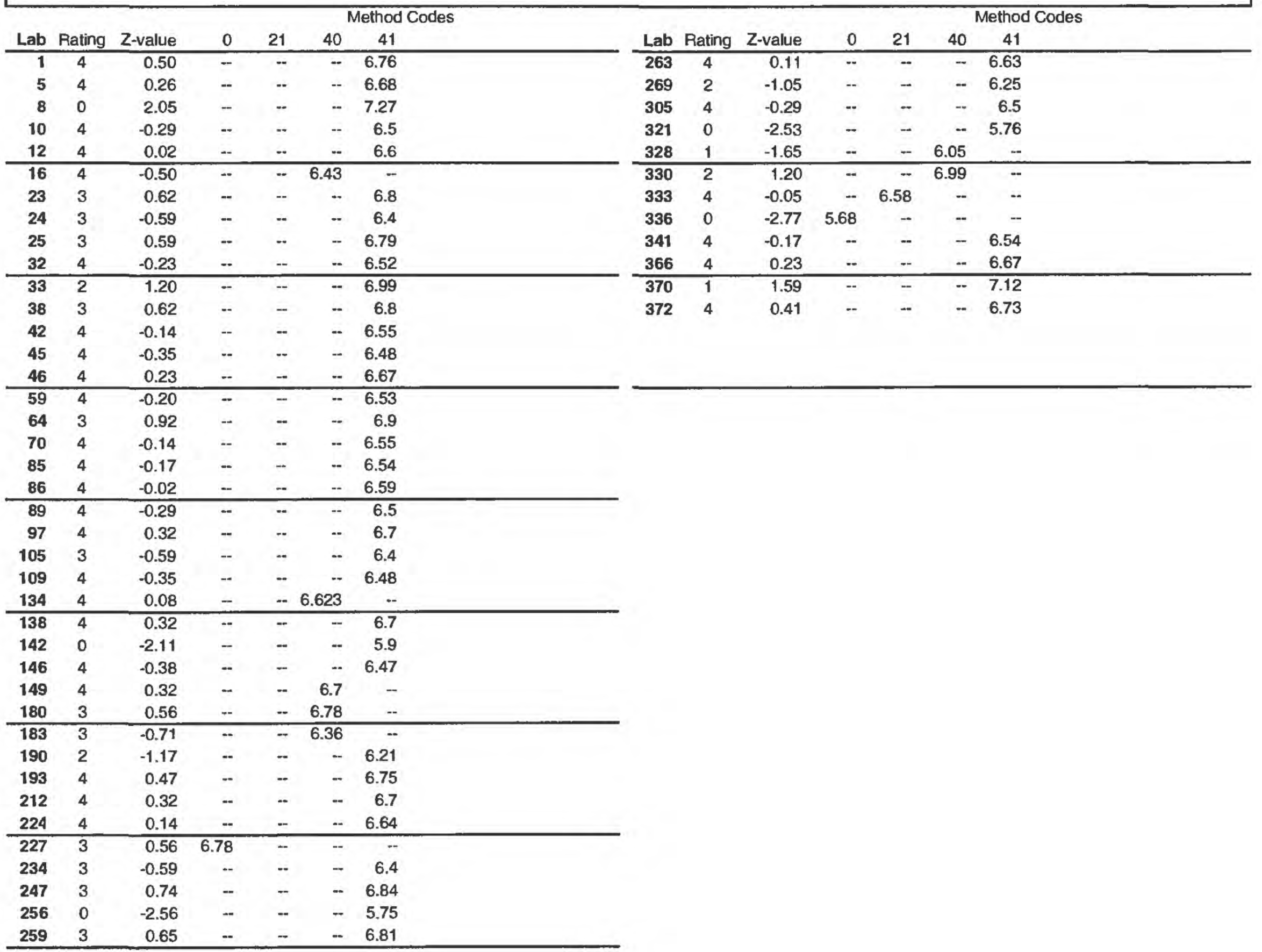


Table 12. Statistical summary of reported data for standard reference sample M-162 (major constituents) -- continued M-162 ROE (Residue on Evaporation) in $\mathrm{mg} / \mathrm{L}$

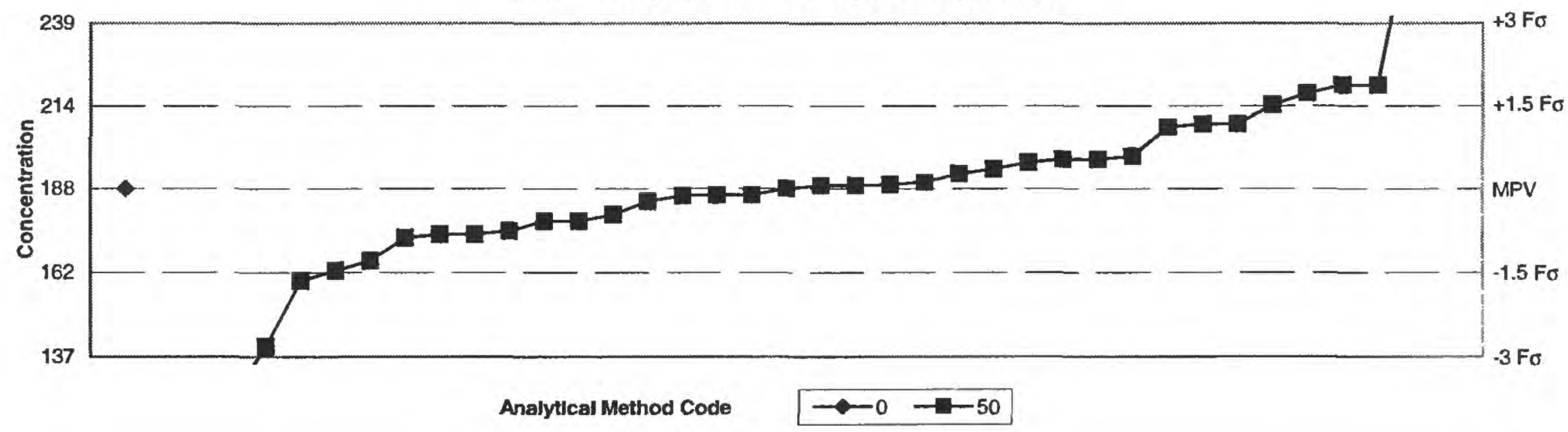

\begin{tabular}{|c|c|c|c|c|c|}
\hline \multirow[t]{2}{*}{ SUMMARY } & \multicolumn{3}{|c|}{ Methods } & \multicolumn{2}{|c|}{ Statistics } \\
\hline & 0 & 50 & Method Codes & & \\
\hline$n=$ & 1 & 37 & 00 Other & MPV $=$ & $188 \mathrm{mg} / \mathrm{L}$ \\
\hline Minimum $=$ & 188 & 58 & 50 Gravimetric & F-pseudosigma $=$ & 17 \\
\hline Maximum $=$ & & 272 & & $n=$ & 38 \\
\hline Median $=$ & & 188 & & $\mathrm{Uh}=$ & 197 \\
\hline F-pseudosigma $=$ & & 17 & & $\operatorname{Lh}=$ & 174 \\
\hline
\end{tabular}

\begin{tabular}{|c|c|c|c|c|}
\hline \multirow[b]{2}{*}{ Lab } & \multirow[b]{2}{*}{ Rating } & \multirow[b]{2}{*}{$Z$-value } & \multirow{2}{*}{\multicolumn{2}{|c|}{ Method Codes }} \\
\hline & & & & \\
\hline 1 & 4 & -0.23 & $-\quad 184$ & \\
\hline 5 & 4 & 0.47 & - 196 & \\
\hline 8 & 4 & 0.07 & -189.2 & \\
\hline 10 & 3 & -0.82 & - $\quad 174$ & \\
\hline 12 & 0 & -7.62 & $\begin{array}{l}. \\
\ldots\end{array}$ & \\
\hline 16 & 0 & -4.05 & $--\quad 119$ & \\
\hline 25 & 0 & 4.93 & - $\quad 272$ & \\
\hline 26 & 3 & -0.59 & .. $\quad 178$ & \\
\hline 32 & 1 & 1.52 & -- 214 & \\
\hline 46 & 4 & -0.12 & - $\quad 186$ & \\
\hline 59 & 3 & 0.53 & 197 & \\
\hline 70 & 4 & 0.12 & $\begin{array}{l}-\quad 190 \\
-\end{array}$ & \\
\hline 85 & 2 & -1.47 & -- $\quad 163$ & \\
\hline 89 & 2 & -1.29 & $-\quad 166$ & \\
\hline 105 & 1 & 1.88 & $-\quad 220$ & \\
\hline 109 & 4 & 0.06 & 189 & \\
\hline 113 & 4 & -0.13 & - 185.7 & \\
\hline 134 & 4 & 0.35 & -. 194 & \\
\hline 138 & 3 & -0.59 & -- $\quad 178$ & \\
\hline 142 & 3 & -0.88 & 173 & \\
\hline 146 & 2 & 1.17 & 208 & \\
\hline 190 & 3 & 0.53 & $\begin{array}{l}-\quad 197 \\
-\end{array}$ & \\
\hline 212 & 4 & -0.47 & -- $\quad 180$ & \\
\hline 224 & 1 & 1.73 & -217.5 & \\
\hline 227 & 4 & 0.00 & $\begin{array}{l}- \\
-\end{array}$ & \\
\hline 234 & 0 & -3.81 & 123 & \\
\hline 247 & 3 & -0.82 & $\begin{array}{l}-\quad 174 \\
\end{array}$ & \\
\hline 256 & 3 & 0.59 & $\begin{array}{l}-\quad 198 \\
-\end{array}$ & \\
\hline 263 & 3 & -0.76 & 175 & \\
\hline 266 & 4 & 0.00 & 188 & \\
\hline 277 & 4 & 0.06 & 189 & \\
\hline 328 & 0 & -2.82 & 140 & \\
\hline 330 & 4 & -0.12 & 186 & \\
\hline 341 & 2 & 1.17 & -- 208 & \\
\hline 356 & 4 & 0.27 & -- 192.6 & \\
\hline 366 & 1 & -1.64 & -160 & \\
\hline 370 & 1 & 1.88 & 220 & \\
\hline 372 & 2 & 1.11 & 207 & \\
\hline
\end{tabular}


Table 12. Statistical summary of reported data for standard reference sample M-162 (major constituents) -- continued

M-162 SILICA $\left(\mathrm{SiO}_{2}\right)$ in $\mathrm{mg} / \mathrm{L}$

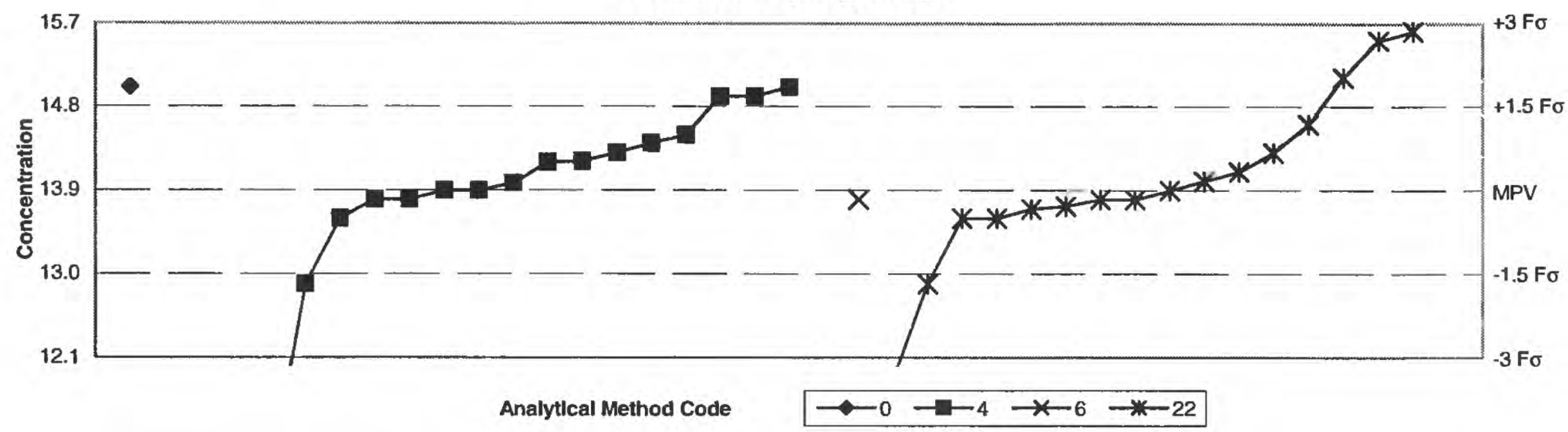

\begin{tabular}{|c|c|c|c|c|c|c|c|c|}
\hline \multirow[t]{2}{*}{ SUMMARY } & \multicolumn{6}{|c|}{ Methods } & \multicolumn{2}{|c|}{ Statistics } \\
\hline & 0 & 4 & 5 & 6 & 22 & Method Codes & \multirow[b]{2}{*}{$M P V=$} & \multirow[b]{2}{*}{$13.9 \mathrm{mg} / \mathrm{L}$} \\
\hline$n=$ & 1 & 19 & 1 & 1 & 16 & 00 Other & & \\
\hline Minimum $=$ & 15 & 0.547 & 6.12 & 13.8 & 11.88 & 04 Inductively coupled plasma & \multirow{2}{*}{$\begin{array}{r}\text { F-pseudosigma = } \\
\text { Rating criterion }=\end{array}$} & 0.6 \\
\hline Maximum $=$ & & 15 & & & 15.6 & 05 Direct current plasma & & 0.7 \\
\hline Median $=$ & & 13.9 & & & 13.9 & 06 Inductively coupled plasma/mass spectrometry & $n=$ & 38 \\
\hline \multirow[t]{2}{*}{ F-pseudosigma $=$} & & 0.8 & & & 0.6 & 22 Colorimetric & $\mathrm{Uh}=$ & 14.4 \\
\hline & & & & & & & $\operatorname{Lh}=$ & 13.6 \\
\hline
\end{tabular}

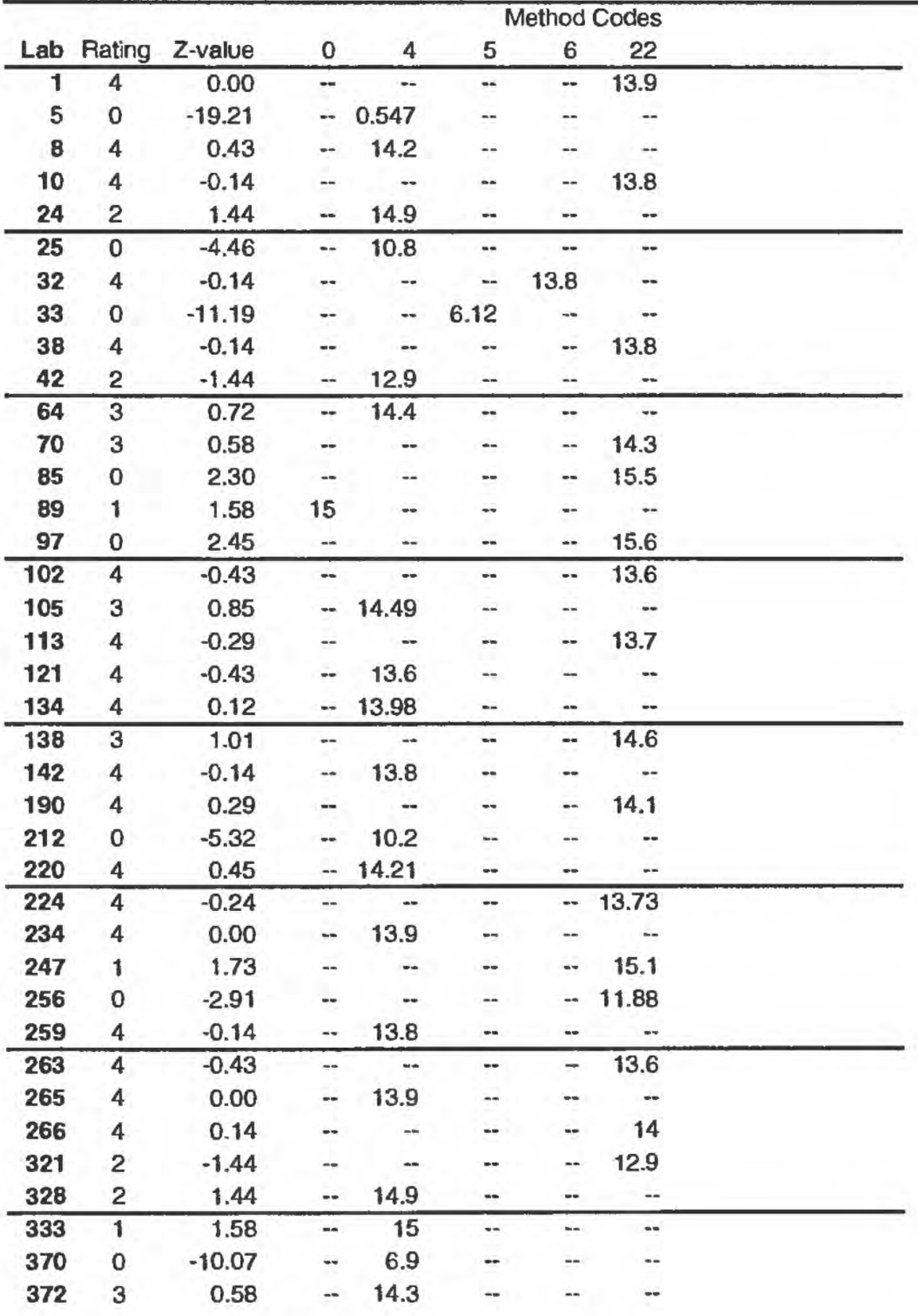


Table 12. Statistical summary of reported data for standard reference sample $\mathbf{M - 1 6 2}$ (major const/tuents) -- continued M-162 SULFATE $\left(\mathrm{SO}_{4}\right)$ In $\mathrm{mg} / \mathrm{L}$

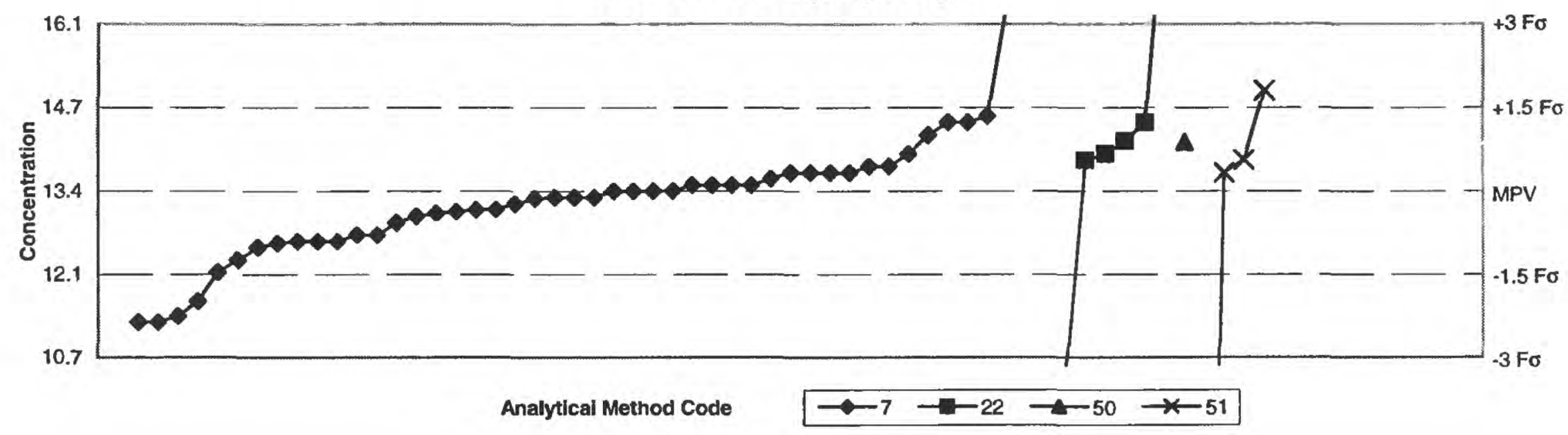

\begin{tabular}{|c|c|c|c|c|c|c|c|c|}
\hline \multirow[t]{2}{*}{ SUMMARY } & \multicolumn{6}{|c|}{ Methods } & \multicolumn{2}{|c|}{ Statistics } \\
\hline & 0 & 7 & 22 & 50 & 51 & Method Codes & & \\
\hline$n=$ & 1 & 45 & 8 & 1 & 4 & 00 Other & MPV $=$ & $13.4 \mathrm{mg} / \mathrm{L}$ \\
\hline Minimum $=$ & 0.999 & 11.3 & 4 & 14.19 & 1.3 & 07 lon chromatography & F.pseudosigma = & 0.9 \\
\hline Maximum = & & 18.43 & 18.2 & & 15 & 22 Colorimetric & $n=$ & 59 \\
\hline Median $=$ & & 13.3 & 14.0 & & & 50 Gravimetric & $\mathrm{Un}=$ & 13.8 \\
\hline F-pseudosigma $=$ & & 0.7 & 3.4 & & & 51 Turbidimetric & $\operatorname{Ln}=$ & 12.6 \\
\hline
\end{tabular}

\begin{tabular}{|c|c|c|c|c|c|c|c|c|c|c|c|c|c|c|c|}
\hline \multicolumn{11}{|c|}{ Method Codes } & \multicolumn{5}{|c|}{ Method Codes } \\
\hline Lab & Rating & Z-value & 0 & 7 & 22 & 50 & 51 & Lab & Rating & $Z$-value & 0 & 7 & 22 & 50 & 51 \\
\hline 1 & 4 & -0.13 & $\ldots$ & 13.28 & -- & - & - & 247 & 3 & -0.79 & - & 12.7 & - & - & - \\
\hline 4 & 0 & -2.36 & -- & 11.3 & - & -- & -- & 255 & 3 & 0.67 & - & -. & 14 & 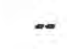 & - \\
\hline 5 & 4 & -0.39 & - & 13.05 & -- & -. & - & 256 & 0 & 3.41 & - & 16.43 & - &.- & - \\
\hline 8 & 3 & -0.90 & - & 12.6 & - & - & -- & 259 & 4 & 0.00 & - & 13.4 & - &.- & - \\
\hline 10 & 4 & 0.34 & - & - & - & -. & 13.7 & 263 & 3 & 0.56 & - & -. & - & -. & 13.9 \\
\hline 12 & 0 & -10.57 & - & - & 4 & - & -- & 265 & 2 & 1.24 & - & 14.5 & -. & - & - \\
\hline 16 & 2 & 1.01 & -- & 14.3 & -. & - & -- & 266 & 1 & 1.80 & - & -- & -- & -. & 15 \\
\hline 23 & 0 & -13.60 & -. & - & -. & - & 1.3 & 277 & 4 & -0.22 & -- & 13.2 & - & -. & -- \\
\hline 24 & 3 & 0.90 & - & -. & 14.2 & - & -- & 305 & 3 & -0.93 & -- & 12.57 & -- & - & - \\
\hline 25 & 2 & -1.46 & .. & 12.1 & -- & -. & - & 315 & 4 & 0.34 & - & 13.7 & - & - & - \\
\hline 26 & 2 & -1.01 & -- & 12.5 & -- & - & $\ldots$ & 321 & 1 & -1.98 & - & 11.64 & $=$ & $\cdots$ & - \\
\hline 32 & 4 & 0.45 & -. & 13.8 & - & - & $\cdots$ & 328 & 4 & -0.45 & - & 13 & -- & - & - \\
\hline 33 & 2 & 1.24 & - & 14.5 & - & - & - & 330 & 0 & 5.40 & - & -. & 18.2 & -. & - \\
\hline 42 & 0 & -2.36 & -. & 11.3 & -- & -- & - & 336 & 0 & -13.94 & 0.999 & - & - & - & - \\
\hline 45 & 3 & -0.56 & - & 12.9 & .. & -. & - & 341 & 0 & -3.26 & - & .. & 10.5 & -. & $\ldots$ \\
\hline 46 & 4 & -0.11 & -. & 13.3 & -- & - & - & 356 & 4 & -0.34 & - & 13.1 & -- & - & - \\
\hline 59 & 4 & 0.45 & - & 13.8 & - & - & - & 366 & 3 & 0.56 & - & .. & 13.9 & -. & - \\
\hline 64 & 4 & 0.34 & -- & 13.7 & - & - & $\cdots$ & 370 & 2 & 1.35 & -. & 14.6 & -- & -- & -. \\
\hline 70 & 4 & 0.22 & - & 13.6 & -. & - & - & 372 & 0 & -2.25 & -. & 11.4 & - & - & -- \\
\hline 85 & 3 & 0.67 & -- & 14 & - & - & - & & & & & & & & \\
\hline
\end{tabular}


Table 12. Statistical summary of reported data for standard reference sample M-162 (major constituents) -- continued

M-162 SPECIFIC CONDUCTANCE in $\mu \mathrm{S} / \mathrm{cm}$

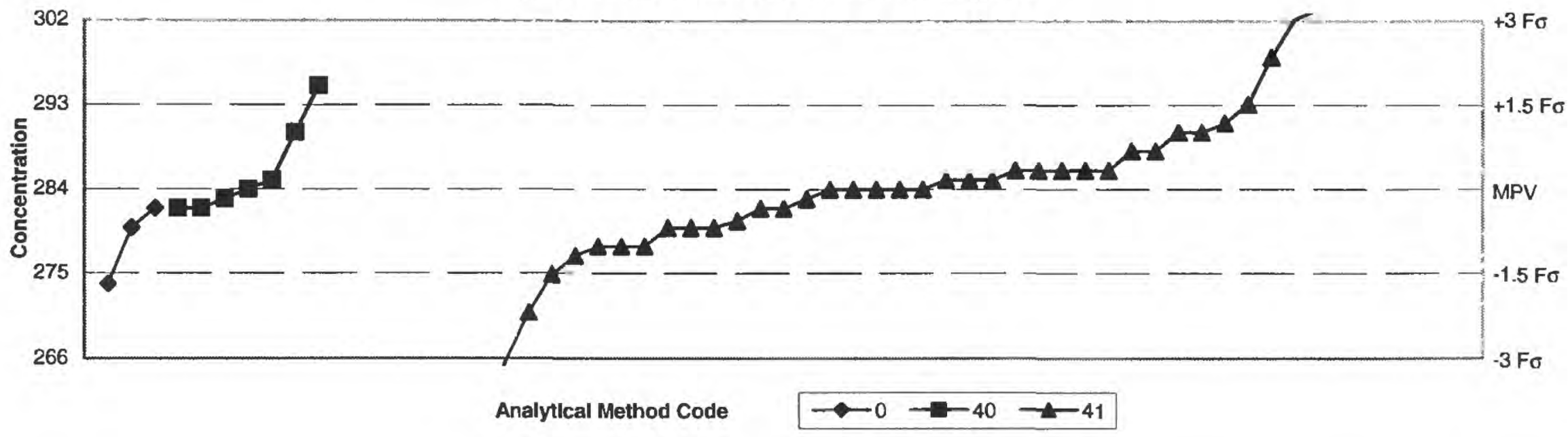

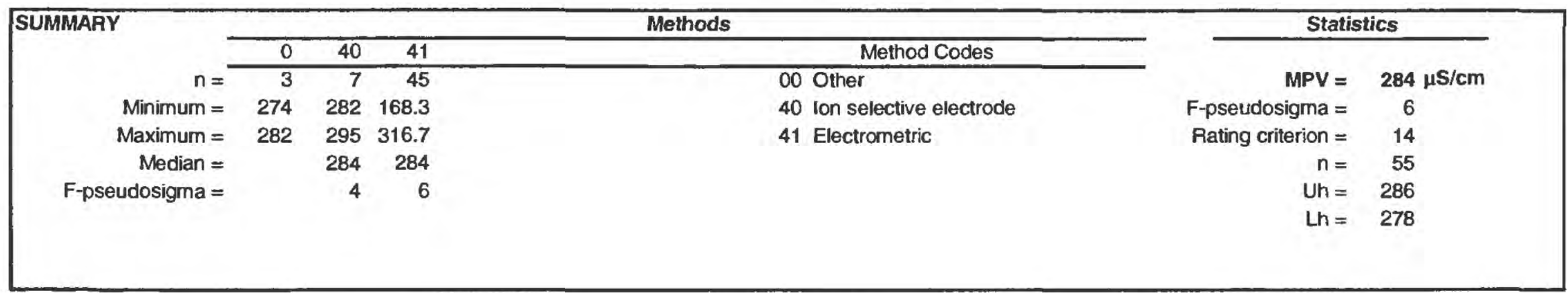

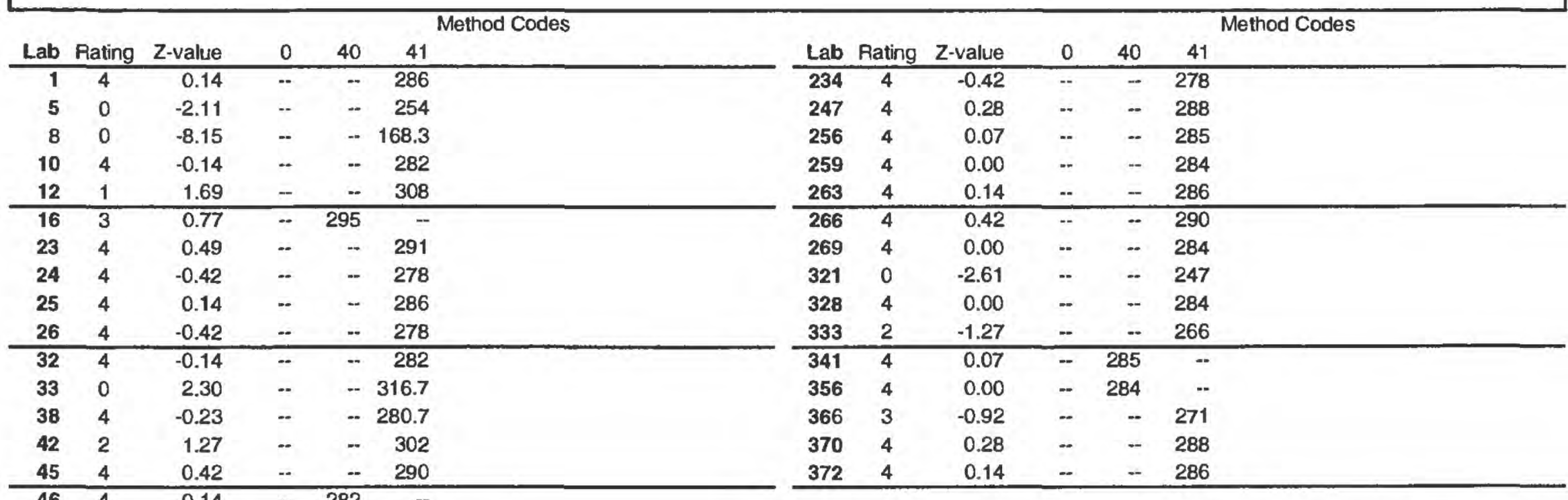


Table 12. Statistical summary of reported data for standard reference sample M-162 (major constituents) -- continued

M-162 STRONTIUM (Sr) in $\mu \mathrm{g} / \mathrm{L}$

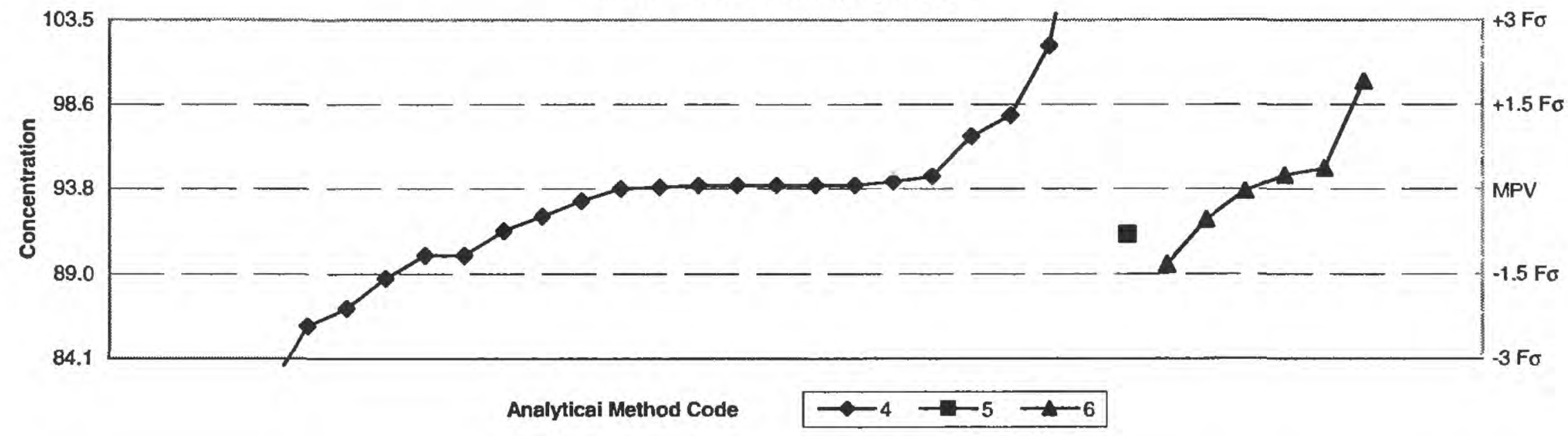

\begin{tabular}{|c|c|c|c|c|c|c|c|}
\hline \multirow[t]{2}{*}{ SUMMARY } & \multicolumn{5}{|c|}{ Methods } & \multicolumn{2}{|c|}{ Statistics } \\
\hline & 2 & 4 & 5 & 6 & Method Codes & & \\
\hline$n=$ & $\overline{0}$ & 24 & 1 & $\overline{6}$ & 02 Atomic absorption: direct, nitrous oxide & MPV $=$ & $93.8 \mu \mathrm{g} / \mathrm{L}$ \\
\hline Minimum $=$ & 0 & 23.7 & 91.2 & 89.5 & 04 Inductively coupled plasma & F-pseudosigma $=$ & 3.2 \\
\hline Maximum $=$ & & 113 & & 100 & 05 Direct current plasma & Rating criterion = & 4.7 \\
\hline Median $=$ & & 93.8 & & 94.2 & 06 Inductively coupled plasma/mass spectrometry & $\mathrm{n}=$ & 31 \\
\hline \multirow[t]{2}{*}{ F-pseudosigma $=$} & & 3.5 & & 2.2 & & $\mathrm{Uh}=$ & 94.4 \\
\hline & & & & & & $\mathrm{Lh}=$ & 90.0 \\
\hline
\end{tabular}

\begin{tabular}{|c|c|c|c|c|c|c|c|}
\hline \multirow[b]{2}{*}{ Lab } & \multirow[b]{2}{*}{ Rating } & \multirow[b]{2}{*}{ Z-value } & \multicolumn{5}{|c|}{ Method Codes } \\
\hline & & & 2 & 4 & 5 & 6 & \\
\hline 1 & 4 & 0.02 & - & 93.88 & -- & - & \\
\hline 5 & 0 & -14.95 & -. & 23.7 & -- & -- & \\
\hline 8 & 2 & 1.32 & - & -- & -- & 100 & \\
\hline 16 & 3 & -0.81 & .. & 90 & -- & -. & \\
\hline 24 & 3 & 0.64 & -. & 96.8 & -- & -- & \\
\hline 25 & 3 & -0.81 & 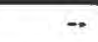 & 90 & $\cdots$ & $\overline{-}$ & \\
\hline 32 & 4 & 0.26 & -- & -- & -- & 95 & \\
\hline 33 & 3 & -0.55 & .. & -- & 91.2 & -- & \\
\hline 42 & 0 & 4.09 & -. & 113 & - & -. & \\
\hline 59 & 4 & -0.02 & -- & -- & -- & 93.7 & \\
\hline 76 & 4 & -0.37 & -. & -- & -- & 92.06 & \\
\hline 85 & 4 & 0.04 & -. & 94 & -- & -- & \\
\hline 86 & 4 & 0.04 & -. & 94 & - & -- & \\
\hline 97 & 4 & -0.34 & -. & 92.2 & -- & -- & \\
\hline 102 & 0 & -2.52 & -. & 82 & -. & - & \\
\hline 105 & 4 & 0.04 & - & 94 & - & - & \\
\hline 113 & 3 & -0.51 & - & 91.4 & -. & -- & \\
\hline 121 & 4 & 0.04 & -. & 94 & -- & -- & \\
\hline 134 & 4 & -0.15 & -- & 93.08 & -. & - & \\
\hline 138 & 4 & 0.00 & -. & 93.8 & -. & -. & \\
\hline 142 & 1 & 1.75 & - & 102 & -- & - & \\
\hline 190 & 0 & -20.00 & $<0.01$ & - & -. & -. & \\
\hline 234 & 4 & 0.15 & -- & 94.5 & -- & - & \\
\hline 245 & 3 & -0.92 & -- & -- & -- & 89.5 & \\
\hline 247 & 0 & -2.94 & - & 80 &.- & -- & \\
\hline 256 & 2 & -1.09 & -. & 88.7 & - & - & \\
\hline 259 & 3 & 0.90 & -- & 98 & - & -- & \\
\hline 265 & 4 & 0.04 & - & 94 & - & -. & \\
\hline 328 & 1 & -1.66 & -- & 86 & -- & -- & \\
\hline 333 & 4 & 0.09 & - & 94.2 & .. & - & \\
\hline 341 & 4 & 0.17 & $\overline{-}$ & -- & - & 94.6 & \\
\hline 372 & 2 & -1.45 & - & 87 & -. & -- & \\
\hline
\end{tabular}


Table 12. Statistical summary of reported data for standard reference sample $\mathbf{M - 1 6 2}$ (major constituents) -- continued

M-162 TOTAL PHOSPHORUS as PHOSPHORUS in mg/L

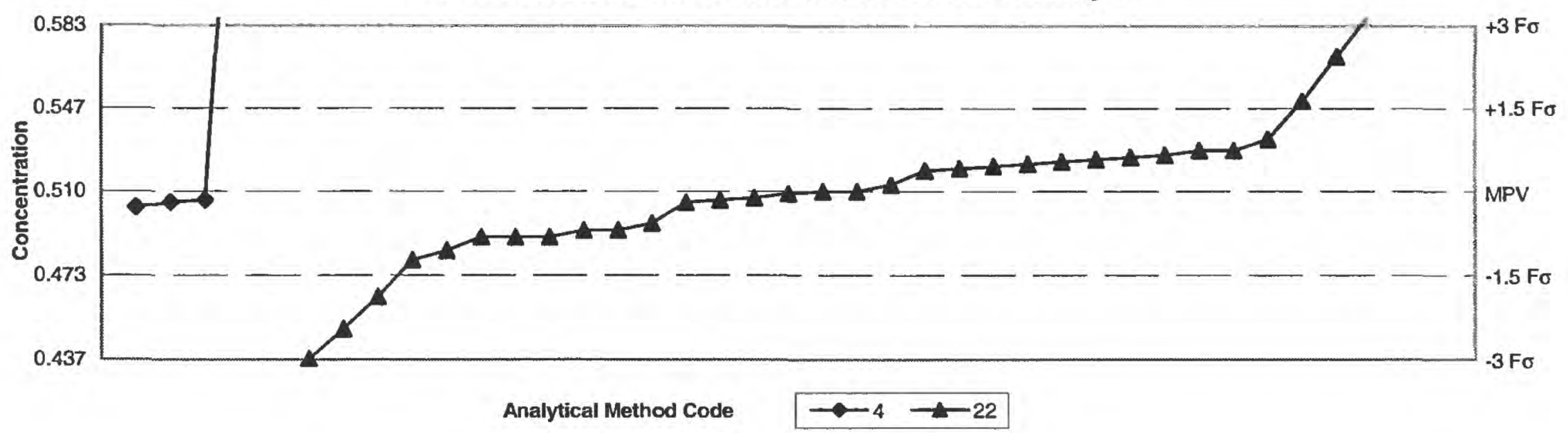

\begin{tabular}{|c|c|c|c|c|c|c|}
\hline \multirow[t]{2}{*}{ SUMMARY } & \multicolumn{4}{|c|}{ Methods } & \multicolumn{2}{|c|}{ Statistics } \\
\hline & 4 & 20 & 22 & Method Codes & & \\
\hline$n=$ & 4 & 1 & 33 & 04 Inductively coupled plasma & MPV $=$ & $0.510 \mathrm{mg} / \mathrm{L}$ \\
\hline Minimum = & 0.503 & 0.61 & 0.437 & 20 Titration: colorimetric & F-pseudosigma $=$ & 0.024 \\
\hline Maximum = & 0.72 & & 0.628 & 22 Colorimetric & Rating criterion $=$ & 0.026 \\
\hline Median $=$ & & & 0.510 & & $\mathrm{n}=$ & 38 \\
\hline \multirow[t]{2}{*}{ F-pseudosigma = } & & & 0.024 & & $\mathrm{Uh}=$ & 0.526 \\
\hline & & & & & $\operatorname{Lh}=$ & 0.493 \\
\hline
\end{tabular}

\begin{tabular}{|c|c|c|c|c|}
\hline \multirow[b]{2}{*}{ Lab } & \multirow[b]{2}{*}{ Rating } & \multirow[b]{2}{*}{ Z-value } & \multicolumn{2}{|r|}{ Method Codes } \\
\hline & & & 4 & 22 \\
\hline 5 & 4 & 0.00 & - & -0.51 \\
\hline 8 & 0 & 8.24 & 0.72 & $-\quad-$. \\
\hline 12 & 3 & -0.78 & -- & $-\quad 0.49$ \\
\hline 16 & 0 & 4.63 & -. & -0.628 \\
\hline 23 & 2 & -1.18 & $\cdots$ & $\begin{array}{r}- \\
-\end{array}$ \\
\hline 32 & 0 & 3.92 & $\cdots$ & 0.61 \\
\hline 38 & 3 & 0.55 & - & -* 0.524 \\
\hline 42 & 4 & -0.20 & 0.505 & $--\quad--$ \\
\hline 46 & 4 & 0.47 & - & --0.522 \\
\hline 64 & 3 & 0.63 & - & -0.526 \\
\hline 70 & 3 & -0.78 & - & -- 0.49 \\
\hline 85 & 3 & -0.67 & - & - 0.493 \\
\hline 86 & 4 & -0.16 & 0.506 & $-\infty \quad-$ \\
\hline 89 & 4 & -0.04 & - & - 0.509 \\
\hline 102 & 4 & 0.35 & $\cdots$ & -0.519 \\
\hline 105 & 1 & -1.80 & - & $=0.464$ \\
\hline 113 & 4 & -0.12 & - & -0.507 \\
\hline 134 & 3 & 0.71 & - & -0.528 \\
\hline 138 & 3 & 0.59 & - & -0.525 \\
\hline 142 & 4 & 0.51 & $\cdots$ & -0.523 \\
\hline 149 & 4 & 0.43 & - & $=0.521$ \\
\hline 180 & 3 & -0.55 & - & -0.496 \\
\hline 183 & 3 & 0.71 & - & --0.528 \\
\hline 190 & 4 & -0.20 & - & -0.505 \\
\hline 212 & 0 & -2.35 & $=$ & $\begin{array}{l}-\quad 0.45 \\
\end{array}$ \\
\hline 220 & 4 & 0.12 & - & -0.513 \\
\hline 224 & 2 & -1.02 & - & --0.484 \\
\hline 227 & 4 & -0.27 & 0.503 & $\begin{array}{lll}- & -\end{array}$ \\
\hline 234 & 1 & 1.57 & -. & $-\quad 0.55$ \\
\hline 247 & 4 & 0.00 & - & $=\quad 0.51$ \\
\hline 256 & 0 & 2.35 & - & $--\quad 0.57$ \\
\hline 259 & 4 & -0.16 & - & -0.506 \\
\hline 321 & 0 & -2.86 & - & -0.437 \\
\hline 328 & 3 & -0.78 & -- & $=0.49$ \\
\hline 341 & 3 & 0.90 & -- & -0.533 \\
\hline 366 & 3 & -0.67 & - & -0.493 \\
\hline 370 & 4 & 0.39 & - & - $\quad 0.52$ \\
\hline 372 & 0 & 3.14 & - & 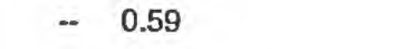 \\
\hline
\end{tabular}


Table 12. Statistical summary of reported data for standard reference sample M-162 (major constituents) -- continued

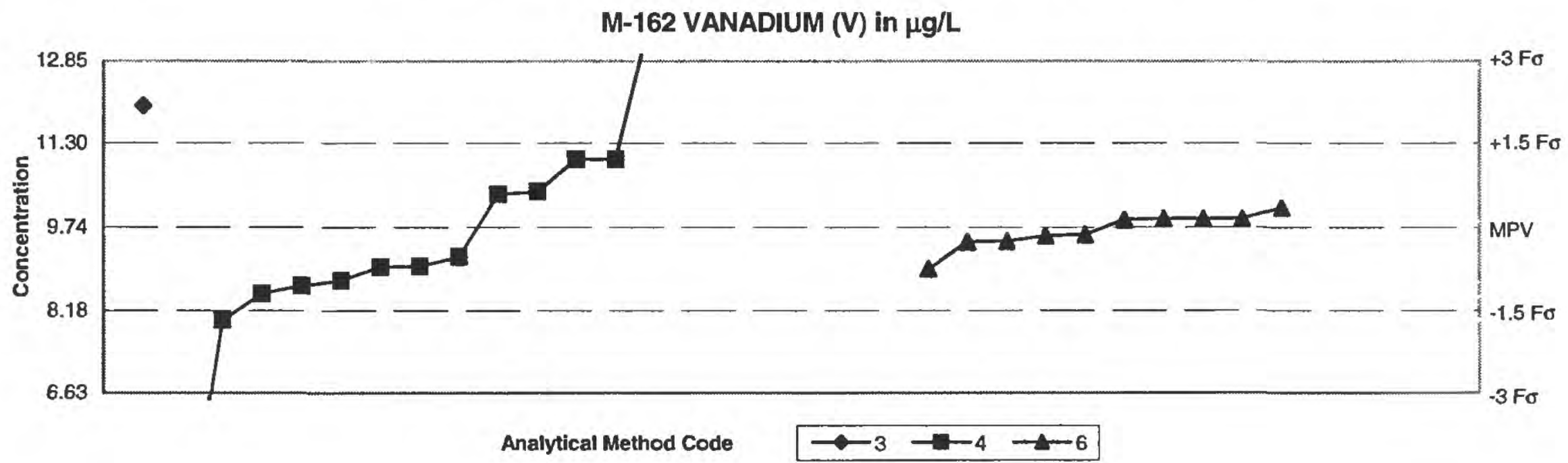

\begin{tabular}{|c|c|c|c|c|c|c|}
\hline \multirow[t]{2}{*}{ SUMMARY } & \multicolumn{4}{|c|}{ Methods } & \multicolumn{2}{|c|}{ Statistics } \\
\hline & 3 & 4 & 6 & Method Codes & & \\
\hline$n=$ & 1 & 15 & 10 & 03 Alomic absorption: graphite fumace & MPV $=$ & $9.74 \mu \mathrm{g} / \mathrm{L}$ \\
\hline Minimum $=$ & 12 & 3.2 & 8.97 & 04 Inductively coupled plasma & F-pseudosigma $=$ & 1.04 \\
\hline Median $=$ & & 9.19 & 9.74 & & $\mathrm{Uh}=$ & 10.40 \\
\hline F-pseudosigma $=$ & & 1.70 & 0.31 & & $\operatorname{Ln}=$ & 9.00 \\
\hline
\end{tabular}

\begin{tabular}{|c|c|c|c|c|c|}
\hline \multirow[b]{2}{*}{ Lab } & \multirow[b]{2}{*}{ Rating } & \multirow[b]{2}{*}{ Z-value } & \multicolumn{3}{|r|}{ Method Codes } \\
\hline & & & 3 & 4 & 6 \\
\hline 1 & 3 & 0.60 & $\overline{--}$ & 10.36 & - \\
\hline 5 & 0 & -5.53 & - & $<4.00$ & - \\
\hline 8 & 4 & 0.15 & -- & - & 9.9 \\
\hline 16 & 2 & 1.21 & - & 11 & - \\
\hline 25 & NR & - & - & $<19$ & - \\
\hline 32 & 4 & 0.15 & 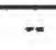 & -. & 9.9 \\
\hline 42 & 4 & -0.13 & -- & $\cdots$ & 9.6 \\
\hline 45 & 4 & -0.26 & -- & -. & 9.47 \\
\hline 59 & 3 & -0.74 & - & - & 8.97 \\
\hline 76 & 4 & 0.13 & - & - & 9.88 \\
\hline 85 & 0 & 5.07 & - & 15 & - \\
\hline 86 & 2 & -1.19 & -. & 8.5 & - \\
\hline 89 & 0 & 2.18 & 12 & - & - \\
\hline 97 & NR & -. & - & $<7.8$ & - \\
\hline 102 & 0 & 4.10 & - & 14 & - \\
\hline 105 & $\overline{N R}$ & -- & - & $<20.0$ & $=$ \\
\hline 121 & 2 & 1.21 & - & 11 & - \\
\hline 134 & 3 & -0.53 & - & 9.19 & - \\
\hline 138 & 3 & -0.95 & - & 8.75 & -- \\
\hline 142 & 4 & -0.25 & - & - & 9.48 \\
\hline 146 & 3 & -0.69 & - & 9.02 & - \\
\hline 180 & 4 & -0.15 & -- & -- & 9.58 \\
\hline 234 & 3 & 0.64 & -- & 10.4 & -- \\
\hline 245 & 4 & 0.35 & - & - & 10.1 \\
\hline 256 & 2 & -1.05 & - & 8.65 & - \\
\hline 265 & 0 & 4.10 & - & 14 & - \\
\hline 305 & 0 & -6.30 & - & 3.2 & .- \\
\hline 328 & 1 & -1.68 & - & 8 & -- \\
\hline 341 & 4 & 0.15 & - & -- & 9.9 \\
\hline 372 & 3 & -0.71 & - & 9 & - \\
\hline
\end{tabular}


Table 13. Statistical summary of reported data for standard reference sample $\mathrm{N}-73$ (nutrient constituents)

N-73 AMMONIA as NITROGEN $\left(\mathrm{NH}_{3}\right.$ as $\left.\mathrm{N}\right)$ in $\mathrm{mg} / \mathrm{L}$

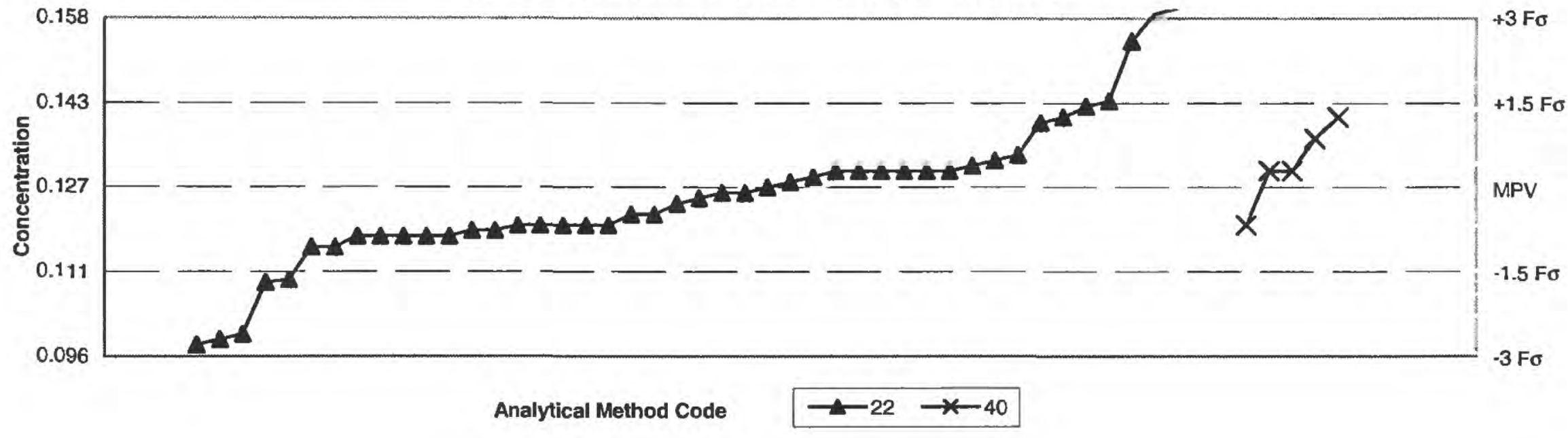

\begin{tabular}{|c|c|c|c|c|c|c|c|}
\hline SUMMARY & & & & & & Stati: & fics \\
\hline & 7 & 20 & 22 & 40 & Method Codes & & \\
\hline$n=$ & $\overline{1}$ & 1 & 46 & 5 & 07 lon chromatography & MPV = & $0.127 \mathrm{mg} / \mathrm{L}$ \\
\hline Minimum $=$ & 0.174 & 0.17 & 0.098 & 0.12 & 20 Titration: colonimetric & F-pseudosigma $=$ & 0.010 \\
\hline Maximum $=$ & & & 0.2 & 0.14 & 22 Colorimetric & $n=$ & 53 \\
\hline Median $=$ & & & 0.126 & 0.130 & 40 Ion selective electrode & $U \mathrm{n}=$ & 0.133 \\
\hline F-pseudosigma $=$ & & & 0.010 & 0.004 & & $\operatorname{Lh}=$ & 0.119 \\
\hline
\end{tabular}

\begin{tabular}{|c|c|c|c|c|c|c|c|c|c|c|c|}
\hline \multirow[b]{2}{*}{ Lab } & \multirow[b]{2}{*}{ Rating } & \multicolumn{4}{|c|}{ Method Codes } & \multirow[b]{2}{*}{ Lab } & \multirow[b]{2}{*}{ Rating } & \multirow[b]{2}{*}{$Z$-value } & \multicolumn{3}{|c|}{ Method Codes } \\
\hline & & Z-value & 7 & 22 & 40 & & & & 7 & 20 & 40 \\
\hline 1 & 3 & -0.77 & $\overline{-}$ & -0.119 & - & 313 & 3 & -0.77 & - & -0.119 & - \\
\hline 5 & 0 & 2.60 & - & - 0.154 & -- & 316 & 1 & -1.69 & .. & -- 0.11 & .. \\
\hline 8 & 0 & 4.14 & - & $0.17 \quad$ & - & 318 & 4 & -0.19 & .. & -0.125 & -- \\
\hline 10 & 4 & 0.29 & - & - & 0.13 & 320 & 4 & 0.29 & -. & -0.13 & -- \\
\hline 12 & 3 & -0.67 & - & 0.12 & - & 321 & 4 & 0.29 & -- & --0.13 & -- \\
\hline 16 & 3 & -0.67 & - & -0.12 & $\ldots$ & 328 & 1 & -1.64 & - & 0.11 & -- \\
\hline 21 & 3 & -0.87 & -- & -0.118 & - & 333 & 0 & 4.53 & 0.174 & -- & -. \\
\hline 23 & 0 & -2.79 & -- & -0.098 & - & 341 & 4 & -0.48 & -- & .- 0.122 & -. \\
\hline 25 & 4 & 0.29 & -. & .. 0.13 & -- & 366 & 4 & 0.29 & -. & -0.13 & -- \\
\hline 26 & NR & - & $<0,4$ & -- $\quad--$ & -- & 370 & 0 & 7.03 & - & $-\quad 0.2$ & -. \\
\hline 31 & 4 & -0.29 & - & -0.124 & -- & 372 & 1 & 1.54 & - & -0.143 & $\overline{-}$ \\
\hline 33 & 4 & 0.48 & -. & -0.132 & -- & 373 & 4 & 0.10 & -. & -0.128 & -- \\
\hline 38 & 3 & 0.58 & - & - 0.133 & .- & 376 & 0 & 3.08 & -. & -0.159 & -- \\
\hline 45 & 3 & 0.87 & -- & -- $\quad$-- & 0.136 & 377 & 3 & -0.67 & .- & $\begin{array}{ll}- & 0.12\end{array}$ & -- \\
\hline 46 & 2 & -1.06 & - & -0.116 & - & & & & & & \\
\hline 51 & 4 & 0.29 & - & $--\quad \quad-$ & 0.13 & & & & & & \\
\hline 59 & 3 & -0.67 & - & 0.12 & - & & & & & & \\
\hline 64 & 2 & 1.25 & - & - $\quad 0.14$ & - & & & & & & \\
\hline 70 & 3 & -0.87 & - & -0.118 & - & & & & & & \\
\hline 72 & 0 & -2.60 & - &.$\quad 0.1$ & - & & & & & & \\
\hline 85 & 4 & 0.29 & - & --0.13 & - & & & & & & \\
\hline 86 & 4 & 0.19 & - & - 0.129 & -- & & & & & & \\
\hline 89 & 3 & -0.87 & - & .- 0.118 & -. & & & & & & \\
\hline 102 & 0 & 3.18 & - & $-\quad 0.16$ & -- & & & & & & \\
\hline 105 & 3 & -0.67 & - & $\begin{array}{ll}-- & 0.12 \\
\end{array}$ & - & & & & & & \\
\hline 110 & 2 & 1.16 & -- & -0.139 & - & & & & & & \\
\hline 113 & 0 & -2.70 & - & -0.099 & - & & & & & & \\
\hline 118 & 3 & -0.87 & - & -0.118 & - & & & & & & \\
\hline 134 & 4 & -0.10 & - & --0.126 & - & & & & & & \\
\hline 138 & 4 & -0.48 & -. & -0.122 & -- & & & & & & \\
\hline 142 & 4 & -0.10 & - & --0.126 & - & & & & & & \\
\hline 146 & 2 & 1.45 & -- & - 0.142 & -- & & & & & & \\
\hline 180 & 4 & 0.00 & - & -0.127 & -. & & & & & & \\
\hline 190 & 3 & -0.87 & - & -0.118 & - & & & & & & \\
\hline 193 & 2 & -1.06 & - & -0.116 & - & & & & & & \\
\hline 198 & 4 & 0.39 & - & -0.131 & - & & & & & & \\
\hline 227 & 0 & 4.34 & .- & $=0.172$ & .. & & & & & & \\
\hline 234 & 3 & -0.67 & - & -- - - & 0.12 & & & & & & \\
\hline 247 & 4 & 0.29 & -- & $=0.13$ & -- & & & & & & \\
\hline 306 & 2 & 1.25 & -- & $\begin{array}{ll}- & -\end{array}$ & 0.14 & & & & & & \\
\hline
\end{tabular}


Table 13. Statistical summary of reported data for standard reference sample $\mathrm{N}-73$ (nutrient constituents) -- continued

N-73 AMMONIA + ORGANIC NITROGEN as NITROGEN $\left(\mathrm{NH}_{3}+\right.$ Organic $\mathrm{N}$ as $\left.\mathrm{N}\right)$ in $\mathrm{mg} / \mathrm{L}$

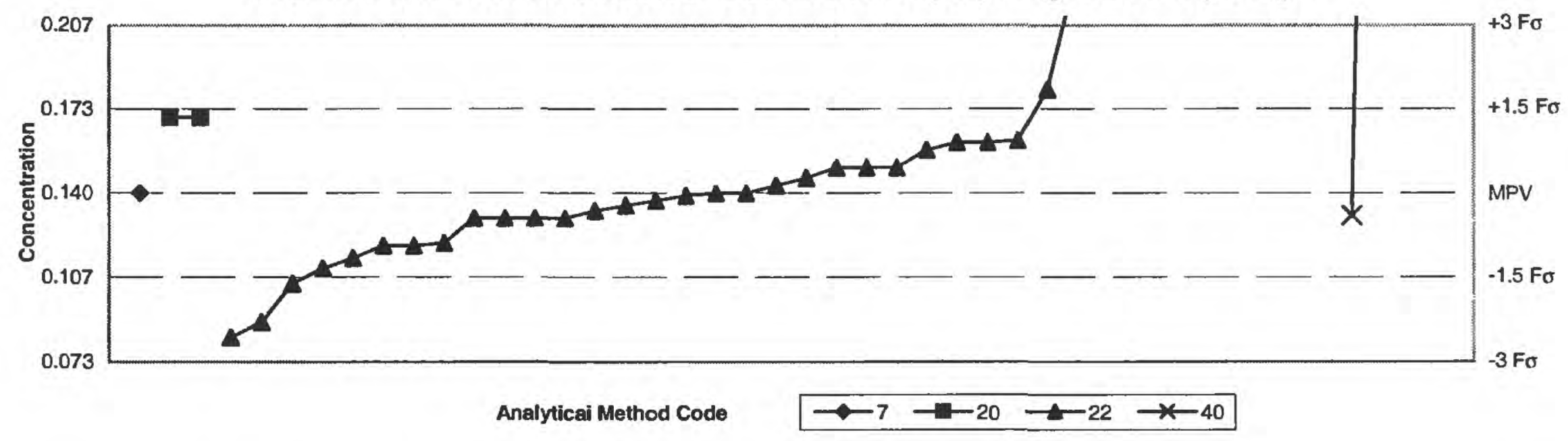

\begin{tabular}{|c|c|c|c|c|c|}
\hline \multirow[t]{2}{*}{ SUMMARY } & \multicolumn{5}{|c|}{ Methods } \\
\hline & 7 & 20 & 22 & 40 & Method Codes \\
\hline $\mathrm{n}=$ & 1 & 2 & $\overline{31}$ & 2 & 07 Ion chromatography \\
\hline Minimum $=$ & 0.14 & 0.17 & 0.083 & 0.131 & 20 Titration: colorimetric \\
\hline Maximum $=$ & & 0.17 & 0.308 & 0.651 & 22 Colorimetric \\
\hline Median $=$ & & & 0.139 & & 40 Ion selective electrode \\
\hline F-pseudosigma $=$ & & & 0.021 & & \\
\hline
\end{tabular}

$\begin{aligned} & \text { Statistics } \\ & \text { MPV }= 0.140 \mathrm{mg} / \mathrm{L} \\ & \text { F-pseudosigma }=0.022 \\ & \mathrm{n}= 36 \\ & \mathrm{Uh}= 0.160 \\ & \text { Lh }=0.130\end{aligned}$

F-pseudosigma $=$

0.021

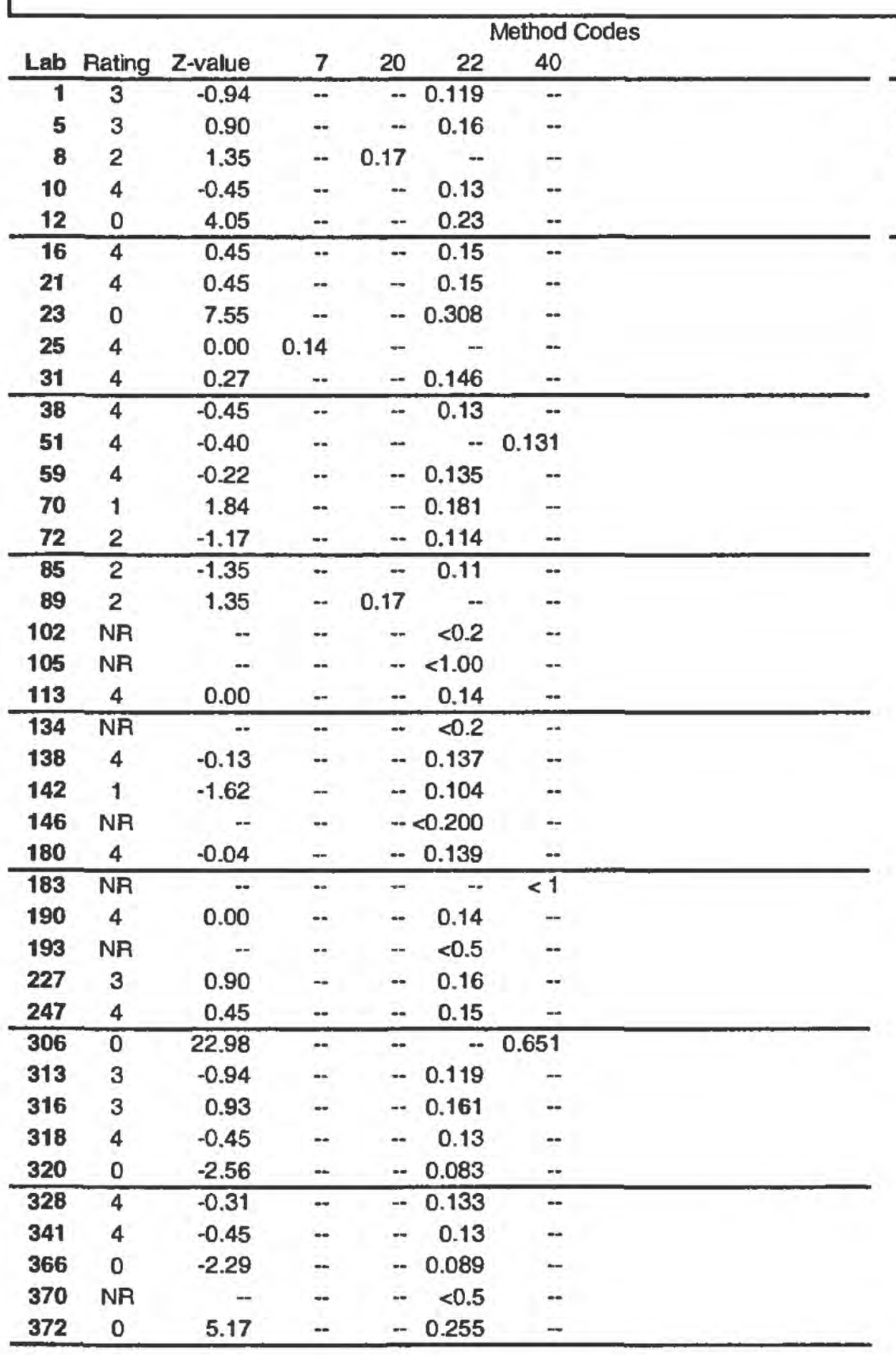


Table 13. Statistical summary of reported data for standard reference sample $\mathrm{N}-73$ (nutrient constituents) -- continued

N-73 NITRATE as NITROGEN $\left(\mathrm{NO}_{3}\right.$ as $\left.\mathrm{N}\right)$ in $\mathrm{mg} / \mathrm{L}$

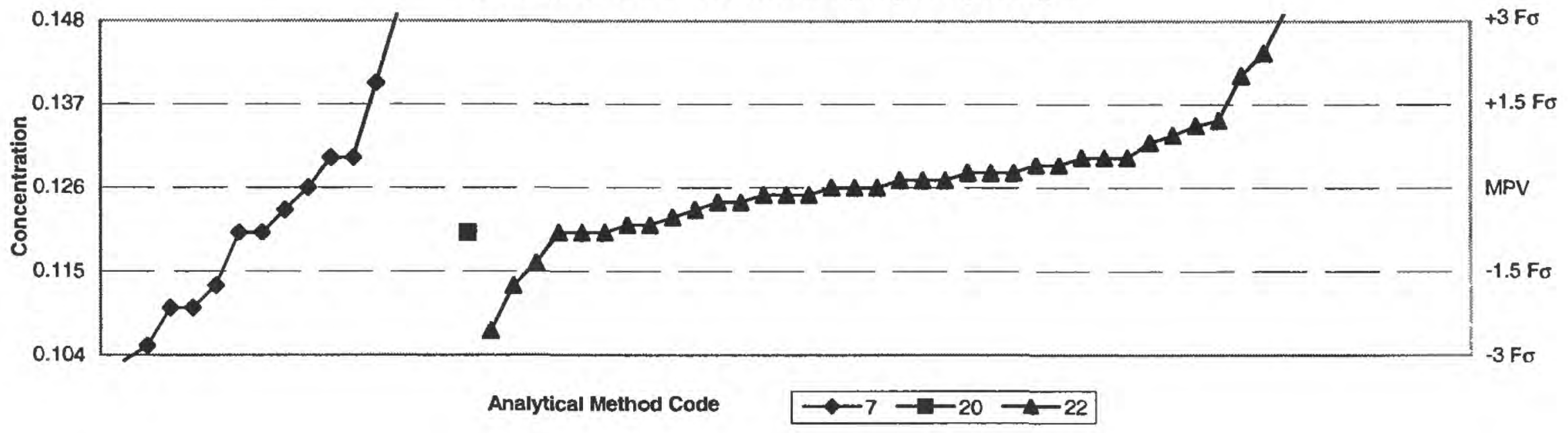

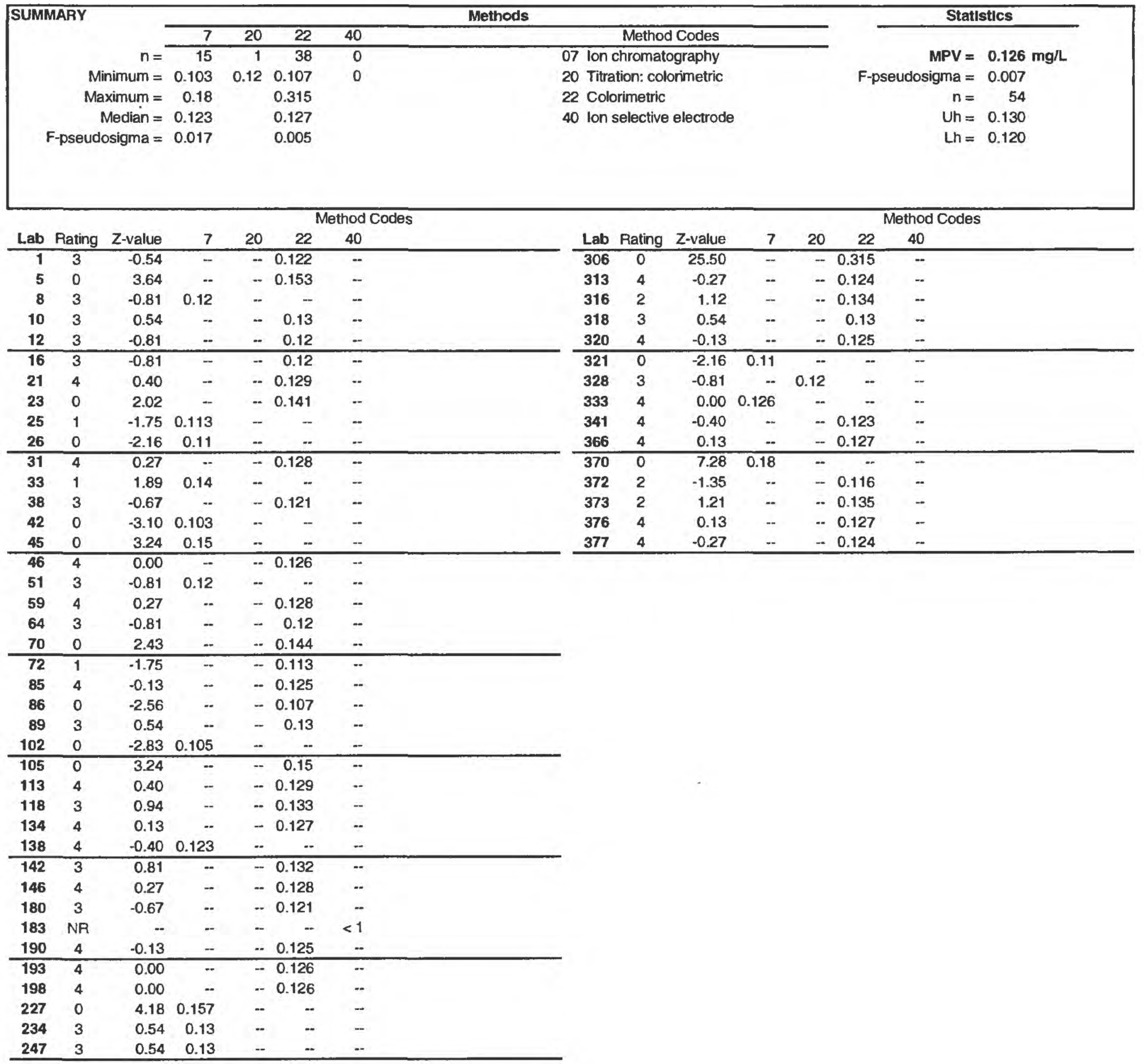


Table 13. Statistical summary of reported data for standard reference sample $\mathrm{N}-73$ (nutrlent constituents) -- continued

N-73 TOTAL PHOSPHORUS as PHOSPHORUS (Total P as P) in mg/L

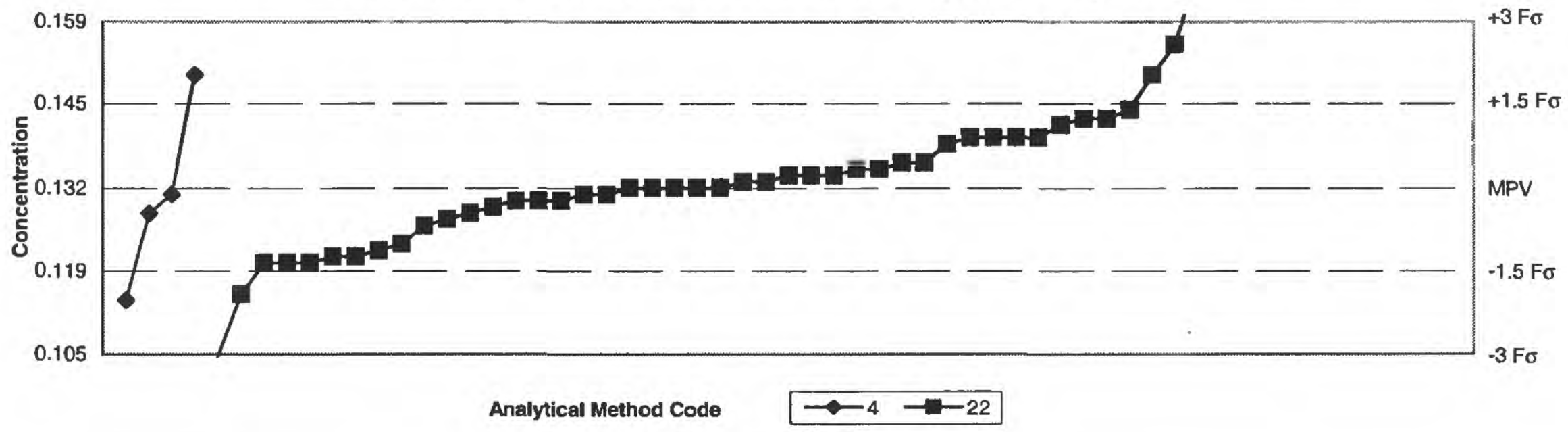

\begin{tabular}{|c|c|c|c|}
\hline \multirow[t]{2}{*}{ SUMMARY } & \multicolumn{2}{|c|}{ Methods } & \multirow[t]{2}{*}{ Statistics } \\
\hline & $4 \quad 22$ & Method Codes & \\
\hline$n=$ & $4 \quad 47$ & 04 Inductively coupled plasma & $M P V=0$. \\
\hline Minimum $=$ & $0.114 \quad 0.105$ & 22 Colorimetric & F-pseudosigma $=0.00$ \\
\hline Maximum $=$ & $0.15 \quad 0.244$ & & $n=$ \\
\hline Median $=$ & 0.133 & & $U h=0.1$ \\
\hline F-pseudosigma $=$ & 0.009 & & $\mathrm{Lh}=0.12$ \\
\hline
\end{tabular}

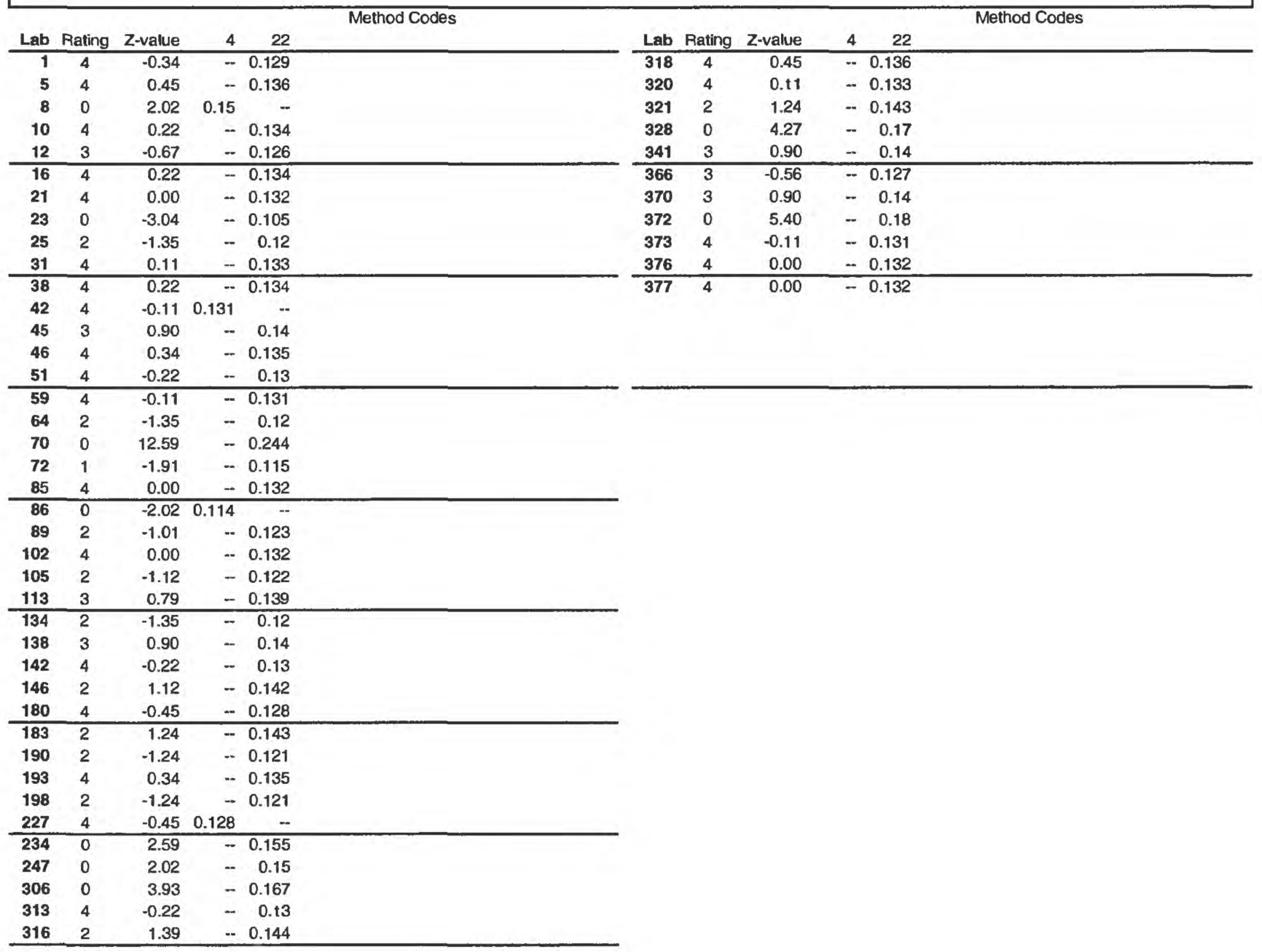


Table 13. Statistical summary of reported data for standard reference sample $\mathbf{N}-73$ (nutrient constituents) -- continued $\mathrm{N}-73$ ORTHOPHOSPHATE as PHOSPHORUS $\left(\mathrm{PO}_{4}\right.$ as $\left.\mathrm{P}\right)$ in $\mathrm{mg} / \mathrm{L}$

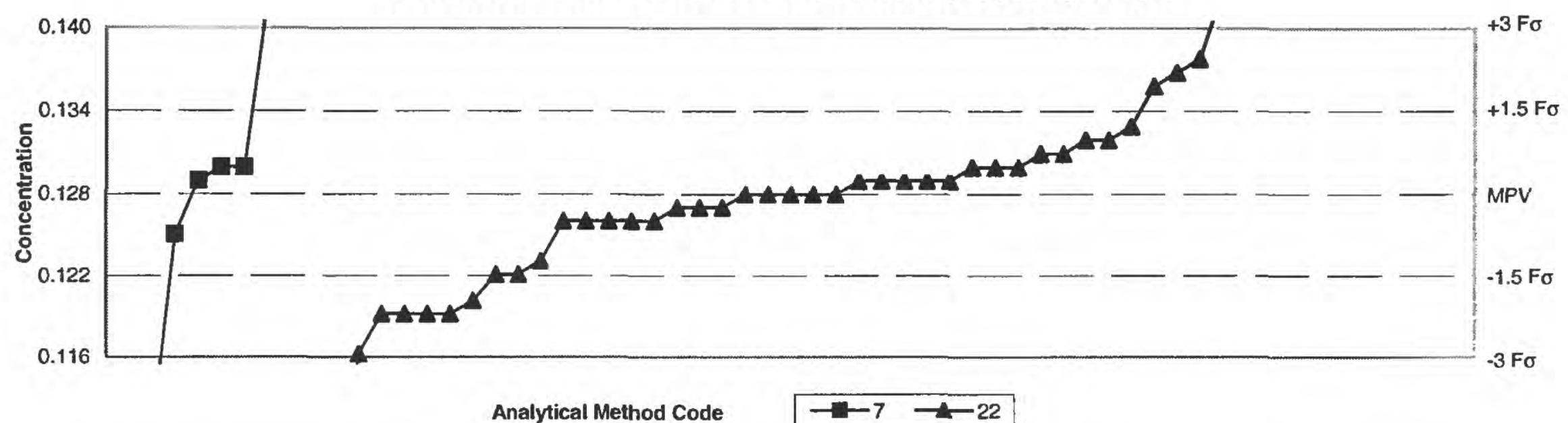

\begin{tabular}{|c|c|c|c|}
\hline \multirow[t]{2}{*}{ SUMMARY } & \multicolumn{3}{|c|}{ Methods } \\
\hline & 0 & 22 & Method Codes \\
\hline $\mathrm{n}=$ & 1 & 43 & 00 Other \\
\hline Minimum $=$ & 0.52 & 0.110 .099 & 07 Ion chromatography \\
\hline Maximum = & & 0.150 .152 & 22 Colorimetric \\
\hline Median $=$ & & 0.1300 .128 & \\
\hline F-pseudosigma = & & $0.007 \quad 0.004$ & \\
\hline
\end{tabular}

\begin{tabular}{rr} 
Statlstlcs \\
\hline MPV $=$ & $0.128 \mathrm{mg} / \mathrm{L}$ \\
F-pseudosigma & $=0.004$ \\
Rating criterion & $=0.006$ \\
$n=$ & 51 \\
Un & $=0.131$ \\
Lh & $=0.126$
\end{tabular}

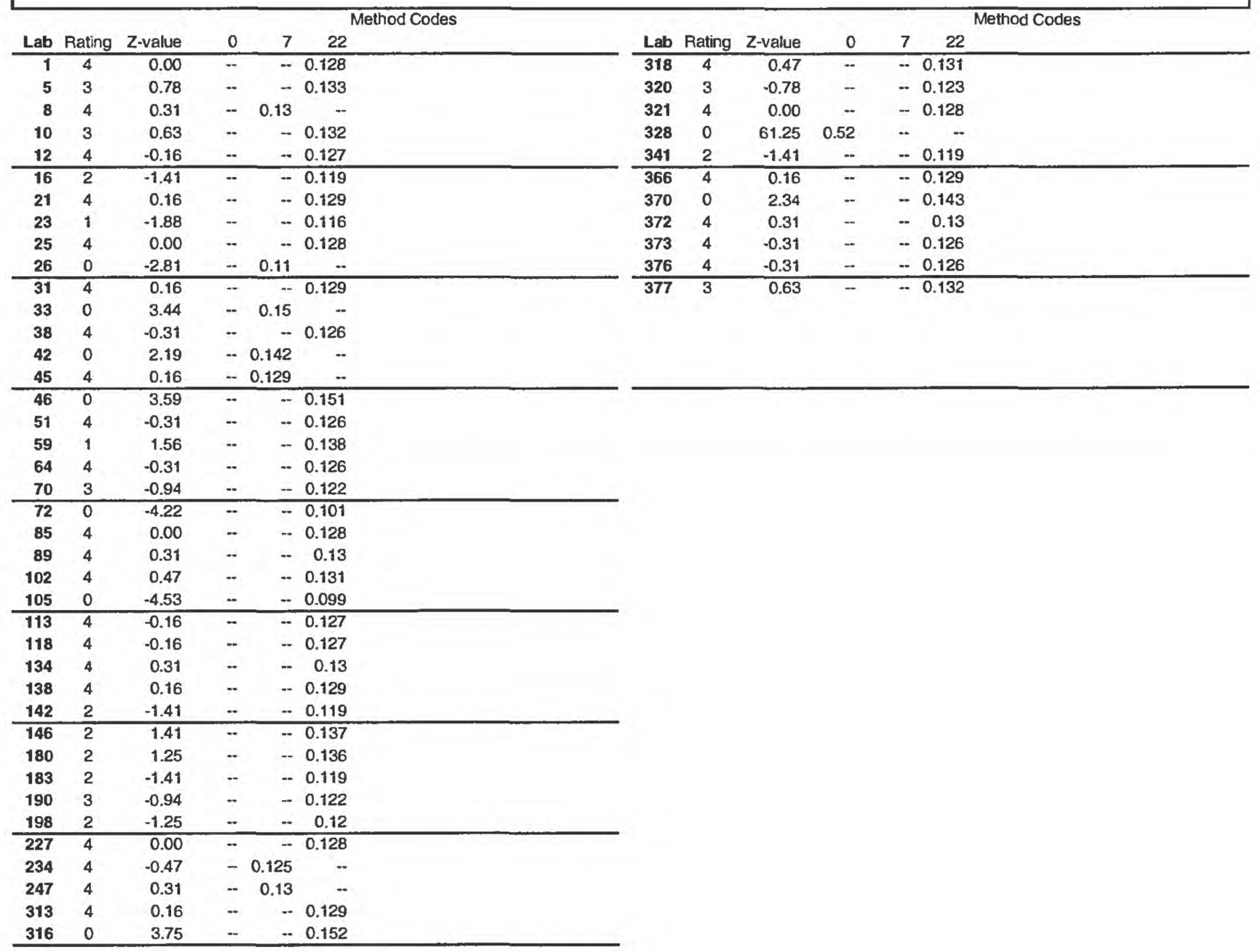


Table 14. Statistical summary of reported data for standard reference sample $\mathbf{N}-74$ (nutrient constituents)

N-74 AMMONIA as NITROGEN $\left(\mathrm{NH}_{3}\right.$ as $\left.\mathrm{N}\right)$ in $\mathrm{mg} / \mathrm{L}$

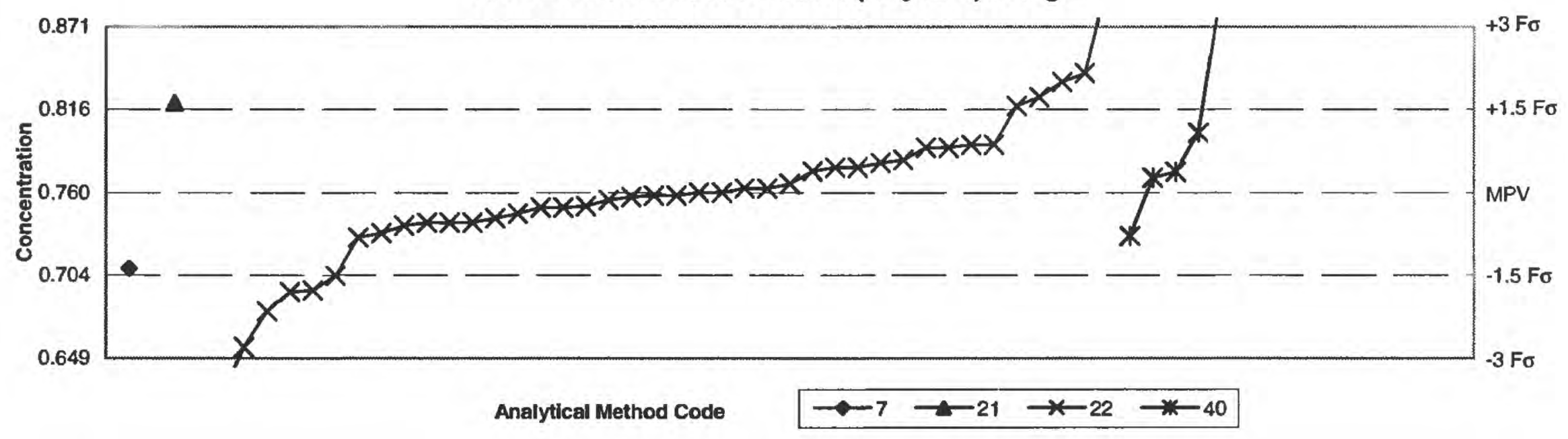

\begin{tabular}{|c|c|c|c|c|c|c|}
\hline \multirow[t]{2}{*}{ SUMMARY } & \multicolumn{6}{|c|}{ Methods } \\
\hline & 7 & 20 & 21 & 22 & 40 & Method Codes \\
\hline$n=$ & 1 & 1 & 1 & 41 & 5 & 07 lon chromatography \\
\hline Minimum $=$ & 0.709 & 1 & 0.82 & 0.574 & 0.731 & 20 Titration: colorimetric \\
\hline Maximum $=$ & & & & 0.9 & 0.9 & 21 Titration: electrometric \\
\hline Median $=$ & & & & 0.758 & 0.774 & 22 Colorimetric \\
\hline F-pseudosigma $=$ & & & & 0.030 & 0.022 & 40 ion selective electrode \\
\hline
\end{tabular}

\begin{tabular}{rr} 
Statistics \\
\hline MPV $=$ & $0.760 \mathrm{mg} / \mathrm{L}$ \\
F-pseudosigma & $=0.037$ \\
Rating criterion & $=0.038$ \\
$n=$ & 49 \\
Un & $=0.790$ \\
Lh & $=0.740$
\end{tabular}

\begin{tabular}{|c|c|c|c|c|c|c|c|c|c|c|c|c|c|c|c|}
\hline \multicolumn{11}{|c|}{ Method Codes } & \multicolumn{5}{|c|}{ Method Codes } \\
\hline Lab & Rating & Z-value & 7 & 20 & 21 & 22 & 40 & Lab & Rating & Z-value & 7 & 20 & 21 & 22 & 40 \\
\hline 1 & 3 & -0.71 & -- & - & $\cdots$ & 0.733 & - & 320 & 3 & 0.84 & -- & -- & - & 0.792 & - \\
\hline 5 & 1 & 1.53 & - & - & - & 0.818 &.- & 328 & 0 & 2.11 & -. & -. & - & 0.84 & - \\
\hline 8 & 0 & 6.32 & -- & 1 & -. & -- & -. & 341 & 4 & 0.16 & - & - & -- & 0.766 & -- \\
\hline 10 & 2 & 1.05 & - & - & -. & - & 0.8 & 356 & 0 & -2.11 & - & - & -- & 0.68 & -- \\
\hline 16 & 3 & -0.53 & -- & - & - & 0.74 & - & 366 & 3 & -0.53 & $\cdots$ & - & - & 0.74 & - \\
\hline 23 & 0 & -2.74 & - & - & $\cdots$ & 0.656 & $\overline{-}$ & 370 & 0 & 3.68 & - & $\overline{--}$ & - & 0.9 & - \\
\hline 25 & 1 & 1.58 & -- & - & 0.82 & - & $\cdots$ & 372 & 4 & .0 .05 & - & -. & -. & 0.758 & - \\
\hline 33 & 4 & -0.08 & - & - & -- & 0.757 & - & 373 & 4 & 0.38 & -- & -- & -- & 0.775 & - \\
\hline 38 & 4 & 0.00 & -. & - & -. & 0.76 & - & 376 & 4 & -0.05 & -- & -- & -- & 0.758 & -. \\
\hline 45 & 2 & -1.34 & 0.709 & - & - & - & 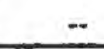 & & & & & & & & \\
\hline 46 & 4 & -0.26 & - & $=$ & $\ldots$ & 0.75 & - & & & & & & & & \\
\hline 59 & 4 & -0.13 & -. & - & -. & 0.755 & - & & & & & & & & \\
\hline 64 & 3 & -0.53 & - & - & - & 0.74 & - & & & & & & & & \\
\hline 70 & 0 & -3.63 & $\ldots$ & -. & - & 0.622 & - & & & & & & & & \\
\hline 72 & 0 & -4.89 & -- & - & $=$ & 0.574 & - & & & & & & & & \\
\hline 80 & 0 & 3,68 & - & - & - & - & 0.9 & & & & & & & & \\
\hline 85 & 3 & 0.58 & -- & -- & -. & 0.782 & -. & & & & & & & & \\
\hline 86 & 1 & -1.76 & $\cdots$ & - & -. & 0.693 & -. & & & & & & & & \\
\hline 89 & 4 & -0.24 & -- & - & - & 0.751 & - & & & & & & & & \\
\hline 97 & 3 & 0.84 & -- & - & - & 0.792 & - & & & & & & & & \\
\hline 102 & 3 & 0.79 & - & - & - & 0.79 & $\cdots$ & & & & & & & & \\
\hline 105 & 4 & 0.00 & - & - & - & 0.76 & - & & & & & & & & \\
\hline 113 & 4 & 0.08 & - & - & -. & 0.763 & - & & & & & & & & \\
\hline 118 & 4 & 0.45 & -. & -- & - & 0.777 & -. & & & & & & & & \\
\hline 134 & 3 & 0.79 & - & - & - & 0.79 & - & & & & & & & & \\
\hline 138 & 3 & -0.58 & - & $=$ & - & 0.738 & - & & & & & & & & \\
\hline 142 & 2 & -1.47 & - & - & - & 0.704 & - & & & & & & & & \\
\hline 146 & 3 & 0.53 & - & - & - & 0.78 & - & & & & & & & & \\
\hline 180 & 4 & 0.08 & -. & - & -. & 0.763 & -- & & & & & & & & \\
\hline 190 & 1 & -1.74 & - & - & - & 0.694 & - & & & & & & & & \\
\hline 193 & 4 & -0.45 & -. & - & -- & 0.743 & -- & & & & & & & & \\
\hline 198 & 1 & 1.68 & - & - & .. & 0.824 & $\cdots$ & & & & & & & & \\
\hline 205 & 4 & 0.45 & -- & -- & -. & 0.777 & -. & & & & & & & & \\
\hline 212 & 3 & -0.79 & $\cdots$ & - & $\cdots$ & 0.73 & - & & & & & & & & \\
\hline 224 & 1 & 1.95 & - & - & $\cdots$ & 0.834 & -. & & & & & & & & \\
\hline 234 & 4 & 0.26 & -- & - & -. & - & 0.77 & & & & & & & & \\
\hline 247 & 4 & -0.26 & - & - & - & 0.75 & -- & & & & & & & & \\
\hline 305 & 4 & 0.37 & - & -- & - & -- & 0.774 & & & & & & & & \\
\hline 306 & 3 & -0.76 & - & .. & - & -- & 0.731 & & & & & & & & \\
\hline 313 & 4 & -0.37 & .. & - & - & 0.746 & -- & & & & & & & & \\
\hline
\end{tabular}


Table 14. Statistical summary of reported data for standard reference sample $\mathrm{N}-74$ (nutrient constituents) - continued

$\mathrm{N}-74$ AMMONIA + ORGANIC NITROGEN as NITROGEN $\left(\mathrm{NH}_{3}+\right.$ Organic $\mathrm{N}$ as $\left.\mathrm{N}\right)$ in $\mathrm{mg} / \mathrm{L}$

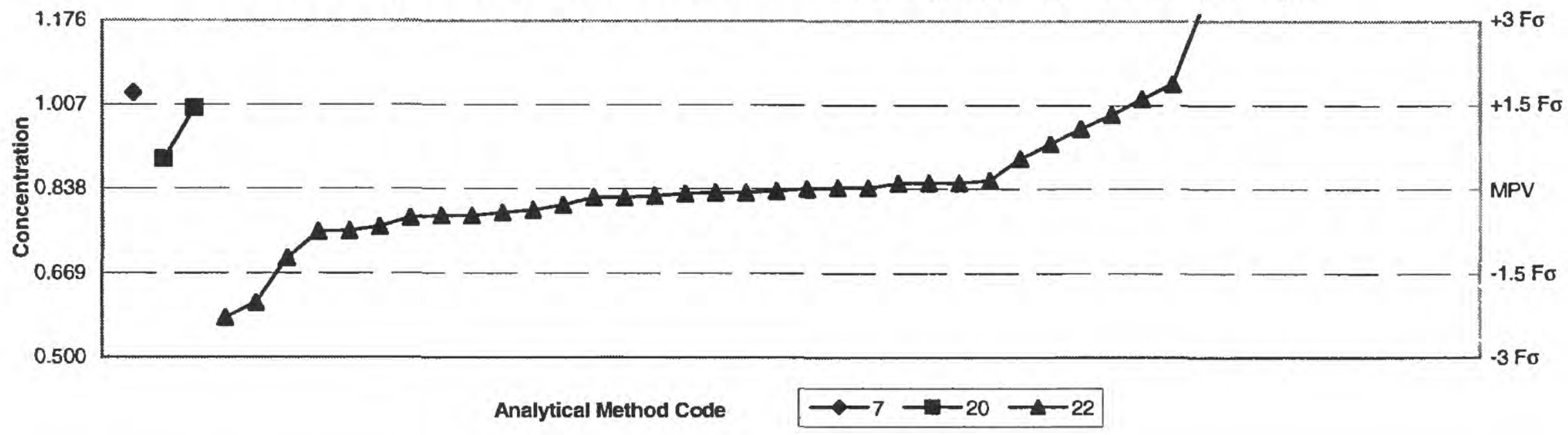

\begin{tabular}{|c|c|c|c|c|c|}
\hline \multirow[t]{2}{*}{ SUMMARY } & \multicolumn{3}{|c|}{ Methods } & \multicolumn{2}{|c|}{ Statistics } \\
\hline & 20 & 22 & Method Codes & & \\
\hline$n=$ & 12 & 36 & 07 Ion chromatography & MPV $=$ & $0.838 \mathrm{mg} / \mathrm{L}$ \\
\hline Minimum = & $1.03 \quad 0.897$ & 0.58 & 20 Titration: colorimetric & F-pseudosigma = & 0.113 \\
\hline Maximum $=$ & 1 & 1.97 & 22 Colorimetric & $n=$ & 39 \\
\hline Median $=$ & & 0.832 & & $U n=$ & 0.945 \\
\hline F-pseudosigma = & & 0.094 & & $\operatorname{Lh}=$ & 0.793 \\
\hline
\end{tabular}

Method Codes

\begin{tabular}{|c|c|c|c|c|c|}
\hline \multirow{2}{*}{ Lab } & \multirow[b]{2}{*}{ Rating } & \multirow[b]{2}{*}{ Z-value } & \multicolumn{3}{|r|}{ Method Codes } \\
\hline & & & 7 & 20 & \\
\hline 1 & 4 & 0.10 & - & $=0.849$ & \\
\hline 5 & 4 & -0.07 & - & -0.83 & \\
\hline 8 & 2 & 1.44 & - & $1 \quad-$ & \\
\hline 10 & 4 & 0.02 & - & $-\quad 0.84$ & \\
\hline 16 & 2 & 1.08 & - & 0.96 & \\
\hline 23 & 1 & 1.88 & $\cdots$ & 1.05 & \\
\hline 25 & 1 & 1.70 & $\mathrm{t} .03$ & $-\quad \quad-$ & \\
\hline 38 & 4 & -0.07 & - & $-\quad 0.83$ & \\
\hline 46 & 2 & 1.33 & - & -0.988 & \\
\hline 59 & 3 & -0.51 & -- & $=\quad 0.78$ & \\
\hline 70 & 4 & 0.00 & - & -0.838 & \\
\hline 72 & 4 & -0.48 & - & -0.784 & \\
\hline 85 & 4 & -0.16 & - & -0.82 & \\
\hline 89 & 3 & 0.52 & - & $0.897 \quad-$ & \\
\hline 97 & 4 & -0.16 & - & $-\quad 0.82$ & \\
\hline 102 & 3 & -0.76 & - & -0.752 & \\
\hline 105 & 1 & 1.62 & -- & $=1.02$ & \\
\hline 113 & 4 & 0.11 & - & $-\quad 0.85$ & \\
\hline 134 & 4 & -0.04 & - & -0.833 & \\
\hline 138 & 4 & 0.11 & - & $=0.85$ & \\
\hline 142 & 4 & 0.15 & - & -0.855 & \\
\hline 146 & 4 & -0.48 & - & $=0.784$ & \\
\hline 180 & 4 & -0.29 & -- & -0.805 & \\
\hline 190 & 3 & -0.75 & - & -0.754 & \\
\hline 193 & 3 & 0.81 & -- & -0.929 & \\
\hline 212 & 0 & -2.29 & -- & - 0.58 & \\
\hline 224 & 3 & -0.67 & - & -0.762 & \\
\hline 247 & 4 & -0.43 & -- & $=0.79$ & \\
\hline 306 & 0 & 5.43 & - & $=1.45$ & \\
\hline 313 & 4 & -0.13 & - & $=0.823$ & \\
\hline 320 & 3 & 0.54 & $\overline{--}$ & $\Rightarrow 0.899$ & \\
\hline 328 & 0 & 6.14 & - & $=1.53$ & \\
\hline 341 & 4 & 0.02 & - & $-\quad 0.84$ & \\
\hline 356 & 4 & -0.38 & - & -0.795 & \\
\hline 366 & 0 & -2.02 & - & $\begin{array}{l}-\quad 0.61 \\
\end{array}$ & \\
\hline 370 & 2 & -1.22 & - & 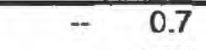 & \\
\hline 372 & 0 & 10.05 & - & - $\quad 1.97$ & \\
\hline 373 & 0 & 3.31 & -. & -1.211 & \\
\hline 376 & 4 & -0.09 & -- & -0.828 & \\
\hline
\end{tabular}


Table 14. Statistical summary of reported data for standard reference sample $\mathbf{N}-74$ (nutrient constituents) -- cont/nued

N-74 NITRATE as NITROGEN $\left(\mathrm{NO}_{3}\right.$ as $\left.\mathrm{N}\right)$ in $\mathrm{mg} / \mathrm{L}$

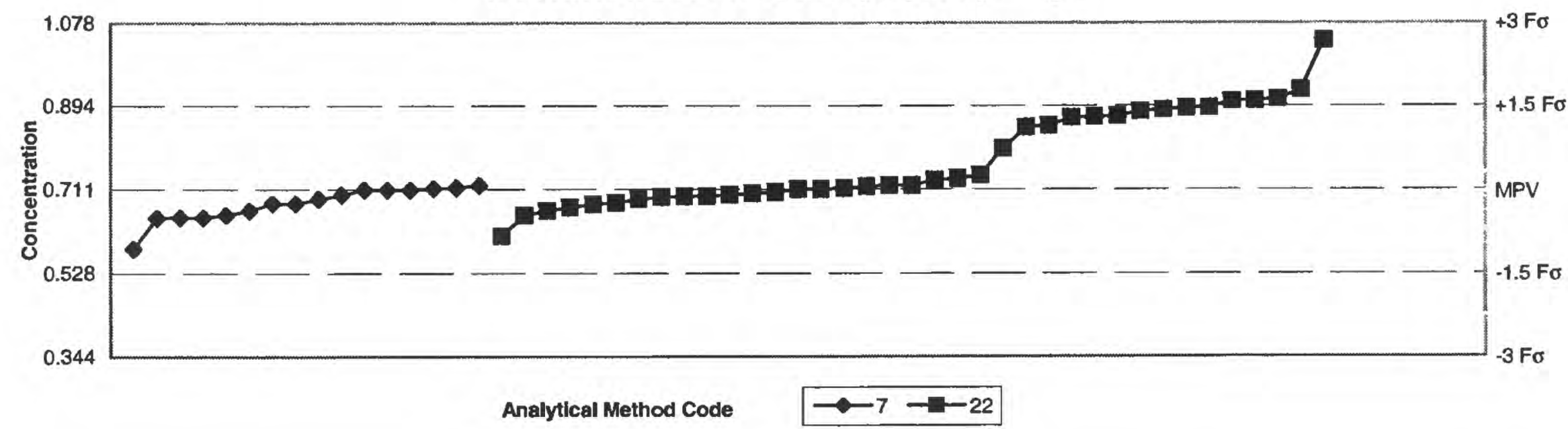

\begin{tabular}{|c|c|c|c|c|c|c|}
\hline \multirow[t]{2}{*}{ SUMMARY } & \multicolumn{4}{|c|}{ Methods } & \multicolumn{2}{|c|}{ Statistics } \\
\hline & 7 & 22 & 40 & Method Codes & & \\
\hline$n=$ & 16 & 37 & 0 & 07 lon chromatography & MPV $=$ & $0.711 \mathrm{mg} / \mathrm{L}$ \\
\hline Minimum $=$ & 0.581 & 0.61 & 0 & 22 Colorimetric & F-pseudosigma $=$ & 0.122 \\
\hline Maximum $=$ & 0.72 & 1.04 & & 40 Ion selective electrode & $n=$ & 53 \\
\hline Median $=$ & 0.685 & 0.720 & & & Un $=$ & 0.847 \\
\hline F-pseudosigma $=$ & 0.043 & 0.129 & & & $\mathrm{Lh}=$ & 0.682 \\
\hline
\end{tabular}

\begin{tabular}{|c|c|c|c|c|c|c|c|c|c|c|c|}
\hline \multicolumn{9}{|c|}{ Method Codes } & \multirow{2}{*}{\multicolumn{3}{|c|}{${ }_{40}$ Method Codes }} \\
\hline Lab & Rating & $Z$-value & 7 & 22 & 40 & Lab & Rating & Z-value & & & \\
\hline 1 & 4 & -0.10 & 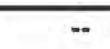 & 0.699 & $\cdots$ & 234 & 4 & -0.51 & 0.649 & -- & -. \\
\hline 5 & 2 & 1.11 & - & 0.847 & -. & 247 & 4 & -0.50 & 0.65 & - & - \\
\hline 8 & 4 & -0.25 & 0.68 & -- & -. & 305 & 4 & -0.01 & 0.71 & -- & - \\
\hline 10 & 2 & 1.30 & -. & 0.87 & $\cdots$ & 306 & 0 & 2.69 & - & 1.04 & - \\
\hline 16 & 3 & -0.83 & - & 0.61 & .. & 313 & 4 & -0.26 & $\cdots$ & 0.679 & - \\
\hline 23 & 2 & 1.46 & - & 0.889 & -- & 320 & 4 & -0.11 & - & 0.697 & - \\
\hline 25 & 4 & -0.46 & 0.655 & -. & $\ldots$ & 328 & 2 & 1.14 & - & 0.85 & - \\
\hline 26 & 4 & -0.50 & 0.65 & -- & - & 341 & 4 & -0.17 & -- & 0.69 & - \\
\hline 30 & 4 & -0.25 & 0.68 & - & -. & 356 & 4 & 0.02 & - & 0.714 & - \\
\hline 33 & 4 & -0.01 & 0.71 & - & -. & 366 & 4 & 0.00 & -- & 0.711 & - \\
\hline 38 & 2 & 1.31 & -- & 0.871 & - & 370 & 4 & 0.03 & 0.715 & -- & $=$ \\
\hline 42 & 2 & -1.06 & 0.581 & -- & -- & 372 & 4 & -0.24 & -- & 0.682 & - \\
\hline 45 & 4 & -0.38 & 0.665 & -- & - & 373 & 4 & -0.05 & -. & 0.705 & - \\
\hline 46 & 4 & 0.25 & -- & 0.742 & -. & 376 & 4 & -0.32 & - & 0.672 & - \\
\hline 59 & 4 & -0.07 & - & 0.702 & -. & & & & & & \\
\hline 64 & 2 & 1.46 & - & 0.89 & - & & & & & & \\
\hline 70 & 4 & -0.14 & -- & 0.694 & - & & & & & & \\
\hline 72 & 4 & 0.07 & .- & 0.72 & -. & & & & & & \\
\hline 80 & 3 & 0.73 & - & 0.8 & - & & & & & & \\
\hline 85 & 4 & 0.07 & .- & 0.72 & -. & & & & & & \\
\hline 86 & 2 & 1.28 & - & 0.868 & -. & & & & & & \\
\hline 89 & 1 & 1.59 & -. & 0.906 & -- & & & & & & \\
\hline 97 & 4 & 0.05 & -. & 0.717 & - & & & & & & \\
\hline 102 & 4 & 0.02 & 0.713 & -. & -- & & & & & & \\
\hline 105 & 1 & 1.79 & -- & 0.93 & $-\cdot$ & & & & & & \\
\hline 113 & 4 & 0.00 & - & 0.711 & $\ldots$ & & & & & & \\
\hline 118 & 1 & 1.59 & -- & 0.905 & - & & & & & & \\
\hline 134 & 1 & 1.63 & - & 0.91 & - & & & & & & \\
\hline 138 & 4 & -0.10 & 0.699 & -. & -- & & & & & & \\
\hline 142 & 2 & 1.42 & -- & 0.885 & - & & & & & & \\
\hline 146 & 4 & -0.47 & - & 0.654 & 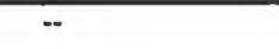 & & & & & & \\
\hline 180 & 4 & -0.38 & - & 0.665 & -- & & & & & & \\
\hline 183 & NR & -- & - & -- & $<1$ & & & & & & \\
\hline 190 & 4 & 0.16 & - & 0.731 & -- & & & & & & \\
\hline 193 & 2 & 1.40 & - & 0.882 & - & & & & & & \\
\hline 198 & 4 & -0.12 & -- & 0.696 & -. & & & & & & \\
\hline 205 & 4 & 0.19 & - & 0.734 & - & & & & & & \\
\hline 208 & 4 & -0.17 & 0.69 & - & $=$ & & & & & & \\
\hline 212 & 4 & -0.01 & 0.71 & -. & - & & & & & & \\
\hline 224 & 4 & 0.07 & 0.72 & -- & - & & & & & & \\
\hline
\end{tabular}


Table 14. Statistical summary of reported data for standard reference sample $\mathrm{N}-74$ (nutrient constituents) -- continued

N-74 TOTAL PHOSPHORUS as PHOSPHORUS (Total P as P) in mg/L

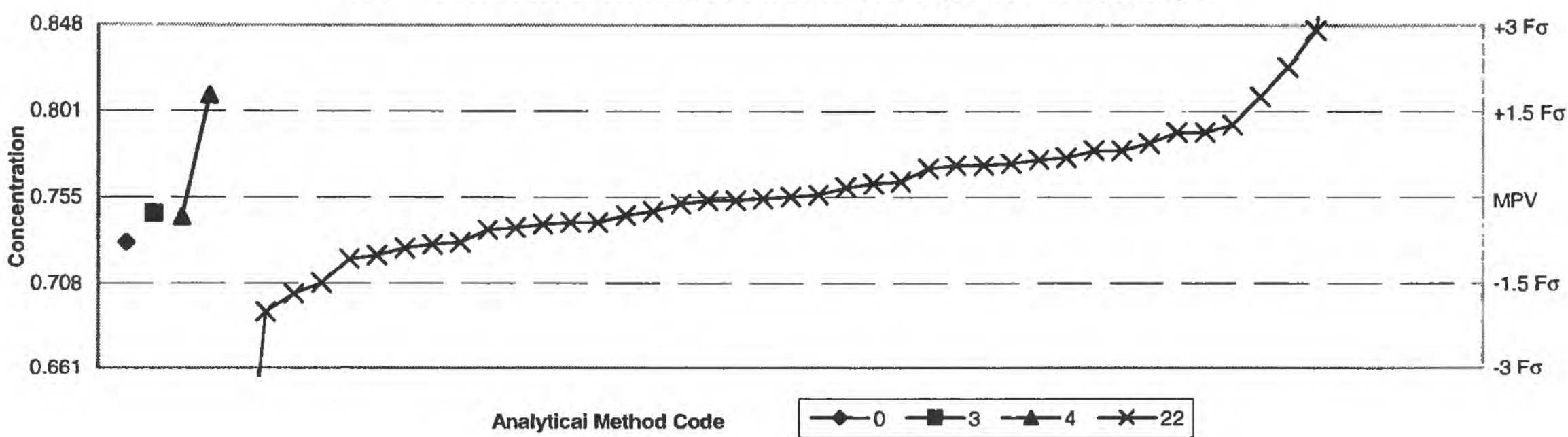

\begin{tabular}{rlrlrlll}
\hline SUMMARY & & & & & & Methods & \multicolumn{1}{c}{ Method Codes } \\
\cline { 2 - 7 }$n$ & $=$ & 1 & 1 & 2 & 42 & O0 Other \\
Minimum & $=$ & 0.73 & 0.746 & 0.744 & 0.536 & 03 Atomic absorption: graphite fumace \\
Maximum & $=$ & & & 0.81 & 1.05 & 04 inductively coupled plasma \\
Median & $=$ & & & & 0.756 & 22 Colorimetric \\
F-pseudosigma & $=$ & & & & 0.031 &
\end{tabular}

$\begin{aligned} & \text { Statistics } \\ & \text { MPV }= 0.755 \mathrm{mg} / \mathrm{L} \\ & \text { F-pseudosigma }=0.031 \\ & \text { Rating criterion }=0.038 \\ & n= 46 \\ & U h= 0.780 \\ & L h= 0.738\end{aligned}$

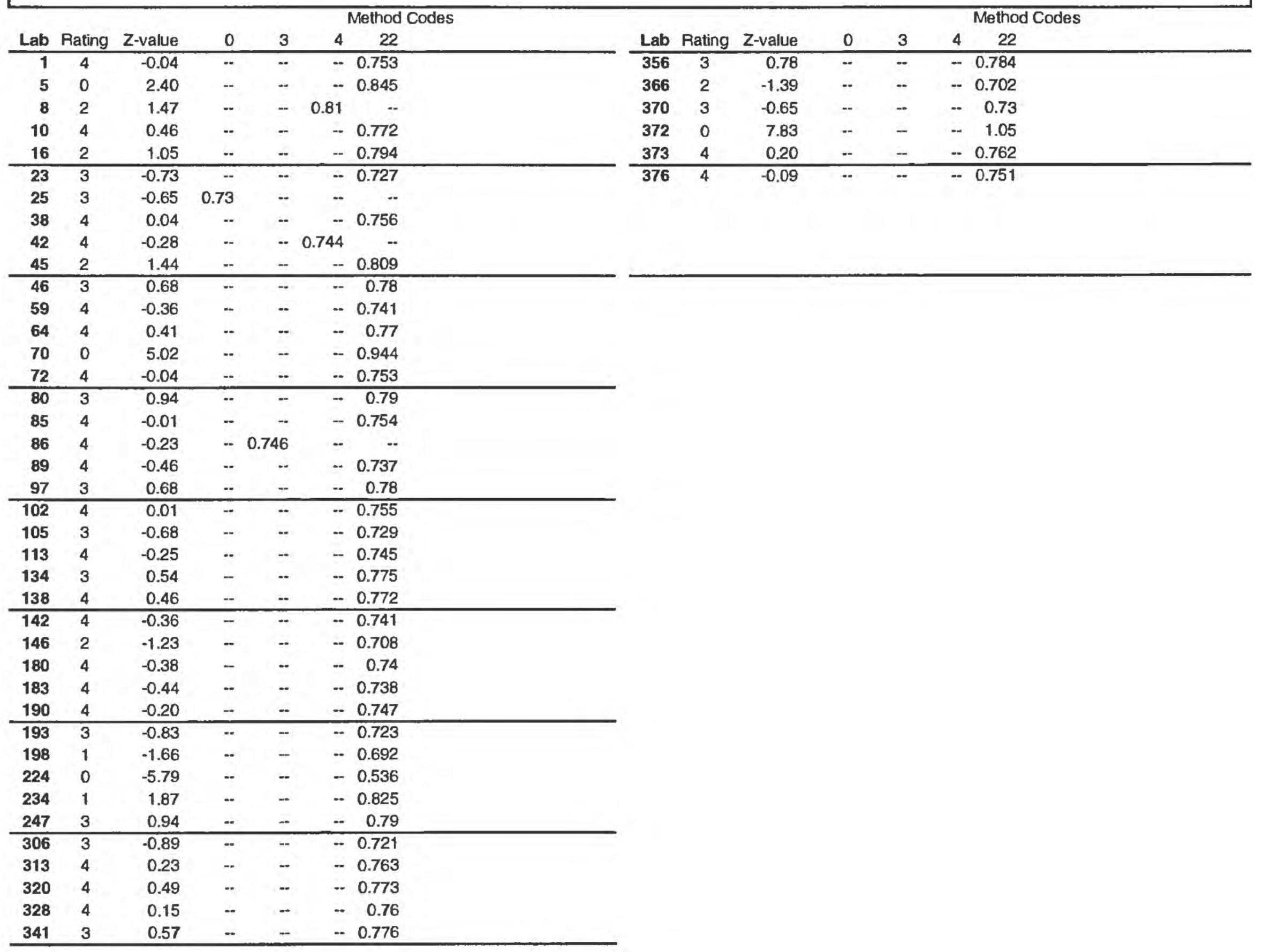


Table 14. Statistical summary of reported data for standard reference sample $\mathbf{N - 7 4}$ (nutrient constituents) -- continued

N-74 ORTHOPHOSPHATE as PHOSPHORUS) in $\mathrm{mg} / \mathrm{L}$

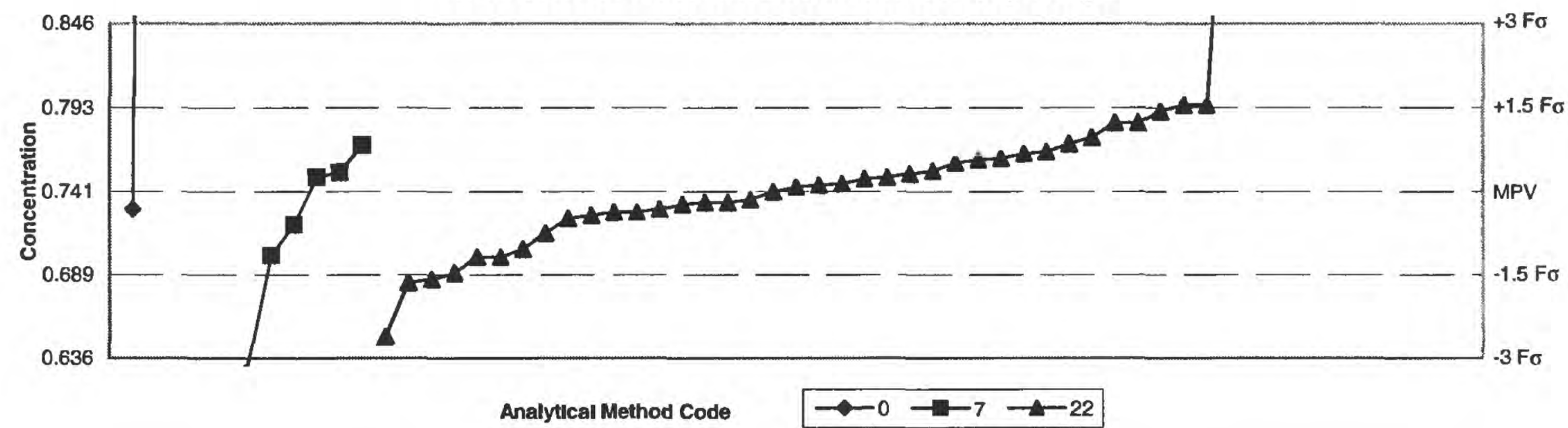

\begin{tabular}{|c|c|c|c|}
\hline \multirow[t]{2}{*}{ SUMMARY } & \multicolumn{3}{|c|}{ Methods } \\
\hline & 0 & 22 & Method Codes \\
\hline$n=$ & 2 & 38 & 00 Other \\
\hline Minimum = & 0.73 & 0.56 & 07 Ion chromatography \\
\hline Maximum $=$ & 2.33 & 0.77 & 22 Colorimetric \\
\hline Median $=$ & & $0.701 \quad 0.745$ & \\
\hline F-pseudosigma = & & 0.0920 .029 & \\
\hline
\end{tabular}

$\begin{aligned} & \text { Statistics } \\ & \text { MPV }=0.741 \mathrm{mg} / \mathrm{L} \\ & \text { F-pseudosigma }=0.035 \\ & \text { Rating criterion }=0.037 \\ & \mathrm{n}= 49 \\ & \text { Uh }=0.762 \\ & \text { Lh }=0.715\end{aligned}$

Method Codes

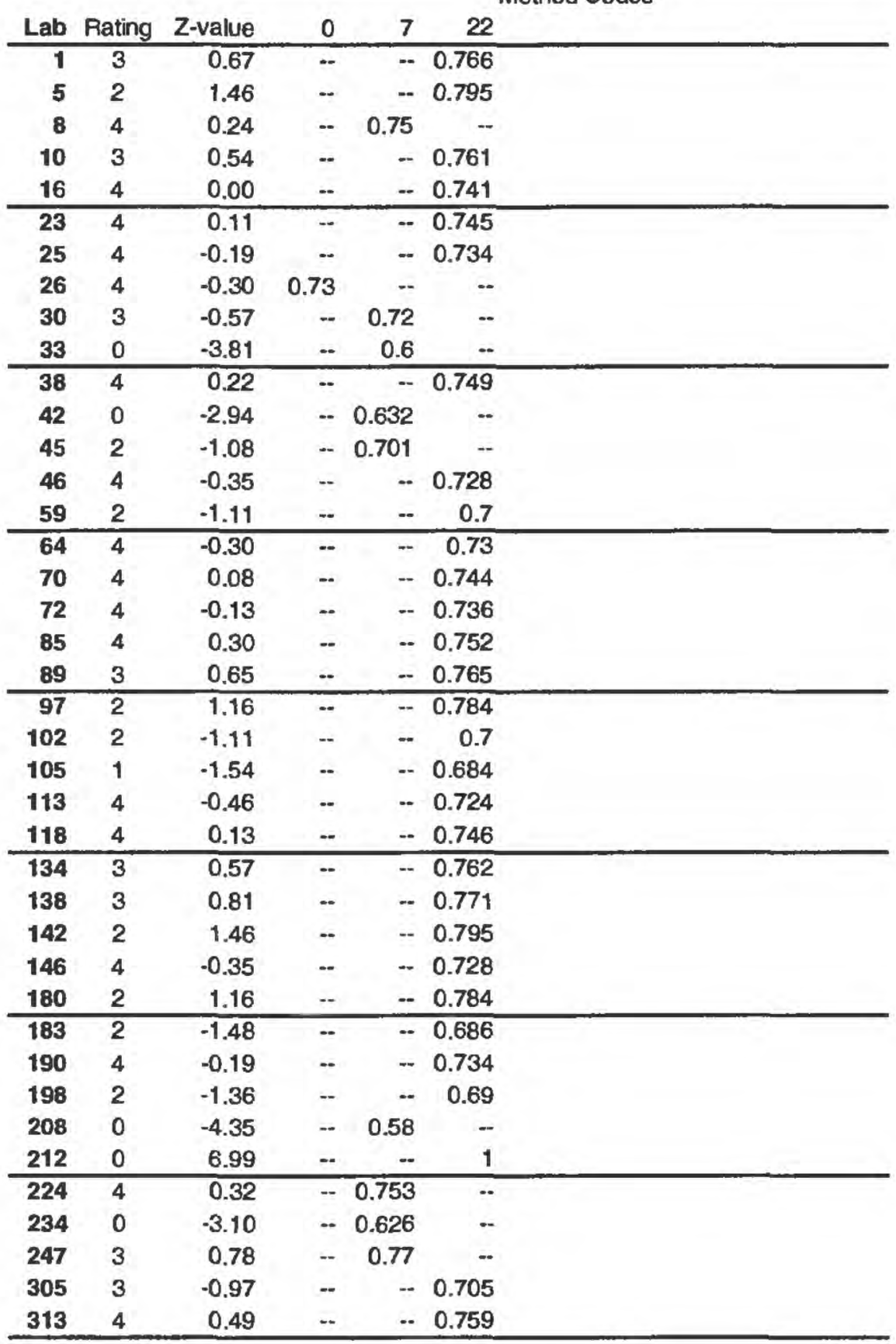

\begin{tabular}{|c|c|c|c|c|c|}
\hline & & & & & Method Codes \\
\hline Lab & Rating & Z-value & 0 & 22 & \\
\hline 320 & 3 & -0.70 & - & $\because 0.715$ & \\
\hline 328 & 0 & 42.89 & 2.33 & -. & \\
\hline 341 & 0 & -2.46 & - & $=0.65$ & \\
\hline 356 & 4 & 0.35 & -. & -0.754 & \\
\hline 366 & 4 & -0.22 & - & -0.733 & \\
\hline 370 & 3 & 0.92 & - & -0.775 & \\
\hline 372 & 4 & 0.24 & -. & $\begin{array}{ll}- & 0.75\end{array}$ & \\
\hline 373 & 4 & -0.40 & - & $\Rightarrow 0.726$ & \\
\hline 376 & 2 & 1.35 & - & $\Rightarrow 0.791$ & \\
\hline
\end{tabular}


Table 15. Statistica/ summary of reported data for standard reference sample P-38 (low ionic strength const/tuents)

P-38 ACIDITY (as $\mathrm{CaCO}_{3}$ ) in $\mathrm{mg} / \mathrm{L}$

\begin{tabular}{|c|c|c|c|c|c|c|}
\hline \multicolumn{3}{|c|}{ SUMMARY } & \multicolumn{3}{|r|}{ Methods } & \multirow{7}{*}{\begin{tabular}{l}
\multicolumn{1}{c}{ Statistics } \\
inadequate data \\
(Fo $>$ MPV)
\end{tabular}} \\
\hline & & & 20 & 21 & Method Codes & \\
\hline & & $\mathrm{n}=$ & 1 & 7 & 20 Titration: colorimetric & \\
\hline & & inimum $=$ & 4.5 & 2.8 & 21 Titration: electrometric & \\
\hline & & ximum $=$ & & 20 & & \\
\hline & & Median $=$ & & 6.80 & & \\
\hline \multicolumn{3}{|c|}{ F-pseudosigma $=$} & & 8.98 & & \\
\hline & & & & & Method Codes & \\
\hline Lab & Rating & $Z$-value & 20 & 21 & & \\
\hline 25 & $\mathrm{NR}$ & 0.88 & -- & 14 & & \\
\hline 38 & NR & -0.32 & - & 2.96 & & \\
\hline 89 & NR & -0.34 & - & 2.8 & & \\
\hline 105 & NR & 0.10 & - & 6.8 & & \\
\hline 247 & NR & -0.10 & -- & 5 & & \\
\hline 328 & NA & -0.15 & 4.5 & - & & \\
\hline 370 & NR & 1.54 & -- & 20 & & \\
\hline 372 & NR & 1.34 & - & 18.2 & & \\
\hline
\end{tabular}


Table 15. Statistical summary of reported data for standard reference sample P-38 (low ionlc strength constituents) - continued

P-38 CALCIUM (Ca) In mg/L

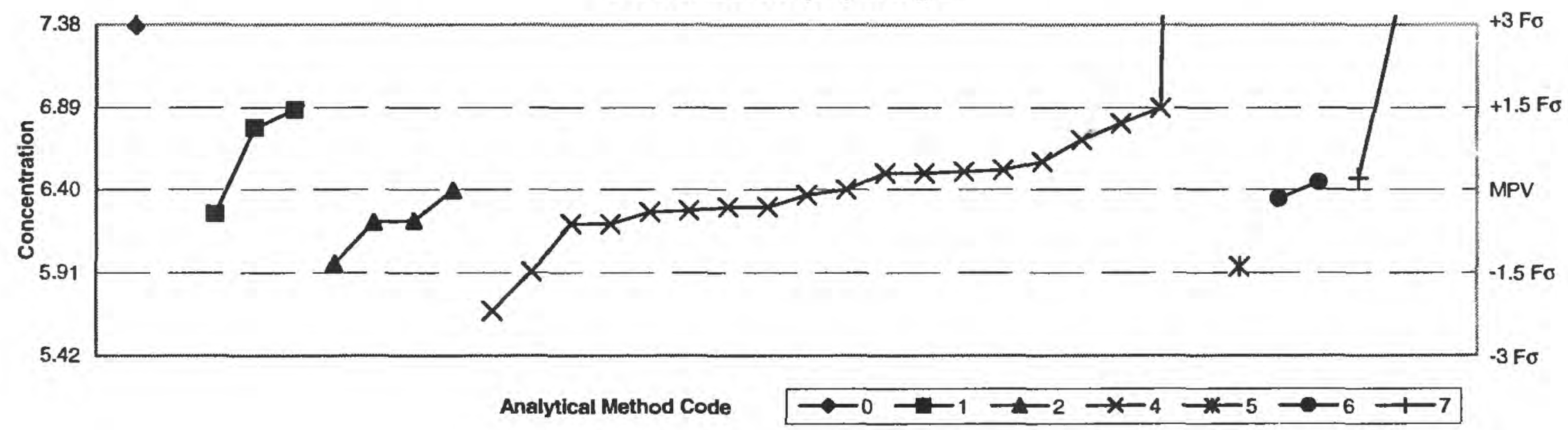

\begin{tabular}{|c|c|c|c|c|c|c|c|c|c|c|}
\hline \multirow[t]{2}{*}{ SUMMARY } & \multicolumn{8}{|c|}{ Methods } & \multicolumn{2}{|c|}{ Statistics } \\
\hline & 0 & 1 & 2 & 4 & 5 & 6 & 7 & Method Codes & & \\
\hline$n=$ & 2 & 3 & 4 & 19 & 1 & 2 & 2 & 00 Other & MPV $=$ & $6.40 \mathrm{mg} / \mathrm{L}$ \\
\hline Minimum $=$ & 7.37 & 6.26 & 5.97 & 5.69 & 5.95 & 6.35 & 6.472 & 01 Atomic absorption: direct, air & F-pseudosigma $=$ & 0.33 \\
\hline Maximum $=$ & 16.12 & 6.88 & 6.4 & 15.7 & & 6.447 & 7.48 & 02 Atomic absorption: direct, nitrous oxide & $n=$ & 33 \\
\hline Median $=$ & & & & 6.40 & & & & 04 Inductively coupled plasma & $\mathrm{Uh}=$ & 6.70 \\
\hline F-pseudosigma $=$ & & & & 0.20 & & & & $\begin{array}{l}05 \text { Direct current plasma } \\
06 \text { Inductively coupled plasma/mass spect } \\
07 \text { Ion chromatography }\end{array}$ & ometry & 6.26 \\
\hline
\end{tabular}

\begin{tabular}{|c|c|c|c|c|c|c|c|c|c|}
\hline \multirow[b]{2}{*}{ Lab } & \multirow[b]{2}{*}{ Rating } & \multirow[b]{2}{*}{ Z-value } & \multicolumn{7}{|c|}{ Method Codes } \\
\hline & & & 0 & 1 & 2 & 4 & 5 & 6 & 7 \\
\hline 1 & 3 & -0.62 & - & - & -- & 6.2 & - & -- & - \\
\hline 2 & 4 & 0.21 & -- & -. & - & -- & - & - & 6.472 \\
\hline 5 & 0 & 28.50 & -- & -- & -- & 15.7 & -- & - & - \\
\hline 8 & 2 & 1.22 & -. & - & -- & 6.8 & -. & -. & -- \\
\hline 23 & 3 & -0.56 & - & - & 6.22 & -- & - & -- & - \\
\hline 25 & 2 & -1.48 & - & - & $\ldots$ & 5.92 & $\cdots$ & $\ldots$ & -. \\
\hline 33 & 2 & -1.39 & -. & - & -- & -- & 5.95 & -- & -- \\
\hline 38 & 4 & -0.01 & -. & - & 6.4 & - & -. & -. & - \\
\hline 45 & 4 & -0.16 & -. & - & - & -- & -. & 6.35 & -. \\
\hline 59 & 0 & 3.30 & - & -- & -- & - & - & -- & 7.48 \\
\hline 64 & 4 & 0.33 & - & $=$ & $\overline{--}$ & 6.51 & -. & - & $\ldots$ \\
\hline 85 & 2 & $\mathrm{t} .12$ & - & 6.77 & -- & -- & -- & - & -- \\
\hline 86 & 4 & -0.41 & - & -- & -. & 6.27 & - & - & -- \\
\hline 89 & 2 & 1.46 & -. & 6.88 & .. & -- & .. & -- & -- \\
\hline 105 & 3 & 0.51 & - & -- & -- & 6.57 & - & -- & - \\
\hline 110 & 4 & 0.00 & - & - & -- & 6.403 & - & - & -. \\
\hline 113 & 4 & -0.38 & .. & -- & -- & 6.28 & - & -. & - \\
\hline 134 & 4 & 0.30 & -. & - & -. & 6.5 & -. & -. & - \\
\hline 138 & 4 & -0.32 & - & - & -- & 6.3 & - & - & - \\
\hline 180 & 4 & -0.10 & - & -. & -- & 6.37 & .. & .. & $=$ \\
\hline 190 & 3 & -0.59 & - & - & 6.21 & -- & - & - & - \\
\hline 193 & 2 & -1.33 & -. & - & 5.97 & - & -- & -. & - \\
\hline 245 & 4 & 0.13 & -. & - & -- & -. & -- & 6.447 & - \\
\hline 247 & 0 & -2.19 & -. & - & .- & 5.69 & .. & -- & $\ldots$ \\
\hline 265 & 4 & 0.30 & -- & - & - & 6.5 & - & - & - \\
\hline 279 & 0 & 2.96 & 7.37 & - & -- & - & - & -. & - \\
\hline 315 & 4 & -0.32 & -. & -- & .. & 6.3 & -- & -- & -. \\
\hline 321 & 4 & -0.44 & -. & 6.26 & -- & -- & .. & -- & - \\
\hline 328 & 3 & 0.91 & -. & -- & - & 6.7 & -- & -. & -- \\
\hline 333 & 4 & 0.36 & -- & - & - & 6.52 & - & $\ldots$ & - \\
\hline 336 & 0 & 29.79 & 16.12 & - & - & - & $=$ & - & - \\
\hline 370 & 2 & 1.49 & -- & - & .. & 6.89 & .. & -. & - \\
\hline 372 & 3 & -0.62 & -- & - & .. & 6.2 & -- & -. & -. \\
\hline
\end{tabular}


Table 15. Statistical summary of reported data for standard reference sample P-38 (low ionic strength constituents) -- continued

P-38 CHLORIDE (Cl) in mg/L

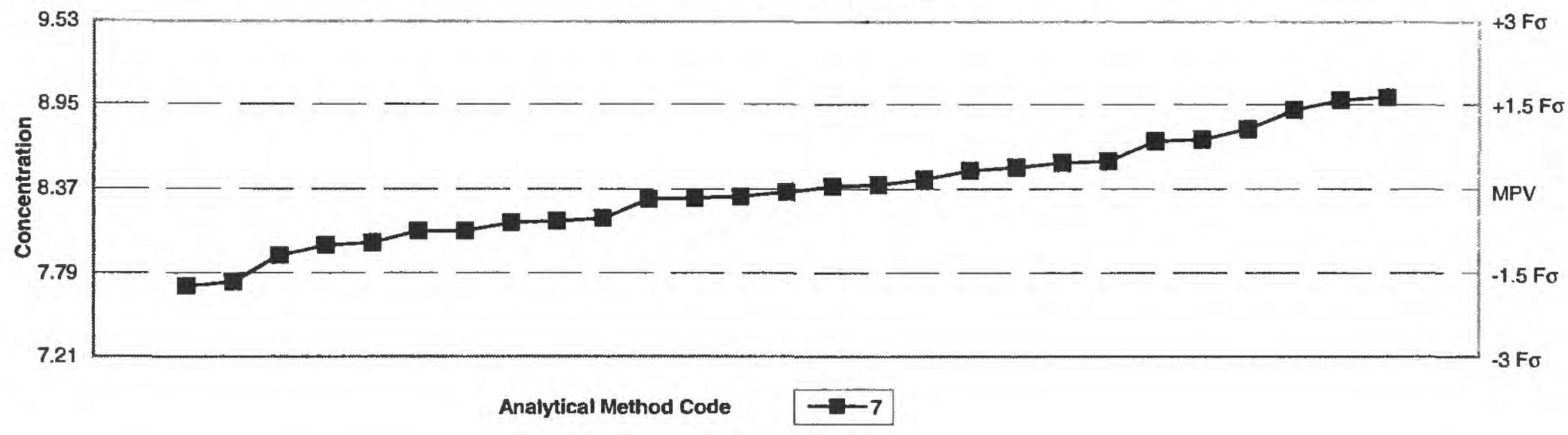

\begin{tabular}{|c|c|c|c|c|}
\hline \multirow[t]{2}{*}{ SUMMARY } & \multicolumn{2}{|c|}{ Methods } & \multicolumn{2}{|c|}{ Statistics } \\
\hline & 0 & Method Codes & & \\
\hline$n=$ & 27 & 00 Other & MPV $=$ & $8.37 \mathrm{mg} / \mathrm{L}$ \\
\hline Minimum $=$ & 29.82 & 07 Ion chromatography & F-pseudosigma = & 0.39 \\
\hline Maximum $=$ & 9 & & Rating criterion = & 0.42 \\
\hline Median $=$ & 8.35 & & $n=$ & 28 \\
\hline \multirow{2}{*}{ F-pseudosigma $=$} & 0.33 & & $\mathrm{Uh}=$ & 8.63 \\
\hline & & & $\mathrm{Lh}=$ & 8.11 \\
\hline
\end{tabular}

\begin{tabular}{|c|c|c|c|c|}
\hline \multirow[b]{2}{*}{ Lab } & \multirow[b]{2}{*}{ Rating } & \multirow[b]{2}{*}{$Z$-value } & \multirow{2}{*}{\multicolumn{2}{|c|}{ Method Codes }} \\
\hline & & & 0 & \\
\hline 1 & 2 & -1.10 & 7.91 & \\
\hline 5 & 4 & 0.05 & 8.39 & \\
\hline 8 & 4 & -0.05 & 8.35 & \\
\hline 23 & 3 & 0.79 & 8.7 & \\
\hline 25 & 4 & 0.07 & 8.4 & \\
\hline 33 & 4 & 0.43 & 8.55 & \\
\hline 45 & 3 & -0.53 & 8.15 & \\
\hline 59 & 1 & -1.53 & 7.73 & \\
\hline 64 & 3 & 0.98 & .- 8.78 & \\
\hline 85 & 4 & 0.31 & .- $\quad 8.5$ & \\
\hline 86 & 2 & 1.46 & -8.98 & \\
\hline 89 & 4 & 0.45 & 8.56 & \\
\hline 105 & 3 & -0.88 & $--\quad 8$ & \\
\hline 110 & 4 & -0.48 & - 8.168 & \\
\hline 113 & 4 & -0.12 & .- 8.32 & \\
\hline 134 & 3 & -0.69 & 8.08 & \\
\hline 138 & 2 & 1.29 & .- 8.91 & \\
\hline 180 & 3 & -0.69 & - $\quad 8.08$ & \\
\hline 190 & 4 & 0.36 & .- 8.52 & \\
\hline 208 & 4 & -0.14 & 8.31 & \\
\hline 247 & 3 & -0.93 & 7.98 & \\
\hline 265 & 1 & -1.60 & 7.7 & \\
\hline 315 & 4 & -0.17 & 8.3 & \\
\hline 321 & 4 & 0.17 & -- 8.44 & \\
\hline 328 & 2 & 1.51 & - & \\
\hline 336 & 0 & 51.25 & 29.82 & \\
\hline 370 & 3 & 0.81 & - 8.71 & \\
\hline 372 & 3 & -0.55 & - 8.14 & \\
\hline
\end{tabular}


Table 15. Statistical summary of reported data for standard reference sample P-38 (low ionic strength constituents) -- continued

P-38 Fluoride $(F)$ in $\mathrm{mg} / \mathrm{L}$

\begin{tabular}{|c|c|c|c|c|c|c|}
\hline \multirow{2}{*}{ SUMMAAY } & \multicolumn{5}{|c|}{ Methods } & \multirow{2}{*}{ Statistics } \\
\hline & $\begin{array}{l}0 \\
0\end{array}$ & 7 & 22 & 40 & Method Codes & \\
\hline$n=$ & 1 & 9 & 1 & 4 & 00 Other & inadequate data \\
\hline Minimum = & 0.93 & 0.012 & 0.026 & 0.01 & 07 Ion chromatography & $(\mathrm{F} \sigma>\mathrm{MPV})$ \\
\hline Maximum $=$ & & 0.22 & & 0.28 & 22 Colorimetric & \\
\hline Median $=$ & & 0.040 & & & 40 Ion selective electrode & \\
\hline F-pseudosigma $=$ & & 0.030 & & & & \\
\hline
\end{tabular}

\begin{tabular}{|c|c|c|c|c|c|c|c|}
\hline \multirow[b]{2}{*}{ Lab } & \multirow[b]{2}{*}{ Rating } & \multirow[b]{2}{*}{ Z-value } & \multicolumn{5}{|c|}{ Method Codes } \\
\hline & & & 0 & 7 & 22 & 40 & \\
\hline 1 & $\mathrm{NR}$ & -0.26 & - & - & 0.026 & - & \\
\hline 2 & NR & 0.00 & -. & 0.037 & - & -- & \\
\hline 8 & NR & -- & -- & $<0.3$ & -- & -. & \\
\hline 23 & NR & -. & -- & $<0.15$ & -. & -. & \\
\hline 25 & $\mathrm{NR}$ & 0.07 & -. & 0.04 & -- & -- & \\
\hline 33 & $\mathrm{NR}$ & -0.36 & -. & 0.022 & - & - & \\
\hline 45 & $\mathrm{NR}$ & 0.31 & -- & 0.05 & -- & -. & \\
\hline 59 & NR & 5.75 & -- & - & - & 0.28 & \\
\hline 85 & NR & -0.17 & -- & -- & - & 0.03 & \\
\hline 86 & NR & 4.33 & -- & 0.22 & - & -- & \\
\hline 89 & NR & - & $=$ & -- & $<0.1$ & - & \\
\hline 105 & NR & -. & -- & $<0.20$ & - & - & \\
\hline 134 & NR & -- & -- & - & - & $<0.1$ & \\
\hline 138 & NR & -- & -- & -- & & $<0.050$ & \\
\hline 180 & NR & -0.59 & -- & 0.012 & -- & -. & \\
\hline 190 & NR & -0.64 & - & - & $=$ & 0.01 & \\
\hline 247 & NR & -0.40 & -- & 0.02 & - & -. & \\
\hline 328 & NR & -0.26 & -- & - & - & 0.026 & \\
\hline 336 & NR & 21.13 & 0.93 & -. & - & -- & \\
\hline 370 & NR & 0.59 & -- & 0.062 & - & -- & \\
\hline 372 & NR & 1.49 & - & 0.1 & - & -- & \\
\hline
\end{tabular}


Table 15. Statistical summary of reported data for standard reference sample P-38 (low ionic strength constituents) -- continued

P-38 POTASSIUM (K) in $\mathrm{mg} / \mathrm{L}$

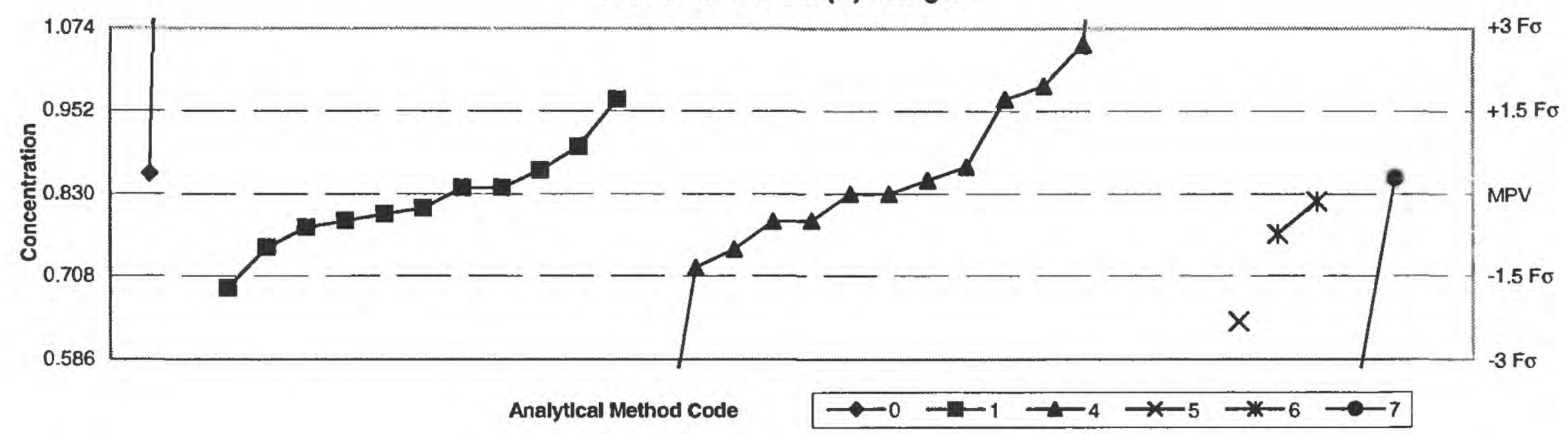

\begin{tabular}{|c|c|c|c|c|c|c|c|c|c|}
\hline \multirow[t]{2}{*}{ SUMMARY } & \multicolumn{7}{|c|}{ Methods } & \multicolumn{2}{|c|}{ Statlstlcs } \\
\hline & 0 & 1 & 4 & 5 & 6 & 7 & Method Codes & & \\
\hline $\mathrm{n}=$ & 2 & $\overline{11}$ & 14 & $\overline{1}$ & 2 & 2 & 00 Other & MPV $=$ & $0.830 \mathrm{mg} / \mathrm{L}$ \\
\hline Minimum $=$ & 0.86 & 0.69 & 0.39 & 0.64 & 0.77 & 0.54 & 01 Atomic absorption; direct, air & dosigma $=$ & 0.081 \\
\hline Maximum $=$ & 3.419 & 0.97 & 3.38 & & 0.819 & 0.854 & 04 Inductively coupled plasma & $n=$ & 32 \\
\hline Median = & & 0.809 & 0.840 & & & & 05 Direct current piasma & $U h=$ & 0.885 \\
\hline F-pseudosigma $=$ & & 0.050 & 0.149 & & & & $\begin{array}{l}06 \text { Inductively coupled plasma/mass spectrometry } \\
07 \text { lon chromatography }\end{array}$ & $\operatorname{Lh}=$ & 0.775 \\
\hline
\end{tabular}

\begin{tabular}{|c|c|c|c|c|c|c|c|c|}
\hline \multirow[b]{2}{*}{ Lab } & \multirow[b]{2}{*}{ Rating } & \multirow[b]{2}{*}{$Z$-value } & \multicolumn{6}{|c|}{ Method Codes } \\
\hline & & & 0 & 1 & 4 & 5 & 6 & 7 \\
\hline 1 & 4 & -0.49 & - & 0.79 & -- & - & - & -- \\
\hline 2 & 4 & 0.29 & -- & -- & -- & -. & - & 0.854 \\
\hline 5 & 0 & 31.30 & -- & -- & 3.38 & -- & -- & -- \\
\hline 8 & 4 & 0.25 & - & -. & 0.85 & - & - & -- \\
\hline 23 & 1 & 1.72 & - & 0.97 & - & - & - & -- \\
\hline 25 & 1 & 1.96 & -. & $\cdots$ & 0.99 & $\cdots$ & - & - \\
\hline 33 & 0 & -2.33 & -- & -- & - & 0.64 & -. & -. \\
\hline 38 & 4 & -0.37 & - & 0.8 & -- & -- & -. & -- \\
\hline 45 & 4 & -0.14 & - & -- & - & - & 0.819 & -- \\
\hline 59 & 0 & -3.56 & -. & - & -. & -. & .. & 0.54 \\
\hline 64 & 1 & -1.72 & - & 0.69 & $=$ & - & -- & -- \\
\hline 85 & 3 & 0.86 & -- & 0.9 & -- & -. & -- & -- \\
\hline 86 & 4 & 0.00 & - & - & 0.83 & -. & -- & -- \\
\hline 89 & 4 & 0.43 & -- & 0.865 & -. & - & -- & -. \\
\hline 105 & NR & -- & - & - & $<1.00$ & - & -. & -- \\
\hline 110 & 4 & 0.11 & $\because$ & 0.839 & - & - & - & - \\
\hline 113 & 4 & -0.50 & - & -- & 0.789 & - & -- & - \\
\hline 134 & 4 & -0.26 & -- & 0.809 & -- & - & - & -- \\
\hline 138 & 4 & -0.49 & -. & - & 0.79 & - & -- & - \\
\hline 180 & 2 & -1.02 & -. & $=$ & 0.747 & - & 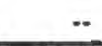 & - \\
\hline 190 & 3 & -0.61 & $\ldots$ & 0.78 & - & - & $\because$ & - \\
\hline 193 & 4 & 0.11 & -. & 0.839 & -- & $\cdots$ & -- & - \\
\hline 245 & 3 & -0.73 & -. & - & -- & $\ldots$ & 0.77 & - \\
\hline 247 & 2 & -1.35 & -. & - & 0.72 & $\ldots$ & - & - \\
\hline 265 & 4 & 0.00 & -. & - & 0.83 & - & - & - \\
\hline 279 & 4 & 0.37 & 0.86 & - & -- & $\ldots$ & - & - \\
\hline 315 & 1 & 1.72 & - & -. & 0.97 & $\ldots$ & -- & - \\
\hline 321 & 3 & -0.98 & - & 0.75 & -- & $\ldots$ & -- & -. \\
\hline 328 & 0 & 9.82 & -- & - & 1.63 & $\ldots$ & - & -. \\
\hline 333 & 4 & 0.49 & -- & .. & 0.87 & -. & - & - \\
\hline 336 & 0 & 31.78 & 3.419 & 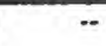 & - & - & $\omega$ & - \\
\hline 370 & 0 & 2.70 & -- & -. & 1.05 & - & -- & - \\
\hline 372 & 0 & -5.40 & - & .. & 0.39 & - & -- & - \\
\hline
\end{tabular}


Table 15. Statistical summary of reported data for standard reference sample P-38 (low ionic strength constituents) - continued

P-38 MAGNESIUM $(\mathrm{Mg})$ in $\mathrm{mg} / \mathrm{L}$

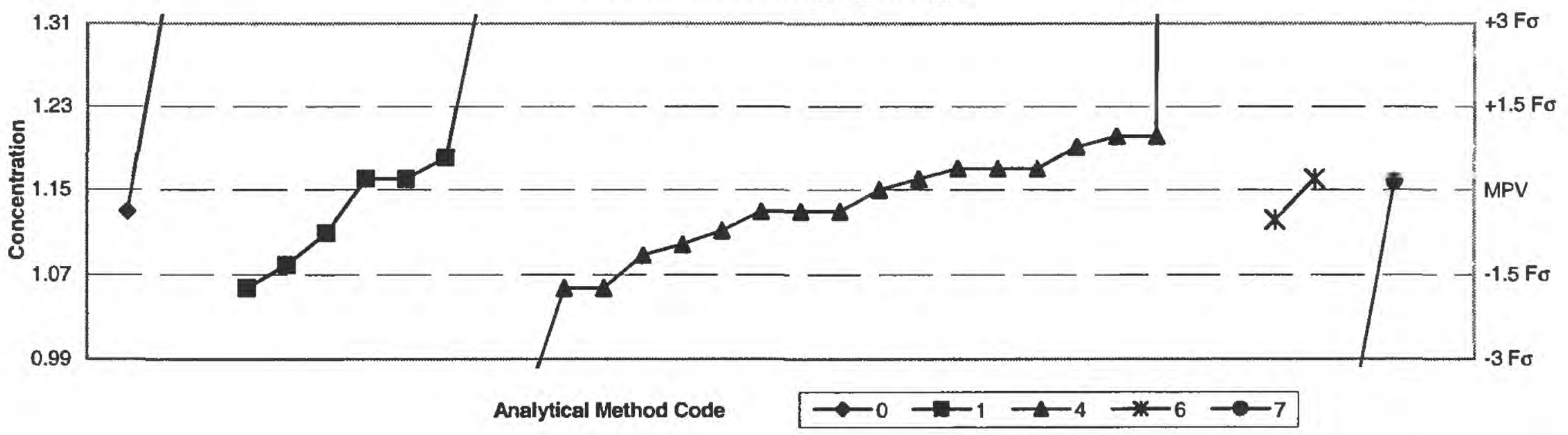

\begin{tabular}{|c|c|c|c|c|c|c|c|c|c|}
\hline \multirow[t]{2}{*}{ SUMMARY } & \multicolumn{7}{|c|}{ Methods } & \multicolumn{2}{|c|}{ Statistles } \\
\hline & 0 & 1 & 4 & 5 & 6 & 7 & Method Codes & & \\
\hline$n=$ & 3 & 7 & 18 & 1 & 2 & 2 & 00 Other & $P V=$ & $1.15 \mathrm{mg} / \mathrm{L}$ \\
\hline Minimum = & 1.13 & 1.06 & 0.95 & 0.795 & 1.122 & 0.96 & 01 Atomic absorption: direct, air & $m a=$ & 0.05 \\
\hline Maximum = & 4.34 & 1.36 & 5.67 & & 1.16 & 1.158 & 04 Inductively coupled plasma & ion $=$ & 0.06 \\
\hline Median $=$ & & 1.16 & 1.14 & & & & 05 Direct current plasma & $n=$ & 33 \\
\hline \multirow[t]{2}{*}{ F-pseudosigma $=$} & & 0.06 & 0.05 & & & & 06 Inductively coupled plasma/mass spectrometry & Un $=$ & 1.17 \\
\hline & & & & & & & 07 Ion chromatography & $\operatorname{Lh}=$ & 1.10 \\
\hline
\end{tabular}

\begin{tabular}{|c|c|c|c|c|c|c|c|c|}
\hline \multirow[b]{2}{*}{ Lab } & \multirow[b]{2}{*}{ Rating } & \multirow[b]{2}{*}{ Z-value } & \multicolumn{6}{|c|}{ Method Codes } \\
\hline & & & 0 & 1 & 4 & 5 & 6 & 7 \\
\hline 1 & 4 & 0.35 & - & - & 1.17 & - & - & - \\
\hline 2 & 4 & 0.14 & - & - & -- & -- & - & 1.158 \\
\hline 5 & 0 & 78.61 & - & - & 5.67 & -- & - & - \\
\hline 8 & 3 & 0.87 & - & .. & 1.2 & -- & - & - \\
\hline 23 & 3 & -0.70 & - & 1.11 & - & -- & - & - \\
\hline 25 & 2 & -1.04 & - & - & 1.09 & -. & - & $\overline{-}$ \\
\hline 33 & 0 & -6.17 & - & -- & -- & 0.795 & -- & - \\
\hline 38 & 2 & -1.20 & - & 1.081 & -. & -- & - & - \\
\hline 45 & 4 & 0.17 & - & -- & - & -- & 1.16 & - \\
\hline 59 & 0 & -3.30 & - & - & - & - & - & 0.96 \\
\hline 64 & 4 & -0.35 & - & - & 1.13 & - & - & - \\
\hline 85 & 3 & 0.52 & - & 1.18 & -- & - & -- & - \\
\hline 86 & 4 & 0.35 & - & -- & 1.17 & -- & -- & - \\
\hline 89 & 0 & 3.65 & - & 1.36 & -- & - & -- & - \\
\hline 105 & 3 & 0.70 & - & - & 1.19 & - & -- & - \\
\hline 110 & 3 & -0.65 & - & - & 1.112 & - & - & - \\
\hline 113 & 3 & -0.87 & - & - & 1.1 & - & - & -- \\
\hline 134 & 4 & -0.35 & - & - & 1.13 & -- & -- & - \\
\hline 138 & 4 & 0.17 & - & - & 1.16 & -- & - & -- \\
\hline 180 & 4 & -0.35 & - & -- & 1.13 & - & $\cdots$ & - \\
\hline 190 & 4 & 0.17 & - & 1.16 & - & - & - & - \\
\hline 193 & 4 & 0.17 & -- & 1.16 & - & -- & - $\quad--$ & - \\
\hline 245 & 4 & -0.49 & -- & - & - & -- & 1.122 & - \\
\hline 247 & 0 & -3.48 & -- & -- & 0.95 & -. & - $\quad-$ & -- \\
\hline 265 & 4 & 0.00 & - & $\ldots$ & 1.15 & -- & - & - \\
\hline 279 & $\overline{0}$ & 3.30 & 1.34 & - & - & - & - & - \\
\hline 315 & 3 & 0.87 & -- & -- & 1.2 & - & - & - \\
\hline 321 & 1 & -1.57 & - & 1.06 & -- & -. & -- & - \\
\hline 328 & 4 & -0.35 & 1.13 & -. & -- & -- & -- & - \\
\hline 333 & 4 & 0.35 & -- & - & 1.17 & -. & - & $=$ \\
\hline 336 & 0 & 55.48 & 4.34 & - & - & -- & - & - \\
\hline 370 & 1 & -1.57 & -. & - & 1.06 & -- & - & -- \\
\hline 372 & 1 & -1.57 & - & - & 1.06 & -- & - & - \\
\hline
\end{tabular}


Table 15. Statistical summary of reported data for standard reference sample P-38 (low ionic strength constituents) -- continued

P-38 SODIUM $(\mathrm{Na})$ in $\mathrm{mg} / \mathrm{L}$

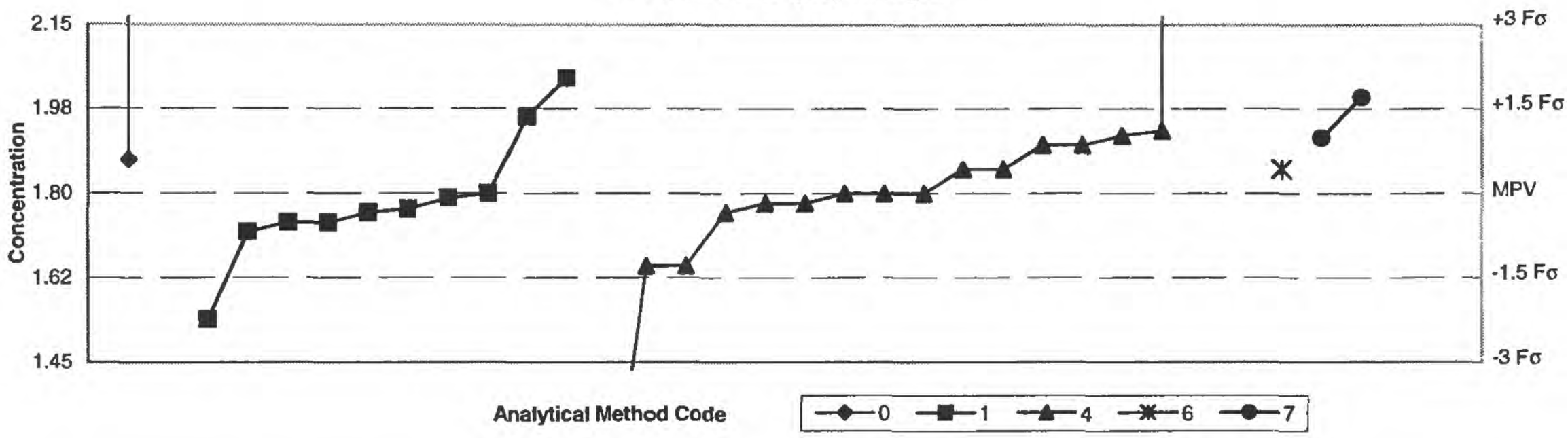

\begin{tabular}{|c|c|c|c|c|c|c|c|c|c|}
\hline \multirow[t]{2}{*}{ SUMMARY } & \multicolumn{7}{|c|}{ Methods } & \multicolumn{2}{|c|}{ Statistics } \\
\hline & 0 & 1 & 4 & 5 & 6 & 7 & Method Codes & & \\
\hline $\mathbf{n}=$ & 2 & 10 & 16 & 1 & 1 & 2 & Do Other & MPV $=$ & $1.80 \mathrm{mg} / \mathrm{L}$ \\
\hline Minimum = & 1.87 & 1.54 & 1.07 & 0.676 & 1.85 & 1.915 & 01 Atomic absorption: direct, air & dosigma $=$ & 0.12 \\
\hline Maximum $=$ & 25.5 & 2.04 & 24 & & & 2 & 04 Inductively coupled plasma & $n=$ & 32 \\
\hline Median $=$ & & 1.76 & 1.80 & & & & 05 Direct current plasma & Un $=$ & 1.91 \\
\hline F-pseudosigma $=$ & & 0.04 & 0.10 & & & & $\begin{array}{l}06 \text { Inductively coupled plasma/mass spectrometry } \\
07 \text { Ion chromatography }\end{array}$ & $\operatorname{Lh}=$ & 1.75 \\
\hline
\end{tabular}

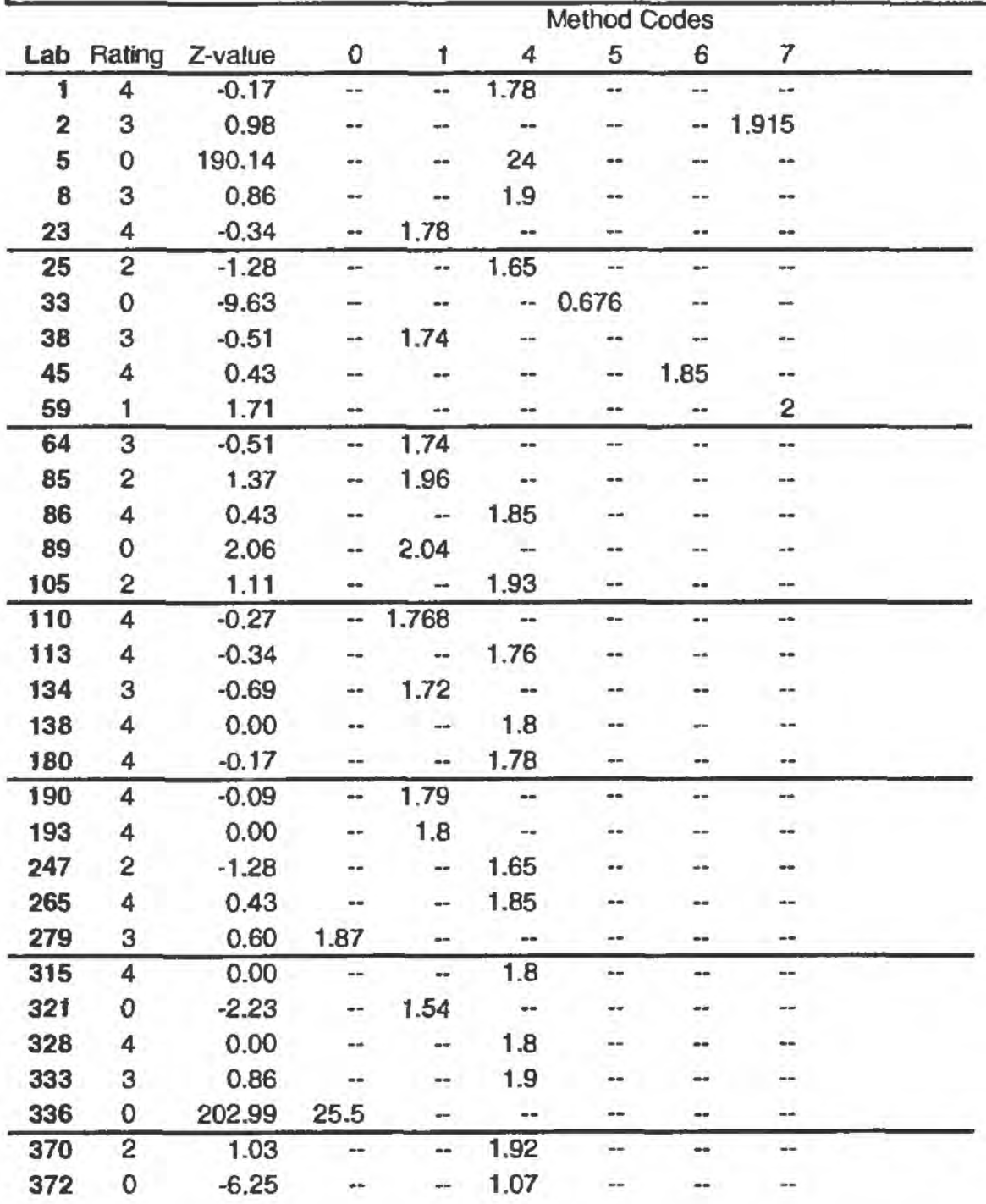


Table 15. Statistical summary of reported data for standard reference sample P-38 (low ionic strength constituents) - continued

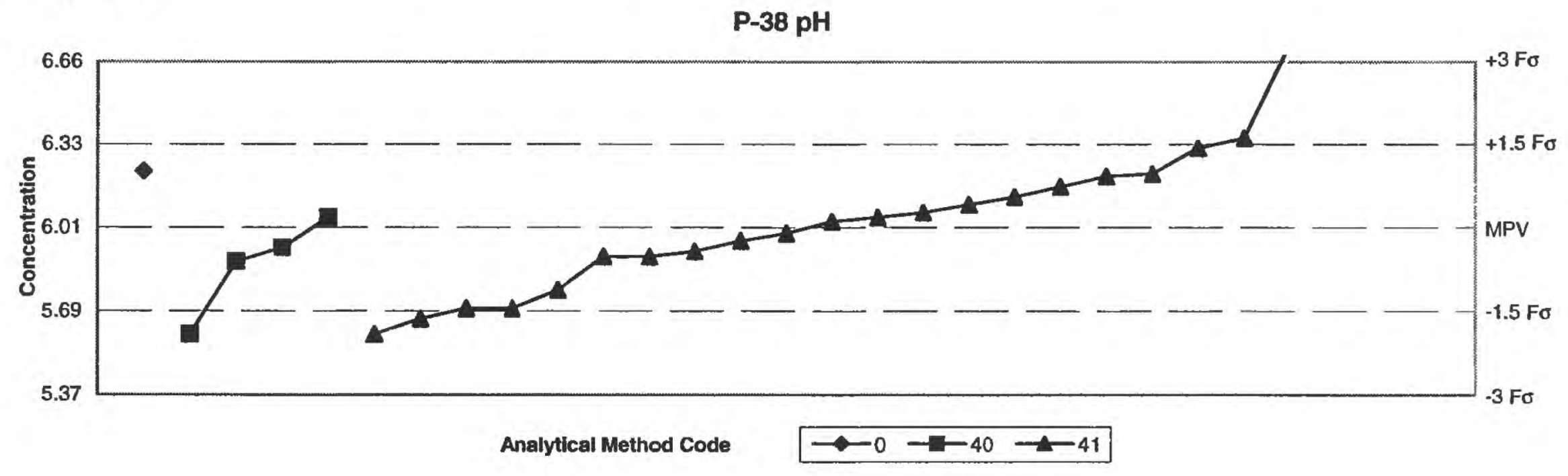

\begin{tabular}{|c|c|c|c|c|c|c|}
\hline \multirow[t]{2}{*}{ SUMMARY } & \multicolumn{4}{|c|}{ Methods } & \multicolumn{2}{|c|}{ Statlstics } \\
\hline & \multicolumn{4}{|r|}{ Method Codes } & \\
\hline$n=$ & 1 & 4 & 21 & 00 Other & MPV $=$ & 6.01 \\
\hline Minimum $=$ & 6.23 & 5.6 & 5.6 & 40 Ion selective electrode & F-pseudosigma $=$ & 0.21 \\
\hline Maximum $=$ & & 6.05 & 6.73 & 4t Electrometric & Rating criterion = & 0.30 \\
\hline Median = & & & 6.03 & & $n=$ & 26 \\
\hline F-pseudosigma $=$ & & & 0.20 & & $\mathrm{Uh}=$ & 6.17 \\
\hline & & & & & $\mathrm{Lh}=$ & 5.88 \\
\hline
\end{tabular}

\begin{tabular}{|c|c|c|c|c|c|c|}
\hline \multirow[b]{2}{*}{ Lab } & \multirow[b]{2}{*}{ Rating } & \multirow[b]{2}{*}{$Z$-value } & \multicolumn{4}{|c|}{ Method Codes } \\
\hline & & & 0 & 40 & 41 & \\
\hline 2 & 4 & 0.07 & -. & -- & 6.034 & \\
\hline 5 & 2 & -1.17 & - & -- & 5.66 & \\
\hline 8 & 0 & 2.39 & .. & -. & 6.73 & \\
\hline 23 & 2 & 1.02 & -. & -- & 6.32 & \\
\hline 25 & 3 & 0.53 & - & 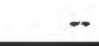 & 6.17 & \\
\hline 33 & 3 & 0.66 & - & - & 6.21 & \\
\hline 38 & 4 & 0.29 & -. & .- & 6.1 & \\
\hline 45 & 4 & -0.17 & -. & -. & 5.96 & \\
\hline 59 & 4 & -0.07 & -. & -. & 5.99 & \\
\hline 64 & 4 & 0.19 & - & - & 6.07 & \\
\hline 85 & 4 & 0.39 & .. & - & 6.13 & \\
\hline 86 & 4 & -0.37 & -. & - & 5.9 & \\
\hline 89 & 2 & -1.04 & -- & -- & 5.7 & \\
\hline 105 & 4 & -0.37 & .- & - & 5.9 & \\
\hline 110 & 4 & -0.26 & .. & 5.935 & .- & \\
\hline 134 & 4 & -0.44 & - & 5.88 & -. & \\
\hline 138 & 4 & 0.13 & .. & -- & 6.05 & \\
\hline 180 & 4 & 0.13 & .- & 6.05 & .. & \\
\hline 190 & 2 & -1.37 & - & -- & 5.6 & \\
\hline 247 & 3 & 0.69 & .. & - & 6.22 & \\
\hline 321 & 2 & -1.04 & 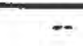 & + & 5.7 & \\
\hline 328 & 2 & -1.37 & .- & 5.6 & .. & \\
\hline 333 & 4 & -0.31 & .. & -. & 5.92 & \\
\hline 336 & 3 & 0.73 & 6.23 & .. & .- & \\
\hline 370 & 3 & -0.81 & .. & .. & 5.77 & \\
\hline 372 & 2 & t.16 & - & $\cdots$ & 6.36 & \\
\hline
\end{tabular}


Table 15. Statistical summary of reported data for standard reference sample P-38 (low ion/c strength constituents) - continued

P-38 ORTHOPHOSPHATE as ORTHOPHOSPHORUS (PO4 as P) in mg/L

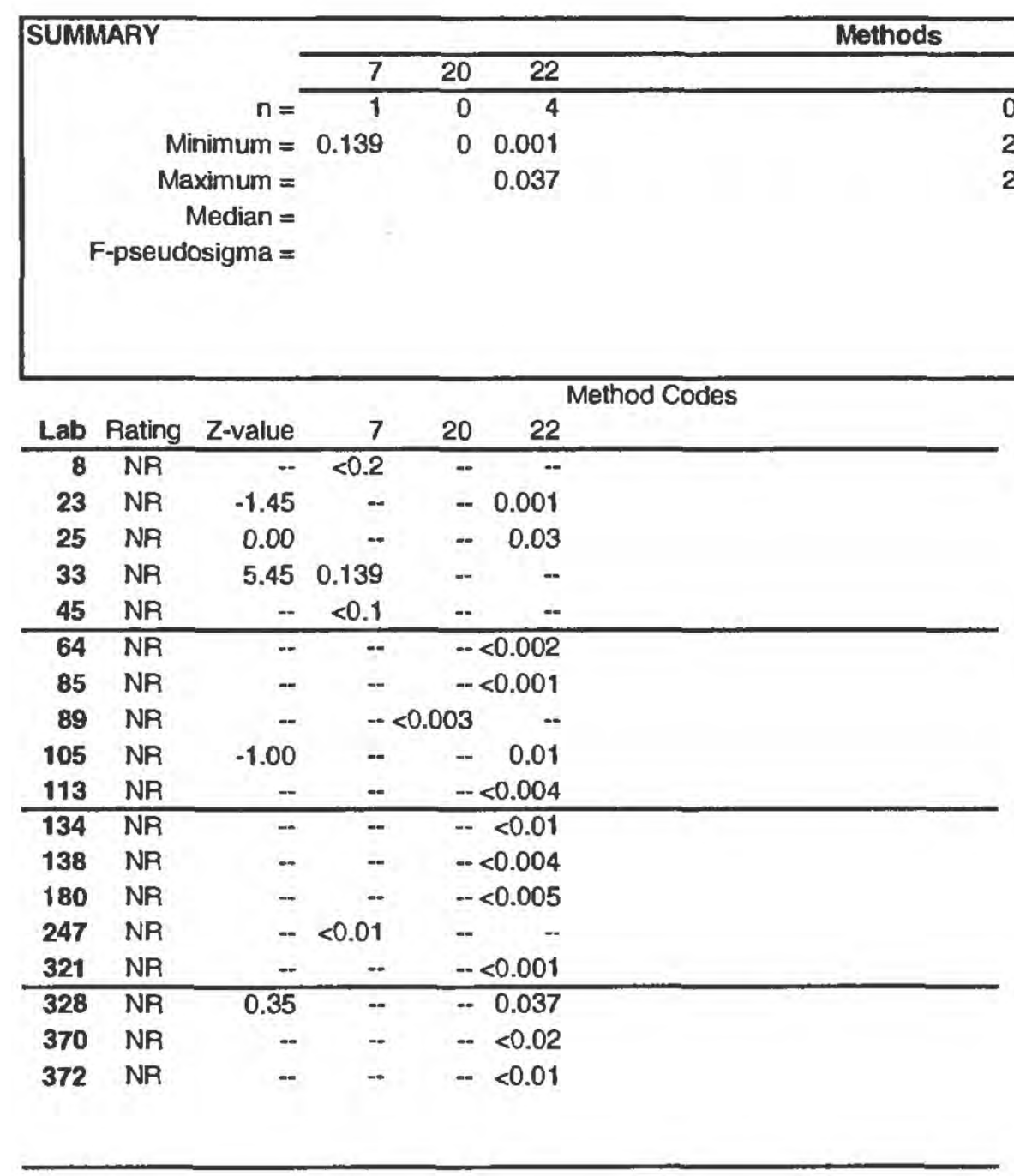


Table 15. Statistical summary of reported data for standard reference sample P-38 (low ionic strength constituents) - continued

\section{P-38 SULFATE $\left(\mathrm{SO}_{4}\right)$ In mg/L}

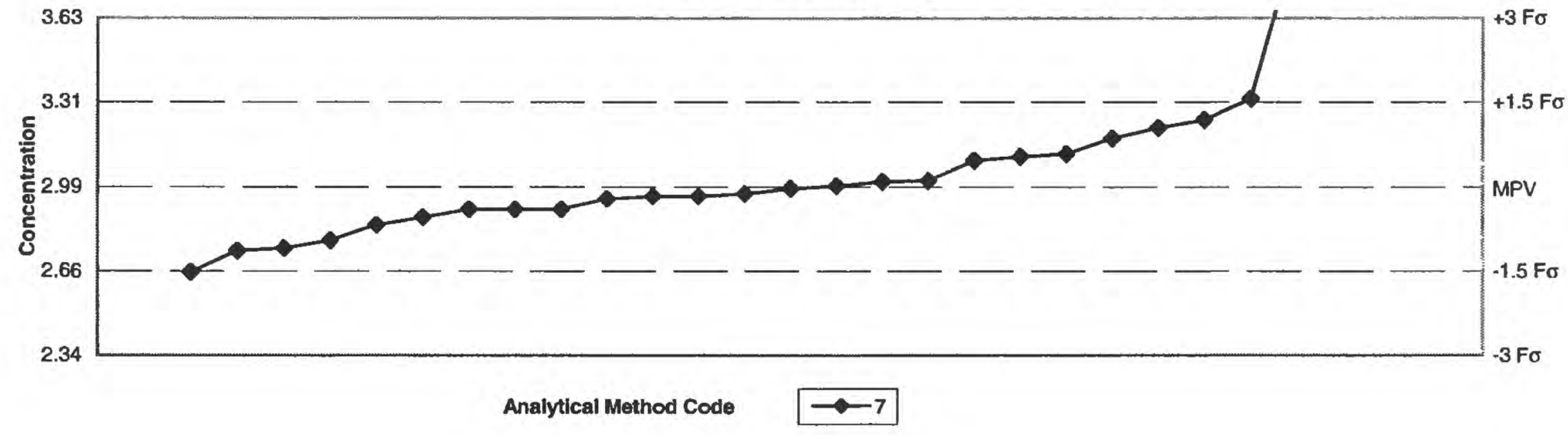

\begin{tabular}{|c|c|c|c|c|}
\hline \multirow[t]{2}{*}{ SUMMARY } & \multicolumn{2}{|c|}{ Methods } & \multicolumn{2}{|c|}{ Statistlcs } \\
\hline & 0 & Method Codes & & \\
\hline$n=$ & 127 & 00 Other & MPV $=$ & $2.99 \mathrm{mg} / \mathrm{L}$ \\
\hline Minimum $=1$ & $10.86 \quad 2.66$ & 07 Ion chromatography & F-pseudosigma $=$ & 0.21 \\
\hline Maximum = & 4.44 & & $n=$ & 28 \\
\hline Median $=$ & 2.98 & & $U h=$ & 3.19 \\
\hline F-pseudosigma $=$ & 0.18 & & $L h=$ & 2.90 \\
\hline
\end{tabular}

\begin{tabular}{rrrrrr}
\hline Lab & Rating & Z-value & \multicolumn{1}{c}{0} & 7 & Method Codes \\
\hline 1 & 2 & -1.09 & - & 2.75 \\
2 & 4 & 0.47 & - & 3.086 \\
5 & 3 & -0.53 & - & 2.87 \\
8 & 2 & 1.05 & - & 3.21 \\
23 & NR & - & - & $<5.0$ \\
\hline 25 & NR & - & - & $<5.0$ \\
33 & 2 & 1.19 & - & 3.24 \\
45 & 4 & -0.02 & - & 2.98 \\
59 & 3 & -0.95 & - & 2.78 \\
64 & 3 & 0.58 & - & 3.11 \\
\hline 85 & 4 & -0.40 & -- & 2.9 \\
86 & 0 & 5.98 & - & 4.27 \\
89 & 4 & -0.16 & -- & 2.95 \\
105 & 4 & 0.12 & -- & 3.01 \\
110 & 4 & 0.10 & - & 3.006 \\
\hline 113 & 4 & -0.40 & -- & 2.9 \\
134 & 4 & 0.02 & - & 2.99 \\
138 & 4 & -0.21 & -- & 2.94 \\
180 & 2 & -1.14 & -- & 2.74 \\
190 & 4 & -0.16 & - & 2.95 \\
\hline 193 & 4 & -0.12 & - & 2.96 \\
208 & NR & -- & - & $<3$ \\
247 & 3 & -0.67 & -- & 2.84 \\
265 & 4 & -0.40 & - & 2.9 \\
315 & 3 & 0.53 & - & 3.1 \\
\hline 321 & 1 & -1.51 & -- & 2.66 \\
328 & 0 & 6.77 & -- & 4.44 \\
333 & 3 & 0.86 & - & 3.17 \\
336 & 0 & 36.63 & 10.86 & -- \\
370 & 1 & 1.56 & -- & 3.32 \\
\hline 372 & 0 & 4.49 & -- & 3.95 \\
& & & & \\
\hline 10
\end{tabular}


Table 15. Statistical summary of reported data for standard reference sample P-38 (low ionic strength constituents) - continued

\section{P-38 SPECIFIC CONDUCTANCE in $\mu$ S/cm}

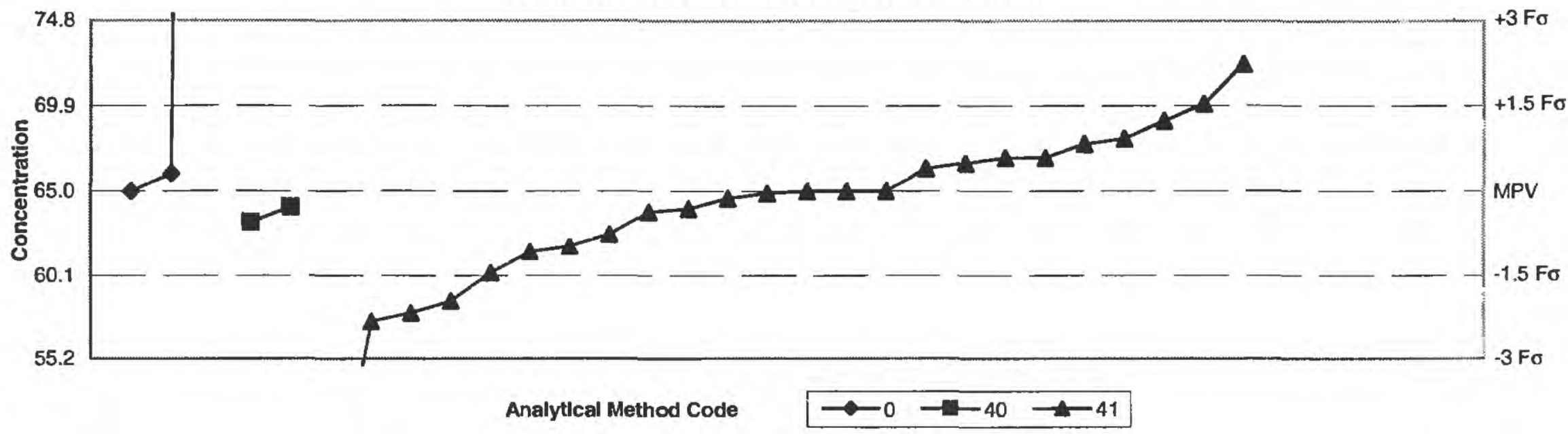

\begin{tabular}{|c|c|c|c|c|c|c|}
\hline \multirow[t]{2}{*}{ SUMMARY } & \multicolumn{4}{|c|}{ Methods } & \multicolumn{2}{|c|}{ Statistics } \\
\hline & 0 & 40 & 41 & Method Codes & & \\
\hline$n=$ & 3 & 2 & 24 & 00 Other & MPV $=$ & $65.0 \mu \mathrm{S} / \mathrm{cm}$ \\
\hline Minimum = & 65 & 63.2 & 45 & 40 Ion selective electrode & F-pseudosigma $=$ & 3.3 \\
\hline Maximum $=$ & 244.6 & 64.1 & 72.3 & 41 Electrometric & $n=$ & 29 \\
\hline Median $=$ & & & 65.0 & & Uh $=$ & 66.9 \\
\hline F-pseudosigma $=$ & & & 3.9 & & $\operatorname{Lh}=$ & 62.5 \\
\hline
\end{tabular}

\begin{tabular}{|c|c|c|c|c|c|}
\hline \multirow[b]{2}{*}{ Lab } & \multirow[b]{2}{*}{ Rating } & \multirow[b]{2}{*}{ Z-value } & \multicolumn{3}{|r|}{ Method Codes } \\
\hline & & & 0 & 40 & 41 \\
\hline 1 & 3 & 0.58 & $\overline{--}$ & -- & 66.9 \\
\hline 2 & 2 & -1.45 & -. & - & 60.27 \\
\hline 5 & 1 & -1.96 & -- & - & 58.6 \\
\hline 8 & 0 & -2.33 & -- & - & 57.4 \\
\hline 23 & 3 & 0.83 & -- & - & 67.7 \\
\hline 25 & 4 & -0.31 & - & $\overline{--}$ & 64 \\
\hline 33 & 0 & 2.24 & -. & -- & 72.3 \\
\hline 38 & 4 & -0.37 & -. & - & 63.8 \\
\hline 45 & 1 & 1.53 & - & - & 70 \\
\hline 59 & 2 & 1.23 & - & - & 69 \\
\hline 64 & 3 & -0.77 & - & - & 62.5 \\
\hline 85 & 4 & 0.31 & 66 & .. & -. \\
\hline 86 & 3 & 0.58 & -. & -- & 66.9 \\
\hline 89 & 4 & -0.12 & -- & - & 64.6 \\
\hline 105 & 4 & 0.49 & - & - & 66.6 \\
\hline 110 & 2 & -1.07 & - & - & 61.5 \\
\hline 113 & 4 & 0.40 & - & - & 66.3 \\
\hline 134 & 4 & -0.28 & -- & 64.1 & -- \\
\hline 138 & 4 & -0.03 & -- & .- & 64.9 \\
\hline 180 & 0 & -6.13 & - & -- & 45 \\
\hline 190 & 3 & -0.55 & $\ldots$ & 63.2 & - \\
\hline 193 & 4 & 0.00 & -- & -- & 65 \\
\hline 247 & 4 & 0.00 & -- & - & 65 \\
\hline 321 & 0 & -2.18 & - & -- & 57.9 \\
\hline 328 & 4 & 0.00 & 65 & - & -- \\
\hline 333 & 3 & -0.98 & - & - & 61.8 \\
\hline 336 & 0 & 55.06 & 244.6 & -- & -- \\
\hline 370 & 3 & 0.92 & - & - & 68 \\
\hline 372 & 4 & 0.00 & - & .- & 65 \\
\hline
\end{tabular}


Table 16. Statistical summary of reported data for standard reference sample HG-34 (mercury)

Hg-34 MERCURY $(\mathrm{Hg})$ in $\mu \mathrm{g} / \mathrm{L}$

\begin{tabular}{|c|c|c|c|c|}
\hline \multirow[t]{2}{*}{ SUMMAAY } & \multicolumn{3}{|r|}{ Methods } & \multirow[t]{2}{*}{ Statistics } \\
\hline & 6 & 9 & Method Codes & \\
\hline$n=$ & $\overline{2}$ & 7 & 06 inductively coupled plasma/mass spectrometry & inadequate date \\
\hline Minimum $=$ & 0.032 & $0.011 \quad 0.011$ & OB Atomic absorption: cold vapor & $($ Fo $>$ MPV) \\
\hline Maximum $=$ & 0.15 & $1.63 \quad 0.034$ & 09 Atomic fluorescence & \\
\hline Median $=$ & & $0.100 \quad 0.027$ & & \\
\hline F-pseudosigma $=$ & & $0.819 \quad 0.002$ & & \\
\hline
\end{tabular}

\begin{tabular}{|c|c|c|c|c|c|}
\hline \multirow[b]{2}{*}{ Lab } & \multirow[b]{2}{*}{ Rating } & \multirow[b]{2}{*}{$Z$-value } & \multirow{2}{*}{\multicolumn{3}{|c|}{ Method Codes }} \\
\hline & & & 6 & & \\
\hline 1 & NR & -0.03 & 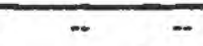 & 0.027 & \\
\hline 5 & NR & 28.78 & 1.63 & - & \\
\hline 8 & NR & 2.19 & 0.15 & - & \\
\hline 23 & NR & 1.29 & 0.1 & -. & \\
\hline 26 & NR & -- & $<0.2$ & - & \\
\hline 32 & NR & -. & $<0.05$ & -. & \\
\hline 45 & NR & 0.07 & 0.032 & -. & \\
\hline 46 & NR & -0.31 & - & 0.011 & \\
\hline 59 & NR & -0.05 & -- & 0.025 & \\
\hline 89 & NR & -- & $<0.2$ & -- & \\
\hline 97 & NR & -. & $<0.1$ & - & \\
\hline 105 & NR & 19.79 & 1.13 & -. & \\
\hline 134 & NR & -- & $<0.1$ & - & \\
\hline 138 & NR & 0.00 & -- & 0.028 & \\
\hline 147 & NR & 0.10 & -- & 0.034 & \\
\hline 180 & NR & - & $-<0.050$ & - & \\
\hline 193 & NR & -0.06 & -0.025 & -- & \\
\hline 245 & NR & -0.06 & $-\quad-$ & 0.025 & \\
\hline 247 & NR & -- & $-<<0.20$ & -- & \\
\hline 304 & NR & 0.00 & $-\quad-$ & 0.028 & \\
\hline 356 & $\overline{N R}$ & -0.31 & -0.011 & - & \\
\hline 370 & NR & - & $-<100$ & - & \\
\hline 372 & NR & -- & -0.0002 & - & \\
\hline
\end{tabular}


Table 17. Most probable values for constituents and propertles in standard reference samples distributed in March 2002

[MPV, most probable value; $n$, number of samples; $\mu \mathrm{g} / \mathrm{L}$, microgram per liter; $\mathrm{mg} / \mathrm{L}$, milligram per liter; $\mu \mathrm{S} / \mathrm{cm}$, microsiemens per centimeter at 25 degres Celsius; ( ) rating criterion]

T-169

\begin{tabular}{|c|c|}
\hline $\begin{array}{r}\text { Analyte }= \\
\text { MPV }= \\
\text { F-pseudosigma }= \\
n=\end{array}$ & $\begin{array}{l}\text { Silver } \\
3.90 \mu g / L \\
0.24 \\
39\end{array}$ \\
\hline $\begin{array}{r}\text { Analyte }= \\
\text { MPV }= \\
\text { F-pseudosigma }= \\
n=\end{array}$ & $\begin{array}{l}\text { Beryllium } \\
9.61 \text { Ag/L } \\
0.56 \\
42\end{array}$ \\
\hline
\end{tabular}

Analyte $=\quad$ Copper

MPV $=\quad 14.3 \mu g / L$

F-pseudosigma $=\quad 0.8$

$n=\quad 48$

\section{Analyte = Manganese} MPV $=\quad 27.0 \mu \mathrm{g} / \mathrm{L}$

$\begin{array}{ll}\text { F-pseudosigma }= & 0.9(1.4)\end{array}$

$$
n=\quad 48
$$

$\begin{aligned} \text { Analyte }= & \text { Antimony } \\ \text { MPV }= & 3.33 \mathrm{~kg} / \mathrm{L} \\ \text { F-pseudosigma }= & 0.44 \\ \mathrm{n}= & 31\end{aligned}$

\begin{tabular}{|c|c|}
\hline $\begin{array}{r}\text { Analyte }= \\
\text { MPV }= \\
\text { F-pseudosigma }= \\
n=\end{array}$ & $\begin{array}{l}\text { Uranlum } \\
1.71 \mu \mathrm{g} / \mathrm{L} \\
0.11 \\
14\end{array}$ \\
\hline
\end{tabular}

\section{M-162}

$\begin{array}{rlrl}\text { Analyte } & = & & \text { Alkallinity } \\ \text { MPV }= & & 11.0 \mathrm{mg} / \mathrm{L} \\ \text { F-pseudosigma } & = & 1.7 \\ \mathrm{n}= & & 53\end{array}$

$\begin{aligned} \text { Analyte }= & \text { Potassium } \\ \text { MPV }= & 3.20 \mathrm{mg} / \mathrm{L} \\ \text { dosigma }= & 0.17 \\ \mathrm{n}= & 55\end{aligned}$

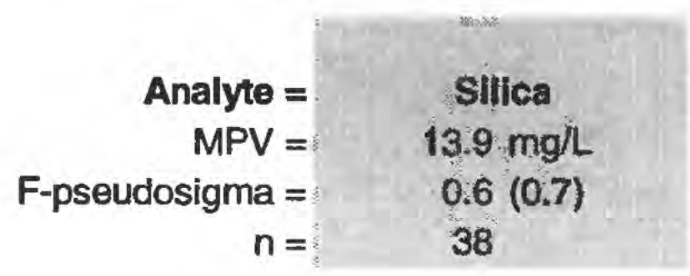

$$
n=\quad 38
$$

$\begin{aligned} \text { Analyte }= & \text { Vansdium } \\ \text { MPV }= & 974 \mu \mathrm{g} / \mathrm{L} \\ \text { F-pseudosigma }= & 1.04 \\ n= & 26\end{aligned}$

\section{Aluminum $33.6 \mu \mathrm{g} / \mathrm{L}$ \\ 4.5}

39

Calclum

$37.6 \mathrm{mg} / \mathrm{L}$

$0.9(1.68)$

51

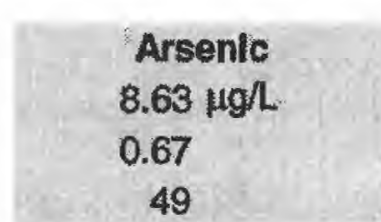
Boron
$24.5 \mu \mathrm{g} / \mathrm{L}$
1.8
21

\section{Cobalt \\ $1.91 \mu \mathrm{g} / \mathrm{L}$ \\ 0.17 \\ 30}

49

Iron

$11.1 \mu \mathrm{g} / \mathrm{L}$

3.6

30

\section{Molybdenum \\ $70.6 \mu \mathrm{g} / \mathrm{L}$ \\ 1.7 (3.5) \\ 39}

\section{Selenium \\ $2.94 \mu \mathrm{g} / \mathrm{L}$ \\ 0.37 \\ 33}

Vanadlum

$7.42 \mu \mathrm{g} / \mathrm{L}$

0.38

33

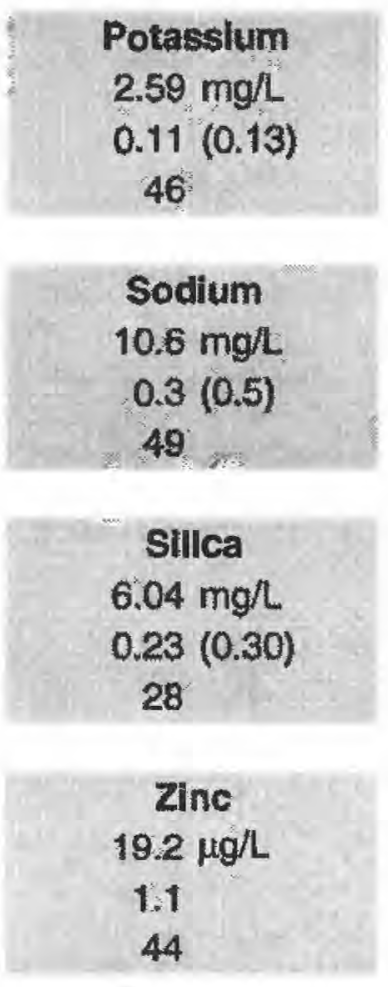

Lithlum

$9.60 \mu \mathrm{g} / \mathrm{L}$

0.61

18

Nickel

$10.3 \mu \mathrm{g} / \mathrm{L}$

0.7

41

\section{Strontlum $174 \mu \mathrm{g} / \mathrm{L}$ \\ 6 (9) \\ 34}

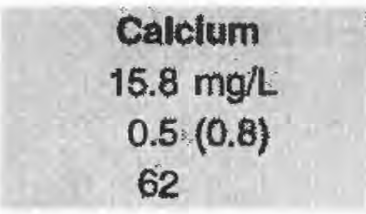

\section{Chlorlde \\ $26.5 \mathrm{mg} / \mathrm{L}$ \\ $1.0(1.3)$ \\ 61}

24

Magnesium
$5.80 \mathrm{mg} / \mathrm{L}$
$0.23(0.29)$
60

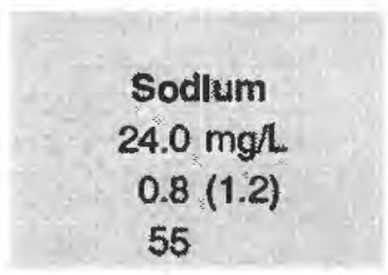

6.60

$0.21(0.33)$

52

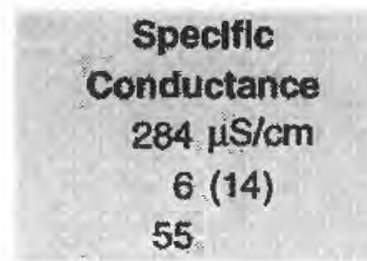

\section{Sulfate \\ $13.4 \mathrm{mg} / \mathrm{L}$ \\ 0.9 \\ 59}
Strontlum $93.8 \mu \mathrm{g} / \mathrm{L}$ $3.2(4.7)$ 31

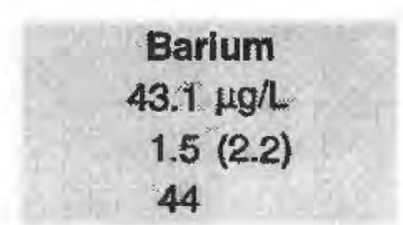

Chromjum

$8.17 \mu \mathrm{g} / \mathrm{L}$.

$0.39(0.41)$

43
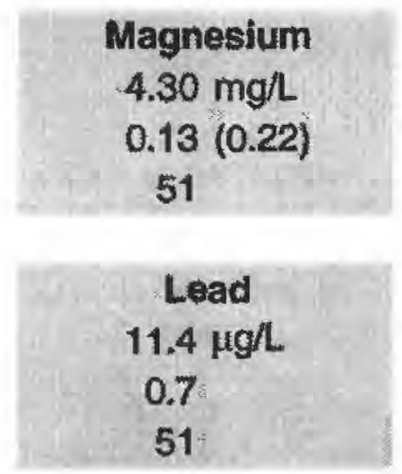

Thallium $4.80 \mu \mathrm{g} / \mathrm{L}$ 0.51 33

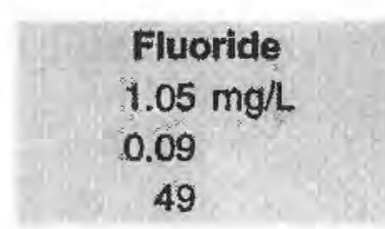

Residue on

Evaporation $188 \mathrm{mg} / \mathrm{L}$

17

38

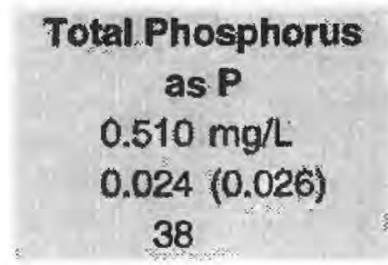


Table 17. Most probable values for constituents and properties in standard reference samples dlstrlbuted in March 2002 -- continued

[MPV, most probable value; $n$, number of samples; $\mu g / L$, microgram per liter; $\mathrm{mg} / \mathrm{L}$, milligram per liter; $\mu \mathrm{S} / \mathrm{cm}$, microsiemens per centimeter at 25 degres Celsius; ( ) rating criterion]

\begin{tabular}{|c|c|c|c|c|c|}
\hline $\begin{array}{r}\text { N-73 } \\
\text { Analyte }= \\
\text { MPV }= \\
\text { F-pseudosigma }= \\
n=\end{array}$ & $\begin{array}{l}\text { Ammonla } \\
\text { as } \mathrm{h} \\
0.127 \mathrm{mgh} \\
0.010 \\
53\end{array}$ & $\begin{array}{l}\text { Ammonia + } \\
\text { Organic N as N } \\
0.140 \mathrm{mg} / \mathrm{L} \\
0.022 \\
36\end{array}$ & $\begin{array}{l}\text { Nituate } \\
\text { as } \mathrm{N} \\
0.126 \mathrm{mg} / \mathrm{L} \\
0.007 \\
54\end{array}$ & $\begin{array}{c}\text { Total Phosphorus } \\
\text { as P } \\
0.132 \mathrm{mg} / \mathrm{L} \\
0.009 \\
51\end{array}$ & $\begin{array}{c}\text { Orthophosphate } \\
\text { as P } \\
0.128 \mathrm{mg} / \\
0.004(0.006) \\
51\end{array}$ \\
\hline $\begin{array}{r}\text { N-74 } \\
\text { Analyte }= \\
\text { MPV }= \\
\text { F-pseudosigma }= \\
n=\end{array}$ & $\begin{array}{l}\text { Ammonla } \\
\text { as } \mathrm{N} \\
0.760 \mathrm{mg} / \mathrm{L} \\
0.037(0.038) \\
49\end{array}$ & $\begin{array}{c}\text { Ammonia + } \\
\text { Organic } \mathrm{N} \text { as } \mathrm{N} \\
0.838 \mathrm{mg} / \mathrm{L} \\
0.113 \\
39\end{array}$ & $\begin{array}{l}\text { Nitrate } \\
\quad \text { as } \mathrm{N} \\
0.711 \mathrm{mg} / \mathrm{L} \\
0.122 \\
58\end{array}$ & $\begin{array}{c}\text { Total Phosphorus } \\
\text { as P } \\
0.755 \mathrm{mgh} \\
0.031(0.038) \\
46\end{array}$ & $\begin{array}{c}\text { Orthophosphate } \\
\text { as P } \\
0.741 \mathrm{mgh} \\
0.085(0.037) \\
49\end{array}$ \\
\hline $\begin{array}{r}\text { P-38 } \\
\text { Analyte }= \\
\text { MPV }= \\
\text { F-pseudosigma }= \\
n=\end{array}$ & $\begin{array}{c}\text { Acldity } \\
\text { inadequate data }\end{array}$ & $\begin{array}{l}\text { Caicium } \\
6.40 \mathrm{mg} / \mathrm{L} \\
0.33 \\
33\end{array}$ & $\begin{array}{l}\text { Chloride } \\
8.37 \mathrm{mg} / \mathrm{L} \\
0.39(0.42) \\
28\end{array}$ & $\begin{array}{c}\text { Fluoride } \\
\text { inadequate data }\end{array}$ & $\begin{array}{c}\text { Potassium } \\
0.830 \mathrm{mg} / \mathrm{L} \\
0.081 \\
32\end{array}$ \\
\hline $\begin{array}{r}\text { Analyte= } \\
\text { MPV }= \\
\text { F-pseudosigma }= \\
n=\end{array}$ & $\begin{array}{c}\text { Magnealum } \\
1.15 \mathrm{mg} / \mathrm{L} \\
0.05(0.06) \\
33\end{array}$ & $\begin{array}{l}\text { Sodium } \\
1.80 \mathrm{mg} / \mathrm{L} \\
0.12 \\
32\end{array}$ & $\begin{array}{l}\text { pH } \\
0.01 \\
0.21(0.30) \\
26\end{array}$ & $\begin{array}{l}\text { Orthophosphate } \\
\text { as P } \\
\text { inadequate data }\end{array}$ & $\begin{array}{l}\text { Sulfate } \\
2.99 \mathrm{mg} / \mathrm{L} \\
0.21 \\
28\end{array}$ \\
\hline $\begin{array}{r}\text { Analyte }= \\
\text { MPV }= \\
\text { F-pseudosigma }= \\
n=\end{array}$ & $\begin{array}{l}\text { Specifle } \\
\text { Conductance } \\
65.0 \mu \mathrm{H} / \mathrm{cm} \\
3.3 \\
29\end{array}$ & & & & \\
\hline
\end{tabular}

HG-34

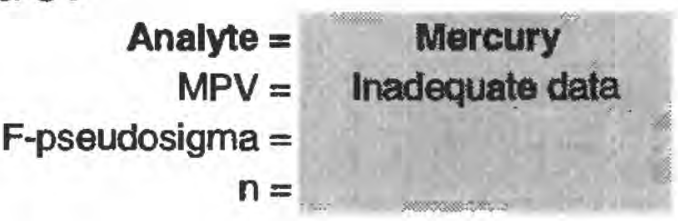

